\title{
THEORY AND DESIGN OF MICROWAVE PHOTONIC FRED-DLECTRON LASERS
}

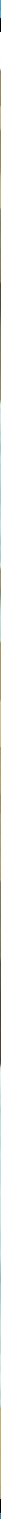





\section{Cover:}

Front: The author's impression of a photonic free-electron laser (pFEL). Multiple electron beams stream through a photonic crystal consisting of an array of rods. Due to the photonic crystal part of the kinetic energy of the electrons is transformed into a coherent electromagnetic wave.

Back: A cross-section through the pFEL construction model for the realization of a microwave pFEL. Electrons are injected on the right side of the picture by an electron gun into a shielded solenoid magnet with a hollow core (red). Inside the magnet a vacuum vessel containing the photonic crystal is placed. The radiation emitted in the photonic crystal propagates down an empty rectangular waveguide towards a vacuum window and can be conveniently characterized outside the vacuum vessel. 


\section{Theory and Design of Microwave Photonic Free-Electron Lasers}

Thomas Denis 
Promotiecommissie:

prof. dr. K.-J. Boller

University of Twente

dr. P. J. M. van der Slot

University of Twente

prof. dr. ir. G. J. M. Krijnen

University of Twente

prof. dr. C. Paoloni

Lancaster University

prof. dr. W. L. Vos

University of Twente

prof. dr. W. J. van der Zande

Radboud University Nijmegen

prof. dr. ir. H. J. W. Zandvliet University of Twente

The research presented in this thesis was carried out at the Laser Physics and Nonlinear Optics Group, MESA+ Institute for Nanotechnology, Department of Science and Technology, University of Twente, P.O. Box 217, 7500 AE Enschede, The Netherlands. The research was also part of the strategic research orientation Applied Nanophotonics within the MESA+ Institute.

This research was financially supported by the Dutch Technology Foundation STW (08128), applied science division of NWO and the Technology Program of the Ministry of Economic Affairs. The author further thanks ESA-ESTEC for providing part of the RF-equipment.

Copyright (C) 2012 Thomas Denis, Enschede, The Netherlands. All rights are reserved.

Printed by Ipskamp PrintPartners, Enschede, The Netherlands

ISBN 978-90-365-3458-1

DOI http://dx.doi.org/10.3990/1.9789036534581 


\title{
Theory and Design of Microwave Photonic Free-Electron Lasers
}

\author{
PROEFSCHRIFT
}

ter verkrijging van

de graad van doctor aan de Universiteit Twente, op gezag van de rector magnificus, prof. dr. H. Brinksma, volgens het besluit van het College voor Promoties in het openbaar te verdedigen op vrijdag 14 december 2012 om 12.45 uur

door

Thomas Denis

geboren op 30 November 1981

te Ahaus, Duitsland 
Dit proefschrift is goedgekeurd door de promotor prof. dr. K. - J. Boller

en de co-promotor dr. P. J. M. van der Slot 
Für meine Eltern, 



\section{Summary}

Coherent electromagnetic waves are extensively used in various fields of research and many applications. Almost every part of the electromagnetic spectrum, ranging from radio waves to hard X-rays, has been put to work for the benefit of mankind. Therefore, it is not surprising that, despite a huge variety of existing sources of electromagnetic waves, there is a continuous demand for novel sources with improved properties tailored to particular needs.

An important, recent development in this ongoing strive for new sources is the use of materials that are periodically structured on the scale of the electromagnetic wavelength, so-called photonic crystals, which fundamentally control the emission of light. This control enables scientists to devise photonic-crystal lasers with unique properties. Examples are ultra-fast modulated lasers or lasers with ultra-low threshold. Due to the employment of small, wavelength sized structures, these lasers are inherently suited for operation in a chip-sized format. For instance, photonic-crystal lasers may very well complement diode lasers in lab-on-a-chip applications that greatly simplify the analysis of biological and chemical samples. To create an even wider applicability of photonic-crystal based lasers, it is crucial to provide many different frequency ranges in a compact format.

While the unique control of periodic structures is frequency scalable, so far the operation of photonic crystal lasers is limited to certain spectral ranges. This is because, to date, photonic-crystal lasers are based on certain discrete bound-electron transitions, typically provided by specific semiconductors or quantum dots. On the other hand, the emission from free electrons is not subject to such limitation. When free electrons undergo transitions between the continuously distributed states of kinetic energy they can, in principle, emit any frequency. It is thus very desirable to combine the capability of free electrons to generate electromagnetic waves over huge frequency ranges with the frequency scalable control offered by photonic crystals, to generate coherent light.

In this thesis we study this novel type of photonic-crystal laser that is based on coherent emission from free electrons. We name this laser a photonic free-electron laser (pFEL). 
We present in this thesis the first comprehensive study of the properties of a pFEL. As pre-condition we limit the scope of this thesis to pFELs that use low energy electron beams (about $10 \mathrm{keV}$ ). These electron energies can be produced by compact electron sources to achieve compact devices. The thesis presents also first steps for an experimental realization of pFELs at microwave frequencies.

For studying the working principle and laser properties of the pFEL we first numerically investigate an example of a laser driven by a single electron beam using a particlein-cell (PIC) method. This pFEL is pumped by an electron beam with a beam energy of about $12.5 \mathrm{keV}$ and a beam current of around $1 \mathrm{~A}$. The photonic crystal has millimeter dimensions and is set up from a periodic array of metallic rods placed inside a rectangular metallic waveguide, which should yield operation at microwave frequencies. For this investigated example of a pFEL we find a current threshold of about $140 \mathrm{~mA}$. Increasing the pump current to a value of $1 \mathrm{~A}$ leads to a linear scaling of the output power to values in the order of $1.5 \mathrm{~kW}$ at an output frequency near $16 \mathrm{GHz}$.

The PIC calculations for this specific example of a pFEL further show that the emission of a pFEL can be well described within the framework of a simple working principle. This working principle is based on the constructive interference of multiple wavelets emitted from the photonic crystal as response to the passing electrons. The emission of each of these wavelets can be viewed as spontaneous emission of Čerenkov radiation from single electrons inside the photonic crystal. The Čerenkov emission process becomes stimulated if the photonic crystal control provides a suitable electromagnetic mode. This mode has to possess a longitudinal electric field component and a phase velocity component which matches the velocity of the electrons, such that the electromagnetic wave can form electron bunches from the initially continuous stream of electrons. During the formation of electron bunches on average more electrons are decelerated than accelerated and the associated net reduction of kinetic energy in the electron beam is converted into a growing electromagnetic wave. Via tuning the electron velocity, the velocity-matched frequency is changed which allows frequency tuning of the laser output.

Using PIC methods gives a detailed insight into the pFEL operation mechanism and performance, however, such detailed description is not always necessary. Especially, as PIC modeling suffers from long calculation times, an alternative approach to assess the suitability of photonic crystals for use in a pFEL is desirable. This advantage is crucial when the suitability of different kinds of photonic crystals has to be quickly compared, and when a wide range of pump parameters has to be explored, such as for preparing an experimental demonstration. We address this inherent problem of PIC calculations by verifying for the first time for a pFEL that a linearized gain model can compute the laser 
threshold and small-signal gain rather well.

While the high power of $1.5 \mathrm{~kW}$ generated by a single-beam pFEL might be promising for a variety of microwave applications, an even higher promise lies in the fundamental frequency scalability of pFELs. When reducing the spatial period of the crystal by a chosen factor to increase the laser frequency of a pFEL with the same factor, the output power would only remain constant if the other laser parameters are kept constant. The latter means particularly the energy and the current of the single electron beam that pumps the laser. The problem with this is the following. When reducing the crystal period also the cross-sectional area available for electron beam propagation reduces and the pump current can only be kept constant when increasing the current density, accordingly. Current densities of electron beams are, however, fundamentally limited to certain maximum values which are given by the Coulomb repulsion of the electrons. Thus, upon frequency up-scaling the current in the single electron beam will inevitably reduce and, consequently, also the generated output power.

Fortunately, photonic crystals offer an approach for achieving frequency scaling while maintaining a constant output power. Photonic crystals naturally provide many vacuum channels in parallel. Thus, by adding more electron beams, such that the total beam current remains constant upon frequency scaling, the output power can remain constant. Such scaling would, however, require that adding more pump beams to a pFEL not only increases output power, but that no transverse mode oscillation is introduced which would decrease the brightness of the output.

For investigating this latter, central property, we increase the number of transverse photonic crystal periods such that the laser can be driven by up to seven electron beams. The calculated output power is observed to increase with the number of pump beams and reaches about $8 \mathrm{~kW}$ with seven beams. Importantly, the laser remains oscillating in a single mode, i.e., the lowest transverse mode remains dominating and contains more than $95 \%$ of the total output power. This single-mode power scaling is most likely caused by the fact that the electron beams are mono-energetic which leads to a gain competition between the transverse modes that is similar to homogeneous gain broadening of spectrally different modes in solid-state lasers.

Using this power scaling, we envision that in the future the total pump current for a pFEL might eventually be distributed over hundreds or thousands of low current pump beams. Such arrays of electron beams may be provided, e.g., by field-emitter arrays. The electron beams of these arrays should pump a single-mode of a pFEL at THz frequencies and generate several watts of output power with a high spectral and spatial brightness.

The second part of the thesis is aimed to prepare the realization of a microwave pFEL. 
The key element to provide amplification in a pFEL is the photonic crystal. More specifically, the crystal has to provide a longitudinal electric field component at the position of the electron beam. This field component determines the absolute strength of amplification. It is thus important to experimentally characterize this field component for photonic crystals considered for pFELs. However, to date, measurements of individual field components well inside a photonic crystal have never been undertaken.

For mapping the absolute value of individual electromagnetic field components inside photonic crystals we present and demonstrate such a measurement technique. The method relies on measuring the change in resonance frequency when the photonic crystal is placed inside a resonator and the field inside the photonic crystal is perturbed by a subwavelength scatterer. In our experiments a spherical scatterer is applied to measure the dominating longitudinal electric field in a specific photonic crystal slab. We observe good agreement between measured and calculated longitudinal electric field strength without using any adjustable parameters in the calculations.

Our numerical investigations clearly indicate that the concept of pFELs is highly promising for generating frequency tunable coherent Čerenkov radiation of appreciable output power. To prepare also an experimental study of such lasers, we present a design for realizing a pFEL at microwave frequencies. This requires to combine four different technologies, i.e., electron beam generation and transport, high-voltage technology, microwave engineering and vacuum technology. A commercial dispenser electron gun, usually applied in traveling-wave tubes, is selected as electron source. This particular electron gun provides about $2 \mathrm{~A}$ of beam current at its nominal beam energy of $14.2 \mathrm{keV}$. For studying the frequency tuning of a pFEL the beam energy can be varied in a range of $10 \mathrm{keV}$ to $15 \mathrm{keV}$. The required high-voltage power supply to operate the gun is presented and realized. Further, we present the design and realization of an electromagnet for guiding the electron beam through a photonic crystal and we investigate the associated electron flow. We present the design and fabrication of a photonic crystal. The experimental realization allows to use a photonic crystals that comprises more than one hundred unit cells. In this case we expect a low pump current of about $7 \mathrm{~mA}$ necessary for reaching the laser oscillation threshold. We expect that, after fully assembling the setup, the first systematic experimental analysis of a pFEL becomes possible.

The theoretical modeling presented in this thesis and the subsequent experimental demonstration and laser operation based on this thesis may become the key for the emergence of a new family of compact and high-power laser sources for the microwave to $\mathrm{THz}$ spectral range. 


\section{Contents}

Summary $\quad$ i

1 Introduction 1

2 Theoretical foundations 5

2.1 Introduction . . . . . . . . . . . . . . . . . . . 5

2.2 The Cerenkov effect . . . . . . . . . . . . . . . . . . 8

2.3 Basic equations . . . . . . . . . . . . . . . . . . . . . . 10

2.4 Photonic crystals . . . . . . . . . . . . . . . . . . . . 12

2.5 Electromagnetic waves on electron beams . . . . . . . . . . . . 18

2.6 Linear theory of slow-wave free-electron lasers . . . . . . . . . . . . . . . . 22

2.7 Nonlinear theory: particle-in-cell calculations . . . . . . . . . . . . . . . 28

3 Photonic free-electron laser pumped by a single electron beam 33

3.1 Working principle of a pFEL . . . . . . . . . . . . . . . . . 36

3.2 pFEL model . . . . . . . . . . . . . . . . . . . . . . . . . . . . . . 39

3.3 Particle-in-cell calculations . . . . . . . . . . . . . . . . . . 44

3.3.1 Laser operation with fixed electron velocity . . . . . . . . . . . . 44

3.3.2 Tuning of electron velocity . . . . . . . . . . . . . 55

3.4 Comparison of particle-in-cell calculations to linearized theory . . . . . . . 58

3.5 Conclusion and outlook . . . . . . . . . . . . . . . . . . . 61

4 Photonic free-electron laser pumped by multiple electron beams $\quad 63$

4.1 Introduction . . . . . . . . . . . . . . . . . . . . . . . 63

4.2 pFEL model and dispersion . . . . . . . . . . . . . . . . 66

4.3 PIC calculations . . . . . . . . . . . . . . . . . . . . . . . . . . 69

4.4 Mode competition . . . . . . . . . . . . . . . . . 71

4.5 Power scaling for multiple beams . . . . . . . . . . . . . . . . . 77 
4.6 Conclusion . . . . . . . . . . . . . . . . . . . . . . . . . 82

5 Mapping electromagnetic field components inside photonic crystals $\quad 85$

5.1 Introduction . . . . . . . . . . . . . . . . . . . 85

5.2 Measurement method . . . . . . . . . . . . . . . . 87

5.3 The photonic crystal slab . . . . . . . . . . . . . . . . . . 88

5.4 Experimental setup . . . . . . . . . . . . . . . . . . 91

5.5 Dispersion measurement . . . . . . . . . . . . . . . . . . 93

5.6 Electric field measurements . . . . . . . . . . . . . . . . . . . 95

5.7 Conlcusion and outlook . . . . . . . . . . . . . . . . . 98

6 Design of a photonic free-electron laser 101

6.1 Introduction . . . . . . . . . . . . . . . . . . . . . . . 101

6.2 Setup overview . . . . . . . . . . . . . . . . . . . . 102

6.3 Electron generation . . . . . . . . . . . . . . . . . . 105

6.4 Electron beam transport . . . . . . . . . . . . . . . . . . . 108

6.5 Microwave design . . . . . . . . . . . . . . . . . . . . 114

6.6 Conclusion and outlook . . . . . . . . . . . . . . . . . . 117

7 Conclusions and outlook $\quad 119$

$\begin{array}{ll}\text { A Laser resonators for pFELs } & 127\end{array}$

A.1 Transmission at the interface . . . . . . . . . . . . . . . . . . . 129

A.2 Reflection at the interface . . . . . . . . . . . . . . 134

$\begin{array}{ll}\text { Bibliography } & 137\end{array}$

$\begin{array}{ll}\text { Samenvatting } & 161\end{array}$

$\begin{array}{lr}\text { List of publications } & 167\end{array}$

$\begin{array}{ll}\text { Acknowledgment } & 173\end{array}$ 


\section{1}

Introduction

Galileo Galilei, the father of modern physics [1], was one of the first to actively control light by using materials. In his time he used curved glass surfaces to guide light to form a telescope for observing the moons of Jupiter [2]. Nowadays, controlling the flow of light through optical fibers is one of the foundations of the internet [3]. Within the blink of an eye, text, music or video clips can be sent around the globe, which has changed society in a most dramatic way [4]. This single example demonstrates the importance of light, or more generally of electromagnetic waves, in society. Almost every part of the electromagnetic spectrum, ranging from radio waves to hard X-rays, has been put to work for the benefit of mankind [3,5-15]. Therefore, it is not surprising that, despite a huge variety of existing sources of electromagnetic waves, there is a continuous demand for novel sources with improved properties tailored to particular needs.

A recent development in this ongoing strive for new sources is, for example, the use of materials that are periodically structured on the scale of the electromagnetic wavelength, so-called photonic crystals, which fundamentally control the emission of light $[16,17]$. The emission generated within photonic crystals is temporally and spatially shaped due to a modification of the local radiative density of the electromagnetic states [18]. While initially only theoretically predicted, recently, a number of periodic materials, such as metamaterials, plasmonic structures and photonic crystals have experimentally demon- 
strated the ability to radically alter the emission of embedded light sources, such as quantum dots, molecules and ions [19-29].

This control enables scientists to devise photonic crystal lasers with unique properties. Examples are ultra-fast modulated lasers or lasers with ultra-low threshold [30-34]. Due to the employment of small, wavelength sized structures, these lasers are inherently suited for operation in a chip-sized format [35-38]. For instance, photonic-crystal lasers may very well complement diode lasers in, for example, lab-on-a-chip applications that greatly simplify the analysis of biological and chemical samples [39-43]. To create an even wider applicability of photonic-crystal based lasers, it is crucial to provide many different frequency ranges in a compact format. Fortunately, the unique control of periodic structures is frequency scalable, which is a direct consequence of the scale invariance of Maxwell's equations [44]. This means that a certain photonic crystal geometry provides exactly the same electromagnetic properties and control, when appropriately scaled into a different frequency range. Despite this unique potential for scaling, photonic crystal lasers are only available in very selected frequency ranges so far [32]. This is because, to date, photonic-crystal lasers are based on bound-electron transitions, typically within semiconductors $[31,32]$ or quantum dots $[30,33]$. The discreteness of energy levels of bound electrons fundamentally limits the emission to a restricted range of the electromagnetic spectrum.

The emission from free electrons is not subject to such limitation. Free electrons can emit over huge spectral ranges, when undergoing transitions between states of different kinetic energy [45]. As the distribution of these states is continuous, any wavelength can a priori be generated. Free-electron lasers (FELs) operate at the extremes of the electromagnetic spectrum as well as any region in between, i.e., from microwaves all the way up to hard X-rays $[46,47]$. The generation of coherent electromagnetic waves by stimulated emission in FELs is based on providing a close synchronism between the initial velocity of the electrons and the velocity of the electromagnetic wave to be generated, such that a longitudinal force allows to alter the kinetic energy of the electrons in the electron beam [48]. The frequency of the electromagnetic wave required for this velocity-matching changes upon variation of the electron velocity. Hence, FELs are continuously tunable via the velocity of the injected electrons. It is thus very desirable to combine the capability of free electrons to generate electromagnetic waves over huge frequency ranges with the frequency scalable control offered by photonic crystals, to generate coherent light - a free-electron laser based on photonic crystals.

The typical approach to the generation of coherent light with FELs is what is called a fast-wave FEL. There, velocity-matching is achieved via using an alternating static 
magnetic field of a so-called undulator. The presence of the undulator field allows converting the kinetic energy of relativistic electrons into coherent electromagnetic waves. These waves travel at the high vacuum velocity of light, $c$ [49]. While this concept can be applied in entirely different spectral ranges, for example to provide $\mathrm{THz}$ or X-ray laser radiation [50-53], the major drawback is that velocity-matching requires highly relativistic electron energies in the $\mathrm{MeV}$ to $\mathrm{GeV}$ range. Providing electrons with such energies is only possible via large-scale accelerators surrounded by a radiation shield and fed by bulky klystrons [53]. Indeed, these lasers are typically run only as exclusive user facilities that provide unique research capabilities, but which also exclude wide-spread applications.

The alternative approach to achieve velocity-matching is by slowing down the electromagnetic wave via so-called slow-wave structures. Hence, devices based on this principle are called slow-wave FELs and include Čerenkov FELs, Smith-Purcell FELs, travelingwave tubes and backward-wave oscillators [8, 54-61]. Notably, the slow-wave structures used in traveling-wave tubes and backward-wave oscillators are well-suited for a significant reduction of the velocity of the electromagnetic wave. A small fraction of the speed of light, such as $0.15 c$ is easily reached [62]. This allows velocity-matching with low-energy electron beams having kinetic energies in the order of $10 \mathrm{keV}$. Thereby, very compact designs for the electron source can be used $[63,64]$. Unfortunately, the geometry of standard slow-wave structures in traveling-wave tubes and backward-wave oscillators, such as a metal helix inside a waveguide, limit the operating frequency to the microwave range. This is because typical slow-wave structures allow the injection and pumping with only a single electron beam [65]. This implies that, upon frequency up-scaling, the maximum current driving the device inevitably reduces. The reason is that Coulomb repulsion between the electrons, even when using external focusing, renders it effectively impossible to send an electron beam through slow-wave structures beyond a certain electron density $[64,66]$. As the feature size and periodicity of the slow-wave structure is set by the desired operating frequency, the current driving the device decreases when increasing the frequency. The current is proportional to the gain of the FEL and this means that a sharp decrease in performance is found when attempting to scale the frequency up from the microwave range to $\mathrm{THz}$ frequencies $[64,65,67]$.

Due to their spatial periodicity photonic crystals can essentially be seen as a slowwave structure as well. Correspondingly, a large reduction in the propagation velocity of electromagnetic waves inside the crystal has also been demonstrated [62]. However, in contrast to standard slow-wave structures, photonic crystals can also be periodic in the transverse direction [29]. Thereby, photonic crystals can provide many channels for electron beam propagation in parallel. When down-scaling the spatial period of a photonic 
crystal for up-scaling the frequency, the absolute size of the channels decreases as well. As a result also the current that can be transported through an individual channel inevitably drops. However, utilizing the transverse extent of the photonic crystal, many electron beams in parallel can be sent through the crystal [68]. In this way, the current density in each beam can be held sufficiently low to maintain the transport of the beam feasible, while the total current can then be increased via the number of beams. This way the total beam current through the photonic crystal can be easily made far larger than the maximum current in other slow-wave FELs. Hence, utilizing photonic crystals as the gain medium for a FEL should enable to provide an increased gain and thereby also a more powerful laser output [69].

In this thesis we study a novel type of photonic-crystal laser based on coherent emission from free electrons, which we call the photonic free-electron laser (pFEL). For convenience we investigate the basic properties of pFELs at microwave frequencies, where photonic crystal fabrication technology and electron beam technology is readily available. This thesis describes a numerical modeling of such pFELs, and presents first steps towards an experimental realization. Chapter 2 introduces the key concepts for obtaining stimulated emission from free electrons inside photonic crystals. These concepts are then applied in chapter 3 to study the generation of coherent electromagnetic waves from a single electron beam streaming through a photonic crystal. While such single-beam pFEL shows high promise for generating high-power microwaves, chapter 4 presents an extension towards many electron beams as a route towards a frequency up-scaling without power loss. Chapter 5 presents a experimental method to characterize the spatial distribution of individual electromagnetic field components inside photonic crystals, such as for investigating the suitability of a photonic crystal for use in a pFEL. Chapter 6 presents the overall design of a single-beam pumped pFEL, which we expect to enable the demonstration and characterization of a pFEL in the near future. Finally, conclusions and suggestions for future work are presented in chapter 7 . effect of a shrinking channel diameter and current per beam. 


\section{2 \\ Theoretical foundations}

\section{$2.1 \quad$ Introduction}

The photonic free-electron laser ( $\mathrm{pFEL}$ ) may be viewed as a particular example of a freeelectron laser (FEL), as termed originally by John Madey in 1971 [49]. Madey described the FEL in his paper with a quantum-mechanical formalism. It was discovered later that FELs can be very accurately described by classical mechanics and electrodynamics. Since then, many different formalisms have been presented which self-consistently describe electromagnetic field generation and particle propagation in FELs. These include Hamiltonian treatments [70], descriptions using the Maxwell-Vlasov distribution function [71], and direct solution of Maxwell's and the Newton-Lorentz equations [46, 47]. A unified theory was published by Gover [48], showing that any FEL fundamentally satisfies the same gain-dispersion relation and can be described by a single coupling parameter. Most importantly, Gover pointed out that a close matching between the propagation velocities of an electromagnetic wave and the electrons is a necessary condition for the generation of coherent radiation in any FEL. If such, so-called, velocity-matching is achieved by decelerating the phase velocity of the electromagnetic wave, the FEL is classified as a slow-wave FEL, otherwise the laser is classified as a fast-wave FEL.

In a pFEL a photonic crystal is used to slow down the phase velocity of the wave 
to achieve velocity-matching with the electron beam. Therefore, we can classify a pFEL as a slow-wave FEL. As such the theoretical foundations of pFELs should be closely related to other types of slow-wave FELs, specifically, the Čerenkov FEL [58, 59, 72-79] or the Smith-Purcell FEL [57, 60, 61, 80-85]. Also, certain types of microwave tubes, including the traveling-wave tube, backward-wave oscillator or orotron, rely on the same physical principles as slow-wave FELs [8, 54-56, 86-102]. Despite this, microwave tubes are usually distinguished from FELs for historical reasons. For convenience of writing and clarity, however, we will not follow such further distinctions in this thesis.

The most important difference amongst all existing slow-wave FELs is the type of slowwave structure that decelerates the wave propagation. To slow down the electromagnetic wave pFELs use photonic crystals and Čerenkov FELs use dielectric lined waveguides. Smith-Purcell FELs use gratings and microwave tubes electrical circuits. The electromagnetic field distributions in this variety of slow-wave structures are completely different and one might think that this strongly changes the principles of operation. However, it turns out that the basic principles of all slow-wave FELs can be described by referring to the Čerenkov effect [103]. The origin of spontaneous Čerenkov emission is an interference of randomly phased Čerenkov emission events from single electrons. In order to obtain stimulated emission, electron bunching needs to be present on the electron beam. Stimulated Čerenkov emission can then be interpreted as a well-phased interference of Čerenkov emission events from single electrons. As the emission of single electrons in periodic structures has been shown to be an example of the well-known Čerenkov effect in bulk, homogeneous dielectric media [104], the general working principle of slow-wave FELs can be understood by considering the emission of single electrons in an effective dielectric with a suitable refractive index. However, a more thorough analysis of slow-wave FELs requires to self-consistently solve the basic equations that include the detailed spatial structure of the electromagnetic field distribution and the spatial and kinetic distribution of the electrons.

In a classical framework, Maxwell's equations coupled to the Newton-Lorentz equation describe the emission of slow-wave FELs. However, these nonlinear differential equations are very complex and hard to solve, even in strongly simplified situations [105]. To enable an analytic solution, the standard approach is to first assume that the laser amplifies radiation of only a single, specific eigenmode of the slow-wave structures. Second, one verifies to what extent one can neglect the influence of Coulomb forces between electrons of the beam, which excite so-called space-charge waves. While such analysis has been given for some specific slow-wave FELs [91, 106, 107], it has never been given for a pFEL, probably due to the lack of analytic solutions for the modes of photonic crystals. 
By linearizing the Maxwell and Newton-Lorentz equations, the system of equations can be solved in a largely simplified and general form [54, 103]. For certain slow-wave structures the small-signal gain can then be computed from numerically calculated eigenmode solutions. For theses specific structures, such calculations can further estimate the pump current at which oscillations set in (threshold current), the oscillation frequency, and the gain bandwidth of the laser. Due to the relative simplicity of these linear theories, it is very important to investigate whether this description is indeed valid when applied to photonic crystals. Such validation requires a comparison to a fully nonlinear description. More importantly, what a linear theory cannot well predict is the steady-state output power or the way several simultaneously excited modes compete for the available gain in the laser. The reason is that these properties are the result of nonlinear effects dominating the laser dynamics.

To predict these properties the only alternative is a direct "brute-force" solution, for example provided by particle-in-cell (PIC) methods [108-110]. While in the past the applicability of such methods has been strongly limited by the lack of affordable computational power, the ever increasing performance of desktop PCs now enables such computations on single workstations. A tremendous benefit of PIC methods is that complex systems with strongly nonlinear dynamics can be investigated with using very little approximate assumptions. Essentially, all physical effects described by the differential equations are inherently included in the numerical solutions. The solution can even include subtle nonlinear effects, such as the modification of the eigenmodes of the photonic crystal caused by the presence of the electron beam.

To summarize, the theoretical description of a pFEL fundamentally relies on the Cerenkov effect, which we discuss in section 2.2. For a quantitative treatment of the laser operation, solutions for the coupled Maxwell and Newton-Lorentz equations have to be found. These equations are given in section 2.3. To prepare the calculation of the small-signal gain, the properties of the photonic crystal eigenmodes and of the electron beam are given in section 2.4 and 2.5, respectively. The actual derivation of the small-signal gain is given in section 2.6. We conclude the chapter with a brief overview of the approach of PIC methods, which can be used for finding specific and almost nonapproximate solutions of the Maxwell and Newton-Lorentz equations that describe the dynamics of the laser from start-up to steady-state operation. 


\subsection{The Čerenkov effect}

During the 1930s, P. A. Cerenkov performed extensive experimental studies to reveal the origin of the incoherent light emitted from liquids when exposed to gamma radiation [111]. He concluded that the observed faint, blueish emission is caused by the polarizing effect of fast electrons ejected from atoms and molecules of the liquid when hit by the gamma radiation. Frank and Tamm recognized that, to theoretically describe this effect, the ejected electrons must move faster inside the medium than the generated light [112]. Based on this understanding they formulated a theory which is in excellent agreement with the experimental results of Čerenkov. Nowadays, the radiation is called Čerenkov radiation, after its discoverer. An extensive review of the original work was written by Jelly and here we follow his description [113].

Čerenkov radiation is the electromagnetic response caused by a medium through which charged particles are passing. For example, when an electron travels through a medium such as water, it transiently polarizes atoms along its trajectory through the medium. As a response, the polarized atoms or molecules radiate like elementary dipoles causing the emission of a wavelet, i.e., each atom or molecule emits a short pulse of radiation with wide spectral distribution. Two separate cases can be distinguished regarding the electron velocity, $v$, with respect to the phase velocity of the electromagnetic field inside the medium, $v_{p h}$. If the velocity of the electrons is lower than the phase velocity, no propagating field is observed at large distances, as the emission from all dipoles destructively interferes. However, when the electrons overcome the phase velocity of the electromagnetic field inside the medium, net radiation is generated by the locally induced dipole moments. This Čerenkov radiation is observed under a certain observation angle $\theta$ with respect to the propagation axis of the electrons.

The observation angle $\theta$ of Čerenkov radiation can be obtained using a simple geometrical Huygens' construction. Figure 2.1 depicts schematically a single electron propagating inside a dielectric material with a refractive index $n$. The electron travels from point $A$ to point $E$ in a time $\Delta \tau$ with the velocity $v$. For example, suppose water molecules are located at points $A$ to $E$. Each time the electron passes along a molecule, the electron induces the emission of a wavelet from that molecule. This wavelet propagates with the same phase velocity, $v_{p h}$, in all directions, thereby forming a spherical wave. However, recall that all the emitted spherical waves propagate with a phase velocity $v_{p h}$ lower than the electron velocity $v$. As a result, when drawing the wavelets emitted from each molecule at the moment when the electron reaches $E$, we observe that, due to constructive interference, a wave front is formed from $E$ to $F$. To determine the angle $\theta$, which the wave front 

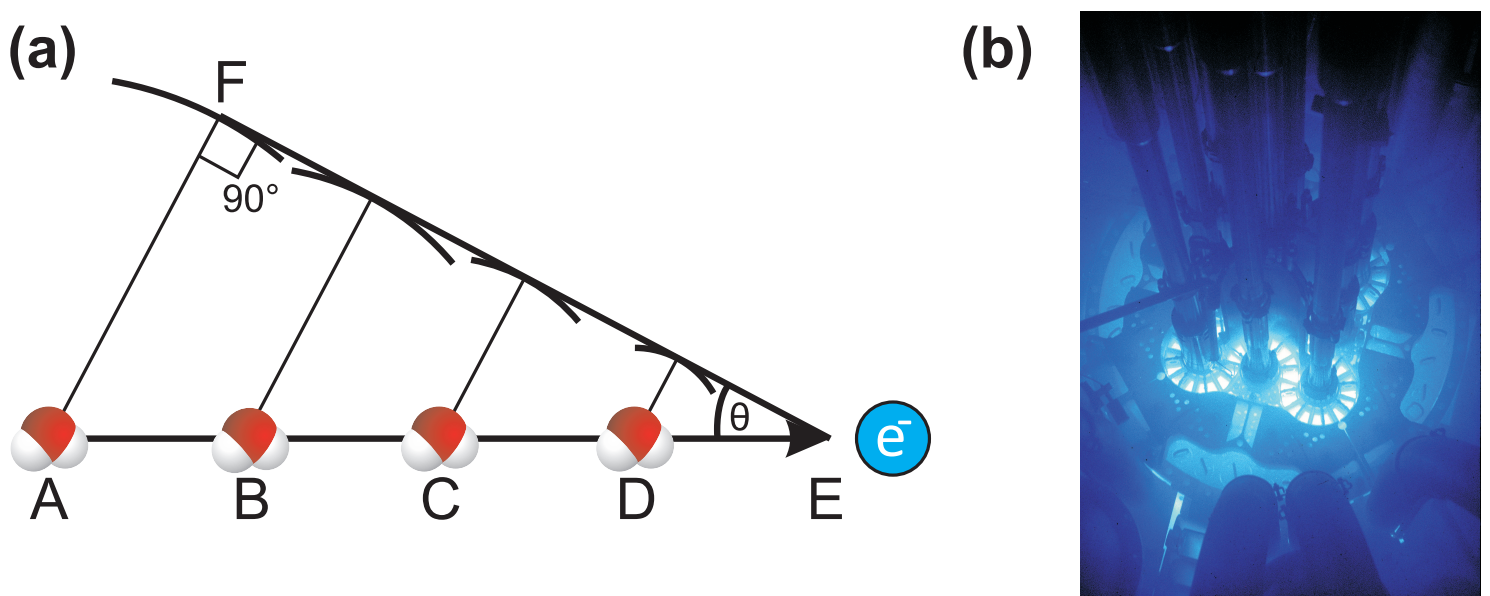

Figure 2.1: (a) Huygens' construction to explain the emission angle and velocity threshold of Čerenkov radiation in a dielectric. (b) Faint blueish glow in a nuclear fission reactor due to Čerenkov emission from high energy charged particles in water (Reproduced with kind permission from Idaho National Laboratory, USA, www.inl.gov).

encloses with the propagation direction of the electrons we analyze the triangle $\triangle A E F$ shown in Fig. 2.1a.

When the electron travels from $A$ to $E$ in a time interval $\Delta \tau$, then the length of the triangle's hypothenuse is $v / \Delta \tau$. During the same interval the wave that has been generated at $A$ is traveling the distance $A$ to $F$, and so the length of the triangle's leg is $v_{p h} / \Delta \tau$. The angle $\theta$ is then

$$
\sin \theta=\frac{v_{p h}}{v} .
$$

This equation only has a real solution when the electron velocity exceeds the phase velocity of the light, which mathematically expresses the existence of a velocity threshold for Čerenkov radiation in a dielectric medium.

As the refractive index $n$ is defined as $c / v_{p h}$, all media in which the refractive index exceeds unity enable the generation of Čerenkov emission. In gases the refractive index is very close to unity, thus requiring electron velocities that are very close to the speed of light $(v>0.99 c)$ [114]. In media of higher density, e.g., solid-state matter and liquids, Čerenkov radiation occurs at somewhat lower electron velocity, but is intermixed with a variety of other effects undesired for slow-wave FELs. For example, Bremsstrahlung is generated and Čerenkov radiation dominates only at high, relativistic electron velocities. A common example of this is the blueish glow of the cooling water surrounding the core of a nuclear fission reactor (Fig. 2.1b).

It is possible to generate Čerenkov radiation without simultaneously generating Bremsstrahlung if the electrons propagate in close proximity to the surface of the medium in- 
stead of being sent directly through the medium. In this case the space-charge field of the electrons still penetrates the medium causing Čerenkov radiation. However, because the space-charge field decreases inversely proportional to the distance between the electron and the surface of the medium, the emission also decreases with the distance to the surface [115]. Further, the emission turns out to be strong only at high electron velocities [116].

From what is said above it appears as if the efficient generation of Čerenkov radiation would always require electron velocities close to the speed of light. This would be extremely undesired for devising compact slow-wave FELs. However, it turns out that Čerenkov radiation can also efficiently be excited with rather low electron velocities at a small fraction of $c$ in so-called effective dielectric media. Examples are electrical circuits, metallic gratings or photonic crystals $[8,117,118]$. In such an effective dielectric medium, both the macroscopic spatial configuration and the refractive indices of the materials define the effective refractive index and phase velocity. Based on these combined effects it has recently been predicted that spontaneous Čerenkov emission of single electrons in a photonic crystal should show no velocity threshold [119]. Furthermore, more complex emission patterns, instead of simple cone shaped emission patterns are expected for these crystals. The reason is that the emitted wavelets propagate in different directions with different velocities, unlike in homogeneous, isotropic dielectrics.

For the sake of completeness let us note that in a photonic crystal Čerenkov radiation is always accompanied by another electron emission process named transition radiation $[119,120]$. In homogeneous media of larger volume (bulk media) both can be strictly distinguished. Čerenkov radiation is generated inside the homogeneous medium, while transition radiation is emitted from the surface when electrons cross a dielectric boundary between two homogeneous media. However, as Luo points out [119], in a photonic crystal both occur simultaneously and a strict distinction between the contributing processes is not possible. For the sake of brevity, in the following we will not further distinguish between these emission processes and use a single term, Čerenkov radiation.

\subsection{Basic equations}

Considering photonic crystal as an effective dielectric medium immediately explains why a single electron emits Čerenkov radiation when streaming through photonic crystals. However, this does not take into account the actual spatial distribution of the local electromagnetic field inside a photonic crystal. The electromagnetic field inside a photonic crystals is clearly different from the field in a homogeneous dielectric [44]. The enhancement and suppression of spontaneous emission from quantum dots inside photonic crystals 
provides a striking example for this [19]. More specifically for our study, the interference of Čerenkov emission from multiple electrons, as used in slow-wave FELs, results in incoherent radiation when no special measures are taken. To describe the generation of coherent Cerenkov emission from multiple electrons the feedback of the electromagnetic field on the beam of free-electrons must be included self-consistently. To consider the actual electromagnetic field distribution inside photonic crystals and the feedback mechanism, a complete theoretical analysis of the pFEL is required.

Electromagnetic fields are described by Maxwell's equations

$$
\begin{aligned}
\nabla \times \mathbf{H}(\mathbf{r}, t) & =\mathbf{J}(\mathbf{r}, t)+\frac{\partial \mathbf{D}(\mathbf{r}, t)}{\partial t} \\
\nabla \times \mathbf{E}(\mathbf{r}, t) & =-\frac{\partial \mathbf{B}(\mathbf{r}, t)}{\partial t} \\
\nabla \cdot \mathbf{B}(\mathbf{r}, t) & =0 \\
\nabla \cdot \mathbf{D}(\mathbf{r}, t) & =\rho(\mathbf{r}, t) .
\end{aligned}
$$

Here $\rho(\mathbf{r}, t)$ is the density of free charges, $\mathbf{J}(\mathbf{r}, t)$ the density of free currents, $\mathbf{E}(\mathbf{r}, t)$ the electric and $\mathbf{H}(\mathbf{r}, t)$ the magnetic field. All quantities can explicitly depend on the location $\mathbf{r}$ and the time $t$. Throughout this thesis the electric displacement field $\mathbf{D}(\mathbf{r}, t)$ is assumed to be linearly dependent on the electric field

$$
\mathbf{D}(\mathbf{r}, t)=\epsilon_{0} \epsilon(\mathbf{r}) \mathbf{E}(\mathbf{r}, t)
$$

where $\epsilon_{0}$ is the vacuum permittivity and $\epsilon$ is the relative permittivity describing the dielectric material properties. This approximation is justified unless the field becomes comparable to the internal binding fields of the dielectric. Note that $\epsilon$ can explicitly depend on the location $\mathbf{r}$ to define the spatial structure of the photonic crystal. For simplicity, we assume here that $\epsilon$ is a scalar. For the magnetic flux density, $\mathbf{B}(\mathbf{r}, t)$, we find

$$
\mathbf{B}(\mathbf{r}, t)=\mu_{0} \mu(\mathbf{r}) \mathbf{H}(\mathbf{r}, t)
$$

where $\mu_{0}$ is the vacuum permeability and $\mu$ is the relative permeability describing the magnetic material properties. In this thesis we discuss only non-magnetic photonic crystals and set $\mu$ to unity.

Feedback on the electron motion due to electric and magnetic fields is described by 
the relativistic Newton-Lorentz equation,

$$
\mathbf{F}=m_{e l} \frac{\mathrm{d} \gamma \mathbf{v}(\mathbf{r}, t)}{\mathrm{d} t}=q_{e l}[\mathbf{E}(\mathbf{r}, t)+\mathbf{v}(\mathbf{r}, t) \times \mathbf{B}(\mathbf{r}, t)]
$$

Here, $\mathbf{F}$ is the force, $q_{e l}=-e$ is the electron charge, $m_{e l}$ the electron mass, $\mathbf{v}$ the electron velocity, and $\gamma$ is the Lorentz factor, which is defined as

$$
\gamma=\frac{1}{\sqrt{1-|\mathbf{v}|^{2} / c^{2}}} .
$$

Via self-consistently solving the set of nonlinear differential equations (2.2)-(2.8), using proper initial and boundary conditions, one would obtain a full description of the generation of coherent Čerenkov emission inside photonic crystals. However, solving this coupled, nonlinear system of differential equations is mathematically very complex, especially, due to the complex boundary conditions needed to describe photonic crystals. A first insight might be possible by linearizing the equations, for example by using the method of Pierce $[54,89]$. The linearization allows the approximate value of the smallsignal gain of certain slow-wave FELs to be determined. This is achieved by modeling the slow-wave structure as effective dielectric that couples electromagnetic waves to the spacecharge waves propagating along the electron beam. In order to prepare the application of this theory also for photonic crystals we first recall the properties of modes of a photonic crystal, and the properties of space-charge waves on an electron beam, separately.

\subsection{Photonic crystals}

Photonic crystals are structures in which the dielectric constant varies periodically on the order of the wavelength. One distinguishes different types of photonic crystals depending on the number of dimensions that show discrete translational symmetry. An example of a one-dimensional photonic crystal is an infinitely wide stack of an infinite number of periodically alternating layers of two dielectrics. Such a structure has a discrete translational symmetry in one direction and is homogeneous and infinitely extended in the other two dimensions. Analogously, two- and three-dimensional crystals are periodically structured in two and three dimensions. The electromagnetic field distribution allowed in these kinds of structures is strongly influenced by the specific geometry of the crystal. Controlling the design of such crystals enables an unprecedented control over the propagation [17] and emission of light [16]. For a pFEL in particular it turns out that certain spatial Fourier components of the electromagnetic field that co-propagate with the electrons can serve to 
enable stimulated emission.

To calculate the field distribution inside photonic crystals we closely follow the approach of Joannopoulos [44]. In the absence of free charges, such as free electrons, the space charge density $\rho(\mathbf{r}, t)$ and the current density $\mathbf{J}(\mathbf{r}, t)$ are both equal to zero. Under these assumptions Maxwell's equations (2.2)-(2.5) become linear differential equations. The standard approach to solve the remaining equations is to separate the time dependence from the spatial dependence by expanding the field into a set of temporally harmonic modes with the angular frequency $\omega$,

$$
\begin{aligned}
\mathbf{E}(\mathbf{r}, \mathbf{t}) & =\hat{\mathbf{E}}(\mathbf{r}) \exp (i \omega t) \\
\mathbf{H}(\mathbf{r}, \mathbf{t}) & =\hat{\mathbf{H}}(\mathbf{r}) \exp (i \omega t),
\end{aligned}
$$

from which one derives a wave equation for the magnetic field distribution, $\hat{\mathbf{H}}(\mathbf{r})$, given by

$$
\hat{\theta} \times \hat{\mathbf{H}}(\mathbf{r})=\left(\frac{\omega}{c}\right)^{2} \hat{\mathbf{H}}(\mathbf{r})
$$

with $\hat{\theta}$ being defined as the differential operator

$$
\hat{\theta} \equiv \nabla \times\left(\frac{1}{\epsilon(\mathbf{r})} \nabla \times\right)
$$

Equation (2.12) is called the master equation, because it completely determines the magnetic fields inside the photonic crystal if the magnetic field distribution $\hat{\mathbf{H}}(\mathbf{r}, \mathbf{t})$ is chosen such that it is orthogonal to the electric field distribution $\hat{\mathbf{E}}(\mathbf{r}, \mathbf{t})$ [44]. The strategy to find this orthogonal pair of electric and magnetic field distributions, for a structure with a given $\epsilon(\mathbf{r})$ is as follows. First one solves for the magnetic field distribution $\hat{\mathbf{H}}(\mathbf{r}, \mathbf{t})$ via eq. (2.12). Then one computes the corresponding, orthogonal electric field distribution by using eq. (2.2)

$$
\hat{\mathbf{E}}(\mathbf{r})=-\frac{i}{\omega \epsilon_{0} \epsilon(\mathbf{r})} \nabla \times \hat{\mathbf{H}}(\mathbf{r})
$$

When inspecting the master equation (2.12) in more detail, one observes that the master equation defines an eigenvalue problem, which defines certain eigenvalues and eigenvectors. The eigenvalues are proportional to the square of the angular frequency $\omega$. To each frequency $\omega$, for which a solution of the master equation exists, corresponds an eigenvector which is a particular spatial magnetic field distribution. Via eq. (2.14) this defines also 
a particular electric field distribution. Together, the pair of the two field distributions forms a certain eigenmode of the photonic crystal which we also denote in the following as photonic crystal mode.

Recognizing the master equation as an eigenvalue problem has two important advantages. First, numerous numerical methods exist for eigenvalue problems which can be applied to solve for the eigenmodes [121]. Second, by looking at the mathematical properties of the operator $\hat{\theta}$, one can derive some general characteristics of eigenmodes of photonic crystals without the need to specify a particular geometry. Examples are symmetry and scaling properties. The latter leads to a very useful and simple relationship between situations which differ only in the overall spatial scale but not in the spatial structure of the crystal.

To derive this useful scaling relation we consider a photonic crystal with a given spatial dielectric configuration, $\epsilon(\mathbf{r})$. Let $\hat{\mathbf{H}}_{\mathbf{a}}(\mathbf{r})$ be a specific solution of the master equation with an eigenfrequency $\omega_{a}$. Accordingly, the master equation for that photonic crystal mode is

$$
\nabla \times\left(\frac{1}{\epsilon(\mathbf{r})} \nabla \times \hat{\mathbf{H}}_{\mathbf{a}}(\mathbf{r})\right)=\left(\frac{\omega_{a}}{c}\right)^{2} \hat{\mathbf{H}}_{\mathbf{a}}(\mathbf{r}) .
$$

Now, we spatially compress (or expand) the dielectric configuration of the initial photonic crystal using an arbitrary scaling factor $s$, to create a new photonic crystal and find its eigenmodes. The scaled dielectric dielectric configuration, $\epsilon^{\prime}(\mathbf{r})$, can be described by using the scaling factor to modify the spatial coordinates of the initial dielectric configuration,

$$
\epsilon^{\prime}(\mathbf{r})=\epsilon(\mathbf{r} / s)
$$

To find the eigenmode field of the scaled photonic crystal we perform a substitution of variables in equation (2.15). We set $\mathbf{r}^{\prime}=s \mathbf{r}$ and $\nabla^{\prime}=\nabla / s$, which yields

$$
s \nabla^{\prime} \times\left(\frac{1}{\epsilon\left(\mathbf{r}^{\prime} / s\right)} s \nabla^{\prime} \times \hat{\mathbf{H}}_{\mathbf{a}}\left(\mathbf{r}^{\prime} / s\right)\right)=\left(\frac{\omega_{a}}{c}\right)^{2} \hat{\mathbf{H}}_{\mathbf{a}}\left(\mathbf{r}^{\prime} / s\right) .
$$

Here, $\epsilon\left(\mathbf{r}^{\prime} / s\right)$ can be replaced by $\epsilon^{\prime}\left(\mathbf{r}^{\prime}\right)$, see (2.16). Dividing by $s^{2}$ shows that

$$
\nabla^{\prime} \times\left(\frac{1}{\epsilon^{\prime}\left(\mathbf{r}^{\prime}\right)} \nabla^{\prime} \times \hat{\mathbf{H}}_{\mathbf{a}}\left(\mathbf{r}^{\prime} / s\right)\right)=\left(\frac{\omega_{a}}{c s}\right)^{2} \hat{\mathbf{H}}_{\mathbf{a}}\left(\mathbf{r}^{\prime} / s\right) .
$$

It can be seen that this equation is identical to the master equation except that the new eigenmode, $\hat{\mathbf{H}}_{\mathbf{b}}\left(\mathbf{r}^{\prime}\right)=\hat{\mathbf{H}}_{\mathbf{a}}\left(\mathbf{r}^{\prime} / s\right)$, is scaled and the associated frequency, $\omega_{b}=\omega_{a} / s$, is scaled by the same factor. To retrieve the mode profiles and frequencies for a scaled dielectric 
configuration $\epsilon^{\prime}$ of a photonic crystal one can simply re-scale the old mode profiles and frequencies. This is called the scale-invariance of Maxwell's equations.

The implications of the scale-invariance of Maxwell's equations are enormous, both for experimental realizations and for theoretical descriptions [44]. For instance, the fabrication of photonic crystals at optical wavelengths is very complex, time consuming and prone to relatively large fabrication errors. However, for example in the microwave regime, where the electromagnetic wavelengths are much larger, photonic crystals can be easily fabricated. The scaling behavior allows the basic properties of a particular photonic crystal to be studied at the microwave range and then the obtained results can be scaled to the optical wavelength range. The only restriction is that materials with the same dielectric coefficient have to be applied at both corresponding frequencies. Typically, this is possible for many materials. For example, the high refractive index of silicon at optical wavelengths is quite comparable to the high index of aluminun oxide in the microwave range. Also, for theoretical calculations the scale invariance is very important. Regardless of the precise practical dimensions the computed field distribution for a certain geometry can be scaled to any frequency. This obviously reduces numerous, time consuming computations for the same geometry of photonic crystals at different wavelength scales, but it also allows treating the numerical problem dimensionless. This greatly reduces the required effort to develop an appropriate numerical algorithm.

When applying such algorithms to a specific photonic crystal, e.g., to develop appropriate photonic crystals for pFELs, it is found that the collection of eigenfrequencies forms a band structure for the photonic crystal. This is in analogy to the band structure found for electrons in solid-state matter. Many other properties of electron bands in a crystal can also be found for photons in a photonic crystal, such as bandgaps. For photons a bandgap is a frequency range in which no eigenmode can be found for any propagation direction or polarization. Besides identifying the meaning of bandgaps, applying knowledge from solid-state physics to other aspects of photonic crystals turns out to be very insightful, especially for understanding the spatial shape and symmetry of electromagnetic field distributions.

In the theory of solid-state physics, many properties of a crystal are deduced by assuming that the crystal is infinitely extended into all directions. In this case all properties can be obtained by simply studying the unit cell of the crystal $[122,123]$. Analogue to solid-state physics it is possible to define a unit cell of the photonic crystal and to associate reciprocal lattice vectors

$$
\mathbf{G}=m_{1} \mathbf{b}_{\mathbf{1}}+m_{2} \mathbf{b}_{\mathbf{2}}+m_{3} \mathbf{b}_{\mathbf{3}}
$$




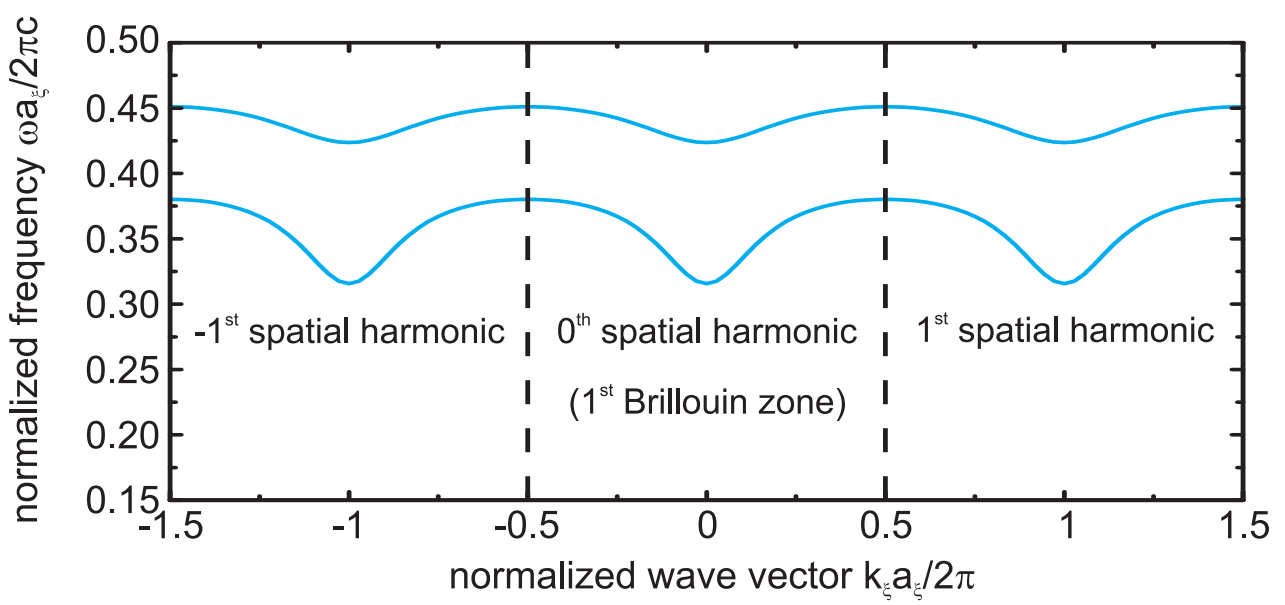

Figure 2.2: The photonic band structure of a photonic crystal in a particular crystal direction $\xi$. Two photonic crystal modes are shown in a normalized wave vector range of the $-1^{\text {st }}$ to $1^{\text {st }}$ spatial harmonic. For completeness the definition of the $1^{\text {st }}$ Brillouin zone, originating from solid-state physics is also indicated.

Here, $m_{1}, m_{2}$ and $m_{3}$ are integers and $\mathbf{b}_{\mathbf{1}}, \mathbf{b}_{\mathbf{2}}$, and $\mathbf{b}_{\mathbf{3}}$ are the primitive unit vectors that span up reciprocal space. By exploiting the consequences of translational symmetry in all directions for the master equation it can be shown that the eigenmodes of photonic crystals $\hat{\mathbf{H}}\left(\mathbf{r}, \mathbf{k}_{\mathbf{0}}\right)$ can be expressed as

$$
\hat{\mathbf{H}}\left(\mathbf{r}, \mathbf{k}_{\mathbf{0}}\right)=\mathbf{u}\left(\mathbf{r}, \mathbf{k}_{\mathbf{0}}\right) \exp \left(-i \mathbf{k}_{\mathbf{0}} \mathbf{r}\right)
$$

This means that the electromagnetic field of each photonic crystal mode can be seen as a single plane wave with the wave vector $\mathbf{k}_{\mathbf{0}}$ multiplied by a periodic vector function $\mathbf{u}\left(\mathbf{r}, \mathbf{k}_{\mathbf{0}}\right)$. This result is commonly known in solid-state physics as Bloch's theorem [122], and in electrical engineering as Floquet theorem [124]. It is of major importance to recognize that $\mathbf{u}\left(\mathbf{r}, \mathbf{k}_{\mathbf{0}}\right)$ is equal to $\mathbf{u}\left(\mathbf{r}, \mathbf{k}_{\mathbf{0}}+\mathbf{G}\right)$. Thus, the frequency corresponding to $\mathbf{k}_{\mathbf{0}}$ is the same for $\mathbf{k}_{\mathbf{0}}+\mathbf{G}$. Figure 2.2 illustrates this with the example of a specific photonic crystal by plotting the frequencies of two eigenmodes along a particular direction $\xi$ in which the period length of the crystal is $a_{\xi}$. From the figure it is observed that the dispersion, which is the variation of $\omega$ versus $k_{\xi}$, is periodic. This means that the full information of a band diagram can be limited to the so-called $1^{\text {st }}$ Brillouin zone or $0^{\text {th }}$ spatial harmonic, with $-a_{\xi} / \pi<k_{\xi, 0}<a_{\xi} / \pi$. Both names have the same meaning, but originate from solid-state physics [122] or electrical engineering [124], respectively. For the remainder of this thesis we chose the terminology of electrical engineering and use the term spatial harmonic.

The function $\mathbf{u}\left(\mathbf{r}, \mathbf{k}_{\mathbf{0}}\right)$ itself can be expressed as an infinite series of weighted plane 
waves and eq. (2.20) becomes

$$
\hat{\mathbf{H}}\left(\mathbf{r}, \mathbf{k}_{\mathbf{0}}\right)=\sum_{\mathbf{G}=-\infty}^{\infty} \mathbf{c}_{\mathbf{G}}\left(\mathbf{k}_{\mathbf{0}}\right) \exp \left[-i\left(\mathbf{k}_{\mathbf{0}}+\mathbf{G}\right) \mathbf{r}\right]
$$

Each component of the series is called a spatial harmonic and $\mathbf{c}_{\mathbf{G}}\left(\mathbf{k}_{\mathbf{0}}\right)$ is the vector of amplitudes for that spatial harmonic. The wave vectors of each spatial harmonic must lie on reciprocal lattice points. In eq. (2.21) this is expressed by stating that the summation over $\mathbf{G}$ extends from minus to plus infinity. In other words, the summation extends over the complete reciprocal space. It should be noticed that only the complete sum is an eigenmode of the photonic crystal and not each spatial harmonic separately.

In the remainder of this thesis, the periodicity of photonic crystals is considered, for simplicity, only along the most important direction. This is the direction along which the electron beam propagates ( $z$-direction). In this specific case the electric field $\hat{\mathbf{E}}$ is

$$
\begin{aligned}
& \hat{\mathbf{E}}\left(\mathbf{r}, k_{z, 0}\right)=\sum_{m=-\infty}^{\infty} \mathbf{E}_{m}\left(x, y, k_{z, 0}\right) \exp \left(-i k_{z, m} z\right) \\
& \text { with } k_{z, m}=k_{z, 0}+\frac{2 \pi}{a_{z}} m .
\end{aligned}
$$

Here $a_{z}$ is the period length of the unit cell in the $z$-direction and $m$ is the order of the spatial harmonic.

To connect these theoretical findings to the operation of pFELs, let us discuss the consequences of the properties of photonic crystal modes for the emission of Čerenkov radiation. In homogeneous dielectrics the emission of Čerenkov radiation requires that the electron beam velocity exceeds the phase velocity of the electromagnetic field along the electron propagation direction, here the $z$-direction. The phase velocity along the $z$-direction is $\omega / k_{z}$. While electromagnetic fields in homogeneous dielectrics contain only a single wave number $k_{z}$, photonic crystal modes consist of an infinite number of spatial harmonics. Each spatial harmonic has a different wave number $k_{z, m}$. Thus, each spatial harmonic also has its own specific phase velocity $v_{p h, m}$. A consequence of this is that Čerenkov radiation inside photonic crystals shows no velocity threshold [119], unlike Čerenkov emission in bulk dielectrics. For any electron velocity there always exists a spatial harmonic with a phase velocity lower than the electron velocity. The spatial harmonic wave number $k_{z, m}$ approaches infinity for large $m$. Hence, the associated phase velocity approaches zero. However, for the operation of a pFEL this turns out to be of less importance. At low electron velocities only a small probability for emission is found when studying single electrons streaming through photonic crystals. This can be seen in the 
derivation of Kremers, who shows that the emission probability for Čerenkov radiation by a single electron decreases with the spatial harmonic amplitude $\mathbf{E}_{m}\left(x, y, k_{z, 0}\right)$ [125]. As eq. (2.22) is a Fourier series, the spatial harmonic amplitudes $\mathbf{E}_{m}\left(x, y, k_{z, 0}\right)$ converge towards zero when $m$ reaches high values. Thus, also the emission probability strongly reduces. From this we deduce that for a pFEL operation in a low spatial harmonic is desired for having a low current threshold.

\subsection{Electromagnetic waves on electron beams}

Besides in photonic crystals, electromagnetic waves can also propagate in a wide variety of other systems, including electron beams [88, 89, 126-128]. An electron beam consists of a large number of electrons confined in a certain volume. Such confinement can be achieved with a strong magnetic field. Owing to the long-range nature of electrostatic interactions between the individual electrons, collective motion of the electrons allows for propagation of waves along an electron beam, so-called space-charge waves. Spacecharge waves are longitudinal waves that involve a spatio-temporal modulation of the electron density. Such motion affects the velocity-matching between the electrons and the electromagnetic waves inside the crystal and are crucial for an understanding of the specific frequency at which amplification occurs in slow-wave FELs [89].

To qualitatively explain the origin of space-charge waves, let us consider an infinitely extended electron beam. The electrons are initially at rest and the total charge is neutralized by a gas of positively charged, immobile ions. At equilibrium the electrons are distributed homogeneously throughout the entire beam. However, a local imbalance of charge can be created, e.g., by taking a sheet of electrons and displacing them from their equilibrium position. This creates an electric field that pulls the electrons back to their equilibrium position. When the electrons reach the equilibrium position they still have momentum and overshoot, similar to the well-known harmonic oscillator. This process repeats periodically and leads to an oscillation of the electrons around their equilibrium positions. The oscillation frequency of the motion is the so-called plasma frequency, $\omega_{p}$, and can be calculated via [89]:

$$
\omega_{p}=\sqrt{\frac{e^{2} n_{e l}}{m_{e l} \epsilon_{0}}} .
$$

Here $n_{e l}$ is the electron number density. While an oscillation of a lump of electrons around the equilibrium position might be expected to induce a propagating wave via coupling to neighboring electrons, in a stationary electron beam this is not found. It turns out that 
the described oscillation corresponds to a dispersion relation that is independent of the wave number $k$ and, therefore, has a group velocity of $v_{g}=0$. Thus, no propagating spacecharge wave is induced on a stationary electron beam by disturbing its local equilibrium. This does also not change if the electron beam travels in a certain direction. Nevertheless, a stationary observer would perceive the local space-charge oscillation as a propagating space-charge wave.

The complete description of the dynamics of a traveling electron beam is much more complex. In order to find a quantitative solution for space-charge waves on electron beams we summarize the results given by Gilmour [89]. Mathematically, space-charge waves are described by the coupled Maxwell's and Newton-Lorentz equations. To find the properties of space-charge waves Gilmour derives the following wave equation from the Maxwell's equation [89]

$$
\nabla^{2} \mathbf{E}-\frac{1}{c^{2}} \frac{\partial^{2} \mathbf{E}}{\partial^{2} t}=\mu_{0} \frac{\partial \mathbf{J}}{\partial t}+\nabla\left(\frac{\rho}{\epsilon_{0}}\right)
$$

Here it is assumed that $\epsilon$ is unity, which expresses the relatively low particle densities in a typical beam. Further, it is assumed that the electron beam travels in the positive $z$-direction and that initially all electrons have the same velocity, $v_{z, 0}$. To focus only on the basic nature of space-charge waves, we again assume an infinitely extended electron beam which is neutralized by a background gas of stationary, positively charged ions. Mathematically this means that we perform a one-dimensional analysis, where transverse variations are excluded. Physically such a situation occurs when an infinite magneto-static field is applied along the $z$-direction, such that the Lorentz force restricts the electron motion to the $z$-direction. In this one-dimensional treatment the vectors $\mathbf{E}$ and $\mathbf{J}$ in eq. (2.25) become scalars, $E_{z}$ and $J_{z}$, and one obtains a scalar wave equation

$$
\frac{\partial}{\partial z} E_{z}-\frac{1}{c^{2}} \frac{\partial^{2} E_{z}}{\partial^{2} t}=\mu_{0} \frac{\partial J_{z}}{\partial t}+\frac{\partial}{\partial z}\left(\frac{\rho}{\epsilon_{0}}\right)
$$

Even with these significant approximations, can eq. (2.26) not easily be solved. The differential equation (2.26) remains nonlinear because the current density, which enters eq. (2.26), is the product of electron velocity and the space-charge density:

$$
J_{z}(z, t)=\rho(z, t) v_{z}(z, t)
$$

To find the solution of eq. (2.26), one can linearize the system of coupled equations when restricting to small changes of all oscillating quantities as compared to their stationary values. Thus, for the electric field $E_{z}$, the current density $J_{z}$, the space-charge density $\rho$, 
and the electron velocity $v_{z}$ we write

$$
A(z, t)=A_{0}(z)+\tilde{A}_{R F} \exp \left(i \omega t-i k_{z} z\right) \quad \text { with } \tilde{A}_{R F} \ll A_{0} .
$$

Here, the $A$ represents the quantities, $E_{z}, J_{z}, \rho$, and $v_{z}$. Note that this approach removes from the considerations the stationary part of the field (DC-component) caused by the average space-charge of electrons. The remaining dynamical part (RF-component) oscillates in time and space, and forms the space-charge wave. For the amplitude of the oscillating beam current $\tilde{J}_{z, R F}$, the result of the linearization can be summarized as the so-called electronic equation [89],

$$
\tilde{J}_{z, R F}=-i \omega \epsilon_{0} \frac{\omega_{p}^{2}}{\gamma^{3}\left(\omega-k_{z} v_{z, 0}\right)^{2}} \tilde{E}_{z, R F}
$$

Inserting the electronic equation into the wave equation (2.26), we derive the following dispersion equation,

$$
\left(k_{z}^{2}-\frac{\omega^{2}}{c^{2}}\right)\left(1-\frac{\omega_{p}^{2}}{\gamma^{3}\left(\omega-k_{z} v_{z, 0}\right)^{2}}\right) \tilde{E}_{z, R F}=0 .
$$

This equation has four independent solutions, two being ordinary electromagnetic waves with $k_{z}= \pm \omega / c^{2}$ which would also exist without an electron beam present: i.e. at zero plasma frequency $\omega_{p}=0$. The other two solutions are only possible with an electron beam present and are space-charge waves. From eq. (2.30) the wave numbers of these space-charge waves are found as

$$
k_{z}=\frac{\omega \mp \omega_{p} / \gamma^{3 / 2}}{v_{z, 0}}
$$

The meaning of this equation can be better understood by calculating the phase velocities of the two waves it describes,

$$
\begin{aligned}
& v_{\text {fast }}=\frac{v_{z, 0}}{1-\gamma^{-3 / 2} \omega_{p} / \omega} \\
& v_{\text {slow }}=\frac{v_{z, 0}}{1+\gamma^{-3 / 2} \omega_{p} / \omega} .
\end{aligned}
$$

It can be seen that the space-charge waves which can propagate along an electron beam either have a phase velocity slightly faster or slower than the DC velocity of the electron beam. Hence, the modes are named fast space-charge wave or slow space-charge wave, respectively. 
The consequence of the excitation of space-charge waves can be illustrated by comparing the kinetic energy density of an electron beam of uniform velocity $v_{z, 0}$ [128],

$$
u=m_{e l} c^{2} n_{e l}(\gamma-1)
$$

to the energy density when space-charge waves are present.

It can be shown [128] that in the latter case the energy density, $u$, is increased by an amount

$$
\Delta u_{\text {fast }}=\frac{1}{2} \frac{m_{e l}}{e}\left|\tilde{\rho}_{R F}\right|^{2} \gamma^{3} \frac{v_{z, 0}}{\rho_{0}}\left(v_{\text {fast }}-v_{z, 0}\right)>0,
$$

for the fast space-charge wave, and the energy density is lowered for the slow space-charge wave by

$$
\Delta u_{\text {slow }}=\frac{1}{2} \frac{m_{e l}}{e}\left|\tilde{\rho}_{R F}\right|^{2} \gamma^{3} \frac{v_{z, 0}}{\rho_{0}}\left(v_{\text {slow }}-v_{z, 0}\right)<0
$$

This result shows that exciting a slow space-charge wave on an electron beam with an external longitudinal electric field leads to a reduced kinetic energy for the electron beam. Note that, in order to provide a propagating electromagnetic wave that can reduce the kinetic energy a wave needs to have a longitudinal field component. This requires appropriate boundary conditions [129]. If these boundary conditions are met, the reduction of kinetic energy will lead, via total energy conservation, to a growth of the inducing longitudinal electric field. Assume now a weak longitudinal field is initially present. This weak field then excites a space-charge wave that excites a larger electric field which excites again a space-charge wave and so on. As a result an energy flow from electron beam to electromagnetic wave sets in and increases the initially low electromagnetic field amplitude. In contrast, exciting the fast space-charge wave increases the kinetic energy as compared to the original electron beam. Energy from the electric field is transferred to the electron beam, which lead to an energy flow from electromagnetic wave to the electron beam, which reduces the electromagnetic field amplitude to increase the electron velocity. For the pFEL this means that only the slow space-charge wave can grow in the interaction between the electrons and the velocity-matched mode component in the photonic crystal. The fast space-charge wave is still of interest, but for other applications. For instance, for the purpose of building an electron accelerator by injecting an external electromagnetic wave into a photonic crystal [130-133]. Photonic crystal accelerators are, however, beyond the scope of this thesis. 


\subsection{Linear theory of slow-wave free-electron lasers}

In the previous section, two types of wave solutions were found for an infinite electron beam by linearizing the coupled differential equations set up by the Maxwell and NewtonLorentz equations. The resulting dispersion equation (2.30) has four solutions. Two of the solutions were found to be ordinary electromagnetic waves with a phase velocity of $v_{p h}=c$. Two space-charge waves were found to have a phase velocity $v_{p h}=v_{\text {fast }}$ and $v_{p h}=v_{\text {slow }}$, which is close to the DC electron velocity $v_{z, 0}$. In the discussed case, these waves remain uncoupled which is seen by the fact that the dispersion equation (2.30) is homogeneous.

A coupling is introduced between the four wave solutions when the electric field is also allowed to vary in the transverse directions: i.e.

$$
\left(\frac{\partial^{2}}{\partial x^{2}}+\frac{\partial^{2}}{\partial y^{2}}\right) E_{z} \neq 0 .
$$

However, the general result hardly changes if the phase velocity of the electromagnetic waves without an electron beam present remains greatly different from the electron velocity [128]. For example, in free space, or when a material lowers the phase velocity of the electromagnetic waves somewhat, the two electromagnetic waves have a phase velocity in the order of the vacuum velocity of light, $v_{p h} \approx c$, and with a relatively slow electron beam the space-charge waves have their phase velocity $v_{p h} \approx v_{z, 0}$ close to the slow electron velocity. With such a significant velocity mismatch the coupling between electron beam and electromagnetic wave remains very weak.

To create a strong coupling, the phase velocity of the electromagnetic waves needs to be decreased so much that it is close to the slow electron velocity, e.g., by means of a suitable dielectric. To mathematically describe this situation, the electric field, the current density, the space-charge density, and the electron velocity are again linearized using the ansatz of eq. (2.28). However, now the wave vector $k_{z}$ will generally have to be a complex number such that its imaginary part describes the growth of the amplitude with propagation distance $z$. As we have just discussed, for any coupling between electromagnetic waves and electron beams, transverse variations in the fields are needed. Thus, transverse variations should be taken into account in the electronic equation (2.29) and the electronic equation becomes a vector equation. However, when the longitudinal current density $J_{z}$ is much bigger than the transverse current densities $J_{x}$ and $J_{y}$, and, thus $J_{z}$ varies approximately only with $z$, eq. (2.29) remains a good approximation. This is justified when a strong magneto-static guiding field limits the electron motion to the $z$-direction. In this situation 
the wave equation for the $z$-component of the electric field becomes

$$
\left(k_{z}^{2}-\frac{\omega^{2}}{c^{2}}\right)\left(1-\frac{\omega_{p}^{2}}{\gamma^{3}\left(\omega-k_{z} v_{z, 0}\right)^{2}}\right) \tilde{E}_{z, R F}(x, y)=-\nabla_{T}^{2} \tilde{E}_{z, R F}(x, y) .
$$

Here, $\nabla_{T}^{2}=\frac{\partial^{2}}{\partial x^{2}}+\frac{\partial^{2}}{\partial y^{2}}$ is the transverse Laplace operator. For the complex wave number it is assumed that it remains comparable to the wave number of the electromagnetic wave without an electron beam present, i.e.,

$$
k_{z}=k_{z, \epsilon}+\Delta k \quad \Delta k \ll k_{z, \epsilon} \text {. }
$$

Here $k_{z, \epsilon}$ is the wave number of the electromagnetic field inside the dielectric in the absence of an electron beam. The effect of the coupling to the electron beam is described by $\Delta k$. These two equations describe generally the linearized growth of an electric field inside a slow-wave FEL whose field in absence of the electron beam can be described by a single wave number $k_{z, \epsilon}$.

Solving equation (2.38) has been carried out using different approaches [54, 103]. The original solution from Pierce is based on using equivalent transmission lines and a ballistic approach [54]. Later, also direct solutions of the wave equation have been given [103]. As we use very slow electrons, $v_{z, 0} \ll c$, throughout this thesis we can restrict ourselves to the non-relativistic limit with $\gamma=1$. If we further assume velocity-matched amplification by a transversely thin electron beam, i.e., evaluating eq. (2.38) at a single transverse beam location $\left(x_{b}, y_{b}\right)$, both mathematical approaches give the same solution. The four different wave numbers $k_{j}$ that belong to linearly independent solutions of equation (2.38) are

$$
\begin{array}{ll}
k_{1}=k_{z, \epsilon}+\frac{k_{z, \epsilon} C}{2}+i \frac{\sqrt{3}}{2} k_{z, \epsilon} C, & k_{3}=k_{z, \epsilon}-k_{z, \epsilon} C, \\
k_{2}=k_{z, \epsilon}+\frac{k_{z, \epsilon} C}{2}-i \frac{\sqrt{3}}{2} k_{z, \epsilon} C, & k_{4}=-k_{z, \epsilon}+k_{z, \epsilon} \frac{C^{3}}{4},
\end{array}
$$

with $C$ being the so-called gain parameter defined as:

$$
C \equiv\left(\frac{\left|E_{z, \text { matched }}\left(x_{b}, y_{b}\right)\right|^{2}}{2 k_{z, \epsilon}^{2} W v_{g}} \frac{I_{0}}{4 U_{0}}\right)^{1 / 3} .
$$

Here, $I_{0}$ is the electron beam current, $U_{0}$ the kinetic energy of the electrons expressed as the acceleration voltage used to accelerate the electron beam, $W$ is the energy per unit volume stored in the electromagnetic wave, and $v_{g}$ is the group velocity at the velocity-matched wave number. The gain parameter further depends on the electric field 


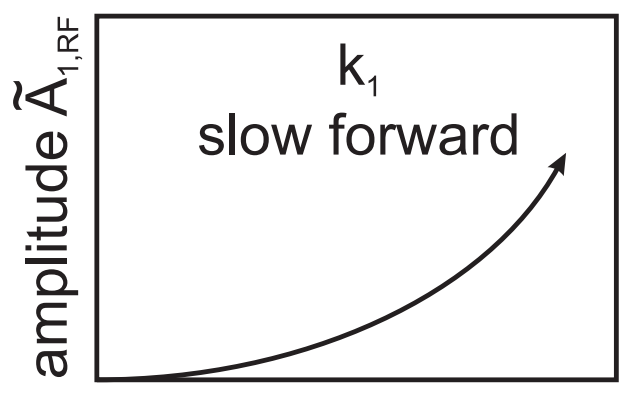

z-position

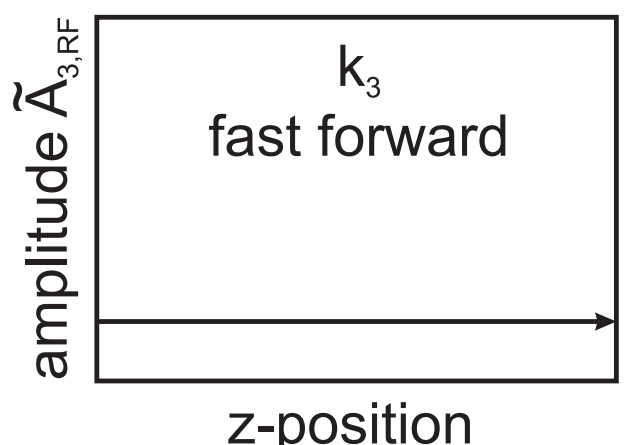

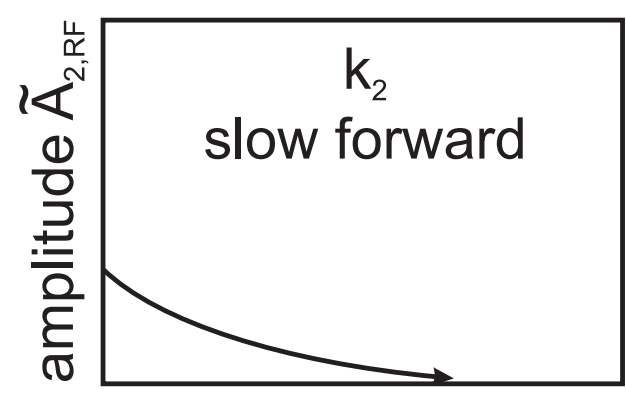

Z-position

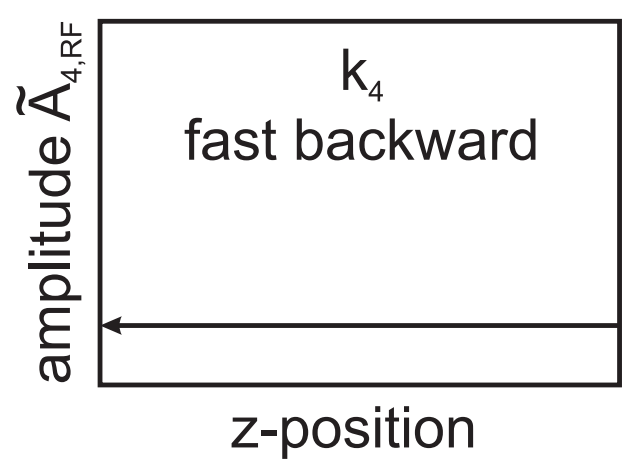

Figure 2.3: The amplitude $\tilde{A}_{j, R F}$ versus $z$-position of the four waves resulting from the coupling of an electromagnetic field with space-charge waves.

amplitude, $E_{z \text {,matched }}\left(x_{b}, y_{b}\right)$, of the $z$-component that is velocity-matched. The observation that four different wave numbers are found as a solution for the differential equation can be understood as follows. While, in a dielectric without an electron beam present, only a single wave number $k_{z, \epsilon}$ describes the electromagnetic field, this fundamentally changes when an electron beam is present. As each $k_{j}$ describes an independent solution of the differential equation (2.38), the general solution for the oscillating part of the electromagnetic field, $\hat{E}_{z, R F}$, and all other quantities $\left(\hat{\rho}_{R F}, \hat{J}_{z, R F}, \hat{v}_{z, R F}\right)$ has to be a linear superposition of all four waves:

$$
\hat{A}_{R F}\left(x_{b}, y_{b}, z\right)=\sum_{j=1}^{4} \tilde{A}_{j, R F}\left(x_{b}, y_{b}\right) \exp \left(-i k_{j} z\right)
$$

Here $A$ represents again any of these quantities. The amplitudes of the specific solutions, $\tilde{A}_{j, R F}$, are determined by the specific boundary and initial conditions of the investigated situation.

In order to understand the physical meaning of the four different wave numbers, $k_{j}$, 
let us discuss the magnitude and sign of their real and imaginary parts. What simplifies the discussion is that the gain parameter $C$ typically ranges between 0.01 to 0.1 , [89]. Thus, the real part of all four wave numbers $k_{j}$ is close to the wave number $k_{z, \epsilon}$ in the absence of the electron beam. The sign of the real part of the wave numbers $k_{1}$ and $k_{2}$ is positive. Hence, the associated waves propagate in the positive $z$-direction (forward). The magnitude of the wave numbers $k_{1}$ and $k_{2}$, is slightly bigger than the original wave number $k_{z, \epsilon}$ which indicates that both waves propagate at the same frequency $\omega$ with a lower phase velocity than the original wave. Analyzing $k_{3}$ and $k_{4}$ we observe that the wave associated with $k_{3}$ propagates faster than the original wave in the forward direction and that the wave with the wave number $k_{4}$ propagates backwards. Sign and magnitude of the imaginary part of the wave numbers $k_{j}$ indicate whether the wave amplitude is constant, exponentially decaying, or increasing with the positive $z$-position. This is schematically depicted in Fig. 2.3 for positive $C$. It can be seen that, for the complex conjugated pair $k_{1}$ and $k_{2}$, one of the wave is exponentially damped and the other is exponentially growing. The growth of the amplitude is hereby dependent on the gain parameter $C$. The wave numbers $k_{3}$ and $k_{4}$ have a zero imaginary part. Thus, their amplitude stays constant with increasing $z$-position.

The gain parameter $C$ can also become negative, namely, when the group velocity of the wave is negative. We will find that this is the situation typically encountered in photonic crystals, but the approach for deriving the wave numbers remains valid [88]. The only difference in the final result is that now, instead of the wave with $k_{1}$, it is the wave with $k_{2}$ which grows exponentially and $k_{1}$ decreases exponentially with position. Thus, depending on the sign of $C$, the wave with the wave number $k_{1}$ or $k_{2}$ should be observed as amplified output.

While it is found that only one of the four possible waves is exponentially growing, in general a non-zero amplitude of the other waves is still required to fulfill the boundary and initial conditions of the differential equation system. The standard approximation is to assume that only the three forward waves are excited [87]. Under the conditions that the incoming beam is initially not bunched, shows no velocity spread, and that a certain non-zero input field, $E_{\text {in }}$, is injected at $z=0$ this input field grows as [89]

$$
\hat{E}_{z, R F}=\frac{E_{i n}}{3}\left[1+2 \exp \left(-i \frac{6 \pi}{2} C N\right) \cosh (\pi \sqrt{3} C N)\right] \exp [-i(1-C) 2 \pi N] .
$$

Here $z$ is expressed in units of the wavelength $\lambda_{z, \epsilon}, N=z \lambda_{z, \epsilon}^{-1}$. Equation (2.43) describes a rather complex development of the injected field with propagation distance. A superposition of three waves, that either grow, decay or remain constant with $z$ is found. The 
named initial and boundary conditions imply that, at $z=0$ or $N=0$, each of these waves carries a third of the total amplitude. Only at a distance of multiple wavelengths, beyond $N \gg 1$, shows eq. (2.43) that the growing wave begins to dominate and the total field can be approximated with a much simpler expression [89]

$$
\hat{E}_{z, R F}=\frac{E_{i n}}{3} \exp (\pi \sqrt{3} C N)
$$

and a corresponding power growth,

$$
\hat{P}_{z, R F}=\frac{P_{i n}}{9} \exp (2 \pi \sqrt{3} C N)
$$

We observe that effectively only a third of the actually injected field amplitude, $E_{i n}$, becomes amplified, while the other two thirds decrease or remain unchanged. The wave that is amplified, due to the factor one third in the initial field amplitude, carries only a ninth of the injected power as can be seen in the eq. (2.45). Therefore, only a one ninth fraction of the initial power, $P_{i n}=E_{i n}^{2}$, becomes effectively amplified. An simplified physical understanding of this mathematical conclusion is as follows. To bunch the initially continuous stream of electrons, half of the electrons needs to be accelerated which decreases a part of the initially injected field strength.

It is common to express eq. (2.45) in terms of a power gain in decibel:

$$
G[\mathrm{~dB}]=-9.54[\mathrm{~dB}]+47.3 C N[\mathrm{~dB}]
$$

The $-9.54 \mathrm{~dB}$ term results from the factor one ninth in eq. (2.45) and can be seen as an effective loss factor for radiation injected at $z=0$ for amplification. As the loss term is connected with the initial absence of bunching in the injected electron beam, we will refer to this term as bunching loss in the remainder of this thesis. The value of the second term per number of wavelengths, $G_{\lambda}=47.3 C$, is usually called gain per wavelength.

So far we have investigated thin electron beams, however, in real situations the electron beam always possesses a finite transverse size. This means that transversely different parts of the electron beam experience a varying $E_{z}$ field. To take this transverse variation into account an averaging over the electron beam cross section, $S$, is usually performed to determine $C$ :

$$
C=\left(\frac{\int_{S}\left|E_{z, \text { matched }}(x, y)\right|^{2} \mathrm{~d} \mathbf{S}}{2 k_{z, \epsilon}^{2} W v_{g} S} \frac{I_{0}}{4 U_{0}}\right)^{1 / 3}
$$

In summary, eqs. (2.46) and (2.47) predict the small-signal gain experienced by an elec- 
tromagnetic wave when driven by an electron beam of matched velocity. The wave is considered to contain just a single wave number $k_{z, \epsilon}$ in the absence of the electron beam. While this is a valid assumption for an electromagnetic wave propagating inside a dielectric we have seen that a photonic crystal mode consists of a superposition containing multiple wave numbers, so-called spatial harmonics. The amplitude of these spatial harmonics, $\mathbf{E}_{m}\left(x, y, k_{z, 0}\right)$, is determined from the specific spatial boundary conditions which the total field has to comply to in a given type of photonic crystal, compare eq. (2.22).

This means that the above theory does not represent the precise amplification conditions found in a photonic crystal. While the theory describes the amplification for a single wave number only, multiple wave numbers should be included in the ansatz when solving the differential equation (2.38). Such a situation is also present in backward-wave oscillators that use corrugated waveguides for velocity-matching between the electron beam and the electromagnetic wave [95, 99, 134, 135]. Corrugated waveguides are metallic waveguides with a periodic variation of the transverse size. This geometry leads to an electromagnetic field that is composed of an infinitive number of spatial harmonics. In these devices it is assumed that the interaction takes place only with the velocity-matched wave number of a certain spatial harmonic, for which

$$
k_{z, \epsilon}=k_{z, m}
$$

and that only the electromagnetic field at that wave number bunches the electron beam

$$
E_{z, \text { matched }}(x, y)=E_{z, m}\left(x, y, k_{z, 0}\right) .
$$

This corresponds to the assumption that the corrugated waveguide can be approximated as an effective dielectric medium. The refractive index of this effective dielectric is chosen such that it results in a phase velocity matched to the electron velocity and that the electromagnetic field has a transverse pattern identical to the velocity-matched spatial harmonic. While this assumption is widely used for backward-wave oscillator, it has also been shown that the influence of lower spatial harmonics can either increase or decrease the starting current of backward-wave oscillators [136].

In principle, one can assume the same for a photonic crystal. However, it is not guaranteed that this predicts the actual small-signal gain for a pFEL, where there is also a transverse periodic variation present different to a backward-wave oscillator. Thus, before drawing conclusions for pFELs from this theory we should carefully validate its applicability for pFELs. In addition, truly nonlinear effects, such as the strength of mode competition effects, which involve a strong degree of gain saturation can of course never 
be described by a linear model and require a nonlinear theory.

\subsection{Nonlinear theory: particle-in-cell calculations}

The linear theory presented in the last section can be used to investigate the small-signal gain after being validated. This underlines the need for a more comprehensive approach in order to perform this validation. However, more importantly even the best linear theory will never allow the investigation of truly nonlinear effects in a pFEL. An example of these effects is mode competition. Another very important drawback of linear theories based on photonic crystal modes is that they cannot easily handle the effects that are introduced by additional non-periodic boundaries, such as the finite size of any photonic crystal structure. Investigating these effects is only possible using a theoretical approach that solves the full nonlinear differential equation system (2.2)-(2.8) which can handle all complex geometrical boundary conditions.

Particle-in-cell (PIC) techniques can directly solve the underlying nonlinear differential equation system with very little approximate assumptions [108, 109]. For instance, PIC methods have already been in use since the early 1950th to analyze nonlinear effects in plasma physics [137]. Since then, these methods have developed enormously. Initially only one-dimensional codes where used [137], but now many three-dimensional codes are available [110, 138-141]. These codes have been successfully applied in a huge variety of situations. Examples are found in astrophysics, particle acceleration and FELs $[69,83,142-146]$. For FELs in particular, these codes allow the investigation of multidimensional effects and highly nonlinear phenomena, which is very complex using other techniques [144]. For example, even the most sophisticated analytic codes typically consider the electron beam charge being neutralized by a background of ions [144]. However, a background of ions is typically not present in real FELs, and in contrast to most analytical techniques PIC calculations can easily include this situation.

In FEL applications the PIC method follows the trajectories of the electrons in time and space, dependent on externally applied or self-generated electromagnetic fields in a self-consisted manner. The entire spatial field distribution is computed for each time step on a fixed grid and the individual particle positions and velocities are tracked in a continuous space. Thus, the state of an electron at an instant in time is set by three spatial coordinates and three momentum coordinates represented by a point in a six-dimensional space, the so-called phase space. At each time step the field changes due to new particle positions and due to changing particle velocities via the Maxwell equations. Particle motion and acceleration is calculated based on the field generated forces determined by 


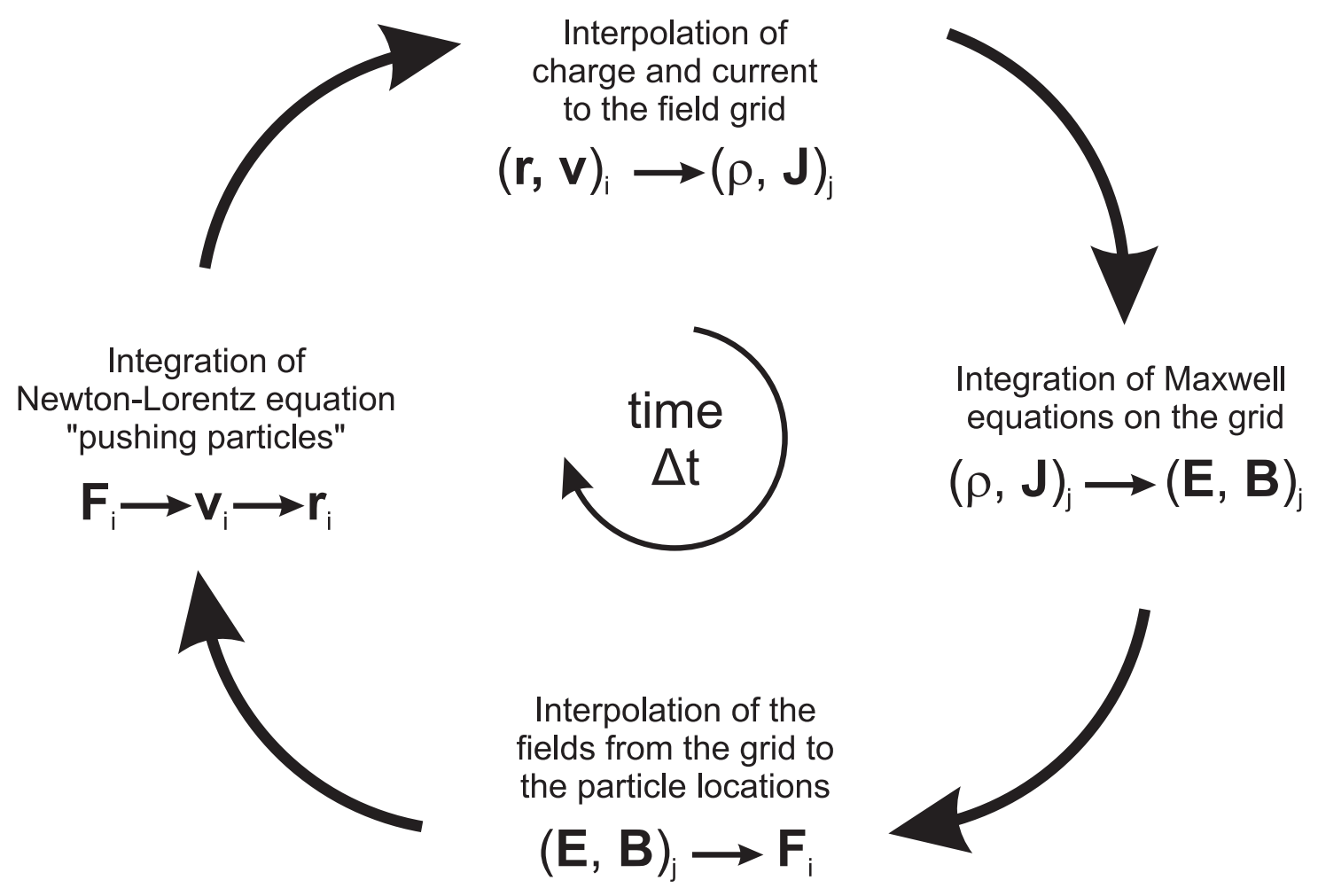

Figure 2.4: The four steps performed during each calculation cycle $\Delta t$ of the particle-incell method. The named equations and symbols are given in section 2.3.

the Newton-Lorentz equation, to proceed to the next time step. Implementation of such calculations in three dimensions has to be carefully designed [109], due to susceptibility to discrete particle noise [147]. Simultaneously, the numerical code needs to be strongly optimized with regard to processing efficiency, so that tolerable computation times can be achieved. A description of the numerical implementation would go far beyond the scope of this thesis. However, the basic implementation of this scheme in only one spatial dimension is conceptually very simple and provides a good insight into the strength of the method.

To simplify the explanation, we discuss here the implementation of a one-dimensional PIC code given by Birdsall and Langdon [109]. The interested reader can find the extension to three-dimensional codes including electromagnetic fields in this excellent textbook as well. The computation cycle used to advance the particles by a single time step $\Delta t$ is shown in Fig. 2.4. The cycle starts with appropriate initial conditions for each particle $i$ at its position $z_{i}$ and velocity $v_{i}$ in the continuous phase space. The field quantities are computed on a spatial grid, $Z_{j}$, at discrete locations in space, as indicated by the index $j$. In a typical FEL about $10^{6}$ to $10^{10}$ electrons are simultaneously present in the laser and need to be processed each time step. While computer technology has made 
impressive advances in the past, such a high number of particles can still not be efficiently processed. To enable a reasonably fast computation it is common to lump together a number of particles into so-called super-particles. These super-particles carry a charge and mass much larger than that of a single electron in a manner that charge and mass flow are equal to the considered electron beam and the stream of super-particles yields the resulting electric current.

To calculate the field generated by the super-particles one needs to determine the current density and space-charge density due to the particles on the grid points. As the particle position can also be located in-between the grid points, this process of charge and current assignment requires an interpolation. As the number of super-particle is still around 100,000 a fast weighting technique is preferable. The fastest weighting technique would be to assign the complete particle charge to the nearest grid point. However, such zero-order weighting tends to advance unphysical results [109]. For a compromise between speed and accuracy, the following first order weighting scheme is typically applied to calculate the charge from each super-particle $i$ on the grid point $j$.

$$
q_{j}=q_{s, i} \frac{\Delta z-\left(z_{i}-Z_{j}\right)}{\Delta z}
$$

Here $\Delta z$ denotes the mesh resolution and $q_{s, i}$ is the charge of the $i^{\text {th }}$ super-particle. Note that an assignment of a point charge to its nearest grid point by linear interpolation produces the same result. Such a viewpoint is named particle-in-cell which has become synonymous for the complete method.

After integration of the field equations on the grid, a second interpolation step is required in order to propagate (push) the particles via the appropriate force present at the particle positions $i$ in the last step. In order to avoid so-called self-forces, it is desirable to use the same weighting scheme for both the field and charge weighting steps [109]. A self-force is a force on a mesh created by a certain particle that in turn acts back on that particle, e.g., accelerating the particle itself. As a first order weighting scheme is used for interpolating the charges on the grid, the same weighting scheme is applied for interpolating the electric field at the particle position $z_{i}$ :

$$
E\left(z_{i}\right)=\left[\frac{Z_{j+1}-z_{i}}{\Delta z}\right] E_{j}+\left[\frac{z_{i}-Z_{j}}{\Delta z}\right] E_{j+1}
$$

To finish the computation cycle, one needs to integrate the equations of motion. From tracing single particles it is known that higher order methods, such as the fifth order Runge-Kutta method, are very accurate [148]. However, the choice of method is strongly influenced by concerns over the computation power required. In the PIC calculations that 
we typically perform here, we select on the order of 100,000 super-particles in the model. The minimum information needed to integrate the equations of motions are the particle positions $z_{i}$ and velocities $v_{i}$ at a single time $t$ for zero order methods. Higher-order methods also require to store also the positions and velocities at earlier times, i.e., at multiple time steps. This increases both the amount of storage required and the number of operations to advance the particles [149]. So it is desirable to find a simple method with a reasonable accuracy.

A simple but astonishingly accurate method is the leap-frog integration scheme [150] which is the standard method employed in PIC schemes to push the particles [109]. After the particles have been pushed, one computational cycle is finished. By repeating a huge number of these computational cycles, each describing an extremely small time step, the particle propagation and generation of fields can be calculated in a self-consistent manner $[108,109]$.

Implementing these steps not only in a single spatial dimension, but in three-dimensions, makes PIC methods very powerful tools. For example, three-dimensional PIC methods allow to compute the temporal evolution of the electromagnetic field, particle positions and velocities in a slow-wave FEL [69, 83, 144, 145]. Specifically for the present work, it becomes possible, e.g., to compute the steady-state output power of pFELs or investigate mode competition in pFELs. This is impossible using linear methods. Further, even sophisticated effects such as space-charge forces from non-neutralized electron beams are included. In the remainder of this thesis, we repeatedly use this powerful method to obtain deeper insight into a pFEL. 



\section{Photonic free-electron laser pumped by a single electron beam}

The emission of light sources is strongly affected by periodic surroundings [16]. This effect is achieved via a modification of the type and density of electromagnetic modes into which emission occurs. Examples are metamaterials, plasmonic structures and photonic crystals. These structures radically alter the emission of embedded light sources, such as quantum dots, molecules and atoms [16, 19, 25, 29, 151, 152]. This control enables unique photonic-crystal lasers, including surface-emitting quantum cascade lasers, ultrafast modulated lasers and ultra-low threshold lasers [31, 32, 34, 35]. Notably, the unique properties of photonic-crystal lasers are also of high interest for many applications, such as optoelectronic integrated circuits [153] and lab-on-a-chip applications.

Lab-on-a-chip applications greatly simplify the analysis of biological and chemical samples [39-43] and photonic-crystal lasers are very attractive as laser source in these applications, because the inherent subwavelength feature size of photonic-crystal lasers allows to supply the radiation in a compact format. A wider applicability of photoniccrystal lasers in these applications requires to provide many different frequency ranges for the great variety of different samples one would like to study. However, to date, photonic-crystal lasers are based on bound-electron transition, e.g., in semiconductors or 
quantum dots, which cannot provide a wide variety of frequencies ranges. Specifically, photonic-crystal lasers have remained limited to the emission of wavelengths that can be amplified by the material-specific transitions provided by the chosen embedded light source [154]. Despite the fact that photonic crystals are widely frequency scalable, see section 2.4, a photonic crystal laser at infrared wavelength, for example based on the emission of quantum dots, cannot be easily scaled to operate at $\mathrm{THz}$ frequencies. Such scaling would require finding embedded light sources at $\mathrm{THz}$ frequencies which provide the same properties as the original light source at optical frequencies. In practice this is very difficult.

In contrast, free electrons emit over huge spectral ranges, when inducing transitions between states of different kinetic energy. This is illustrated by the fact that FEL facilities can cover extremely wide ranges of frequencies from the $\mathrm{THz}$ to the $\mathrm{X}$-ray range [47]. Through the velocity of the electrons the emission frequency is essentially freely scalable, just as is the control of emission via scaling of photonic crystals. Thus, it is of utmost interest to combine the scalable emission frequency of free electrons with the frequency scalability of photonic crystals. A precondition is that the emission of free-electrons can be suitably controlled and enhanced by the photonic crystal in order to realize stimulated emission. Given the frequency scalability of this approach, a smaller photonic crystal period leads to an increased emission frequency. When restricted to relatively low electron velocity, this approach appears promising for the realization of miniaturized free-electron lasers which are hand-held, or even fit "on a chip".

In first attempts of the latter kind, low energy free electrons have been sent through photonic crystals and have shown to generate spontaneous Čerenkov emission in a number of different frequency ranges $[118,155]$, including visible radiation. These experiments used small pump currents typically below $1 \mu \mathrm{A}$, meaning that either a low number of electrons or only single electrons are simultaneously present in the crystal. Naturally, such results are well-described by single-electron models [119, 156, 156-158] which neglect any feedback of the generated light on the electrons. However, when the number of electrons is increased to enhance the amount of generated radiation, single electron-models fail to describe feedback. Therefore, no electron bunching causing stimulated emission and laser oscillation can be predicted, even for high electron currents.

So far feedback from Čerenkov radiation in photonic crystal modes on electrons has typically been described using a one-dimensional approach [159]. In the model, the electromagnetic field has been approximated as a superposition of at most up to three plane waves with different phase velocities propagating in the same direction and the slowly varying envelope approximation is applied [159]. While such modeling allows the description 
of exponential growth and the emergence of gain saturation, the according dynamic development of electron bunching has never been presented. Fundamentally, the restriction to three plane waves also fails to correctly describe the growth of modes inside photonic crystals for three reasons. First, a one-dimensional scheme neglects the transverse variation of the electromagnetic field which alters the gain. Second, the scheme fails to describe that the photonic crystal modes are composed of an infinite number of spatial harmonics, each with its own wave number, in order to fulfill all the boundary conditions of a photonic crystal. Third, the slowly varying envelope approximation becomes invalid at the limit of small group velocities [160], which are routinely experienced at the edge of a spatial harmonic, i.e., $k_{\xi}=m \pi / a_{\xi}$ (see Fig. 2.2). This invalidity is a fundamental limitation because the linear theory (see section 2.6) predicts a diverging gain at exactly these frequencies (see eq. (2.47)). A diverging gain cannot be realistic because such growth would, eventually, be in contradiction with the law of energy conservation. While interesting work has been published for a single set of parameters using also a three-dimensional model [161], this description of stimulated emission and laser oscillation generated by free electrons traveling through photonic crystals remains far from being complete. Therefore, previous work is incapable of correctly predicting the full spatio-temporal dynamics for pFELs.

In this chapter we present a complete and detailed investigation of a pFEL based on three-dimensional PIC calculations. As pointed out in chapter 2.7 such calculations are direct numerical solutions of the coupled nonlinear differential equations, with all transverse variations included, all boundary conditions fulfilled, and in which the slowly varying envelope approximation is dropped. To reduce the complexity of the study we limit ourselves to a single electron beam in this chapter. An extension to multiple beams is given in the next chapter. In section 3.1 we present the general working principle for pFELs. In section 3.2 we apply the working principle, especially velocity-matching, to predict the operating frequencies of pFELs. To provide for the first time a comprehensive investigation of the oscillation of a pFEL, we analyze the prototype of a basic pFEL pumped by a single electron beam by using a PIC method (see section 3.3). First, we study in great detail the start-up dynamics of the electromagnetic field and the electron beam dynamics for a typical electron velocity. Then, we investigate the laser threshold, steady-state output power and the frequency tuning upon change of the electron velocity. While the PIC calculations on which these results are based offer a great amount of insight into the detailed dynamical behavior of the laser, these calculations are also extremely time-consuming. Using simplified theories, such as linear gain theories, might be much faster, but the inherent approximations might lead to deviations. The magnitude of these potential deviations is largely unknown. In fact, for the pFEL, simplified theories have 
never been validated versus nonlinear PIC calculations. Such a comparison is presented in section 3.4. Section 3.5 summarizes the main conclusion from these investigations.

\subsection{Working principle of a pFEL}

To describe the working principle, we combine theoretical work on photonic crystals with Čerenkov emission and slow-wave FEL theory. We formulate the principle using terms such as spontaneous and stimulated emission to underline the close analogy to other types of lasers. The gain in a pFEL is provided by electrons which stream through a photonic crystal. When a single electron enters the crystal it induces dipoles in the material of the photonic crystal due to its space-charge field. This is similar to the Čerenkov effect in homogeneous, isotropic dielectrics (see section 2.2). As the electron propagates, the induced dipoles show a time dependent strength and orientation such that the positive charge of the dipoles remain oriented towards the passing electron. Due to the dipoles time dependence, they emit light in the form of wavelets. The total emission induced by a single electron is formed by the interference of all these wavelets, just as in a homogeneous isotropic dielectric. However, unlike the emission in these materials, the periodic environment in a photonic crystal significantly modifies the total emission for even a single electron. In a simple dielectric the wavelet propagation is described by the evolution of a spherical wave. In contrast, for photonic crystals each propagation direction may possess a different phase and group velocity. As a consequence Čerenkov emission inside photonic crystals reveals unique properties, such as the lack of a velocity threshold and an optimized emission for mode frequencies that are velocity-matched in a low spatial harmonic $[118,119,125,156,157]$.

The reason why emission into velocity-matched frequencies is maximized can be illustrated by looking at a particular example. Assume a specific photonic crystal set up from an array of infinitely long metallic rods in vacuum spaced a distance $a$ from each other. A single, passing electron induces a time-dependent dipole into each metallic rod. This is because electrons in the metal are quasi free to be displaced by the external field of the passing free electron. Thus, each rod emits a single wavelet. From each rod the emitted wavelet propagates in all directions. While the detailed evolution of the wavelet is complex, assume that a part of the wavelet propagates with a matched phase velocity along the electron propagation direction. This part of the emitted wavelet arrives at the next rods simultaneously with the electron and the next wavelet emissions are all temporally synchronized with the first wavelet. This leads to a constructive interference between all generated wavelets. At frequencies where no such velocity-matching exists, the emission 
from many rods destructively interferes. This indicates that a maximum amount of radiation should be emitted into a velocity-matched frequency of a certain photonic crystal mode. When comparing the emission strength for different modes one should take into account that the spatial harmonic amplitude usually decays with an increasing order of the spatial harmonic, $m$. Thus, one would expect that velocity-matching at low spatial harmonics leads to a stronger emission, compared to matching at higher spatial harmonics. Indeed, this has been calculated by Kremers [125], using a complex mathematical formalism. In conclusion, the emission from a single electron in a photonic crystal is enhanced at the velocity-matched frequencies of the modes.

So far the described emission from single electrons has been presented in the framework of a entirely deterministic classical theory. As a result the Čerenkov emission is coherent, as the emission process and the initial velocity and position of the electron is completely deterministic. However, electron velocity and position are always compromised by noise. The most important noise effects for any slow-wave FEL are shot noise and velocity noise. Shot noise is due to the discrete nature of electrons and the process to generate free electrons. For example, the electron emission of thermionic cathodes usually employed in slow-wave FELs is a statistic process [89, 162]. Velocity noise arises due to the Maxwellian velocity distribution of emitted electrons from a hot cathode $[89,163]$. Thus, the electron position and momentum are not exactly determined. It is this uncertainty in the electron position that makes the time of emission fundamentally unpredictable. This is a central characteristic of spontaneous, i.e., incoherent emission. In this perspective, the single electron emission in a photonic crystal can be characterized as spontaneous emission.

In a pFEL instead of a single electron many electrons are simultaneously present in the photonic crystal, giving rise to a further effect that influences the emission. While the emission of an individual electron generated at each rod still adds constructively when neglecting noise effects, this is not necessarily the case with the emission from different electrons. The total emission is given by the total wavelet interference emitted by all electrons. Therefore, the interference is also dependent on the phase between two wavelets emitted from different electrons, i.e., it depends on the relative position of the electrons in the crystal. For a continuous stream of electrons, electrons are roughly homogeneously distributed throughout the entire crystal. Such approximately homogeneous electron beam leads to an overall approximately destructive interference. Only the remaining deviations from a homogeneous distribution, which are weak and random, generates random wavelets, i.e., incoherent emission. We designate this emission process as spontaneous emission owing to its random, i.e., incoherent emission character.

For achieving coherent emission from the electron beam a proper correlation of the dis- 
tance between electrons inside the beam is required, which lets the emitted wavelets from all electrons constructively interfere. Ideally the phase difference between each wavelet should be a multiple of $2 \pi$ (including zero). This can be realized when the spacing between the electrons is a multiple of a wavelength of the electromagnetic field of the specific photonic crystal mode to be excited.

To explain the origin of coherent emission in more detail assume again a continuous, homogeneous, stream of electrons propagating through the photonic crystal. As was mentioned, to achieve coherent emission, the electron spacing needs to be re-arranged to attain the same periodicity as the field that is to be amplified. This rearrangement then sets a phase difference between wavelets which is a multiple of $2 \pi$. This translates into a electron distance of which is a multiple of $\lambda_{z, m}=2 \pi / k_{z, m}$, where $k_{z, m}$ is a single velocity-matched wave number corresponding to a low spatial harmonic of a certain photonic crystal mode. However, the electron spacing in a general electron beam is initially homogeneous with random components. Hence, only spontaneous radiation is emitted. The random components may actually generate a randomly phased light field in quite a number of velocity-matched modes. For achieving coherent emission, at least one of these matched modes needs to possess a longitudinal electric field component $E_{z}$. It is this field component which can impose a net force on the electron beam, thereby leading to the desired re-arrangement of the electron positions. In particular, if velocity-matching of such a specific photonic crystal mode occurs at a low spatial harmonic which provides a $E_{z}$ field component, a strong field interacts with the electrons throughout the entire photonic crystal. When the electron beam enters the crystal, this will initially induce a small, spatially periodic modulation of the electron velocities. Upon further beam propagation this is converted into a density modulation at the wavelength of the velocity matched spatial harmonic $\lambda_{z, m}$, thus, electron bunching. The bunching leads to enhanced emission into the original field of the mode at a phase that matches that of the originally present phase. Under these conditions the interference improves and an oscillating field builds up together with an enhanced bunching. This mutual generation of a properly phased bunching by the field and a properly phased field by the bunching allows the field in the photonic crystal mode to grow exponentially with the crystal length. Just as in other lasers, it does not actually matter which absolute phase the initial spontaneous emission possess, as the described process of exponential field growth generates an amplified wave with the same phase. This is why the amplification can also be named stimulated, in analogy with other types of lasers.

The described mechanism may be used to amplify an external signal in a single pass. If the photonic crystal is placed inside a resonator the amplification repeats each round-trip. 
If the gain in the photonic crystal section exceeds the losses of the resonator, the field in the photonic crystal mode should start to grow from initial spontaneous emission inside the resonator. Eventually, a strictly periodic electric field should oscillate in the resonator. It is expected that, finally, the gain reduces, which is called gain saturation, when the power in the oscillating photonic crystal mode exceeds a certain value. Similar to other FELs, gain saturation should be related to a change of the electron velocity dynamics as follows. When electrons have emitted a significant amount of kinetic energy in the form of radiation and their velocity is strongly reduced, the electromagnetic field starts to overtake the electrons $[76,89,164]$. If the electromagnetic field slips by more than half a period over the electrons and thereby switches its polarity, the electrons become re-accelerated by the electromagnetic field. Such electrons absorb light, instead of amplifying it. This re-absorption reduces the net gain (saturates the gain) until it becomes balanced with the losses. Thus, gain saturation leads to a steady-state emission with constant output power.

So far we have not included the Coulomb interaction between the electrons in the beam. Including this interaction would qualitatively change the description in two ways. First, Coulomb repulsion obviously limits the length to which electron bunches can be compressed. This limits the ability of all electrons to emit in phase. Thereby, it forms a limit for the maximum available gain. Second, as pointed out in section 2.5, the Coulomb interaction is responsible for a collective motion in the form of fast and slow space-charge waves. Only the slow space-charge wave is capable to transfer kinetic energy from the electron beam into the build-up of an electromagnetic field. The effective velocity of that space-charge wave becomes lower with increasing beam current. Hence, the frequency of the amplified electromagnetic wave, should depend on the beam current as well and not only on the initial kinetic electron energy.

\section{$3.2 \quad$ pFEL model}

To investigate the predictions from the last section and provide a solid theoretical background for subsequent experimental realizations we study in the following the properties of a specific pFEL in greater detail. We chose a specific design of a photonic crystal for operation in the microwave region. The setup of this pFEL is depicted in Fig. 3.1. Gain is provided by streaming a single electron beam through a section of a metallic photonic crystal slab. The length of this section is 20 unit cells. The unit cell is shown as an inset in Fig. 3.2. A rectangular metal waveguide, which is $w=8.4 \mathrm{~mm}$ wide and $h=8 \mathrm{~mm}$ high, encloses the photonic crystal slab. The photonic crystal slab consists of a rectangular 


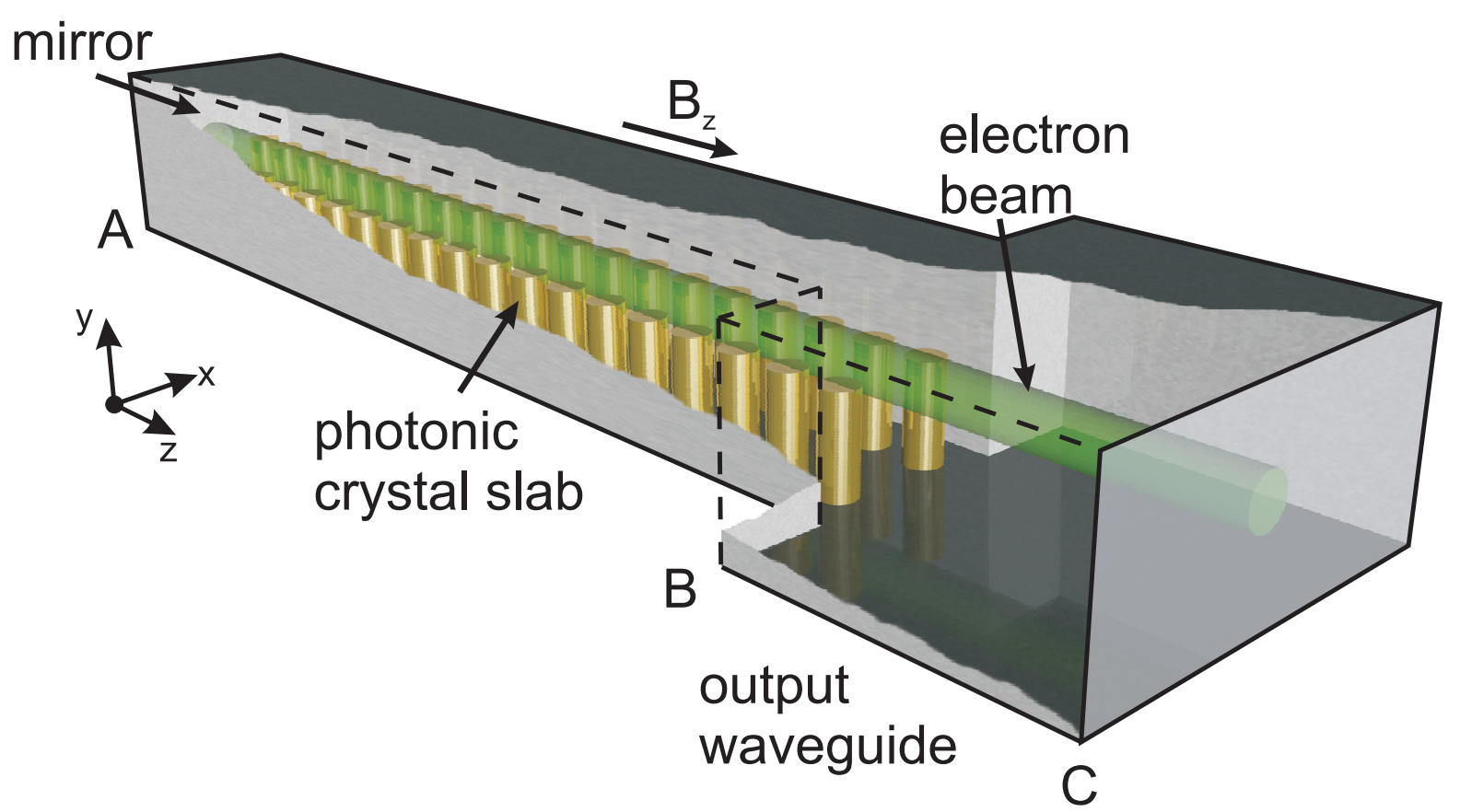

Figure 3.1: Schematic overview of a photonic free-electron laser pumped by a single electron beam. The electrons enter at plane A into a photonic crystal slab that provide gain. The gain section of 20 unit cells is placed inside a resonator, formed by a fully reflecting metallic mirror at $\mathrm{A}$ and a partially reflecting plane $\mathrm{B}$. In plane $\mathrm{B}$, the photonic crystal slab is terminated and the waveguide is widened to a bigger cross section. In plane $\mathrm{C}$, the so-called output port, the generated electromagnetic wave is sampled in time and decomposed into the modes of the output port. Throughout its entire propagation length the electron beam is guided by a static, homogeneous, longitudinal magnetic field, $B_{z}=0.5 \mathrm{~T}$. 
lattice of metallic rods. In the longitudinal $(z)$ direction the spacing between the center of the rods is $a_{z}=2.5 \mathrm{~mm}$ and in the transverse $(x)$ direction it is $a_{x}=4.2 \mathrm{~mm}$. The radius of the rods is $r_{p}=0.75 \mathrm{~mm}$ and the rod height is $h_{p}=4 \mathrm{~mm}$.

For the electron beam we choose typical parameters from other slow-wave FELs that operate at microwave frequencies [64]. The diameter of the cylindrical electron beam is $2 \mathrm{~mm}$ and its beam current is $1 \mathrm{~A}$. We restrict to rather low energy electrons of about $E_{e l}=$ $15 \mathrm{keV}$, thus $v=0.243 c$, which would be of interest for an overall compact size, but which requires very slow waves inside the photonic crystal. To investigate the frequency tuning of the pFEL the electron energy is varied. We consider energies between $10 \mathrm{keV}$ to $15 \mathrm{keV}$, corresponding to electron velocities between $v=0.199 c$ and $v=0.243 c$. Further, we assume that the initial velocity spread can be neglected compared to the velocity changes that occur during interaction. To reduce the divergence by Coulomb repulsion between the electrons, the electrons are guided throughout the laser by a static, homogeneous, longitudinal magnetic field of $B_{z}=0.5 \mathrm{~T}$.

The gain section is located inside a resonator which is set up by a fully reflecting mirror at plane $\mathrm{A}$ and a partially reflection at plane $\mathrm{B}$. The latter reflection originates from the termination of the photonic crystal slab into a waveguide. To illustrate this, we recall that a certain effective dielectric constant can be associated to the photonic crystal slab. Then it is clear that at $\mathrm{B}$ the dielectric constant changes from the effective index of the slab to the effective index of the waveguide, resulting in a partial reflection from that interface. In appendix A the resulting partial reflection at B is calculated to be about $95 \%$. The width of the output waveguide is chosen such that for the predicted laser frequencies, only the lowest order waveguide mode, $\mathrm{TE}_{10}$, propagates. All higher order modes have a cut-off frequency above $17.8 \mathrm{GHz}$ and cannot propagate in the output waveguide. Therefore, only emission in the lowest order waveguide mode is expected to arrive at plane $\mathrm{C}$, the so-called output port. In order to later analyze the output generated by the laser, we terminate the output waveguide with a non-reflective boundary in the output port. There the electromagnetic field pattern is sampled versus time and the electromagnetic field pattern is also decomposed into the eigenmodes of the output waveguide but, as higher order modes are evanescent, they contain only a noise-like signal with a low power level. The length of the output waveguide of $13.75 \mathrm{~mm}$ is a compromise between minimizing the size of the modeled volume and preventing out-coupling via evanescent waves to the output port.

According to the working principle discussed in the last section, it should be possible to estimate the operating frequency of the pFEL by searching for velocity-matching between the slow space-charge wave and the photonic crystal modes. To find the velocity-matched 


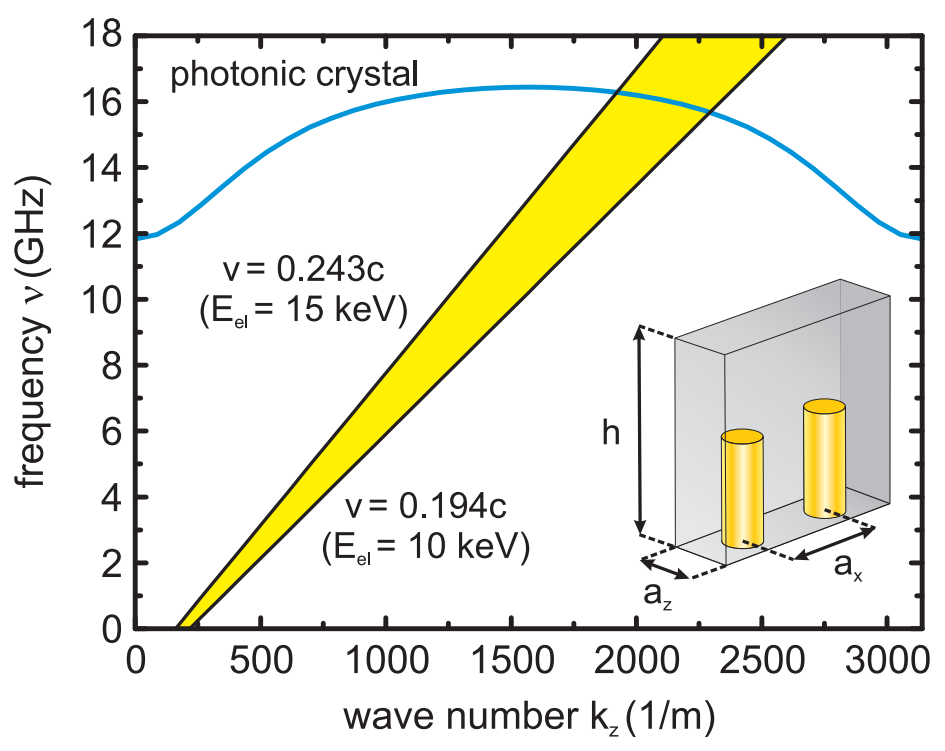

Figure 3.2: Photonic band structure of the lowest frequency photonic crystal mode with $E_{z} \neq 0$ and range of slow space-charge waves for electrons with velocities from $0.194 \mathrm{c}$ to 0.243c. Inset: Unit cell of the photonic crystal slab defining the geometry parameters, $a_{x}=4.2 \mathrm{~mm}, a_{z}=2.5 \mathrm{~mm}, h=8 \mathrm{~mm}$ and $w=8.4 \mathrm{~mm}$. The height of the rods is $h_{p}=4 \mathrm{~mm}$ and the radius of the rods is $r_{p}=0.75 \mathrm{~mm}$.

frequencies we calculate the dispersion ( $\nu$ versus $k_{z}$ ) of both waves. At the intersection points of the dispersion curves, the phase velocities $\left(v_{p h}=2 \pi \nu / k_{z}\right)$ of both waves match. The dispersion of the slow space-charge wave is given by eq. (2.33). The dispersion of the photonic crystal modes has to be numerically calculated for each specific crystal geometry, due to the complexity of the boundary conditions in photonic crystals. For simplicity, all metal parts are treated as perfect electric conductors which is well justified in the microwave range [165]. We use an iterative eigenmode solver [141] with periodic boundary conditions along the $z$-direction, i.e., we solve for eigenmodes of an infinitely long photonic crystal slab with a specific phase advance per unit cell. Essentially, this means that one specifies a certain wave number $k_{z, 0}$. The iterative solver then searches for a specified number of eigenfrequencies that solve the master equation (2.12) for the selected wave number $k_{z, 0}$. These frequencies correspond to the eigenmodes of the crystal. By varying $k_{z, 0}$ throughout the range of the $0^{\text {th }}$ spatial harmonic $(m=0)$, i.e., $-\pi / a_{z}<$ $k_{z, 0}<\pi / a_{z}$, we find the dispersion of the photonic crystal modes. In addition, the solver also computes the field distribution of the modes throughout the unit cell. Note that, due to the use of periodic boundary conditions also all wave numbers of the higher spatial harmonics, i.e., $k_{z}=k_{z, 0}+2 m \pi / a_{z}$, are contained in the electromagnetic field distribution of the eigenmodes of the structure. Recall, as stated by eq. (2.23), that the 
field of each photonic crystal mode comprises an infinite number of spatial harmonics. Here, we restrict the eigenmode search by implementing symmetry conditions in order to find only the modes that possess a non-zero longitudinal electric field component at the center of the waveguide. Only such modes can induce an electron bunching and thus become amplified. A more detailed explanation of such calculations can also be found elsewhere [166].

For the photonic crystal slab we study here, Fig. 3.2 shows the results of these calculations. The curved trace gives the dispersion of the lowest frequency photonic crystal mode found. The straight lines give the dispersion for the slow-space charge wave at the highest and lowest electron velocity. To indicate intermediate velocities the range in between is colored. It can be seen that, depending on the specific choice of the electron velocity, velocity matching occurs for frequencies between $15.6 \mathrm{GHz}$ and $16.3 \mathrm{GHz}$. Next to the shown photonic crystal mode also other modes exist. These modes all appear above $18 \mathrm{GHz}$. Of course, these modes are also velocity-matched, but matching occurs at much higher wave numbers, i.e., in higher spatial harmonics. The Fourier amplitude of the matched longitudinal $E_{z}$ field component of these modes is weaker. According to eqs. (2.46) to (2.49) this should lead to a lower small-signal gain. Indeed, it turns out that these modes do not start-up in the laser and we do not discuss them any further in this chapter.

For comparison with later results, we also predict the expected electron bunching period, from the velocity-matching. To the predicted operating frequency range corresponds a ranges of velocity-matched wave numbers $k_{z, P h C}$. From Fig. 3.2a this range of wave numbers is found to extend from $1533 \mathrm{~m}^{-1}$ to $1830 \mathrm{~m}^{-1}$. From section 2.5 we expect that this is the wave number of the slow space-charge wave excited on the electron beam. Thus, the expected electron bunching period $\lambda_{e l}$ can be deduced from these velocity-matched wave numbers $k_{z, P h C}$ via

$$
\lambda_{e l}=\frac{2 \pi}{k_{z, P h C}} .
$$

The equation yields values between $3.4 \mathrm{~mm}$ and $4.1 \mathrm{~mm}$ for the given frequency range. Note that, this wavelength range is also equal to the velocity-matched wavelength $\lambda_{P h C}$ of the photonic crystal mode. 


\subsection{Particle-in-cell calculations}

As is pointed out in the introduction, analytical studies fail to describe the full pFEL interaction. One of the reasons is that one-dimensional studies neglect the full properties of a photonic crystal [159]. The only three-dimensional study concerning amplification in a photonic crystal, does not give results for the dynamical evolution of the electromagnetic wave, the electron beam dynamics and the frequency tuning of the laser. Thus, it can only partly verify the above discussed dynamical growth from noise towards gain saturation [161]. To investigate the oscillation of a pFEL for the first time comprehensively and to provide a solid theoretical background for subsequent experimental realizations, we study the specific laser model shown in Fig. 3.1 via extensive numerical PIC modeling [141]. The PIC method gives a self-consistent solution of the coupled Maxwell and Newton-Lorentz equations that describe the interaction in pFELs. However, this comes at the expense of long computation times. Typical computation times for a single set of parameters are in the order of several days on a high end, multi-core PC.

To reduce the computational demands to a level that can be easily processed by affordable multi-core workstations, we perform four simplifications of the model. First, as is common for PIC methods, we use super-particles, as described in section 2.7. For our case, we choose the super-particles to have a charge and mass of about 16,200 electrons. This reduces the average number of charged particles simultaneously present in the laser from 6 billion electrons to 350,000 super-particles. This means that, at each time step $(\Delta t=320 \mathrm{fs}), 121$ of such super-particles are injected into the laser at plane A to model a beam current of $1 \mathrm{~A}$. Second, we do not store the output data - the electromagnetic field distribution and all particle positions - each time step. The electromagnetic field is only sampled at the output port every $9.7 \mathrm{ps}$, and all particle positions are stored throughout the entire modeled volume each nanosecond. Third, we limit the mesh resolution to 30 mesh cells per free-space wavelength, resulting in a total of 256,300 mesh cells which turns out to be a good compromise between computation time and numerical accuracy. Fourth, as indicated before, all metal parts are treated as perfect electric conductors. These measures allow the reduction of the computation time for a single set of parameters to about one day.

\subsubsection{Laser operation with fixed electron velocity}

For verifying the suggested behavior of the dynamical growth for the pFEL we first study the laser dynamics, the electron beam dynamics, and the laser threshold at a fixed electron velocity. As a typical example, we chose a mid-range beam energy of $12.5 \mathrm{keV}$ (Fig. 3.2), 
which corresponds to an electron velocity of $0.221 c$. For studying the dynamical growth, we chose, as initial conditions for the PIC calculation, to have no radiation field and particles are present inside the resonator. Thereafter, by increasing the number of superparticles from zero to 121 during the first 625 time steps, i.e., $0.2 \mathrm{~ns}$, the beam current is ramped up to a constant final value of $1 \mathrm{~A}$.

From the working principle of the pFEL we expect the laser start-up to be distinguished into three phases in time. First, spontaneous emission should be emitted. Second, an exponential increase of the output power should occur. Third and finally, the growth of the output power should stop due to gain saturation. Figure 3.3a shows the calculated temporal evolution of the power at the output port in a semi-log plot. To calculate the power, the electromagnetic field sampled at the output port is squared and time averaged with an averaging time of about 63 ps. Initially, the output power is weak and noisy as expected for spontaneous emission. After a few nanoseconds an exponential growth sets in, as indicated by the almost linear slope of the curve in the semi-log plot. This growth continues over many orders of magnitude. After about $50 \mathrm{~ns}$ the power growth reduces and, eventually, at about $500 \mathrm{~ns}$ a steady-state output power of $1.5 \mathrm{~kW}$ is reached. Thus, indeed the three phases of dynamical power growth, spontaneous emission, exponential growth and gain saturation can be recognized in the start-up of a pFEL.

To clarify the difference in emission during the different start-up phases of the laser, Fig. 3.3b-d, show the electric field including carrier frequency oscillations at three characteristic time windows during the growth. At an early time, around $t=6 \mathrm{~ns}$, according to spontaneous emission the electric field is extremely weak and the amplitude fluctuates (see Fig. 3.3b). Phase fluctuations in the oscillating electric field are also observed, e.g., at $t=5.7 \mathrm{~ns}$. Such fluctuations in an electromagnetic wave are characteristic for a random interference of wavelets, i.e., spontaneous emission. Figure 3.3c shows the electric field for a somewhat later time, at around $t=33 \mathrm{~ns}$. During this times exponential growth in the power evolution is observed and in Fig. 3.3c a regular oscillation without fluctuations is found. This is indeed confirming that a well phase-matched interference of wavelets occurs, which leads to amplification and is a signature of stimulated emission. Well into steady-state at about $t=204 \mathrm{~ns}$ the field is found to be constant (see Fig. 3.3d). The field oscillation is again regular without phase fluctuations, which corresponds to a single-frequency emission with a narrow spectral bandwidth.

In order to investigate how well-defined the frequency of this oscillation is, we look at the power spectrum of this oscillation. In principle, noise should impose a lower limit to the bandwidth of the laser. Figure 3.4 shows the calculated power spectrum, obtained via Fourier-analyzing the complete sampled signal at the output port. This means the 

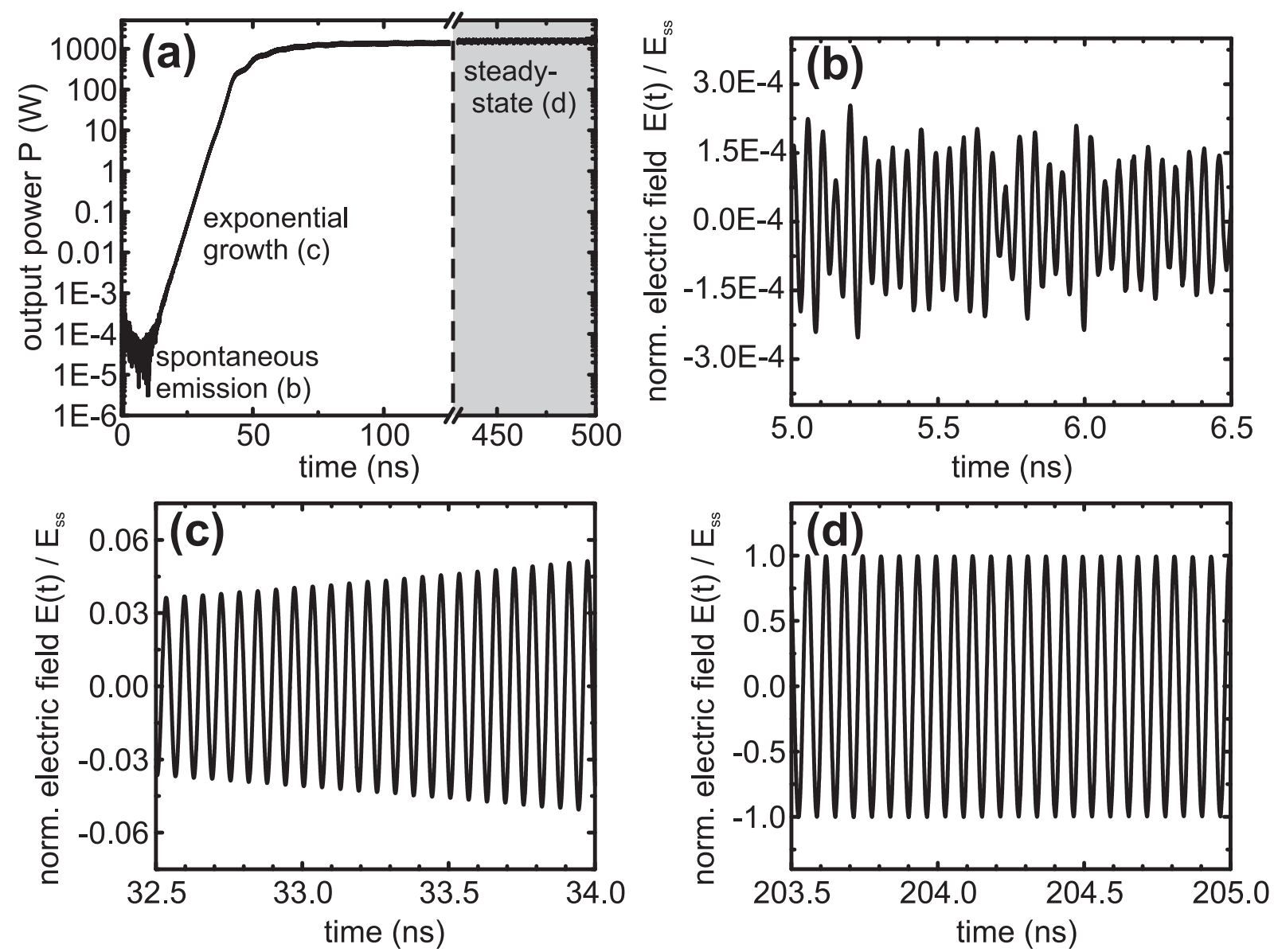

Figure 3.3: (a) Temporal evolution of the time averaged output power from a pFEL at the output port. The electron velocity is $0.221 \mathrm{c}$, thus $E_{e l}=12.5 \mathrm{keV}$. The power develops from low power spontaneous emission via an exponential growth to a constant steady-state output power $P_{s s}=1.5 \mathrm{~kW}$. (b) to (d) The oscillating electric field at three characteristic times during the growth. (b) During spontaneous emission at about $t=6 \mathrm{~ns}$ a fluctuating electric field amplitude and a fluctuating phase is found. (c) During exponential growth at about $t=33 \mathrm{~ns}$ a regular oscillation is found with a continuously growing amplitude. (d) Well into steady-state, at about $t=204 \mathrm{~ns}$, a single-frequency oscillation with a constant electric field amplitude is observed. 


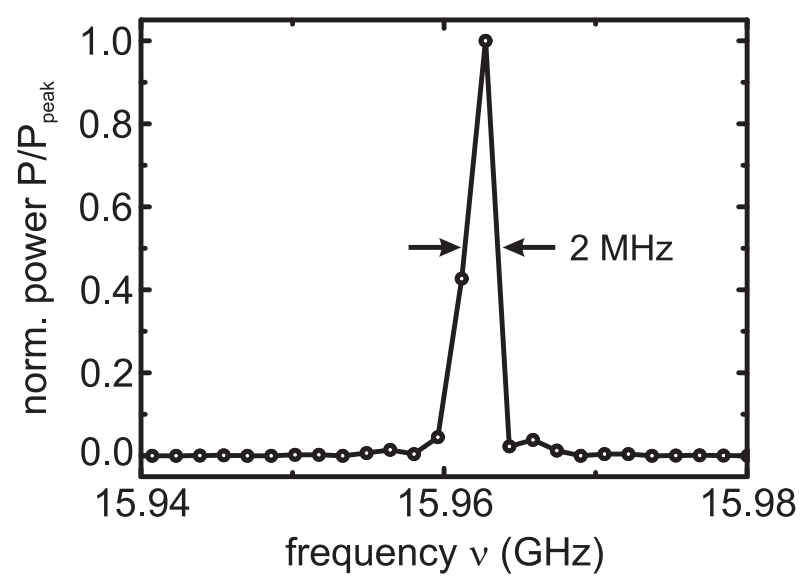

Figure 3.4: Power spectrum of the sampled electromagnetic wave at the output port.

signal from start-up at $t=0$, ns until $t=500$, ns where the calculations are stopped. The calculated data is shown as circles and we observe a single maximum in the spectrum. When using a linear interpolation between the individual data points to define the FWHM of this maximum one obtains a spectral bandwidth of about $2 \mathrm{MHz}$. Note that, due to the limited time over which numerical field data are available, $t_{\text {run }}=500 \mathrm{~ns}$, the Fourierlimited bandwidth is

$$
\Delta \nu=\frac{1.12}{t_{\text {run }}}=2.24 \mathrm{MHz}
$$

This limit is given by the FWHM of the Fourier-transform for a rectangular time window of $t_{\text {run }}=500 \mathrm{~ns}$. The good agreement with the bandwidth found in Fig. 3.4 shows that the observed oscillation bandwidth is currently limited by the time over which field data is available. Much longer calculation times would be required to identify physical contributions to fundamental bandwidth limit of the laser. In conclusion, we have found that, as expected, the laser start-up can be coarsely divided into three phases. The last of these phases is characterized by a single frequency emission with a constant power (steady-state). However, the detailed origin of gain saturation which determines the amount of steady-state output power cannot be deduced from the power evolution or frequency spectrum.

In order to investigate the physical origin of gain saturation we study the dynamics of the electron beam. We expect that, initially, a nearly homogeneous electron velocity distribution and space-charge distribution is found along the longitudinal $z$-direction . During exponential growth both distributions should be periodically modulated along $z$. The period length of both modulations should be equal to the electron bunching period $\lambda_{e l}$ 
and the velocity-matched wavelength $\lambda_{P h C}$ of the interacting photonic crystal mode. We further expect that, eventually, gain saturation should reveal itself by a strong deceleration of electrons and loss of bunching. In the following we investigate these predictions. We study the velocity of each super-particle and the charge density in the electron beam as a function of the longitudinal coordinate $z$ throughout the laser resonator. As is common, we chose to express the velocity distribution in terms of the electron energy, $E_{e l}$. As $\gamma$ is close to unity, for the low energy beams we consider here, the electron velocity $v$ is given by $E_{e l}=\frac{1}{2} m_{e l} v^{2}$. In order to take representative moments in time we select again the times $t=6 \mathrm{~ns}, t=33 \mathrm{~ns}$ and $t=204 \mathrm{~ns}$. These times correspond to spontaneous emission, exponential growth and steady-state, respectively.

Figure 3.5a depicts the energy of each super-particle versus the normalized longitudinal position $z / a_{z}$ at a time $t=6 \mathrm{~ns}$. At this moment the laser emits spontaneously, i.e., hardly any light is present inside the photonic crystal slab. One might expect that in this case the kinetic energy of the mono-energetic electron beam should remain constant upon propagation through the crystal. However, it can be seen that the initial electron energy of $12.5 \mathrm{keV}$ reduces by about $250 \mathrm{eV}$ when the super-particles are injected into the resonator at $z / a_{z}=0$, which is plane $\mathrm{A}$ in Fig. 3.1. Further, the beam acquires an energy spread of about $160 \mathrm{eV}$ and the electron energy displays a small periodic modulation with a period length of about $2.5 \mathrm{~mm}$. Finally, when the beam enters the output waveguide at $z / a_{z}=20$ (plane B in Fig. 3.1) the electron energy decreases further and the periodic modulation disappears.

These observations can be addressed to the following effect. The electrostatic field generated by the electrons must be perpendicular to all metallic surfaces of the resonator, making these equi-potential surfaces. Thus, the equi-potential surfaces of the electrostatic field have to be the waveguide walls, the metallic mirror and the metallic rods [167]. In contrast to this, if a single electron would propagate in free-space, their electrostatic field would decrease with $1 / r^{2}$ exhibiting spherical equi-potential surfaces. Bringing a metallic surface with a deviating shape or curvature into the field of a free electron implies that charge in the metal surface has to become displaced in order to establish a zero electric field component tangential to the metal surface. However, such electron displacement means that the beam electrons acquire potential energy [168]. Due to energy conservation, the total energy of the beam must remain constant which accordingly reduces the kinetic energy of the beam electrons. The amount of potential energy taken from the kinetic energy is dependent on the distance between the individual beam electron and the surrounding metal surfaces. For a bigger distance between free electrons and metallic object a larger build-up of potential energy is found [168]. 

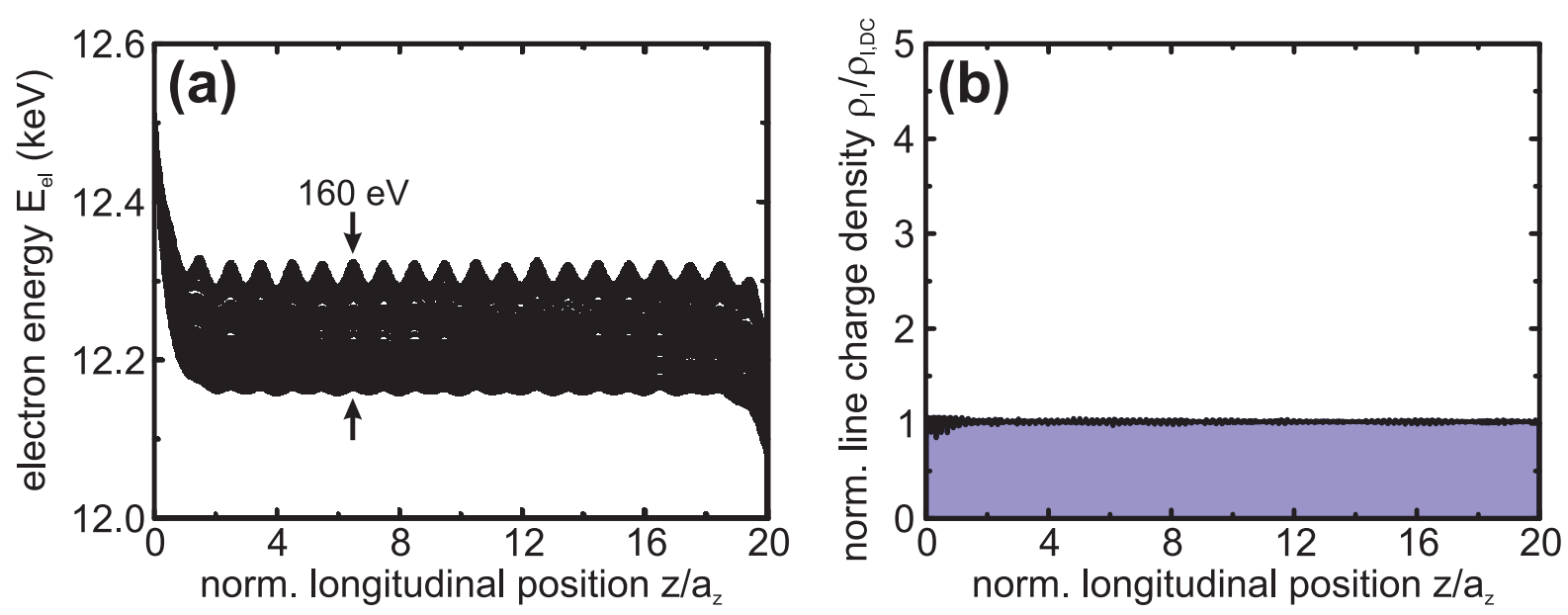

Figure 3.5: (a) Electron energy versus normalized $z$-position at $t=6$ ns. The injected electrons have a velocity of $v=0.221 c$, which corresponds to a beam energy of $E_{e l}=$ $12.25 \mathrm{keV}$. (b) Normalized line charge density $\rho_{l} / \rho_{l, D C}$ versus normalized $z$-position at $t=6 \mathrm{~ns}$.

Now, let us show how this effect does explain the observed electron energy changes in our model. Electrons are injected into the photonic crystal slab from a source located on the surface of the metallic mirror. When injected into the metal waveguide the electrons lose kinetic energy as described above. This leads to the immediate deceleration of electrons at the very beginning of the photonic crystal slab $z / a_{z}<1$. As the amount of potential energy build-up and deceleration dependents on the distance to the waveguide walls, the reduction in kinetic energy is dependent on the transverse electron position $(x, y)$. In the projection onto the $z$-axis this leads to the observed energy spread of about $160 \mathrm{eV}$, see Fig. 3.5a. Also, the periodic modulation of kinetic energy throughout the resonator can be attributed to the build-up and release of potential energy. When the electrons pass by the rods, the distance to the surface of the rods increases and decreases periodically. Indeed, the modulation in Fig. 3.5a has a period length of $2.5 \mathrm{~mm}$ which is equal to the longitudinal spacing between the center of the rods. The eventual decrease in kinetic energy at $z / a_{z}=20$ is due to the wider width of the output waveguide which leads to a larger potential energy of the electrons. After leaving the waveguide at plane C, (not shown in Fig. 3.5a) the velocity is found to increase back to its initial value of $E_{e l}=12.5 \mathrm{keV}$. Let us discuss the impact of these effects on the operation of a pFEL. In a pFEL the electron velocity determines the frequency where amplification takes place. We conclude, that due to the build-up of potential energy the range of velocity-matched frequencies should be slightly broadened and shifted. According to Fig. 3.2, the frequency should shift by a few $\mathrm{MHz}$ compared to what would be expected with the nominal velocity and zero velocity spread. 
While we expected a constant electron energy throughout the photonic crystal slab at $t=6 \mathrm{~ns}$, we observed several effects caused by the metallic boundaries. Let us now study whether further deviations from our expectation are also found for the space-charge density. We again expect that no modulation should occur for the space-charge density as a function of $z$, because hardly any radiation is present in the crystal at the time $t=6 \mathrm{~ns}$ and because a continuous stream of electrons is injected. For comparison between the calculated results and this expectation we compute the normalized projection of the charge density onto the $z$-axis from Fig. 3.5a as follows. We generate a histogram for the $z$ positions of the particles (bin size $0.1 \mathrm{~mm}$ ) and multiply it with the super-particle charge of $q_{s}=2.6 \mathrm{fC}$ to get the total charge in a bin. To retrieve the line charge density we divide it by the bin size. For normalization we calculate the DC line charge density inside the resonator by using:

$$
\rho_{l, D C}=\frac{\int_{S} J \mathrm{~d} S}{v}=I_{0} \sqrt{\frac{m_{e l}}{2 E_{e l}}}=0.152 \frac{\mathrm{nC}}{\mathrm{m}} .
$$

Here, $S$ is the beam cross section area. For the electron energy we use the mean kinetic energy value of super-particles inside the slab of $E_{e l}=12.25 \mathrm{keV}$, which is determined from Fig. 3.5a. The result is depicted in Fig. 3.5b.

When inspecting Fig. 3.5b we observe that, the line charge density is hardly modulated at $t=6 \mathrm{~ns}$, except for a very small modulation for $z / a_{z}<1$. The latter can be attributed to the injection dynamics described above. However, this modulation is quickly damped out and it can be concluded that spontaneous emission has no notable influence on the charge-density inside the slab at $t=6 \mathrm{~ns}$. While we observe here that spontaneous emission initially has no impact on the electron beam, we found previously that at later times a single mode grows exponentially. This implies that by then the longitudinal field of the mode should have re-arranged the velocity and space-charge distributions. More specifically, the period length of this modulation is predicted by eq. (3.1) to be $\lambda_{e l}=3.78 \mathrm{~mm}$ for the chosen electron velocity. To inspect the electron beam at a time during the exponential growth Fig. 3.6 shows the corresponding energy of the superparticles and space-charge density versus $z$ at $t=33 \mathrm{~ns}$. The initial kinetic energy of the electrons inside the slab of $E_{e l}=12.25 \mathrm{keV}$ is indicated as a red line in Fig. 3.6a. It can be seen that a clear periodic modulation along the $z$-direction is now present. The amplitude of the modulation of about $0.5 \mathrm{keV}$ is now much stronger than the effect of potential energy build-up (see Fig. 3.5a). Also the line-charge density in Fig. 3.6b shows such periodic modulation. Thus, as expected during exponential growth electron bunching is observed. The period length of this bunching and the energy modulation is found to 

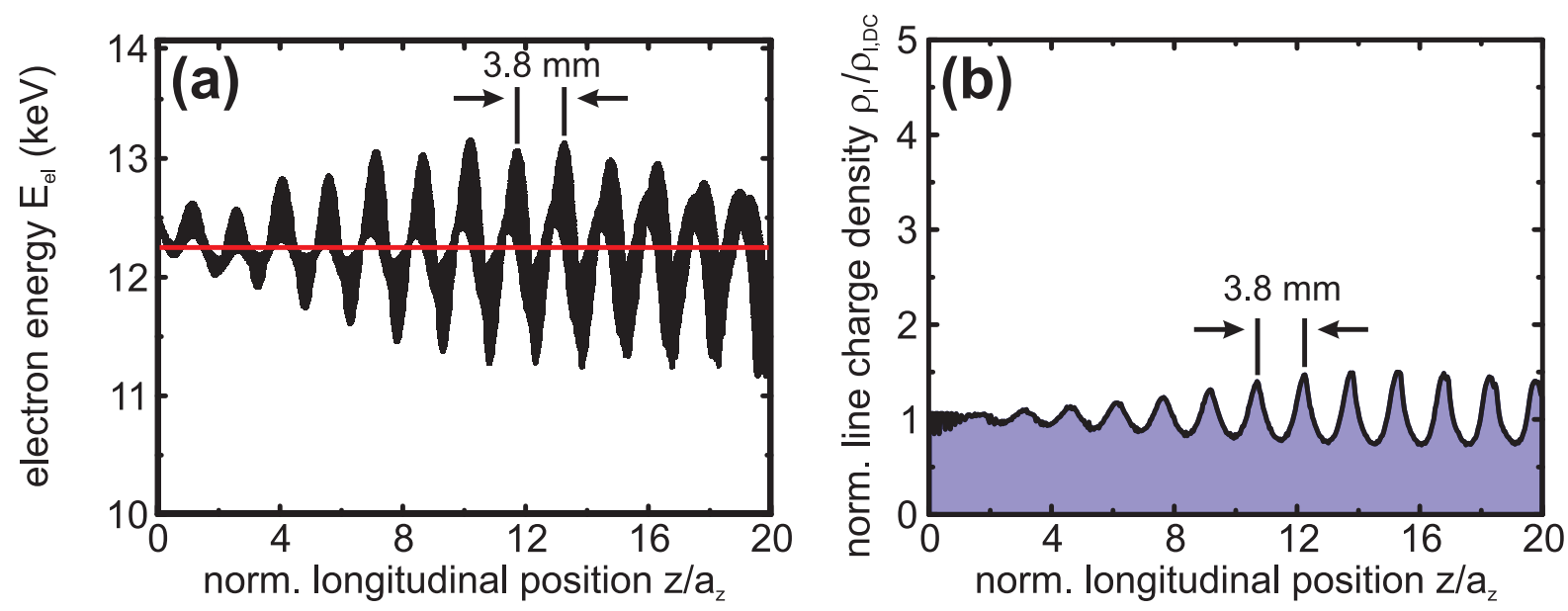

Figure 3.6: (a) Electron energy versus normalized $z$-position at $t=33 \mathrm{~ns}$ compared to the initial electron energy inside the slab of $E_{e l}=12.25 \mathrm{keV}$. (b) Normalized line charge density $\rho_{l} / \rho_{l, D C}$ versus normalized $z$-position at $t=33 \mathrm{~ns}$.

be $\lambda_{e l}=3.80 \mathrm{~mm}$ and agrees very well with the predicted period of $\lambda_{e l}=3.78 \mathrm{~mm}$. In addition, in both figures the amplitude of the modulation is more pronounced towards the output end of the laser resonator $\left(z / a_{z}>8\right)$. The development of bunching on the injected continuous stream of electrons requires a certain time interval, which is seen in Fig. 3.6b as a certain distance the electrons have to travel down the resonator.

To discuss the strength of the energy modulation, it is expected that during the exponential growth regime the total amount of kinetic energy transferred from the electrons to the electromagnetic field should be negligible when compared to the total beam energy [89]. From Fig. 3.6a we observe an approximately symmetric modulation around the initial kinetic energy for $z / a_{z}<12$, indicating that the amount of electrons that have been accelerated is almost the same as those that have been decelerated. Above this position a slight asymmetry is found with respect to the injected electron energy. When computing the average energy of all electrons inside the photonic crystal slab we find $E_{e l}=12.135 \mathrm{keV}$. This is only $90 \mathrm{eV}$ or $0.7 \%$ less than the injected energy. Indeed, during exponential growth hardly any kinetic energy is emitted from the electron beam. At much later times during the growth this has to change as the emitted power is much higher than at $t=33 \mathrm{~ns}$, meaning that the electrons have to lose a significant amount of kinetic energy.

Much later, at about $t=50 \mathrm{~ns}$, the growth in output power decreases and the gain starts to saturate. Eventually, a constant output power is emitted when the laser has reached steady-state. By inspecting the super-particle behavior well into steady-state, for example at $t=204 \mathrm{~ns}$, we should be able to find the origin of the corresponding gain 
saturation. Figure 3.7a shows the kinetic energy of the electrons at this time compared to the initial kinetic energy of the electrons inside the crystal. First of all we observe that the beam has acquired a large maximum energy modulation of $10.5 \mathrm{keV}$ and a far larger amount of electrons have lost energy than have gained. This is expected for the high amount of power emitted during steady-state operation (about 8\% of the electron beam power). In Fig. 3.7a a regular spatial periodic modulation is still visible near the entrance range, from $z / a_{z}=0$ to around $z / a_{z}=3.2$, just as during exponential growth. However, the minima and maxima are much more pronounced than in Fig. 3.6a. For positions further downstream, the energy distribution becomes more complex. While the accelerated electrons still show distinct peaks of increased energy, the decelerated electrons cease showing such simple behavior. Starting at the position marked by the star in Fig. 3.7a, the initially straight low energy electron dips become bent. For the following low energy dip at $z / a_{z}=7$ a small curl has developed from the initial bend. This curl becomes more and more pronounced for consecutive low energy dips. Eventually, it becomes impossible to find separate low energy dips (see $z / a_{z}=10$, and onwards) and in this range the energy distribution becomes very complex.

To understand this complex energy distribution, we realize that most electrons are already strongly decelerated in the first part of the photonic crystal slab by the strong electromagnetic field present in the crystal at $t=204 \mathrm{~ns}$. For example, after a propagation distance of $z / a_{z}=4$ the lowest energy electrons have already lost a substantial part $(5.8 \mathrm{keV})$ of their initial kinetic energy which corresponds to a reduction of their initial velocity by $27 \%$. In contrast, the generated electromagnetic field still propagates with the same phase velocity. Consequently, the slowed-down electrons slip continuously backwards in relative spatial phase with respect to the electromagnetic field. Eventually, the sign of the electromagnetic field changes, meaning that the electrons are not decelerated anymore. Instead of amplifying radiation, these electrons then absorb radiation and re-gain energy, which shows as a formation of curls in Fig. 3.7a. Due to the absorption, this part of the electrons reduces the field growth until a steady-state with a certain maximum intracavity power is reached. A certain fraction of this power, here about $5 \%$, is coupled out and detected as continuous-wave laser output. Getting further insights into the gain saturation is difficult when only investigating the energy distribution, due to its complex shape above $z / a_{z}>12$.

To investigate gain saturation in more detail, we turn to discuss the corresponding line charge density (Fig. 3.7b). For $z / a_{z}<8$ we observe electron bunches spaced by about $3.80 \mathrm{~mm}$ which is about one velocity-matched wavelength. In contrast to exponential growth in the small-signal regime (Fig. 3.6b), the modulation amplitude is higher 

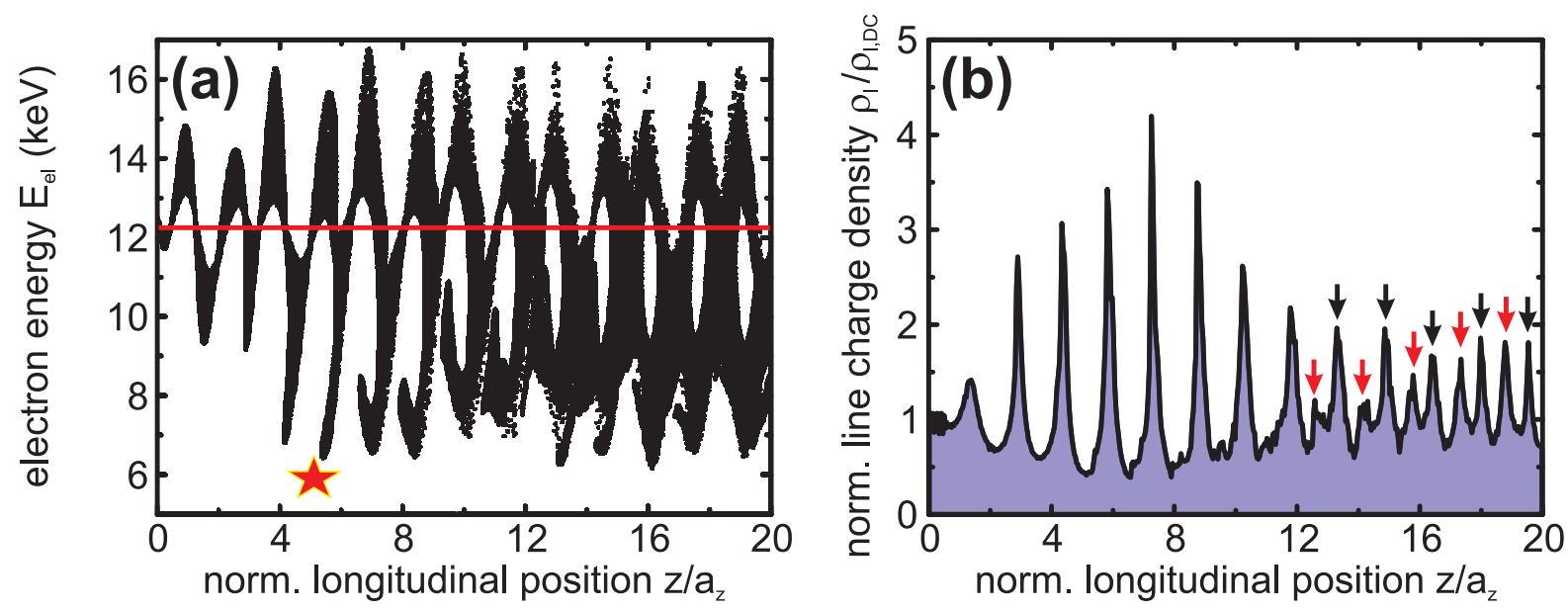

Figure 3.7: (a) Electron energy versus normalized $z$-position at $t=204 \mathrm{~ns}$ compared to the initial electron energy inside the crystal of $E_{e l}=12.25 \mathrm{keV}$. (b) Normalized line charge density $\rho_{l} / \rho_{l, D C}$ versus normalized $z$-position at $t=204 \mathrm{~ns}$ The red arrows mark the additional electron bunches that develop when the gain saturates, the black arrows mark the initial electron bunches.

and the bunches are narrower. This stronger bunching occurs because a much stronger bunching $E_{z}$ field is present at $t=204 \mathrm{~ns}$. However, beyond $z / a_{z} \approx 8$, the bunching decreases. Further, from about $z / a_{z}>12$, additional bunches grow between the initial bunch - marked in the figure by red and black arrows, respectively. Eventually, both types of bunches reach the same modulation amplitude. The spacing between two consecutive additional bunches and two consecutive initial bunches is again about $3.8 \mathrm{~mm}$. The spacing between an initial and an additional bunch eventually becomes about one half of the velocity-matched wavelength $\lambda_{P h C} / 2$ such that the amplifying and de-amplifying action of both types of bunches approximately cancels. This observation is in good agreement with the origin of saturation in other slow-wave FELs. An example is the traveling-wave tubes, where the evolution of a second set of electron bunches has also been reported at saturation [164].

So far we have investigated the growth and transition of the output power into steadystate along with the corresponding electron beam dynamics. These properties could be qualitatively explained within the framework of a simple working principle. Of course, such a description cannot predict a quantitative value for the threshold current and the steady-state power. For assessing the chances of an experimental verification it is particularly important to determine the expected threshold pump current $I_{t h}$ for a given pFEL setup. In the following, we present how to use an extrapolation for the determination of the threshold. This procedure is analogous to the procedure used to find the threshold in other types of lasers [169]. 


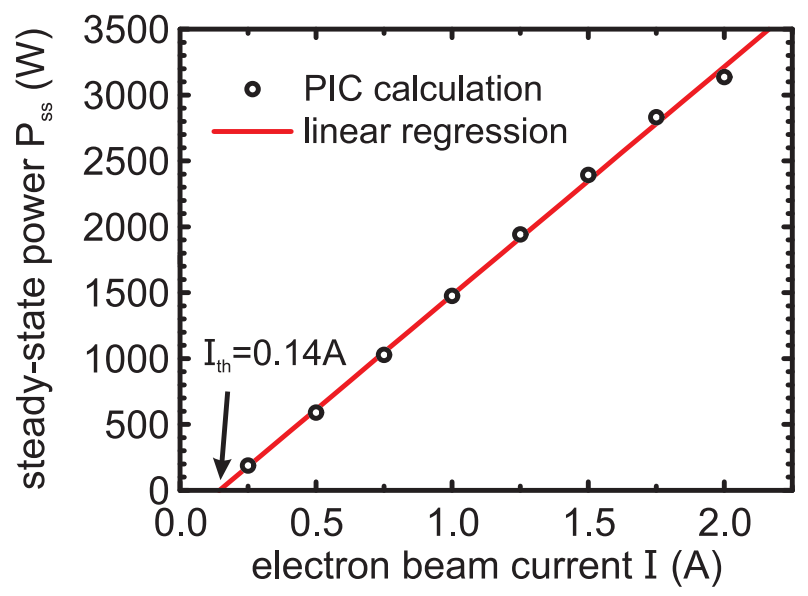

Figure 3.8: Steady-state power $P_{s s}$ versus electron beam current $I$.

Figure 3.8a shows the calculated steady-state output power $P_{s s}$ as obtained from a set of PIC calculations versus the beam current. Applying a linear regression to the calculated data points - depicted as a red line - reveals that the output power increases in a very good approximation linear with the pump current. The threshold current for the laser is then estimated by extrapolating the regression line to zero output power. We find a threshold current of about $I_{t h}=140 \mathrm{~mA}$ for the pFEL. When we compare this value to the electron current of $1 \mathrm{~A}$ we used throughout this chapter, which is actually motivated by experimental considerations (chapter 6), the threshold current seems to be readily available.

For completeness we note that some care has to be taken when using the PIC calculations for extrapolating a threshold current. Namely, as has been discussed in section 3.1, the operation frequency of the laser depends not only on the kinetic energy, but also on the beam current. Furthermore, as will be discussed in the following section, changing the laser frequency also varies the gain and thus the output power. To avoid such influences of laser frequency variations when calculating the output power versus the pump current, we slightly adjusted the beam energy for each current change to maintain a constant laser frequency. The required beam energy adjustments are smaller than $10 \%$ of the absolute beam energy. The corresponding change of the small-signal gain per round-trip is below $3 \%$. This can be shown by expanding the expression for the gain parameter $C$ given by eq. (2.47) in a Taylor series for small changes in the beam energy and substitute this in eq. (2.46). 


\subsubsection{Tuning of electron velocity}

In the previous section we gave a detailed view of the origin and the spatio-temporal dynamics of gain and oscillation in a pFEL. In the following we provide a detailed investigation of the frequency tuning of the laser upon variation of the electron velocity. As has been described in section 3.1, the electron velocity changes the dispersion of the slow-space charge wave. This should lead to a continuous variation of the crossing points with the photonic crystal dispersion and, thus, a continuous tuning of the laser output frequency. Figure 3.9 shows the expected frequency tuning from this theory vs. beam energy as a red, dashed curve.

To investigate the validity of this simple picture, we use a set of PIC calculations in which we vary the electron energy from $10 \mathrm{keV}$ to $15 \mathrm{keV}$. This corresponds to a velocity variation between $v=0.199 c$ and $v=0.243 c$. Figure 3.9 shows the calculated laser output frequencies as data points (black circles). It shows that the laser frequency does not tune continuously at all. Rather, the laser frequency tunes in a step-wise manner along a number of preferred frequencies indicated by the blue horizontal lines.

To explain the step-wise tuning in addition to frequency selection by velocity-matching, another frequency selection mechanism has to be present. We address this selection mechanism to the presence of standing waves. Similar to a standard standing-wave resonator the reflections at the planes A and B (see Fig. 3.1) impose boundary conditions such that the phase advance of the electromagnetic wave has to be a multiple of $2 \pi$ per round-trip. Hence, the laser operates only at the particular frequencies which fulfill this condition, i.e., at so-called longitudinal modes. Correspondingly, the observed tuning characteristics of the pFEL can be understood as longitudinal mode hops when the center frequency of the gain is tuned via the electron velocity. This is further supported by inspecting the results obtained with tuning in finer $(100 \mathrm{eV})$ steps around the electron energy of $12.5 \mathrm{keV}$ (center of Fig. 3.9). Below a beam energy of $11.7 \mathrm{keV}$ and above $12.8 \mathrm{keV}$ the neighboring modes - at $15.8 \mathrm{GHz}$ and $16.1 \mathrm{GHz}$, respectively - start to oscillate. Note that, at a beam energy of $12.8 \mathrm{keV}$ and $11.7 \mathrm{keV}$, the spectrum already contains a small contribution of these frequencies. However, besides mode hopping, Fig. 3.9 shows that the output frequency of a pFEL can be predicted rather well with the velocity-matching condition.

In order to explore the effects of the additional frequency selection by the resonator mirrors in more detail, we investigate in the following the steady-state output power and small-signal growth rate of the laser. We have just reported that the laser oscillation frequencies are more sharply defined by the longitudinal mode frequencies than by the velocity-matched frequencies. Thus, when tuning the electron energy the laser is for most of the electron energies forced to amplify at non-velocity-matched frequencies. We expect 


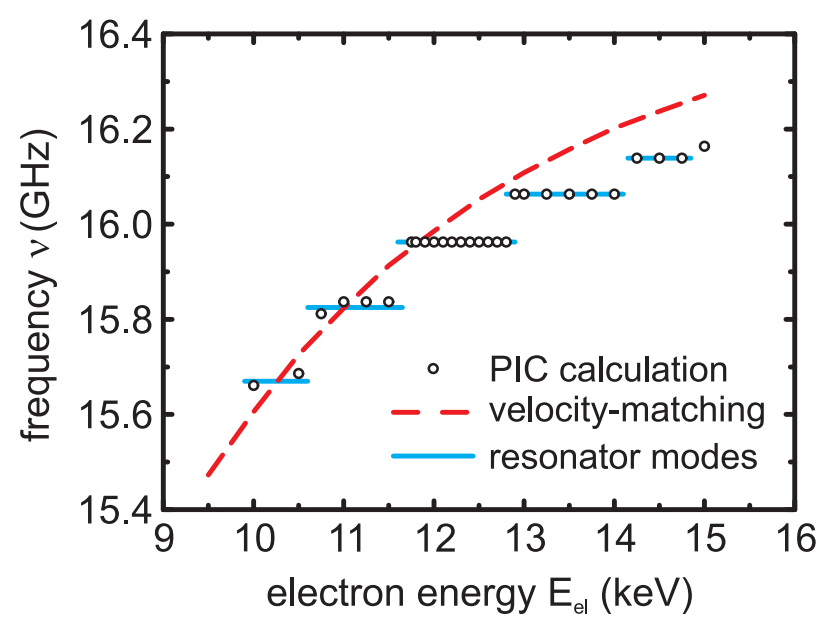

Figure 3.9: Laser frequency tuning of the pFEL by varying the electron beam energy. The frequency increases step-wise from one longitudinal resonator mode to the next. A good agreement with the frequency tuning curve deduced from velocity-matching between slow space-charge wave and photonic band structure is observed.

that this should influence laser operation and show-up as a non-optimum steady-state output or reduced small-signal gain. For an investigation of the presence and strengths of such effects, we calculate the exponential growth rate, $G_{t}^{P I C}$, and the steady-state output power, $P_{s s}$, in single longitudinal mode operation. As an example, we vary the beam energy in steps of $100 \mathrm{eV}$ around a certain center value of $12.5 \mathrm{keV}$, as discussed before in Fig. 3.9. Thereby, the laser remains oscillating in a single longitudinal mode with a frequency of $15.96 \mathrm{GHz}$. From the corresponding set of PIC calculations we determine the exponential growth rate and the steady-state power for each beam energy. The growth rate is determined with a linear regression of the logarithm of the power in a certain time window. The time window is chosen such that the goodness of the regression is better than $99 \%$, i.e., the coefficient of determination is bigger than $R \geq 0.99$. Typically, the time window then extends from $20 \mathrm{~ns}$ to $40 \mathrm{~ns}$. The steady-state power should ideally be taken when the power does not grow any longer versus time. However, fully reaching steady-state would have required many extra days of computing. On the other hand, we observe that, typically, beyond $110 \mathrm{~ns}$ the output increases by $10 \%$ at most, see for example Fig. 3.3a. As a compromise between good precision and reasonable computation time we determine, as the steady-state output, the output which is reached after a time interval of about $110 \mathrm{~ns}$.

Figure 3.10 shows the results for the growth rate and steady-state power versus beam energy, as data points. It can be seen that the maximum exponential growth rate is observed near a beam energy at about $12.3 \mathrm{keV}$, with smaller growth at both lower and 


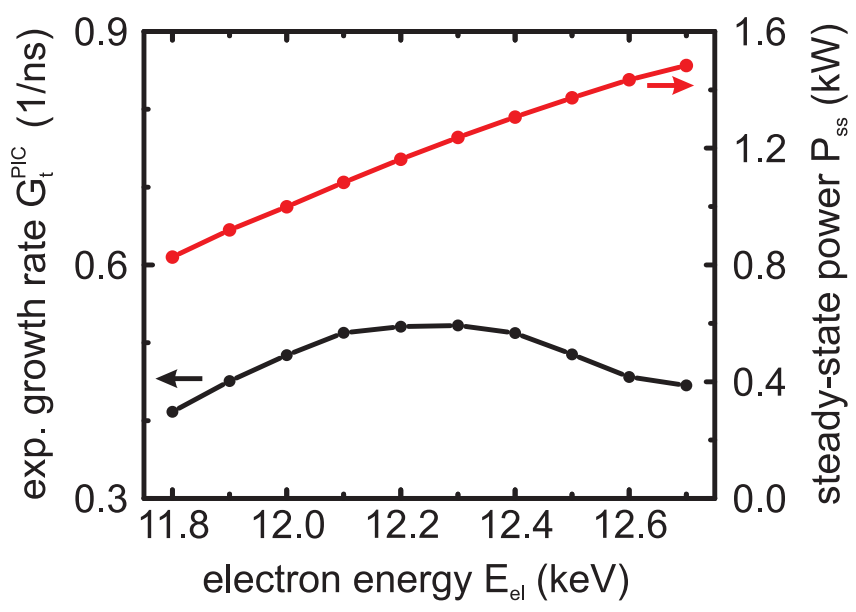

Figure 3.10: Growth rate and steady-state power versus beam energy for a longitudinal mode frequency of $15.96 \mathrm{GHz}$. (Data points are connected with lines for a better visualization.)

higher beam energies. This observation of an optimum small-signal gain can be addressed to a best velocity-matching. This interpretation is supported by a similar finding within traveling-wave tube amplifiers [89]. In these amplifiers velocity-matching is based on electrical circuits, instead of using photonic crystals to slow down the light. Furthermore, the electron beam energy is kept constant while an external input signal (seed) to be amplified is tuned in frequency. Upon tuning, the phase velocity of the seed signal varies in the slow-wave structure while the slow space-charge wave on the electron does not change. This enforces a non-velocity-matched amplification of the seed. For travelingwave tubes it is reported that the highest small-signal gain is found close to ideal velocity matching, while the small-signal gain decreases for mismatched velocities [89]. Indeed, this is very similar to the properties we have just reported for the pFEL.

In contrast to the exponential growth rate, the steady-state output power shows a different characteristic upon beam energy tuning. As can be seen from Fig. 3.10, the maximum steady-state power is detected at the highest possible electron energy in the inspected interval of single mode oscillation. We address this finding to the fact that electrons with a higher energy can loose a bigger fraction of their energy before the electromagnetic field is capable to overtake the electrons. This argument is essentially the same as used to explain the origin of gain saturation discussed above in Fig. 3.7. Following our argument, the extra amount of emitted power at the upper edge of the interval should be less or equal to approximately $10 \%$ of the extra pump power offered at the upper edge via a higher energy. However, we observe a much higher increase in the output power by about $55 \%$ at the upper edge. Thus, we expect that more complicated effects, such as 
based on effective refractive index contribution of the driving electron beam might cause this increase in output power [78].

\subsection{Comparison of particle-in-cell calculations to lin- earized theory}

Investigating a pFEL using PIC methods can reveal important basic information about, e.g., the small-signal gain, the threshold, and the frequency tuning of the output. However, on the other hand, a PIC modeling also suffers from strong limitations of feasibility due to extensive computational demands and thus long calculation times. For instance, to calculate the few data points presented in the previous section a total computation time of about three weeks was necessary, although we have only studied a single, specific design of a pFEL. In comparison, linearized models offer a much higher amount of results within a much shorter time. Within a few hours it is possible to give results on small-signal gain, threshold current and frequency tuning for a wide range of beam parameters. This advantage is essential when the suitability of different kinds of photonic crystals has to be compared, and a wide range of pump parameters has to be explored, such as for preparing an experimental demonstration. However, as linearized methods are based on severe approximations, a validation is required to make sure that these methods can be safely applied for design purposes. A study concerning this validation has never been presented for pFELs.

In this section we compare the results of PIC calculations with small-signal gain and threshold calculations based on the linearized theory described in section 2.6. Recall that the linearized theory is essentially assuming that the photonic crystal can be replaced by an effective dielectric medium. The corresponding effective refractive index is such that the wave number inside the medium $k_{P h C}$ corresponds to a phase velocity which is velocity-matched. The complex electromagnetic field distribution of a photonic crystal comprises a large set of different wave numbers, but is simply replaced by a single wave number. This wave number $k_{P h C}$ represents only a part of the complete field distribution. The small-signal gain per wavelength is then estimated as spatial overlap integral of this part of the field distribution with the electron beam through eqs. (2.46) and (2.47).

To apply these equations we need to determine the velocity-matched spatial harmonic amplitude $E_{z, m}\left(x, y, k_{0}\right)$ and the velocity-matched wave number $k_{z, P h C}$ for the given electron beam parameters $U_{0}, I_{0}$ and $S$ (cross sectional area of the beam). We have shown in section 3.2 how to calculate the velocity-matched wave number using an eigenmode solver. This eigenmode solver also computes the total spatial field distribution of the correspond- 
ing photonic crystal mode throughout the unit cell. From this total field distribution the velocity-matched part, i.e., the velocity-matched spatial harmonic amplitude, can be calculated by a spatial Fourier transform along $z$. For more details about the implementation of this method the reader is referred to [166].

To compare the gain per wavelength $G_{\lambda}^{\text {lin }}$ retrieved from the linearized theory to the exponential growth rate obtained from PIC calculations $G_{t}^{\mathrm{PIC}}$, we need a method to compare both quantities. ${ }^{1}$ Thus, we aim to transform a gain in time $t$ into a gain per distance $z$. We do this by showing how the exponential growth rate $G_{t}^{\text {lin }}$ compares to the gain per wavelength $G_{\lambda}^{\text {lin }}$.

We assume that, one can interpret a pFEL oscillator based on a resonator as cascade of pFEL amplifier sections. Each amplifier section represents a certain round-trip in the laser resonator. We assume that, at the beginning of the first round-tip, some small initial power, $P_{\text {init }}$, is present inside the first amplifier section. As the amplification process is uni-directional, the power at the end of the $n^{\text {th }}$ section is:

$$
P_{\text {intra }}\left(n L_{r e s}\right)=\left(\frac{1}{9} R\right)^{n} P_{\text {init }} \exp (2 \pi \sqrt{3} C N)^{n} \quad \text { with } \quad N=\frac{L_{r e s}}{\lambda_{P h C}}
$$

Here, $N$ is the number of velocity-matched wavelengths that fit into the length of the gain section, $L_{r e s}$, and $R$ are the reflection of the out-coupling mirror. The factor $\frac{1}{9}$ expresses that a free electron based amplifier and, thus, here each amplifier section, effectively amplifies only a ninth of the injected power, as was discussed in section 2.6. The time the power of the electromagnetic wave needs to complete $n$ round-trips depends on the group velocity, $v_{g}$, as:

$$
t_{n}=2 n \frac{N \lambda_{P h C}}{v_{g}}
$$

From the PIC calculations we calculate the logarithmic power versus time $t$ at the output port (see Fig. 3.1) to quantify the exponential growth, $G_{t}^{\mathrm{PIC}}$, in the small-signal regime. We can also use eqs. (3.4) and (3.5) to calculate the logarithm of the power at the output port as a function of time. After some algebraic steps we find:

$$
\ln \left[P_{C}\left(t_{n}\right)\right]=\ln \left(P_{\text {init }}(1-R)\right)+\frac{v_{g}}{2 \lambda_{P h C}}\left[2 \pi \sqrt{3} C+\frac{\ln (R)-\ln (9)}{N}\right] t_{n} .
$$

Here $C$ is the gain parameter and depends on the photonic crystal and the electron beam, as given by eq. (2.47). In the case we investigate here the out-coupling is weak, thus

\footnotetext{
${ }^{1}$ For better clarity we will in the following denote results from the linear theory with a superscript lin and from the PIC calculations with a superscript PIC.
} 
$R \approx 1$ and the logarithm of $R$ is close to zero. With this simplification we conclude that the growth rate $G_{t}^{\text {lin }}$ is connected to the gain parameter $C$ via

$$
G_{t}^{\operatorname{lin}}=\frac{v_{g}}{2 \lambda_{P h C}}\left[2 \pi \sqrt{3} C-\frac{\ln (9)}{N}\right]
$$

From this, we determine the relationship between gain per wavelength and exponential growth rate in the linearized model as:

$$
G_{\lambda}^{\operatorname{lin}}[d B]=47.3 C=\left[\frac{2 \lambda_{P h C}}{v_{g}} G_{t}^{\operatorname{lin}}+\frac{\ln (9)}{N}\right] 10 \log _{10}(e)
$$

where, $e=2.718$ is Euler's number.

Figure 3.11 shows the gain per wavelength $G_{\lambda}$ versus the electron velocity calculated. The red curve is calculated from the linear theory, via eqs. (2.46) to (2.49). The black data points show the small-signal gain per wavelength $G_{\lambda}^{\mathrm{PIC}}$ deduced from the PIC calculations, when inserting the growth rate $G_{t}^{\mathrm{PIC}}$ in eq. (3.8). To exclude possible resonator effects, the figure shows only the maximum value of the small-signal gain found for each longitudinal resonator mode.

We observe that the small-signal gain increases towards higher beam energies in both models. Also the absolute values show a satisfying agreement, although the linear theory slightly overestimates the gain by 0.2 to $1.0 \mathrm{~dB}$. We address this deviation to a number of effects that can reduce the gain, but are neglected in the linearized model. Considering that the simple model neglects energy spread of the electron beam, Coulomb repulsion, the influence of other spatial harmonics and effects that are related to a small group velocity $[89,136,160,170,171]$ the found agreement is actually rather good.

Another way to compare the two models, now including the loss per round-trip explicitly, is to determine the threshold pump current predicted by the linearized model. The threshold is reached when the gain per round-trip exceeds the losses per round-trip, such that the laser can start to oscillate. The loss per round-trip is determined by the out-coupling loss of about $5 \%$ per round-trip ( $0.22 \mathrm{~dB}$ per round-trip) and the bunching loss of $9.54 \mathrm{~dB}$. The gain per wavelength is computed using the eqs. (2.46) to (2.49). The result is a value of about $2.25 \mathrm{~dB}$ per wavelength using a beam energy of $12.5 \mathrm{keV}$ and a beam current of $1 \mathrm{~A}$. As the velocity-matched wavelength is $\lambda_{P h C}=3.78 \mathrm{~mm}$, about 13 wavelengths fit into the resonator with a length of $L_{\text {res }}=50 \mathrm{~mm}$. Consequently, the net amplification per round-trip amounts to about $29.25 \mathrm{~dB}$, which is well above the total loss per round-trip of about $9.76 \mathrm{~dB}$. To equalize gain and loss this yields a threshold current of about $I_{t h}=37 \mathrm{~mA}$, while the extrapolation based on PIC calculations predicts 


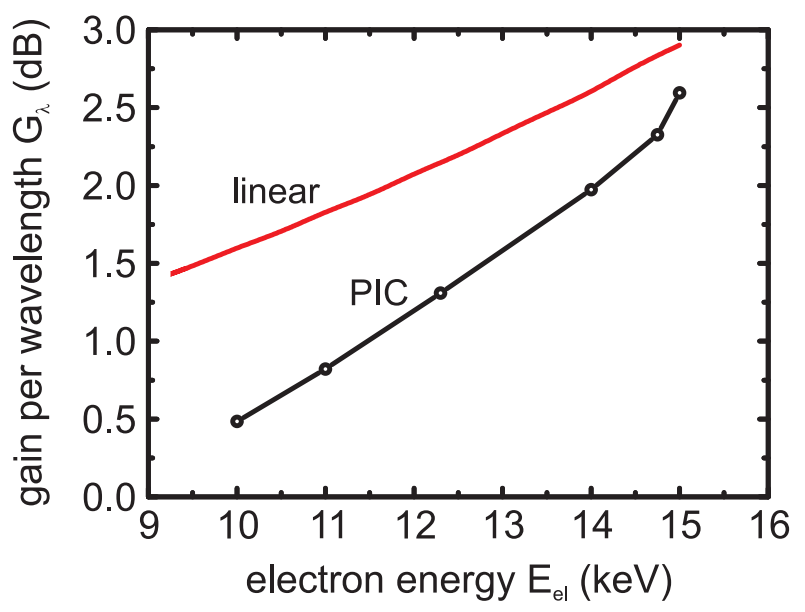

Figure 3.11: Comparison between small-signal gain computed by a linear theory and deduced from the PIC calculation shown in section 3.3. (Data points are connected with lines for a better visualization.)

a threshold current of about $I_{t h}=140 \mathrm{~mA}$ (section 3.8). As expected the linear theory slightly underestimates the threshold as it overestimates the small-gain per wavelength.

In conclusion, although the linear theory neglects: the influence of the energy spread of the electron beam, Coulomb repulsion, the influence of other spatial harmonics, and effects that are related to a small group velocity $[89,136,160,170,171]$ we have shown that a strongly simplified linearized theory, as presented in section 2.6, reasonably estimates the threshold current and small-signal gain of a pFEL. Therefore, the linearized theory appears an appropriate and very effective method for comparing pFELs based on various different photonic crystal geometries.

\subsection{Conclusion and outlook}

In conclusion, we have presented the first comprehensive numerical analysis of the linear and nonlinear properties of pFELs, based on a resonator. Although the detailed modeling based on PIC calculations is rather tedious, it has allowed us to physically understand and analyze the dynamics of electromagnetic field amplification and oscillation with the associated dynamics of the electron beam within a simple working principle. This working principle is based on the constructive interference of wavelets emitted from the photonic crystal as a response to the electron propagation, and the decelerating and accelerating feedback of the emitted waves on the electron motion. Choosing a specific example of a $\mathrm{pFEL}$ in the microwave range we investigated some of the basic laser properties in more detail. These are the dynamical growth of the electromagnetic field, the spatio-temporal 
electron beam dynamics, the laser threshold, the output power scaling versus the pump current and the tuning of the operation frequency.

We find that, after the onset of the electron beam, the initial passing electrons generate spontaneous emission which leads to a weak output with fluctuating amplitude and phase. As the photonic crystal slab we study has a particular mode with a phase velocity component matched to the electron velocity and with a longitudinal electric field component, the fluctuating power induces small electron bunching. The electron bunching subsequently enhances the emission into that photonic crystal mode, with the same phase. This is equivalent to stimulated emission. If the electron beam is sufficiently strong, the gain provided by this mechanism exceeds the losses and exponential growth over several orders of magnitudes sets in. Eventually, the gain saturates, as many electrons lose too much kinetic energy to remain velocity-matched. This means that many electrons start absorbing energy instead of amplifying it. When steady-state operation is reached, the output spectrum typically comprises of a single frequency. In our example, this frequency lies around $16 \mathrm{GHz}$, with a resolution limited bandwidth of about $2 \mathrm{MHz}$. The output power of the laser in steady-state is about $1.5 \mathrm{~kW}$, scales linearly with the applied pump current, and the threshold current is about $I_{t h}=140 \mathrm{~mA}$. We further verify that, by changing the electron velocity, the laser frequency is tunable following the velocity-matching of the slow space-charge wave and the frequency dependent electromagnetic phase velocity of the excited photonic crystal mode.

To significantly reduce the number of time consuming numerical PIC calculations for assessing the suitability of photonic crystals for use in a pFEL we finally presented the comparison with a simple, linearized slow-wave model. We have verified the approximate validity of this simplified model by observing that most important laser properties can be predicted with a satisfying agreement. For the chosen example of a pFEL, the small-signal gain per wavelength is found to be as close as $0.2 \mathrm{~dB}$ to the result of comprehensive PIC calculations. Also the predicted laser threshold of $I_{t h}=37 \mathrm{~mA}$ is reasonably close to the laser threshold found in PIC calculations $\left(I_{t h}=140 \mathrm{~mA}\right)$. 


\section{4 \\ Photonic free-electron laser pumped by multiple electron beams}

\subsection{Introduction}

Electromagnetic waves with high power $(P>1 \mathrm{~W})$ find numerous applications at various frequency ranges. For example, remote sensing [172-175], material processing [13, 14, 176182] and cancer treatment [183-186] use lasers from around the microwave up to the visible range and beyond. Typical laser applications demand supplying the power with a high spatial brightness, i.e., the total power is delivered from a small mode area in the focus into a small solid angle in the far-field. In addition, the laser should preferably provide a high spectral brightness by restricting the oscillation to a single frequency. The highest spectral brightness is achieved when only a single longitudinal mode oscillates. Thus, for a laser resonator based on free-space propagation the highest brightness at a given power is achieved when the output is concentrated in a single longitudinal mode with a $\mathrm{TEM}_{00}$ transverse mode profile [169]. The equivalent statement for a waveguide laser is that the output power is delivered in a single transverse mode and a single longitudinal mode, as such output can be fully converted into a single frequency $\mathrm{TEM}_{00}$ mode in free-space using suitable diffractive optical elements. In the previous chapter we predicted that a 
pFEL in the microwave regime, even if based on a single electron beam, already emits a substantial power of $P_{s s}=1.5 \mathrm{~kW}$ in a single transverse and longitudinal waveguide mode. With such power levels, pFELs seem to be promising for a variety of high power applications at microwave frequencies.

Nevertheless, an even higher promise lies in the fundamental frequency scalability of pFELs which is based on the scale invariance of Maxwell's equations - see chapter 2. Reducing the spatial period of the crystal by a chosen factor would increase the laser frequency with the same factor. However, the output power only remains constant if the other laser parameters are kept constant as well. The latter means particularly the pump power, which is given by the product of the voltage (proportional to the beam energy) and the current of the electron beam that pumps the laser. However, the required scalability of electron beams is fundamentally limited as is discussed in the following.

When downscaling the characteristic length scale of the photonic crystal, by a certain, desired factor, the cross sectional area of the vacuum channels in the crystal through which the electrons stream becomes inevitably smaller. Thus, when attempting to maintain a constant pump current for a constant output power the current density would have to increase quadratically with the scaling factor. Thereby, also the mutual Coulomb repulsion of the electrons would strongly increase. Already at low scaling factors it is complicated to compensate space-charge forces with a magnetic guiding field. Upon scaling down the photonic crystal features, the required magnetic field strength would go beyond any feasible values [65]. A second problem is, that the emission density of cathodes is fundamentally limited. Typically, for a long lifetime the emission density should not exceed $10 \mathrm{~A} / \mathrm{cm}^{2}[63,187,188]$. Third, space-charge effects inside high current density beams counteract bunching which eventually limits the gain or leads to chaotic oscillations of the output power $[89,189]$. In conclusion, this means that the current density cannot be scaled and upon frequency scaling the available pump power and also the output power of the laser will reduce. At some point the pump current would be too low to reach the threshold pump current of the laser and no oscillation will set in anymore.

One might argue that the pump power deployed through a vacuum channel of downscaled size can be maintained also with increasing the beam energy. When the beam current drops quadratically with the scaling factor, one could maintain the power by quadratically increasing the beam energy. Indeed, high power microwave tubes, such as the Klystron or Gyrotron can generate megawatt power levels in a single mode by using high beam energies in addition to high currents [190, 191]. However, such scaling via the beam energy immediately leads to extremely high (relativistic) energies, the generation of which requires large-scale electron sources. Thus, to enable a frequency scaling of pFELs 
a new approach needs to be devised which enables to deploy the same pump power into a photonic crystal with smaller channels. More specifically, in order to not loose brightness but gain brightness as discussed above, this approach needs to be such that the laser output is still concentrated in a single transverse mode.

Our solution to maintain the pump power upon down-scaling the crystal is to spatially distribute the pump current in the transverse direction. Effectively, when the size of the vacuum channels in the photonic crystal becomes smaller for an up-scaling of the frequency, the decreased pump current per channel is compensated by pumping through an increased number of channels. On a more fundamental level, i.e., before even attempting to upscale the frequency, this is equivalent to the following statement. The output power from a pFEL at a fixed frequency should increase when the pump current is increased via a higher number of pump beams in a manner that does not lead to multiple transverse mode oscillations. The latter condition is of central importance because only if the output power of the pFEL remains concentrated in a single mode will the brightness increase with the pump power. Note that increasing the number of pump beams means that the pumped volume also increases. Corresponding studies of power up-scaling via increasing the pumped volume have already been performed earlier with relativistic backward-wave oscillators using corrugated waveguides with a large diameter [95, 101, 192]. However, the increased pumped volume leads simultaneously to oscillation in multiple transverse waveguide modes [95, 101, 192]. This result is actually typical for most types of lasers. For example, when a larger volume is pumped in solid-state lasers or diode lasers, it is typically found that the output is distributed over an increased number of transverse modes [193-196]. Thus, while the power can be increased via pumping a large volume, the high brightness associated with single spatial mode oscillation is typically lost.

In this chapter we demonstrate that, in contrast to the named other lasers, the oscillation of pFELs can remain restricted to a single mode, although the power increase is achieved via pumping a larger volume. This difference in scaling is imposed by the periodic structure of photonic crystals which enforces a single spatial and spectral mode output. In a pFEL, a larger volume becomes available when increasing the number of transverse crystal periods. In section 4.2 we investigate a specific example of such a pFEL and consider pumping with an increasing number of (up to seven) electron beams. PIC calculations in section 4.3 show that for this pFEL a power up-scaling in a single longitudinal and transverse mode is achieved, which is equivalent to up-scaling the brightness. In section 4.4 we illustrate that the emergence of single-mode oscillation arises from the combination of spatial mode selectivity in photonic crystals with mono-energetic electron beams. The latter provides gain competition that leads to a condensation into a single 
mode, as found in lasers with homogeneously broadened gain. To generalize the specific results obtained from the pFEL investigated, we discuss possible extrapolations of the numerical results towards many beams. We conclude the chapter with an outlook how the power scaling might be used to generate powerful $\mathrm{THz}$ radiation using pFELs.

\section{$4.2 \quad$ pFEL model and dispersion}

The pFEL model we investigate here is shown in Fig. 4.1. The laser design is a straightforward extension of the single-beam pFEL presented in chapter 3. We increase the number of photonic crystal periods in the transverse $x$-direction, from a value of two to eight, which creates a seven-times larger pump volume. The other parameters describing the laser geometry remain unchanged compared to the single-beam pumped pFEL shown in chapter 3. For instance, again 20 unit cells of the photonic crystal slab are enclosed in the propagation direction $z$ by a resonator. This resonator is formed by a metallic mirror at plane A with $R=100 \%$ reflectivity and the interface between the photonic crystal slab and the empty rectangular waveguide, labeled B in Fig. 4.1. The reflectivity of the interface is determined to be about $R>85 \%$, where its precise value depends on the photonic crystal mode considered (see appendix A). As indicated in the figure, the photonic crystal slab allows the laser to be pumped by up to seven electron beams labeled as 1 through $4 \mathrm{a}$ and 4b. By successively turning on the current in an increasing number of pump beams, the total pump power can be increased while the current in each beam, and thereby also the current density, is held constant. This method avoids the complexities of increasing the pump power via an increased current density of a single electron beam, as discussed in section 4.1. The electron beams are injected at plane A with an energy of $E_{e l}=13 \mathrm{keV}$ each. The beam diameter is chosen as $2 \mathrm{~mm}$ and the current as $I=1 \mathrm{~A}$ for each beam. At plane $\mathrm{C}$, the output port, the electromagnetic field is decomposed into the empty waveguide modes. In order to relate the empty waveguide modes to the photonic crystal modes, we investigate in appendix A the coupling between the two different sets of modes at the interface B. We show that each photonic crystal mode couples only to a single empty waveguide mode, and vice versa. Therefore, each mode inside the crystal can be unambiguously associated with a certain waveguide mode, and we do not need to distinguish between both sets of modes in this chapter.

Due to the large volume of the slab, it can be expected that many transverse photonic crystal modes exist in the devised operating frequency range around $16 \mathrm{GHz}$. To assess the number of modes that might start oscillating for the multi-beam pFEL we determine how many transverse modes are velocity-matched for the considered frequency in the lowest 


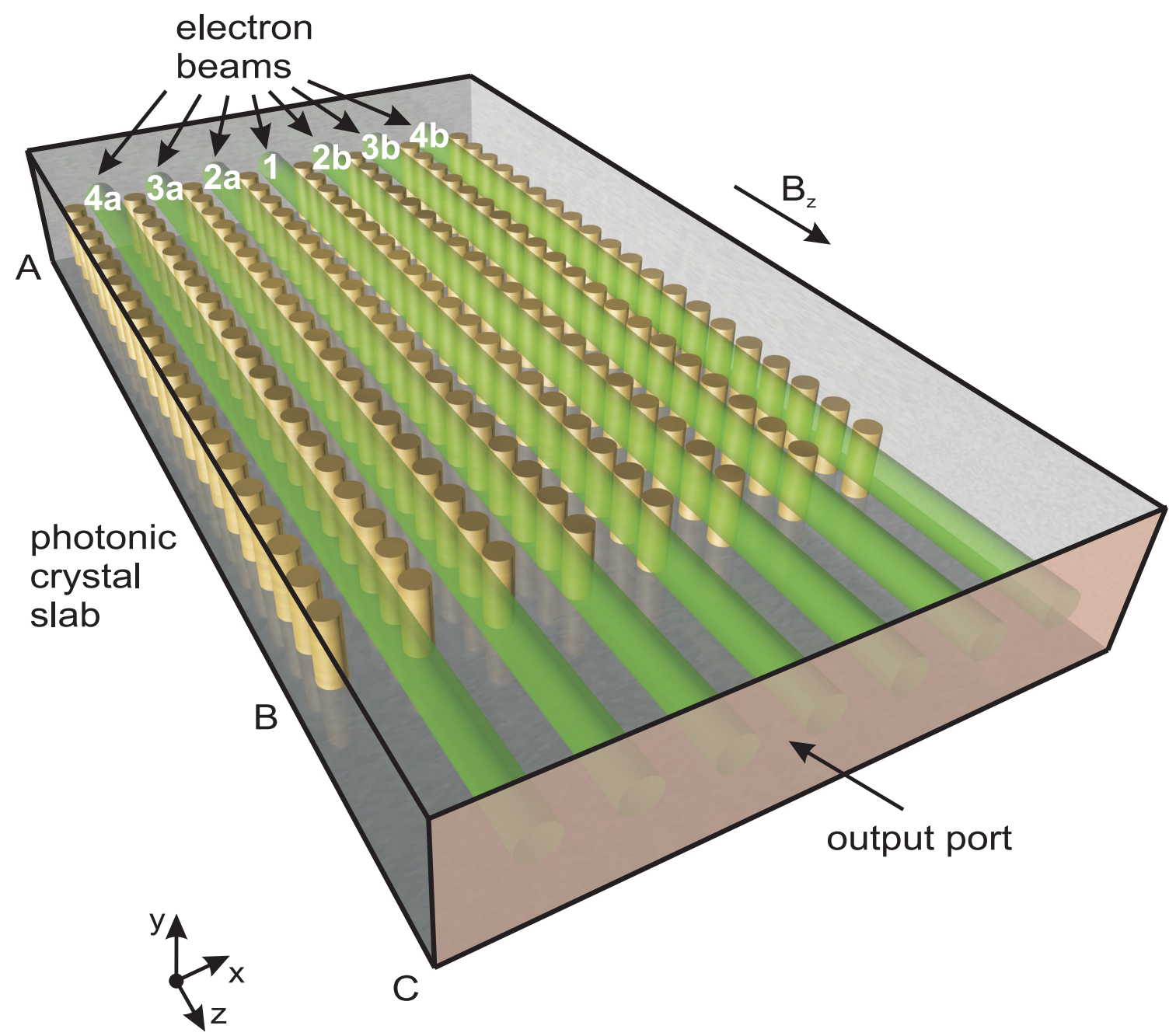

Figure 4.1: Schematic overview of a multi-beam pFEL. Up to seven electron beams enter at $\mathrm{A}$ into a photonic crystal slab that consists of 20 unit cells (see Fig. 4.2a) to provide gain. The gain section is placed inside a resonator, formed by a metallic mirror at A and the transition between a rectangular waveguide and the photonic crystal slab at $\mathrm{B}$. At plane $\mathrm{C}$, the so-called output port, the generated electromagnetic fields are sampled in time. Throughout the model the electron beam is guided by a static homogeneous magnetic field of $B_{z}=0.5 \mathrm{~T}$. 
(a)

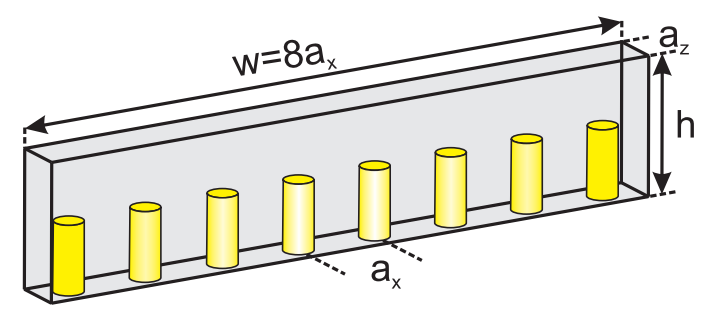

(b)

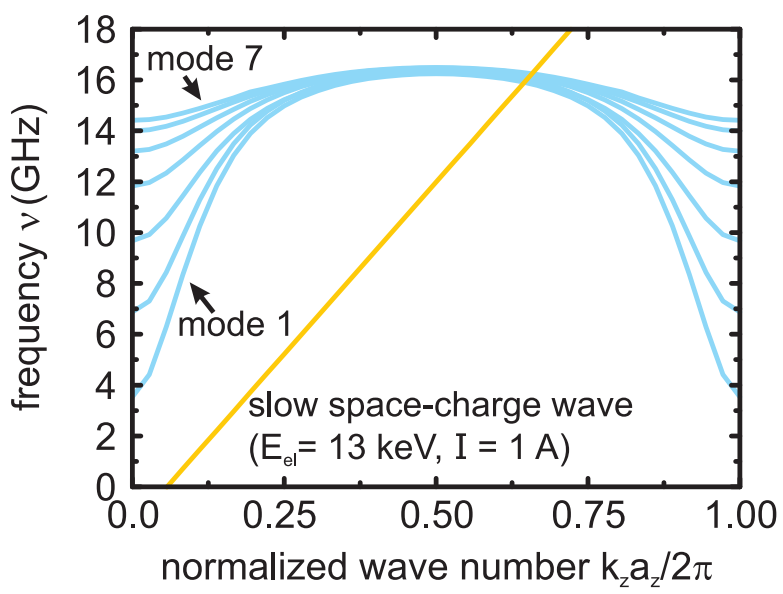

Figure 4.2: (a) Unit cell of the metallic photonic crystal slab, $a_{x}=4.2 \mathrm{~mm}, a_{z}=2.5 \mathrm{~mm}$, $h=8 \mathrm{~mm}$, rod height $h_{p}=4 \mathrm{~mm}$, rod radius $r_{p}=0.75 \mathrm{~mm}$, (b) Band diagram of the photonic crystal slab and dispersion of the slow space-charge wave for the electron beams to find at which frequencies velocity matching occurs.

possible spatial harmoinc. This number of velocity-matched modes is found by comparing the dispersion of the slow-space charge wave with the photonic crystal modes. The phase velocities of photonic crystal modes and the slow space-charge waves are matched when their dispersions curves intersect. We calculate the slow space-charge dispersion via eq. (2.24). The photonic crystal dispersion is found by computing the eigenmodes of the unit cell of the photonic crystal slab (Fig. 4.2a). For this we use again an iterative eigenmode solver [141] and approximate, as previously, all metallic parts as perfect conductors. Figure $4.2 \mathrm{~b}$ shows the calculated dispersion for the lowest-frequency modes of the photonic crystal slab, as well as the dispersion of the slow space-charge wave. In contrast to the single-beam pFEL, we observe that now up to seven transverse photonic crystal modes exist around $16 \mathrm{GHz}$, which is due to the large width of the photonic crystal slab. We label these modes with increasing cut-off frequency, i.e., the lowest-frequency mode is termed mode 1 , the next mode 2 and so on. All of the seven modes around $16 \mathrm{GHz}$ are velocity-matched to the slow-space charge wave in the first spatial harmonic. This enables that all these different modes have the potential to be amplified, which may result in a spectrally and spatially multi-mode output with a correspondingly low brightness. Whether or not such multi-mode emission occurs depends on the competition of photonic crystal modes for the available gain from the electron beams. This gain competition might either result in an output that contains a single mode or multiple modes. To comprehensively investigate the effect of gain competition, a fully nonlinear description of the pFEL dynamics is required. 


\subsection{PIC calculations}

PIC calculations allow to investigate the growth during start-up including the gain competition between modes. Hence, PIC calculations are ideal to study whether both power up-scaling and single-mode operation can be achieved simultaneously. To investigate power scaling we have performed four sets of PIC calculations with a step-wise increase in pump power via adding more electron pump beams. First, the laser is pumped with a single electron beam in the center - labeled 1 in Fig. 4.1. Then we model the same laser with three adjacent beams turned on $-1,2 \mathrm{a}$ and $2 \mathrm{~b}-$, five adjacent beams - 1, 2a, 2b, 3a and $3 \mathrm{~b}-$, and finally with the maximum possible amount of seven beams. For inspecting the dynamical growth of the electromagnetic field, we chose as initial conditions for the PIC calculation that no radiation field and particles are present inside the resonator. After $t=0 \mathrm{~ns}$, the current in each of the electron beams is ramped up to a constant final value of $1 \mathrm{~A}$ by increasing the number of injected super-particles from zero towards 121 per beam, during the first 900 time steps, i.e., within $0.3 \mathrm{~ns}$.

Figures $4.3 \mathrm{a}-\mathrm{d}$ show in semi-log plots the power growth at the output port vs. time with different numbers of pump beams turned on, i.e., with single-beam pumping (a) to seven-beam pumping (d). As the beam energy and current per beam is kept constant, the pump power increases linearly with the number of beams. The semi-log plots show the three transverse modes with the highest output power for each pump scheme. Common for all pump powers are the following three findings. First, the output power in all modes is initially weak and noisy. Second, after a certain time exponential growth occurs for all modes. Note that, due to the limited dynamical range that can be displayed for an overall comparison, the power level of spontaneous emission and the onset of exponential growth is not visible for the weakest modes. The most important finding from all figures is that, while initially all modes grow, eventually the steady-state output is clearly dominated for all pump powers by a single transverse photonic crystal mode, which is always mode 1. For all pump powers investigated, the output power in mode 1 is at least almost two orders of magnitude higher than in the other modes. On a linear scale, at least $95 \%$ of the total output power is contained in mode 1 . When looking at the total steady-state output power we can show that it grows close to linearly with the applied pump power. However, we postpone a more detailed study of the power scaling to section 4.5. For now, to investigate whether the increased power also corresponds to an increased brightness, we need to prove that the power contained in the single transverse mode, mode 1 , is also completely contained in a single longitudinal mode of the laser resonator.

Potential oscillation in multiple longitudinal modes should show up as a laser output that contains multiple frequencies. A suitable way to identify the number of oscillating 
(a) one beam

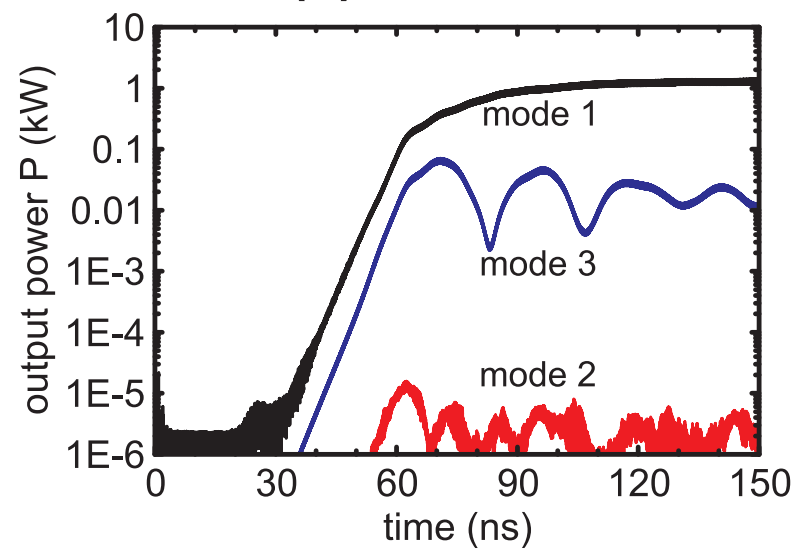

(c) five beams

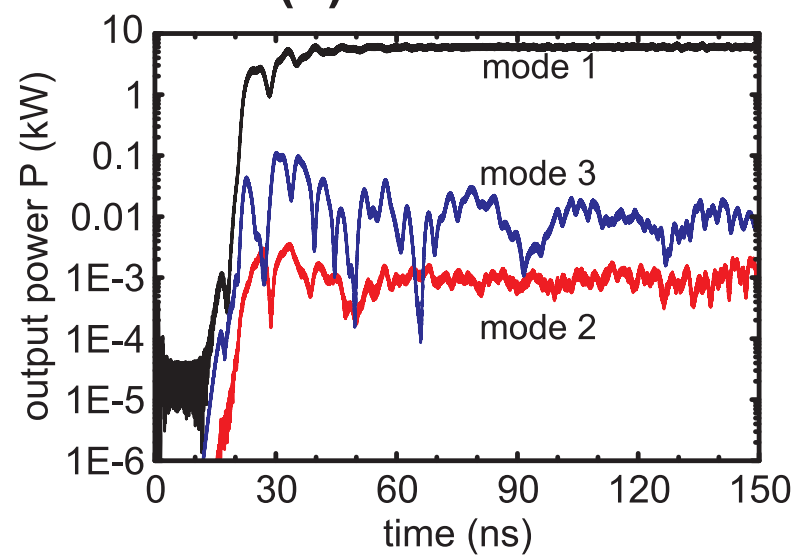

(b) three beams

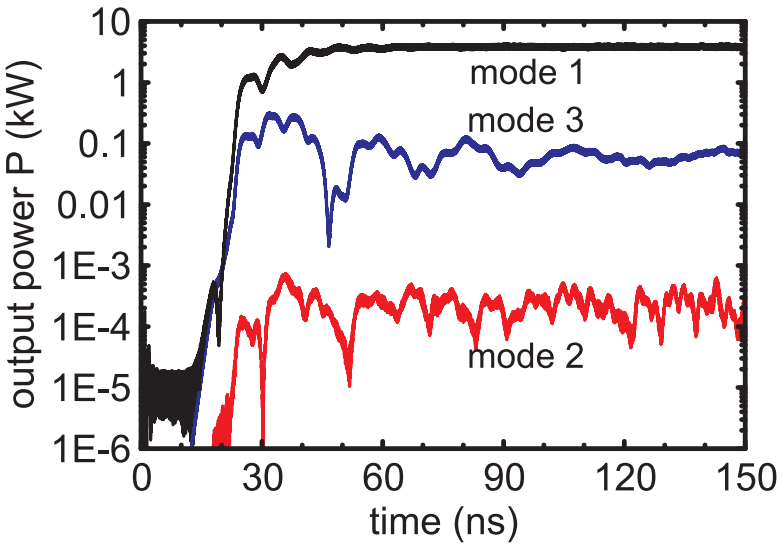

(d) seven beams

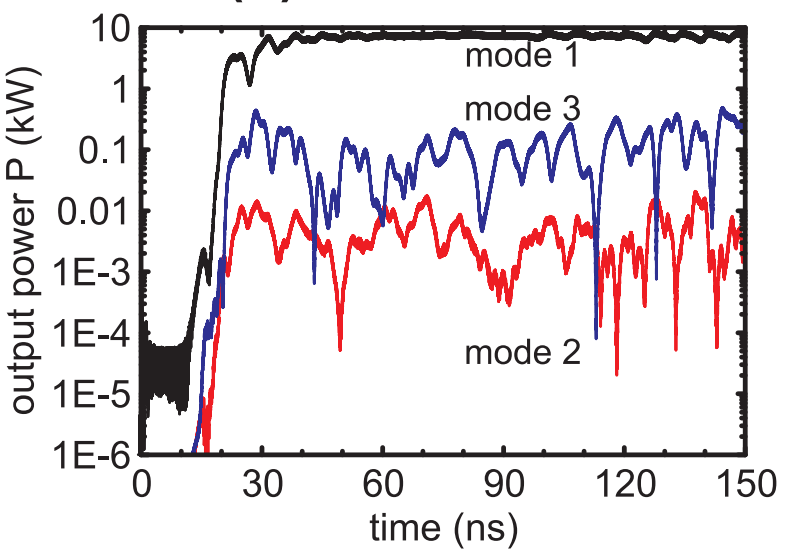

Figure 4.3: Temporal power growth of the three strongest modes at the output port for a multi-beam pFEL. The different sub-figures a-d show the output power when pumped with (a) one beam, (b) three beams, (c) five beams and (d) seven beams.

longitudinal modes in the dominant transverse mode, mode 1 , is thus to inspect the power spectrum of this mode. To calculate this spectrum we take the square of the magnitude of the Fourier-transform of the electromagnetic field sampled for mode 1 at the output port. Figure $4.4 \mathrm{a}-\mathrm{d}$ shows the power spectra for the different pump schemes. It is observed that the spectrum of mode 1 consists basically of a single sharp maximum, i.e., the dominating mode 1 oscillates at a single frequency. This frequency is around $15.87 \mathrm{GHz}$ and the spectral bandwidth (FWHM) is less than $10 \mathrm{MHz}$. Note that the spectral distance between different longitudinal modes of the resonator is more than $0.1 \mathrm{GHz}$. This spectral distance makes it easily detectable if more than a single longitudinal mode of the resonator would be excited, as the spectral resolution of the Fourier-transform is much smaller (about $7.5 \mathrm{MHz}$, given by the finite time window of the data in Fig. 4.3). From the 
(a) one beam

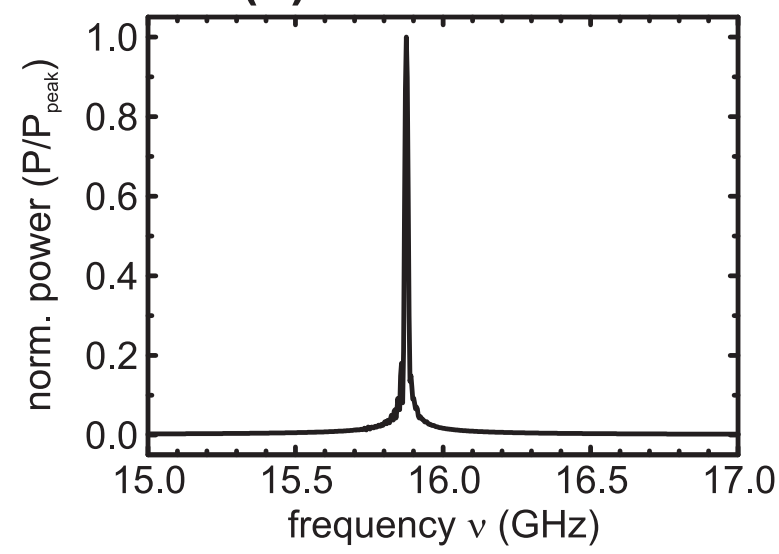

(c) five beams

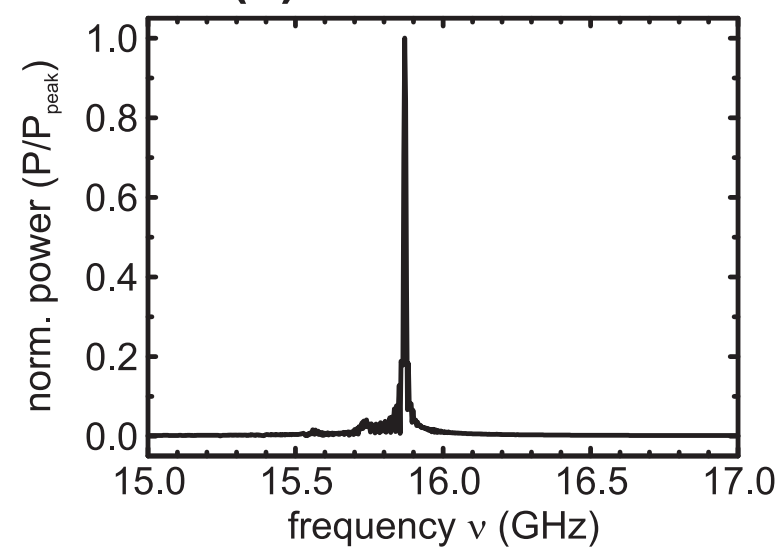

(b) three beams

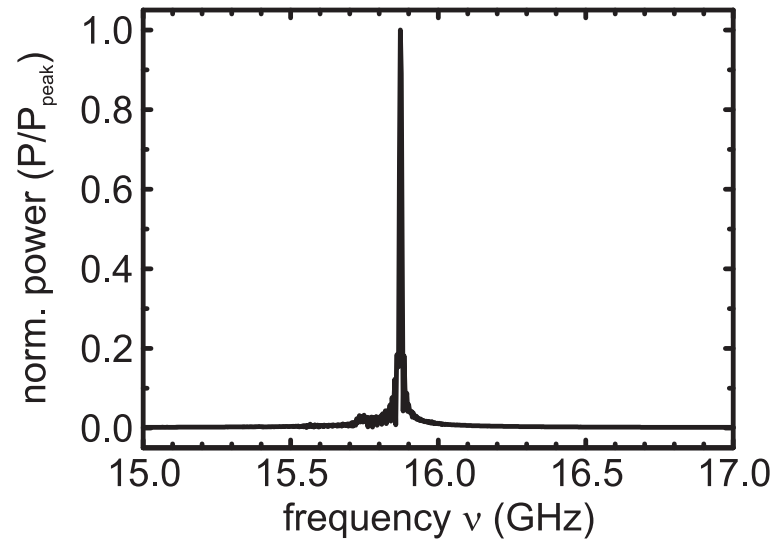

(d) seven beams

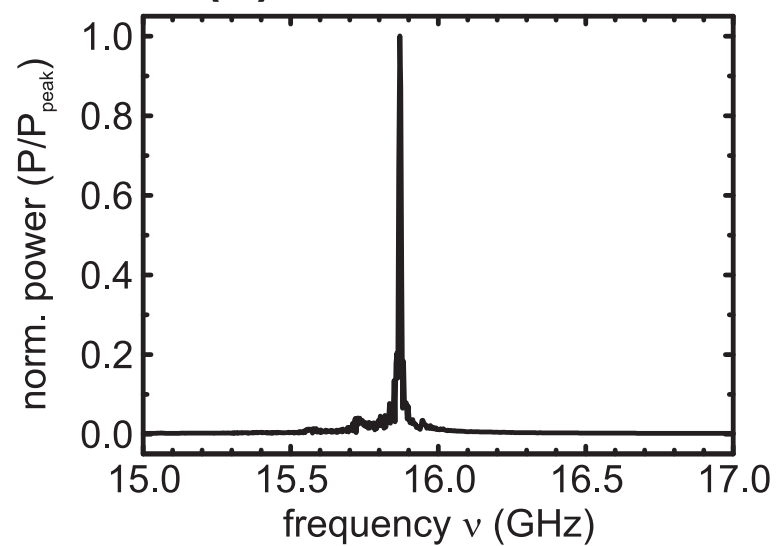

Figure 4.4: Power spectrum of the strongest transverse mode, mode 1, at the output port when driven by an increasing number of electron beams. The power spectrum is normalized to the maximum power of mode 1 for each pumping scheme.

data in Figs. 4.3 and 4.4, we can conclude that the laser is clearly operating in a single transverse mode and also in a single longitudinal mode, except for deviations of at most a few percent of power. This means that the demonstrated power up-scaling via pumping with multiple electron beams has also increased the brightness of the output.

\subsection{Mode competition}

The results of the PIC calculations presented in the previous section predict that the output of the investigated multi-beam pumped pFEL eventually is dominated by a single transverse and longitudinal mode and also grows with the number of pump beams. A wellknown mechanism that leads to steady-state single-mode oscillation in potentially multi- 
mode lasers is gain competition. While the observed power up-scaling of the brightness is very desirable for applications, a fully systematic analysis of gain competition is hindered by limited computational resources for performing the required PIC calculations and its "Blackbox" character. These circumstances, presently, limit the following discussion of gain competition in multi-beam pFELs. However, some important conclusions regarding the underlying physical mechanisms can still be drawn.

To find the underlying physical mechanism for explaining the observed mode selection, we recall that a mono-energetic electron beam amplifies electromagnetic fields in a similar fashion as, e.g., a solid-state laser medium with a homogeneously broadened gain [197, 198]. Homogeneous broadening of the gain in solid-state lasers means that each electron in the upper laser level is capable to amplify all frequencies in the gain bandwidth [169]. This implies that the growth of a certain mode diminishes the gain for any other competing mode. In this case, only the mode with the highest net small-signal gain survives the mode competition in steady-state, even when the gain advantage is extremely tiny [199]. This is sometimes termed as "spectral condensation", "mode condensation" or simply described by the phrase: "The winner takes it all."

To test this hypothesis for our case we derive the net small-signal gain per round-trip for each mode for a comparison. As the reflection loss is similar for all of the considered modes (see appendix A) and also the wavelength of the considered modes is almost equal, we can simplify the comparison to the small-signal gain per wavelength $G_{\lambda}$. For pumping with a number of $t$ electron beams, each with a cross-section $S_{b}$ and a current per beam $I_{b, 0}$, the small-signal gain per wavelength becomes [200]:

$$
G_{\lambda}[d B]=47.3 C[\mathrm{~dB}]=47.3\left(\frac{\sum_{b=1}^{t} \int_{S_{b}}\left|E_{z, m}\left(x, y, k_{0}\right)\right|^{2} \mathrm{~d} \mathbf{S}_{b}}{2 k_{z, P h C}^{2} W v_{g} t S_{b}} \frac{I_{b, 0}}{4 U_{0}}\right)^{1 / 3}[\mathrm{~dB}] .
$$

Using eq. (4.1) we calculate the small-signal gain per wavelength $G_{\lambda}$ for the different transverse photonic crystal modes, as a function of the number of electron beams. Figure 4.7 shows the results as data points connected with lines. Each curve corresponds to the small-signal gain of a certain photonic crystal mode. The figure shows that the highest small-signal gain is always found for mode 1, although the small-signal gain increases for each mode with the number of pump beams. Thus, as we have assumed mono-energetic electron beams a single wave number will grow and it is expected from eq. (4.1) that eventually a single mode dominates. Indeed, we observe this in the PIC calculations. By far the main power of more than $95 \%$ is contained in a single mode and only a minor fraction of less than $5 \%$ is distributed over all other modes at steady-state. This residual power found in the other modes might be caused by weak inhomogeneous gain broadening, 


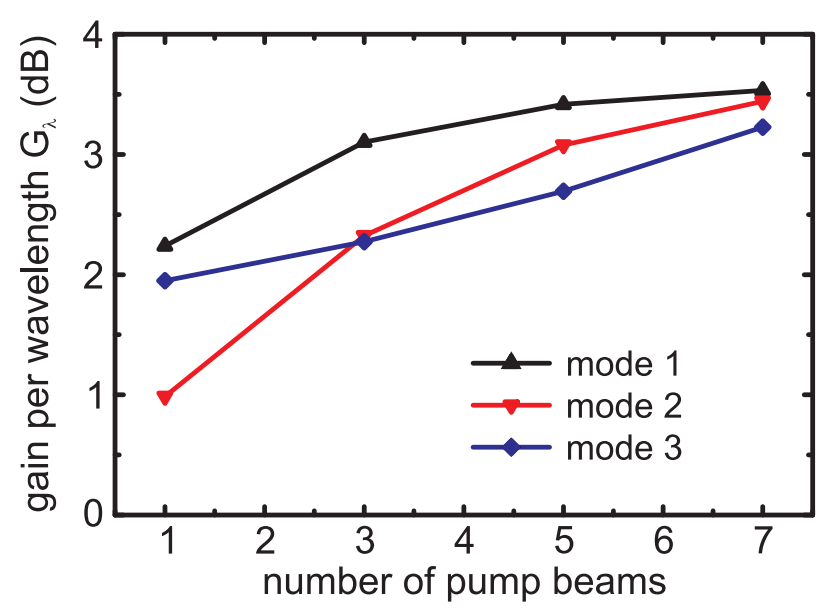

Figure 4.5: Small-signal gain per wavelength versus the number of electron beams pumping the pFEL of Fig. 4.1 at the velocity-matched wave number $k_{z, P h C}$. (Data points are connected with lines for a better visualization.)

which occurs if a sufficiently large velocity spread is present in the electron beam [197]. Indeed, this is also the case for our pFEL. We recall that, if mono-energetic beams are injected into the pFEL, this does not mean that the beams also remain mono-energetic inside the photonic crystal. As we discussed at the case of a pFEL pumped with a single beam, the kinetic energy of the electrons becomes slightly decreased upon injection into the crystal. This decrease depends on the precise transverse and longitudinal position of the electrons. A small energy spread of about $1.5 \%$ is found in the beam, which might cause a residual fraction of higher-order mode oscillation.

While the difference in gain for the modes and the presence of homogeneous gain broadening of the beam would explain that mode 1 dominates, it is not obvious to which effect or, more specifically, to which variables in eq. (4.1) this is to be addressed. For a closer inspection, let us discuss the various parameters in eq. (4.1) that determine the small-signal gain per wavelength $G_{\lambda}$. The beam current $I_{b, 0}$, the kinetic energy of the electrons expressed as acceleration voltage $U_{0}$ and the beam cross-section $S_{b}$ are constants and can be excluded from the discussion. To enable the comparison between the different modes, the total energy per unit cell $W$ has been normalized to $1 \mathrm{~J}$ throughout the calculations. Note that, this does not change the gain as only the field strength relative to the energy per unit cell enters eq. (4.1). We find from Fig. 4.2b that also the velocitymatched wave number $k_{z, P h C}$ is basically the same, as it changes less than $1 \%$ for the three modes. Also the group velocity, $v_{g}$, is hardly different for the modes, which we can also see by inspecting Fig. $4.2 \mathrm{~b}$, as the group velocity is the derivative of the dispersion. Thus, the difference in gain between the modes is mostly to be attributed to the difference 


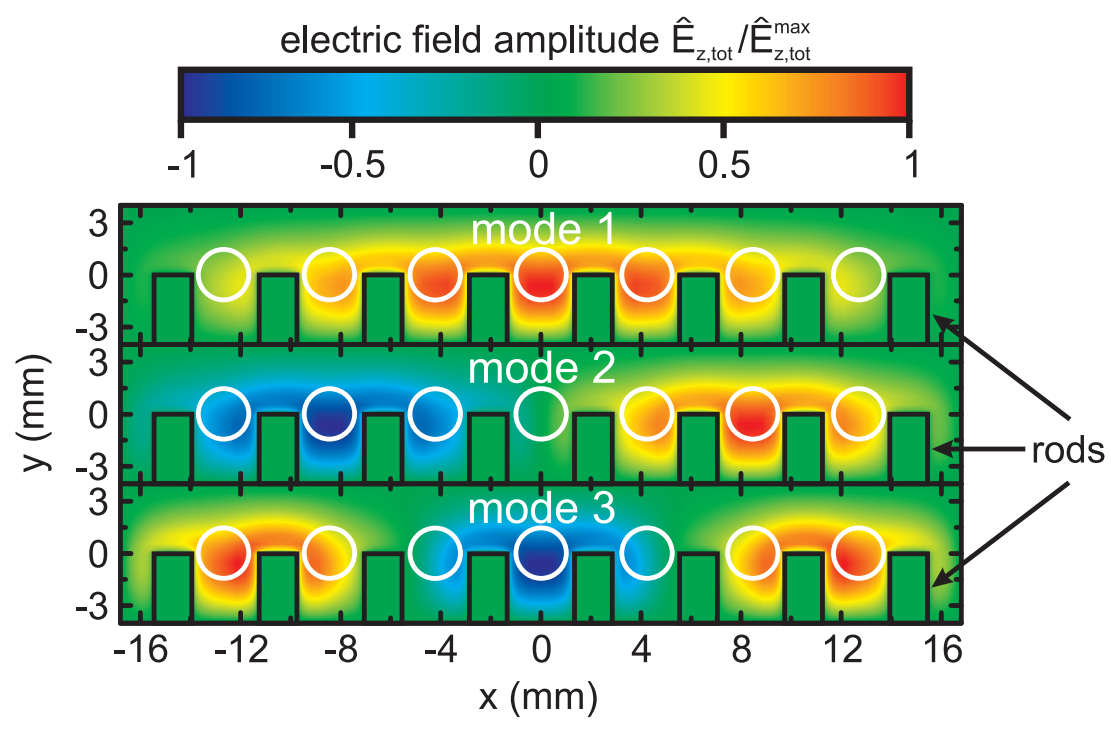

Figure 4.6: Total longitudinal electric field component $\hat{E}_{z, \text { tot }}$ at a cross section (xyplane) through the center of the rods. The frequencies for the modes are $\nu=15.87 \mathrm{GHz}$, $\nu=15.95 \mathrm{GHz}$ and $\nu=16.03 \mathrm{GHz}$. All three modes of the photonic crystal slab have the same wave number in the $0^{\text {th }}$ spatial harmonic of $k_{z, 0}=-873 \mathrm{~m}^{-1}$. The white circles indicate the edge of the electron beams and the rod positions are outlined via black rectangles.

in the field-electron beam overlap integral:

$$
\sum_{b=1}^{t} \int_{S_{b}}\left|E_{z, m}\left(x, y, k_{0}\right)\right|^{2} \mathrm{~d} \mathbf{S}_{b}
$$

Here, $E_{z, m}$, is the velocity-matched spatial harmonic amplitude in the considered photonic crystal mode.

To underline the meaning of this velocity-matched spatial harmonic amplitude $E_{z, m}$, let us recall the mathematical form of the longitudinal component $\hat{E}_{z, t o t}$ of a photonic crystal mode, eq. (2.23):

$$
\begin{aligned}
& \hat{E}_{z, t o t}\left(x, y, z, k_{z, 0}\right)=\sum_{m=-\infty}^{\infty} E_{z, m}\left(x, y, k_{z, 0}\right) \exp \left(-i k_{z, m} z\right) \\
& \text { with } k_{z, m}=k_{z, 0}+\frac{2 \pi}{a_{z}} m,
\end{aligned}
$$

This equation states that, when one knows the specific spatial longitudinal electric field distribution along the electron beam, one finds $E_{z, m}$ via a Fourier-transform. For a more detailed explanation and the precise numerical implementation the reader is referred to van Dijk [166]. This means that only a fraction of the total field $\hat{E}_{z, \text { tot }}$ is responsible 


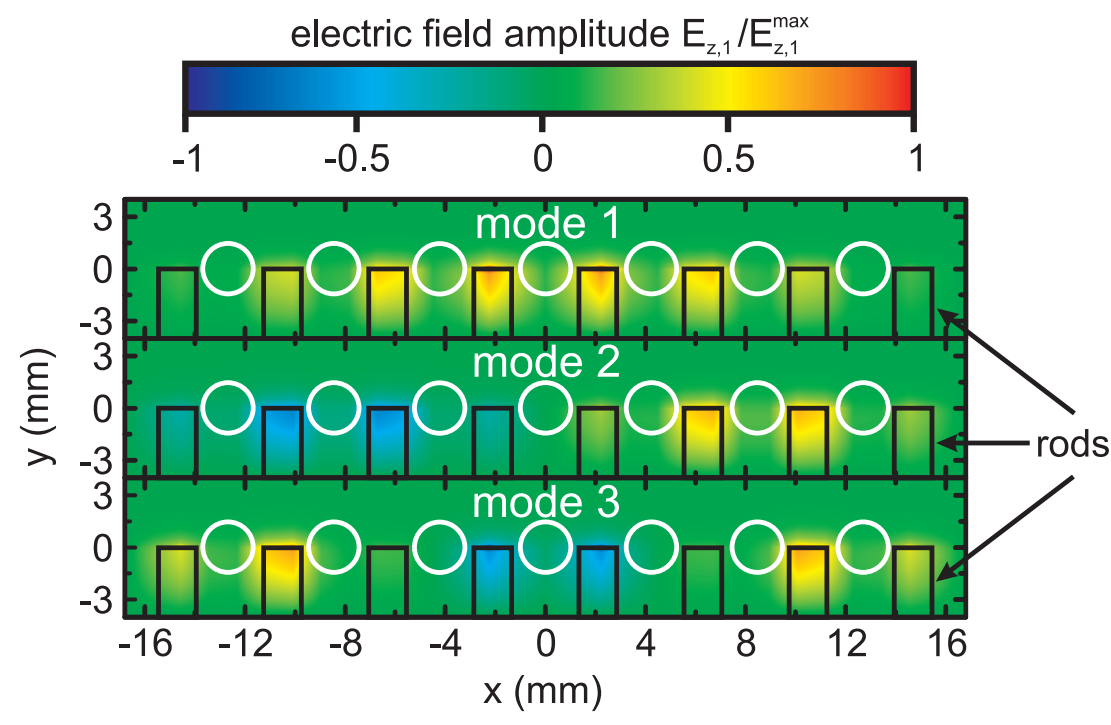

Figure 4.7: Spatial overlap between velocity-matched spatial harmonics amplitude $E_{z, 1}\left(x, y, k_{z, 0}\right)$ at $k_{z, 1}=1641 \mathrm{~m}^{-1}$ and electron beams for mode 1 , mode 2 and mode 3 of the photonic crystal slab. The corresponding frequencies for the amplitudes are $\nu=15.87 \mathrm{GHz}, \nu=15.95 \mathrm{GHz}$ and $\nu=16.03 \mathrm{GHz}$. The spatial harmonic amplitude $E_{z, 1}\left(x, y, k_{z, 0}\right)$ is plotted in a false color scale versus the transverse coordinates $x$ and $y$. The white circles indicate the edge of the electron beams and the rod positions are outlined via black rectangles.

for the small-signal gain. This fraction of the total field is the spatial harmonic with a wave number $k_{z, m}$ equal to $k_{z, P h C}$. To make this difference clear, let us compare the total longitudinal electric field component and the spatial harmonic with the wave number $k_{z, P h C}$ also graphically. The total longitudinal electric field component is what is obtained with an eigenmode solver when computing the dispersion from the unit cell of the crystal. This total longitudinal electric field component, $\hat{E}_{z, t o t}$, is shown in Fig. 4.6 as a function of the transverse coordinates $(x, y)$ in a cross-section through the center of the rods for the three modes considered. Each sub-figure shows the field distribution for the same wave number of $k_{z, 0}=-873 \mathrm{~m}^{-1}$ which corresponds to the operating frequency of mode 1 . It is clearly observed that the photonic crystal modes are distinguishable via the number of nodes in the $x$-direction. Further, the maximum field amplitude is found in-between the rods and the field is zero inside the rods.

Figure 4.7 shows the velocity-matched spatial harmonic amplitude of these three modes. Thus, the spatial harmonic number is $m=1$ and the associated wave number is $k_{z, P h C}=1641 \mathrm{~m}^{-1}$ (see. Fig. 4.2b). Of course, the same number and location of field nodes are observed, but the velocity-matched field amplitude is non-zero also at the transverse location $(x, y)$ of the rods. In fact for all modes the velocity-matched spatial 
(a) mode 2 pumping

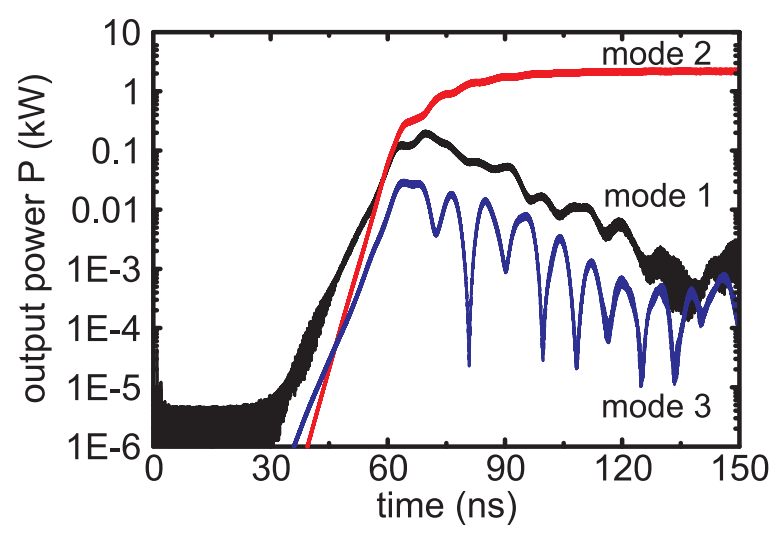

(b) mode 3 pumping

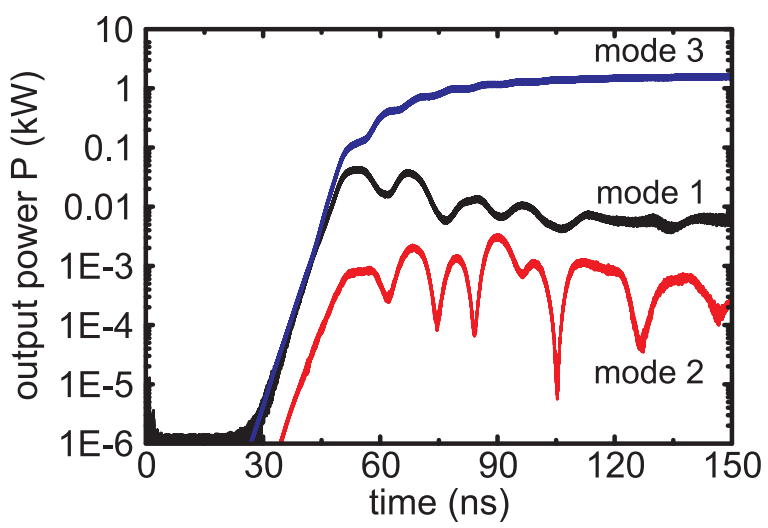

Figure 4.8: Dynamical growth of the output power for mode selective pumping schemes. (a) Power when the pFEL is pumped by only the beams $3 \mathrm{a}$ and $3 \mathrm{~b}$ to selectively excite mode 2. (b) Power when the pFEL is pumped by only the beams $4 \mathrm{a}$ and $4 \mathrm{~b}$ to selectively excite mode 3 .

harmonic amplitude $E_{z, 1}$ shows a local maximum at the transverse locations of the rods. Note that this is not in contradiction with the fact that the electromagnetic field inside a perfect conductor is zero [124]. None of the spatial harmonic amplitudes in the Fourier series needs separately to be zero inside the rods, as long as the total (phased) superposition of the spatial harmonics is zero inside the rods, which is indeed the case here (see Fig. 4.6).

The different small-signal gain of the various photonic crystal modes can now be understood by looking at the overlap of the velocity-matched spatial harmonic amplitude with the pumping electron beams. From Fig. 4.7 we observe that, for mode 1 of the photonic crystal slab, the overlap with the first spatial harmonic is highest for electron beam 1, as the overall envelope decreases towards the sidewalls of the waveguide. Thus, the highest contribution of the various beams to the small-signal of mode 1 is from beam 1. The envelope of the first spatial harmonic also exhibits no nodes inside the waveguide, as is the case for the total longitudinal electric field component shown in Fig. 4.6. Mode 2 possesses one node exactly at the center of the waveguide where the electron beam 1 is located. Similarly, mode 3 has two nodes at $x=-5.6 \mathrm{~mm}$ and $x=5.6 \mathrm{~mm}$, thus again close to where electron beams stream, (2a and $2 \mathrm{~b})$. This effect reduces for the higherorder modes the overlap with the pumping electron beams which is the reason, why the small-signal gain of these modes is lower than that of mode 1 .

As a direct test of the previous explanation for mode selection, we try to enforce the output being dominated either by mode 2 or mode 3 . According to the principle of best overlap with the electron beams, we chose the location of the beams such that the overlap 
of mode 2 or mode 3 is bigger than for all other modes. Pumping only with the beams $3 \mathrm{a}$ and $3 \mathrm{~b}$ should let mode 2 dominate. Pumping only by the beams $4 \mathrm{a}$ and $4 \mathrm{~b}$ should make mode 3 dominate. Figure 4.8 shows the growth of the strongest modes vs. time at the output port calculated for the two pump configurations. Initially, other modes seem to have a bigger power than mode 2 and mode 3 , respectively. This is due to different initial noise conditions for the modes. In our calculations different noise levels can be caused by different spontaneous emission powers for the various modes and, unfortunately, also by numerical effects. However, it can be seen that the exponential growth is strongest for mode 2 or mode 3 , respectively. After some time these modes become dominant about $60 \mathrm{~ns}$ for mode 2 in Fig. $4.8 \mathrm{a}$ and about $45 \mathrm{~ns}$ for mode 3 . Thereafter, the results show indeed that "The winner takes it all". The power in the other modes reduces to more than two orders of magnitude below the power of mode 2 and mode 3 , respectively. The favorable overlap with the pump beams leads to a spatial condensation via gain competition. This shows again that achieving single-mode oscillation is due to a spatial gain competition similar to homogeneous gain broadening in lasers.

This observation is an important finding for power up-scaling of pFELs without losing single-mode operation. Always one of the modes will have the best overlap with the electron beams. When supplying mono-energetic pump beams this should lead to a steady-state power dominated by the mode with the best field-electron overlap. Preferably, one adjusts the location of the pump electron beams and the transverse field nodes via the photonic crystal slab geometry, such that the lowest order mode dominates. Eventually, this might not be possible anymore, however, as long as the output is concentrated in a single transverse mode and a single longitudinal mode it could be converted into a single frequency $\mathrm{TEM}_{00}$ mode in free-space using suitable diffractive optical elements. This offers the maximum possible brightness when up-scaling the power via pumping a larger volume.

\subsection{Power scaling for multiple beams}

We have shown that, by increasing the pump power in a specific pFEL, it is possible to increase power and brightness, simultaneously. However, so far we have postponed a more detailed investigation of the powers that can be achieved in mode 1 , as found in Fig. 4.3. In this section we attempt to deduce a general power scaling law from the PIC calculations. Such power scaling law would be very desirable for an improved understanding of the underlying physics, but also for predicting the expected output power when injecting many - possibly hundreds to thousands - electron beams into photonic 


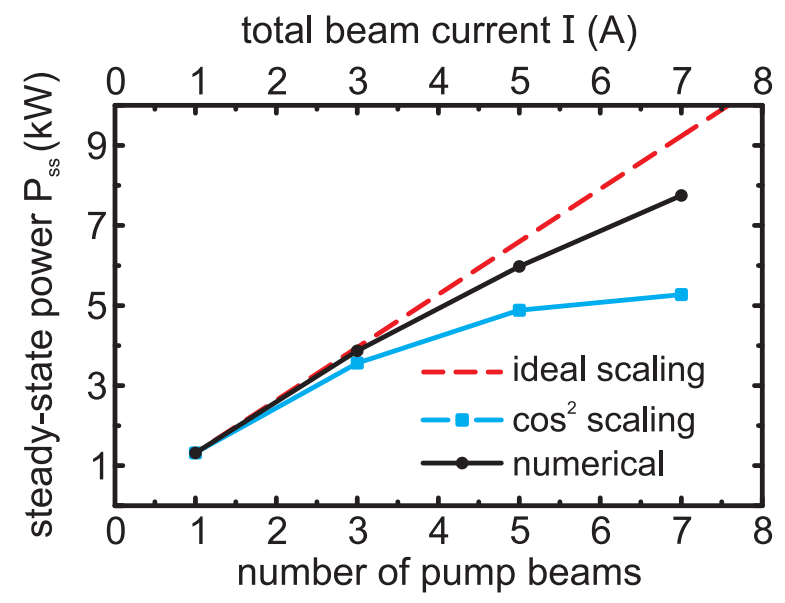

Figure 4.9: Steady-state output power versus the number of electron beams and total pump current. The numerical data is calculated via PIC calculations for the pFEL shown in Fig. 4.1 The power scaling is compared with two estimates. First, with assuming an infinitely wide photonic crystal where each beam contributes equally to the power (ideal scaling). The second estimate describes the photonic crystal slab as a dielectric-filled rectangular waveguide and assumes interaction with the lowest transverse TM-eigenmode, which has a cosine shaped field distribution along the transverse axes ( $\cos ^{2}$-scaling).

crystals. PIC calculations are most likely incapable to predict such power scaling in a reasonable amount of computation time because the modeling effort becomes incredibly excessive.

In order to identify a law for a general power up-scaling, Fig. 4.9 summarizes the steady-state power obtained with up to seven beams as found in Figs. 4.3 a-d. It is observed that clearly the output power increases with the number of pump beams. Thus, the output power also increases with the total pump power. However, for each consecutive increment of pump power, the increment in output power becomes smaller. This might indicate that a further power scaling is impossible. In order to find out if this is the case we compare the found power scaling to scaling laws found in literature.

The most commonly found power up-scaling in lasers is a linear increase with the pump power [169]. Indeed, this has been found with a single pump beam as seen in Fig. 3.8. Here, this most naive expectation would mean that, if one beam generates a output power $P_{1}$ at steady-state, $t$ beams should ideally generate a power $t P_{1}$. Figure 4.9 shows that such ideal scaling clearly overestimates the power up-scaling for the specific pFEL we have investigated.

To understand this discrepancy, note that the assumption that each beam contributes the same power to the total output implies that each electron beam would contribute with the same strength to the pumping of the electromagnetic field of the dominating mode. 
However, for transversely finite structures, including the pFEL we study here, the metal boundaries enforce a transverse variation of the overall electromagnetic field envelope. The reason for this is that the electric field tangential to the metallic side walls has to be zero, see Fig. 4.6. As a result the electron beams that are located closer to the wall of the waveguide interact with a weaker electromagnetic field than the beams in the center of the waveguide. This reduces the small-signal gain contribution of the beams near the walls, and it is reasonable that this also reduces their contribution to the the steady-state output power.

To relate the electromagnetic field strength at the beam location to the steady-state power, let us compare the pFEL to a hypothetical slow-wave FEL. This FEL achieves velocity-matching via a rectangular waveguide completely filled with a dielectric with a refractive index $n$ through which the electrons can nevertheless propagate. Thereby, we approximate the photonic crystal by a homogeneous effective dielectric medium. In this case, the first transverse mode with non-zero $E_{z}$ field is the $\mathrm{TM}_{11}$ waveguide mode [165]. This mode has a cosine shaped field profile along the transverse directions $x$ and $y$. The $E_{z}$ field distribution has nodes at the metallic side-walls and the field maximum lies at the center of the waveguide.

The steady-state output of these so-called Čerenkov lasers has been studied analytically by Asgekar in a one-dimensional model [76]. In analogy to solid-state lasers he defines a saturation intensity, $I_{\text {sat }}$, or equivalently a saturation power $P_{\text {sat }}$, as follows.

$$
P_{\text {sat }}=\frac{1}{2} \epsilon_{0} \frac{m_{\text {el }}^{2}}{e^{2}} \frac{v^{5} \gamma^{6}}{L^{4}} \lambda_{\text {matched }}^{2} S
$$

Here $S$ is the transverse cross section of the electron beam and $\lambda_{\text {matched }}=2 \pi k_{\text {matched }}^{-1}$ is the velocity-matched wavelength in a Čerenkov laser. The saturation power describes at which power the gain reduces to half of its small-signal value due to gain saturation. Asgekar's analysis is based on the assumption that, at that power, electrons have on average slipped backwards with respect to the electromagnetic wave by a certain phase $\Omega$, where the value of $\Omega$ is taken from numerical calculations. Further, he shows that the gain coefficient, $g_{0}$, and the saturation power $P_{\text {sat }}$ are related to the electron beam power ${ }^{1}$

$$
4 N \gamma^{2} P_{\text {sat }} g_{0}=\frac{v^{2}}{c^{2}} P_{\text {beam }}
$$

\footnotetext{
${ }^{1}$ Please, note that the original paper has a typographic error for formula (4.6), which is in the paper formula (16). The saturation intensity $I_{\text {sat }}$ should be the saturation power $P_{\text {sat }}$ if one follows its derivation. This is also easily observed by performing a simple dimensional analysis of both sides of the formula.
} 
Here $g_{0}$ relates in our notation to

$$
g_{0}=8 \pi^{2} C^{3} N^{3}
$$

$P_{\text {beam }}$ is the kinetic beam power which yields for the case of a non-relativistic, low-energy beam, where $\gamma$ can be set to unity,

$$
P_{\text {beam }}=U_{0} I_{0}
$$

In the following, we use eq. (4.6) for approximating the steady-state output power $P_{s s}$. For that, we assume that the efficiency defined as

$$
\eta=\frac{P_{s s}}{P_{\text {beam }}}
$$

is to a first order approximation, not influenced by the current streaming through the crystal. Indeed, this has also been found in the calculations for the single-beam pumped pFEL (see Fig. 3.8). Thus, in this one-dimensional theory the following relation can be found for the steady-state power $P_{s s}$, when combing eqs. (4.5) to (4.9)

$$
P_{s s}=4 \epsilon_{0} \eta k_{\text {matched }}^{2} v^{3} c^{2} \frac{m_{\text {el }}^{2}}{e^{2}} C^{3} S \text {. }
$$

In this one-dimensional theory it is clearly seen that the crucial parameter to determine the steady-sate power is the gain parameter $C$. However, for applying eq. (4.10) to our hypothetical Čerenkov FEL, we need to incorporate transverse variations into the model.

For this, we assume that all of the beams we use are contained in a single, super electron beam with a total beam current $I_{0}=t I_{b, 0}$. This beam current increases then linear with the number of beams $t$. The saturation power of this super beam is assumed to be approximately given by eq. (4.5), as it is found that the saturation power is completely independent of the beam current. Transverse variations are then introduced into this model via the small-signal gain coefficient $C$ that can for $t$ beams be written as [200]:

$$
C=\left(\frac{1}{t} \frac{\sum_{b=1}^{t}\left|E_{z, \text { matched }}\left(x_{b}, y_{b}, k_{\text {matched }}\right)\right|^{2}}{2 k_{\text {matched }}^{2} W v_{g} S_{b}} \frac{I_{b, 0}}{4 U_{0}}\right)^{1 / 3} .
$$

Here, we have further used that the variation of the electric field around the $b^{\text {th }}$ beam, can be roughly approximated by the electric field at the center of that beam $\left(x_{b}, y_{b}\right)$. Specifically, for the maximum amount of seven beams, i.e., $t=7$ the velocity-matched longitudinal electromagnetic field component, becomes the following in our hypothetical 
Čerenkov laser.

$$
\begin{array}{r}
\sum_{b=1}^{t}\left|E_{z, \text { matched }}\left(x_{b}, y_{b}, k_{\text {matched }}\right)\right|^{2}=\left|E_{z, \text { matched }}\left(0,0, k_{\text {matched }}\right)\right|^{2} \\
\times\left[\cos ^{2}(0)+2 \cos ^{2}\left(1 a_{x} / 8 a_{x} \pi\right)+2 \cos ^{2}\left(2 a_{x} / 8_{a} x \pi\right)+2 \cos ^{2}\left(3 a_{x} / 8 a_{x} \pi\right)\right]
\end{array}
$$

This expression contains the cosine-shaped variation of the considered $\mathrm{TM}_{11}$ waveguide mode in the laser. It is further assumed that, by increasing the number of beams, the electromagnetic field that effectively bunches the super electron beam is the square of the quadratic mean value at the location of the electron beams in our hypothetical Cerenkov FEL. Using the gain parameter $C$, eq. (4.11), in eq. (4.10) we find as an approximation for the steady-state power, that

$$
P_{s s}=2 \epsilon_{0} \eta \frac{n^{2}}{W} I_{b, 0} U_{0} \frac{1}{t} \sum_{b=1}^{t}\left|E_{z, \text { matched }}\left(x_{b}, y_{b}, k_{\text {matched }}\right)\right|^{2} .
$$

We observe indeed that the above derivation predicts a linear scaling with $I_{b, 0}$ and $U_{0}$ if the longitudinal field strength present at each beam location is the same, as expected from Fig. 3.8. However, for a cosine shaped field distribution the square of the quadratic mean value is smaller than for a transversally homogeneous field. Therefore, we can expect the resulting power scaling to be less than linear. Figure 4.9 shows the calculated power scaling four our hypothetical Čerenkov FEL, when we set that the power emitted by a single electron beam is equal to our pFEL pumped with a single electron beam. The figure shows that the power increment reduces with increasing number of pump beams, as each added pair of adjacent pump beams interacts with a smaller longitudinal electric field than the center beam. While this power scaling confirms the principal result, of a reduced power increment with each added pair of adjacent pump beams, the figure also shows that the absolute agreement to our numerically calculated data points is not better than for a linear scaling. We suspect that this is due to our coarse approximations via an effective dielectric medium which does not accurately model the fine structure of the field distribution in a photonic crystal. Nevertheless, there is an overall correct tendency of the $\cos ^{2}$-weighted power scaling with the numerical results. This indicates that the varying field strength at the beam locations influences the shape of the power scaling law.

In conclusion, we found that the transverse variation of the photonic crystal mode affects the steady-state output power. While this means the power up-scaling with the number of beams is slightly less than linear, an overall power scaling with the number of beams is still expected. 


\subsection{Conclusion}

In conclusion, we presented how a pFEL with a large pump volume can be used to achieve a single-mode power up-scaling, by pumping it with many electron beams. In other lasers, it is typically observed that increasing the pump volume, leads to multi-mode oscillation [95, 101, 192-196]. However, when calculating the output for a specific pFEL, the lowest transverse mode remains dominating and contains more than $95 \%$ of the total power at a single frequency.

We found that this is likely due to the circumstance that a mono-energetic electron beam can be considered as providing mode competition for the gain in analogy to homogeneous gain broadening. In this competition, the mode with the highest small-signal gain eventually suppresses the amplification of other modes by depleting their gain. The smallsignal gain of the various potentially oscillating modes depends on the overlap between the velocity-matched spatial harmonic amplitude and the electron beams. Indeed, we found that the spatial overlap between the dominant mode and the electron beams is higher compared to other modes. When increasing the transverse width of the photonic crystal slab, a certain transverse mode will always have the best overall overlap with the pump beams. Even if this is not the lowest transverse mode, the output remains concentrated in a single transverse mode and a single longitudinal mode which can be converted into a single frequency $\mathrm{TEM}_{00}$ mode in free-space using suitable diffractive optical elements achieving the highest possible brightness for a given power and frequency.

To devise a power scaling law we discussed the trend of the power increment versus the number of electron beams. Each pump power increment via adding more beams results in a slightly less output power increment. With seven beams a maximum output power of $8 \mathrm{~kW}$ in a single-mode is found for the laser. This power is slightly less than is expected for a linear scaling with the pump power. We investigated whether such deviation is due to the varying electric field strength along the transverse direction. The power scaling law found with this assumption provides a similar tendency as found in the PIC calculations.

The power scaling discussion is of central importance for a frequency scaling of pFELs, as we discussed in the introduction of this chapter, especially, as the increased power output is found in a single-mode. For example, given the power scalability pointed out at a particular example, pFELs might fill in the so-called THz gap. The name THz-gap expresses the lack of higher-average power THz sources $(P>1 \mathrm{~W})$ that, at the same time, can be compact in size $[64,65,201]$. For instance, backward-wave oscillators and traveling wave tubes may be constructed with dimensions that provide output in the $\mathrm{THz}$ range, but their maximum output is not more than a few tens of milliwatts [65, 202, 203]. The strongest of these sources use extremely high current densities of more than $150 \mathrm{~A} / \mathrm{cm}^{2}$ 
[203]. These densities tremendously complicate the electron beam generation and guiding. Using our concept of scaling, i.e., by dividing the total electron current over a large volume, in the form of many electron beams, avoids such problems. By extending the number of crystal periods in a transverse direction, here the $x$-direction, and possibly also the $y$-direction, photonic crystals naturally provide an increasing number of vacuum channels for the propagation of multiple electron beams. We envision that eventually the current might be distributed over hundreds or thousands of electrons beams generated, e.g., by field-emitter arrays [187] to pump a single-mode of a pFEL at THz frequencies and generate several Watts of output power. 



\section{Mapping electromagnetic field components inside photonic crystals}

\subsection{Introduction}

Photonic crystals provide spontaneous and stimulated emission in a pFEL by offering a suitable spatio-temporal distribution of the electromagnetic field modes. By shaping the electromagnetic field, such that a velocity-matched and longitudinally oriented $E_{z}$ field is present, photonic crystals enable bunching of the electron beam which eventually leads to laser oscillations. Besides pFELs, there are a wide range of other fundamental experiments that rely on proper shaping of the electromagnetic field in a photonic crystal to control the interaction of light and matter. For example, spontaneous emission from quantum dots is enhanced or inhibited by placing them inside a photonic crystal [19, 20, 24, 25, $28,29]$. Such experiments also have far reaching technological impact in, for example, the development of efficient micro-scale lasers, LEDs or solar cells [30-38, 204-206].

All these scientific examples and applications are enabled by the particular field distribution present inside the photonic crystal, i.e., at the locations of the free electrons or the embedded light sources. Eigenmode fields of ideal photonic crystals, i.e., assuming a perfect periodicity, can be calculated by numerical methods such as eigenmode or 
FDTD methods [121, 141, 207, 208]. However, all real photonic crystals inevitably suffer from unpredictable non-periodic local deviations due to both fabrication errors and, also fundamentally, due to thermodynamical arguments $[209,210]$. Such deviations are very challenging to include into numerical calculation and require certain idealizations of the structure geometry even when sophisticated methods are applied [211, 212]. A measurement is the only way to analyze the electromagnetic field inside a real photonic crystal. However, a method to measure the absolute field strength of an eigenmode inside a real photonic crystal has never been published.

To date the most commonly used method to map local fields of photonic crystals has been near-field scanning optical microscopy (NSOM) [213-231]. This technique relies on scanning a small tip of a tapered optical fiber above the surface of a photonic crystal that collects part of the evanescent field with subwavelength resolution. However, while this method has been used to measure individual field components $[226,228,232]$ it is restricted to probing local fields outside the crystal near its surfaces while the field deep inside the crystal cannot be mapped. If the probe of an NSOM is placed inside the photonic crystal it would distort the fields beyond recognition, due to its large size compared to the photonic crystal features, see e.g., [222]. Thus, one is forced to make assumptions about the internal scattering of the fields under study to the detected evanescent fields. In case of three-dimensional crystals, relating the fields at the surface to the fields in the bulk is even more challenging [221] than in the widely studied two-dimensional slab systems. What is desirable is to devise a method that can probe the absolute strength of the electromagnetic field inside a photonic crystal.

While non-periodic deviations inside photonic crystals are especially important at $\mathrm{THz}$ or optical frequencies [233, 234], non-periodic deviations should be a minor concern at microwave frequencies. It is possible to precisely manufacture microwave structures, thus minimizing such deviations. However, for the particular example of microwave photonic crystals for application in a pFEL a method to precisely characterize the field distribution inside the photonic crystal remains important. During laser operation the vacuum pressure in the laser needs to be typically below $1 \cdot 10^{-7}$ mbar. Such pressures are required to allow the operation of thermionic dispenser cathodes. Dispenser cathodes provide a high current, thus high gain, but they are very vulnerable to exposure to air, especially after first operation [235-238]. For example, the specific gun we intend to use for a first experimental demonstration should not be exposed to air more than a few times [239]. Hence, after the laser has been operated once, breaking the vacuum is to be prevented at all costs. Should the laser not operate as expected, the standard procedure is to investigate the proper working of each component individually to detect the problem. This 
requires opening the vacuum vessel and imposes a high risk of destruction to the cathode. Thus, it is desirable to thoroughly characterize the photonic crystal before evacuating the laser. As has been discussed in previous chapters for laser operation, the longitudinally oriented field strength is most important. As we pointed out before, such a method is not readily available and it would be desirable to devise such method.

Here we demonstrate a method to measure the absolute strength of the electromagnetic field distribution inside photonic crystals. Our method relies on measuring the resonant frequencies of standing waves in a photonic crystal of finite length that is enclosed by two mirrors. A subwavelength scatterer placed inside the crystal scatters the electromagnetic field which shifts the frequency of the resonances of the photonic crystal modes proportionally to the square of the electric and magnetic field strength at the scatterer position $[127,133,240,241]$. By measuring the frequency shift as a function of the spatial position of the scatterer, we obtain maps of the field strength versus position. To demonstrate the method we perform measurements using a typical photonic crystal as intended for the use in pFELs. In order to maximize gain such photonic crystals are designed such that the longitudinal field component dominates throughout most of the inside of the photonic crystal slab. This allows the use of a spherical, metallic bead as a scatterer to map the dominant longitudinal electric field component inside the photonic crystal slab.

\subsection{Measurement method}

To map the electromagnetic field, the photonic crystal is placed between two mirrors. The mirrors restrict the electromagnetic field in the photonic crystal to discrete longitudinal photonic crystal modes with associated resonance frequencies $\nu_{0}$ and wave numbers $k_{z}$. To measure the electromagnetic field we place a scatterer inside the photonic crystal and measure the shift of the resonance frequencies. We chose a scatterer in the Rayleigh regime with a small size parameter $x \ll 1$, with $x=2 \pi R / \lambda$. Here, $R$ is the radius of the object and $\lambda$ the wavelength. In this regime the field is approximately constant throughout the scatterer volume. Thus, the scattering can be treated within the electrostatic approximation to calculate the resulting frequency shift $\Delta \nu$ due to the scatterer. Using a spherical metallic scatterer with a radius $R$ we obtain [127, 240, 241].

$$
\Delta \nu(\mathbf{r})=\frac{\frac{1}{2} \mu_{0} \hat{\mathbf{H}}(\mathbf{r})^{2}-\epsilon_{0} \hat{\mathbf{E}}(\mathbf{r})^{2}}{U} \pi R^{3} \nu_{0}
$$

where $\hat{\mathbf{E}}(\mathbf{r})$ and $\hat{\mathbf{H}}(\mathbf{r})$ are the unperturbed electric and magnetic fields at the location $\mathbf{r}$ of the scatterer, respectively. $U$ is the total energy stored inside the unperturbed 
cavity, $\epsilon_{0}$ is the permittivity and $\mu_{0}$ the permeability of free space. If we measure the frequency shift versus the scatterer location $\Delta \nu(\mathbf{r})$ we will map, in a general photonic crystal, the electromagnetic field quantity $\left(\frac{1}{2} \mu_{0} \hat{\mathbf{H}}(\mathbf{r})^{2}-\epsilon_{0} \hat{\mathbf{E}}(\mathbf{r})^{2}\right)$. In certain photonic crystal geometries particular field components dominate, i.e., the field strength of a single component is much greater than for all other components. Measuring the total field is then effectively a measurement of this dominating field component.

In the photonic crystal of a pFEL it is most suitable to let the longitudinal field component dominate because this maximizes the amplification of the laser. In this case the frequency shift becomes

$$
\Delta \nu(\mathbf{r}) \approx-\frac{\epsilon_{0} \hat{E}_{z}(\mathbf{r})^{2}}{U} \pi R^{3} \nu_{0}
$$

Solving for the $\hat{E}_{z}$ component yields

$$
\hat{E}_{z}(\mathbf{r})=\sqrt{\frac{-\Delta \nu(\mathbf{r}) U}{\pi R^{3} \epsilon_{0} \nu_{0}}} .
$$

Equation (5.3) shows that it is possible to map the absolute strength of the $\hat{E}_{z}$ field component by measuring the frequency shift of the longitudinal resonances $\Delta \nu$ versus the bead position and by determining the total energy stored in the cavity $U$ for a specific input power $P_{i n}$.

\subsection{The photonic crystal slab}

In this section we present the photonic crystal slab that is used to demonstrate the method. The unit cell of the photonic crystal slab we use is shown in Fig. 5.1a. The unit cell has similar geometry as the crystals we studied in the chapters 3 and 4 . A rectangular lattice of metal rods is enclosed inside a rectangular metal waveguide causing

the $\hat{E}_{z}$ field component to dominate above all other field components inside the structure. However, the unit cell used is a supercell instead of a simple cell photonic crystal slab, which has been initially considered beneficial for use in pFELs and therefore the crystal was immediately available at the time we carried out the measurements. A rectangular lattice of metal rods with a central line defect at $x=0$ forms the basis of the supercell. The photonic crystal has a lattice constant of $a_{z}=7.5 \mathrm{~mm}$ along the $z$-direction and the lattice constant is $a_{x}=6.75 \mathrm{~mm}$ along the $x$-direction. The third transverse row of rods is missing inside the supercell. In total the supercell consists of 12 rods and has a length of $a_{z, \text { eff }}=22.5 \mathrm{~mm}$, as indicated in Fig. 5.1a. The diameter of the rods is $4 \mathrm{~mm}$ and the 
(a)

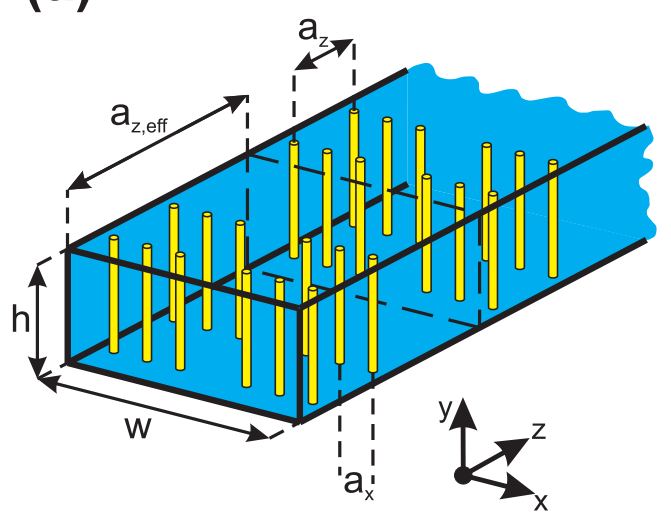

(b)

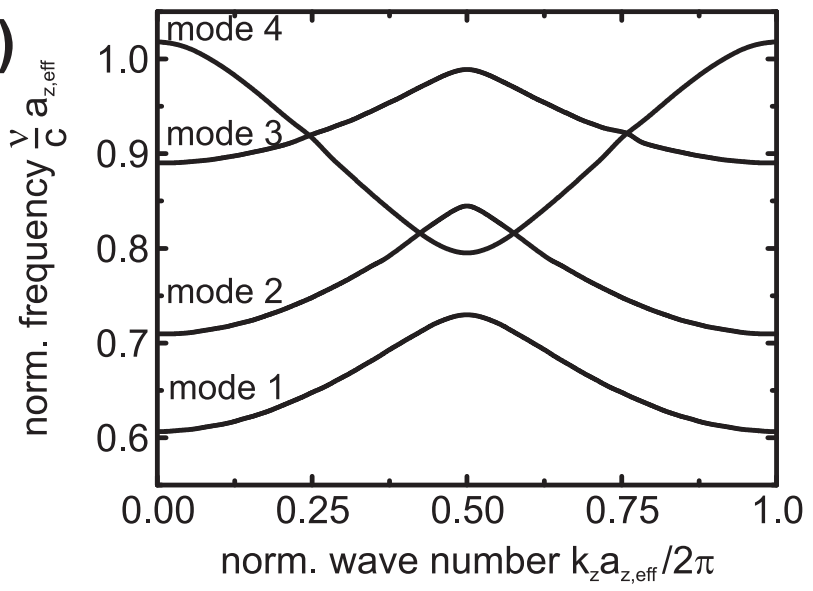

Figure 5.1: (a) Schematic three-dimensional view of the photonic crystal slab indicating the defining geometry parameters. Metallic rods are placed inside a rectangular metallic waveguide. The dashed line indicates the size of the supercell. (b) Calculated band structure of the photonic crystal slab showing the four lowest photonic crystal modes having a non-zero $E_{z}$ component of the electric field.

surrounding waveguide has a width of $w=47.25 \mathrm{~mm}$ and a height of $h=20 \mathrm{~mm}$.

To calculate the band structure of this photonic crystal slab, a finite-difference timedomain (FDTD) method is used [208]. In the calculations, through the application of appropriate periodic boundary conditions to the unit cell, the photonic crystal slab is taken to be infinitely long in the $z$-direction. For simplicity, all metal parts are treated as perfect electric conductors which is well justified in the microwave range. Figure 5.1b shows the results for the four lowest-frequency photonic crystal modes with a non-zero longitudinal electric field component. Due to the z-periodicity of the slab, the dispersion in the $0^{\text {th }}$ spatial harmonic, i.e., for normalized wave numbers $\left(a_{z \text {,eff }} k_{z}\right)$ between 0 and $2 \pi$, repeats with increasing wave number [44] and the finite transverse size of the waveguide results in a lowest allowed normalized frequency of 0.61 for mode 1 . No other photonic crystal modes exists below this cut-off frequency.

For a comparison with experimental field mapping data we calculate the local $\hat{E}_{z}$ field distribution of mode 1 and mode 2. The FDTD method is applied at the resonant frequency of mode 1 and 2 for a normalized wave number of $a_{z \text {,eff }} k_{z}=0$ which corresponds to a normalized frequency of 0.61 and 0.71 respectively. Figures 5.2a through $5.2 \mathrm{~d}$ show the $\hat{E}_{z}$ field pattern of the modes at two transverse planes at different $z$ coordinates. The first plane is taken through the center of the first row of rods at $z=0.5 a_{z}$ and the second plane is taken through the empty row of the unit cell at $z=2.5 a_{z}$ where mapping is performed. As expected, the field pattern is symmetric due to the symmetry of the photonic crystal slab. Furthermore, throughout all field patterns it is observed that mode 


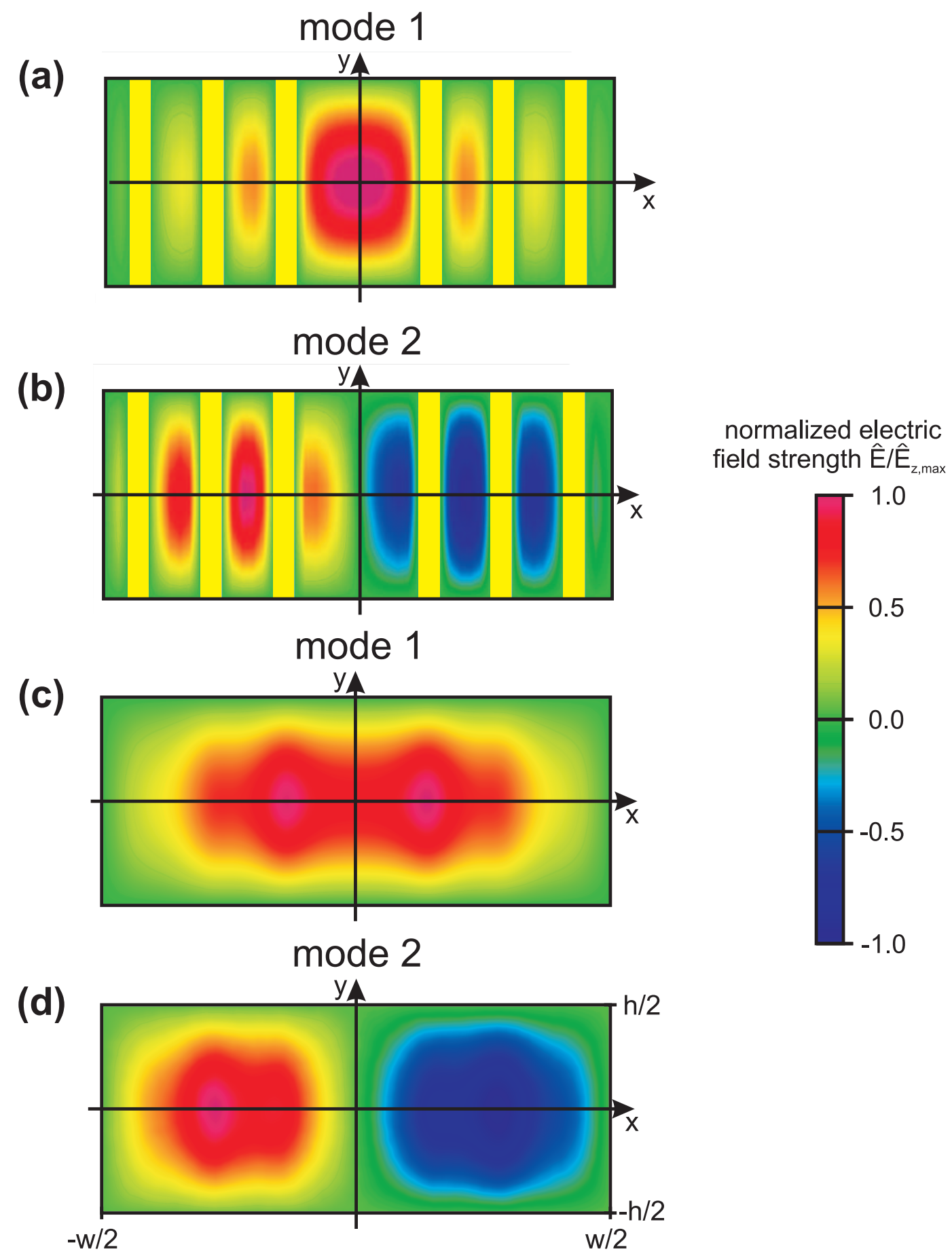

Figure 5.2: Transverse longitudinal electric field distribution, $\hat{E}_{z}$, of the photonic crystal slab for mode 1 and mode 2 at two cross sections ( $x y$-plane) inside the unit cell. First, through the first row of rods (at $z=0.5 a_{z}$, (a) and (b)). Second, at a cross section through the empty part of the waveguide (at $z=2.5 a_{z}$, (c) and (d)). The normalized wave number for both patterns is $k_{z} a_{z \text {,eff }}=0$ and the corresponding normalized frequency for (a) is 0.61 and for (b) 0.71 . 
2 has a field node at the center of the waveguide which will explain that mode 2 is not found in the experimental data later on.

\subsection{Experimental setup}

A schematic view of the experimental setup is shown in Fig. 5.3. The high-precision manufactured photonic crystal, whose properties have been described in section 5.3, is placed between two highly reflective alumimun mirrors to form a resonator. In the longitudinal direction, the resonator contains a total of 15 unit cells of the photonic crystal. The first mirror is positioned at a distance of $0.5 a_{z}$ from the center of the first row of rods and the second mirror is positioned at a distance of $1.5 a_{z}$ from the last row of rods.

The photonic crystal slab is fabricated as follows. A channel with a rectangular cross section of $47.25 \mathrm{~mm} \times 20 \mathrm{~mm}$ is milled into a solid aluminum block (bottom plate) and is covered with an aluminum top plate. Both plates contain holes for mounting the rods. The rods are hollow brass cylinders with an inner diameter of $2 \mathrm{~mm}$ and an outer diameter of $4 \mathrm{~mm}$. The rods are positioned with screws extending from the top plate through the rods and attach into the bottom plate. This also provides an electrical connection between the rods and the waveguide. The total positioning accuracy for the rods is estimated to be less than $100 \mu \mathrm{m}$, corresponding to a high accuracy of about $1.4 \%$ of the inter rod distances.

To measure the resonant frequencies of the photonic crystal slab cavity, a Hertzian dipole antenna [242] is mounted at the center of each mirror (see Fig. 5.3). Both antennas

point along the $z$-direction to excite or detect modes with a non-zero $\hat{E}_{z}$ field component. One antenna acts as an emitter - labeled "in" in Fig. 5.3 - while the other antenna acts as a receiver for transmission measurements - labeled "out" in Fig. 5.3. As a compromise between minimal loading of the resonator by the antennae and sufficient coupling to the modes, the length of both antennas was selected as $4 \mathrm{~mm}$. Note that, with the antenna position in the center of the mirror, modes with an $\hat{E}_{z}$ field node in the center cannot be excited, as is the case for mode 2 shown in Fig. 5.2b.

The other end of both antennae is connected to a network analyzer via coaxial SMA cables. The network analyzer is formed using a tunable microwave source with a maximum frequency of $20 \mathrm{GHz}$ (Wiltron, model 69147A), two directional couplers (Krytar, model 2610) and two microwave power meters (Anritsu, model ML1438A with power heads MA2444A and MA2424B). The network analyzer is calibrated before the measurements to compensate for frequency dependent losses in cables and other components. The accuracy is estimated to be about $10 \%$, as re-connecting coaxial cables typically has an effect of 


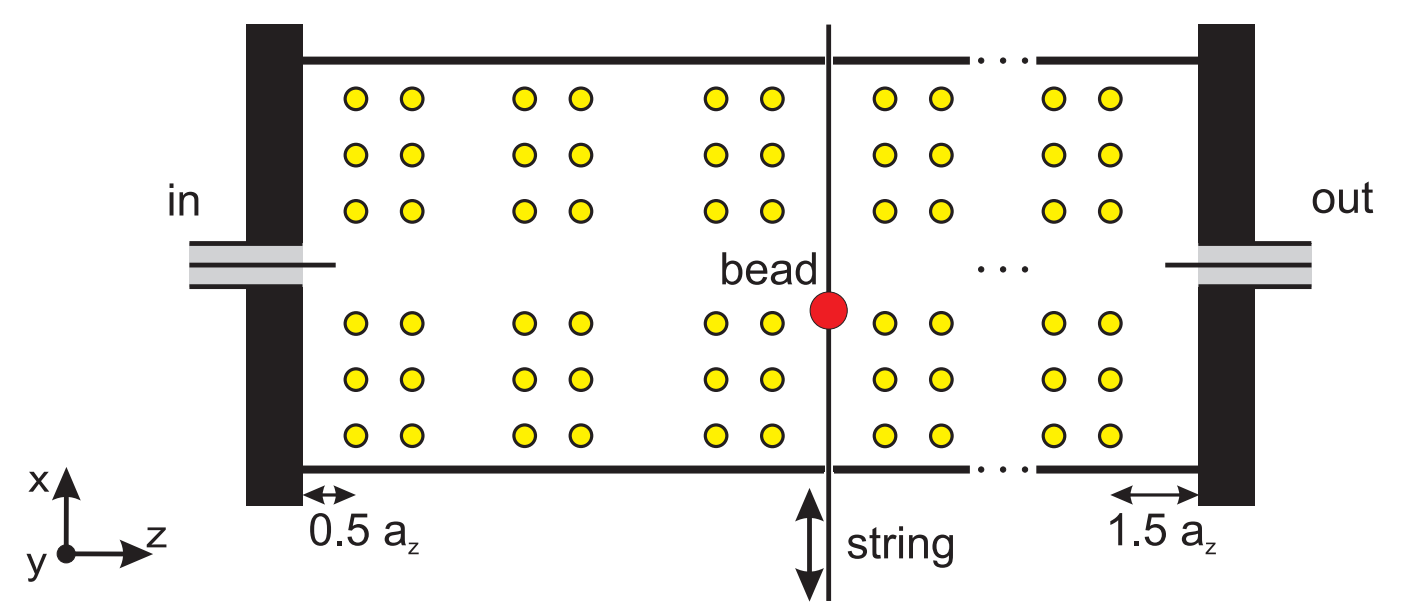

Figure 5.3: Schematic view of the setup to measure the electromagnetic field inside the photonic crystal slab by a scatterer. The figure shows a cross section through the photonic crystal slab at $y=0$. The photonic crystal slab is sandwiched between two aluminum mirrors (bold black). The input and output antennas are mounted at the center of both mirrors. To map the $\hat{E}_{z}$ field component along the $x$-direction a spherical metallic scatterer, which is mounted on a string, can be moved throughout the photonic crystal slab.

this magnitude. Using this setup, we can measure the reflection and transmission spectra of the photonic crystal slab cavity. For the measurements the frequency resolution is set to $250 \mathrm{kHz}$ and the input power $P_{\text {in }}$ from the network analyzer is $1 \mathrm{~mW}$.

To map the electromagnetic field inside the photonic crystal slab the scatterer is scanned through various locations inside the resonator. The scatterer is a stainless steel bead with a radius of $R=2 \mathrm{~mm}$ which sits on a $0.3 \mathrm{~mm}$ thick nylon string. As shown in Fig. 5.3 the string runs through two small holes $(300 \mu \mathrm{m})$ in the opposing side walls. The holes are positioned in the center of the side walls $(y=0)$ and at $z=2.5 a_{z}$ inside the 3rd unit cell, as counted from the input side. A $4 \mathrm{~mm}$ deep cylindrical cavity with a diameter of $4.5 \mathrm{~mm}$ is fabricated at the position of the holes in the inner surface of the side walls in which the bead can fit completely. One end of the string is attached to a weight to keep the string straight via tension, and the other end of the string is mounted on a translation stage. The translation stage is used to position the bead with a relative accuracy of better than $0.05 \mathrm{~mm}$. The absolute position is calibrated using the position at which the scatterer completely disappears within the cylindrical cavity. The error in this position is estimated to be smaller than $0.1 \mathrm{~mm}$. 


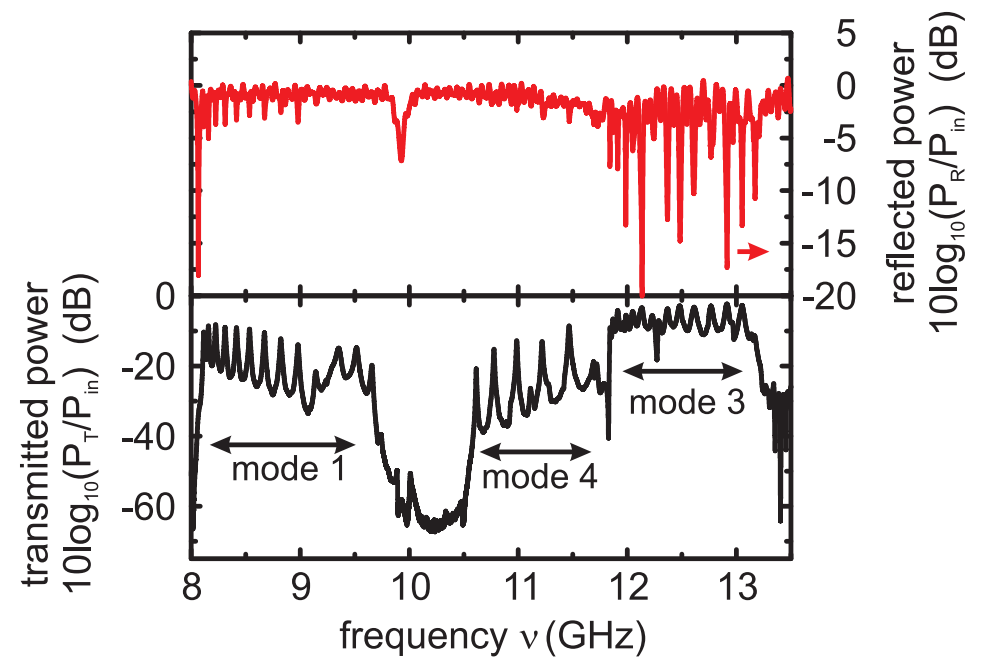

Figure 5.4: Transmission and reflection spectrum of the photonic crystal slab without a scatterer between $8.0 \mathrm{GHz}$ and $13.5 \mathrm{GHz}$. The peaks correspond to the various longitudinal modes for each transverse mode.

\subsection{Dispersion measurement}

Before measuring the $\hat{E}_{z}$-field component of the photonic crystal slab resonator we verified the appropriate description of the slab using the FDTD model. Specifically, to confirm the crystal dispersion, we measured the resonance frequencies of the different longitudinal and transverse modes without a scatterer inside the photonic crystal. Measuring the dispersion is based on determining the longitudinal resonance frequencies of a finite-length photonic crystal consisting of $n$ unit cells and assigning wave numbers to each observed resonance frequency [243]. For the assignment we consider the phase advance $\delta \phi$ of standing waves per round trip in the resonator. At each longitudinal resonance the phase advance per round trip along the $z$-direction is a multiple of $\pi$ :

$$
\delta \phi=n a_{z, \text { eff }} k_{z, 0}=m \pi \quad m=1,2 \ldots
$$

Here $n a_{z, \text { eff }}$ is the geometrical length of the resonator and $k_{z, 0}$ is the wave number in the $0^{\text {th }}$ spatial harmonic. As the resonator mirrors enclose 15 unit cells of the photonic crystal, $n=15$ longitudinal resonances with a finite wavelength are expected [243] having a normalized wave number $a_{z, \text { eff }} k_{z}$ of

$$
0<a_{z, \text { eff }} k_{z, 0}=\frac{m}{n} \pi \leq \pi
$$

From the calculations shown in Fig. 5.1b the frequency of the considered modes is seen to 
be a monotonously increasing or decreasing function of the wave number. This renders the resonances denumerable. By using eq. (5.5), a normalized wave number can be associated to each longitudinal resonance frequency, from which the dispersion of that transverse mode is obtained.

Figure 5.4 shows the measured transmission and reflection power spectrum of the unperturbed photonic crystal slab resonator in the range from $8 \mathrm{GHz}$ to $13.5 \mathrm{GHz}$ on a logarithmic power scale. For most of the measured frequencies the reflection is close to $0 \mathrm{~dB}$ which is equal to the input power of $1 \mathrm{~mW}$. Due to the resonator an electromagnetic field can only build up at frequencies where longitudinal resonances of a transverse photonic crystal mode exist. In both spectra we clearly observe the resonances belonging to the longitudinal modes and the frequency of reflection and transmission resonances agree very well with each other.

By comparing the transmission levels of the various detected resonances we identify which resonances belong to the same transverse mode. Four frequency ranges can be distinguished in Fig. 5.4. From 8.0 GHz to $9.8 \mathrm{GHz}$ sharp resonances appear with an averaged normalized transmission of about $-15 \mathrm{~dB}$. From $9.9 \mathrm{GHz}$ to $10.7 \mathrm{GHz}$ the transmission is very low $(<-40 \mathrm{~dB})$ and only one weak and broad resonance is observed. However, this is not a stop band of the slab, but a mode that cannot be effectively excited with a centered Hertzian dipole antenna due to its mode symmetry (Fig. 5.2). From $10.8 \mathrm{GHz}$ to $11.8 \mathrm{GHz}$ another set of resonances appears. This set of resonances is easily distinguished from the following set between $11.8 \mathrm{GHz}$ to $13.3 \mathrm{GHz}$ whose average transmission level $(-10 \mathrm{~dB})$ is much higher.

The three different sets of resonances are identified as three different transverse modes. To retrieve the dispersion of each transverse mode we assign a wave number to the longitudinal resonances of each transverse mode. As an example of this process we will concentrate on the frequency range from $8.0 \mathrm{GHz}$ to $9.8 \mathrm{GHz}$. Fig. 5.5a zooms into the reflection and transmission spectrum for this frequency range. For our photonic crystal slab of 15 unit cells, theory predicts to observe 15 longitudinal resonances belonging to mode 1. Indeed, on inspection of the transmission and reflection spectra, 15 resonances are observed. The first resonance is only clearly visible in the reflection spectrum. Furthermore, the resonances above the tenth one are only visible in the transmission spectrum due to a lower signal-to-noise ratio in the reflected signal.

We have used eq. (5.5) to assign wave numbers to each measured resonance frequency of mode 1, as plotted in Fig. 5.5b. The agreement with the theoretical dispersion (solid lines) is excellent. To assign wave numbers to higher order modes we take into account, as explained above, that mode 2 is not effectively excited with the centered Hertzian dipole 
(a)

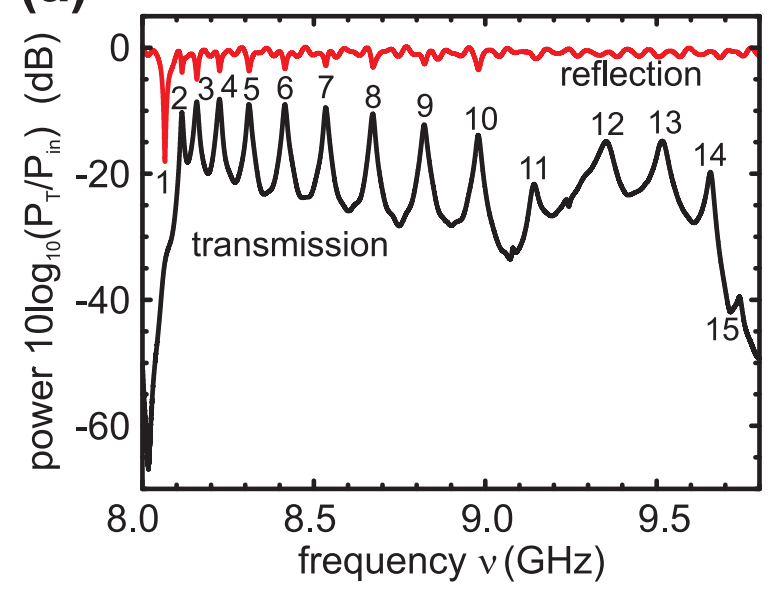

(b)

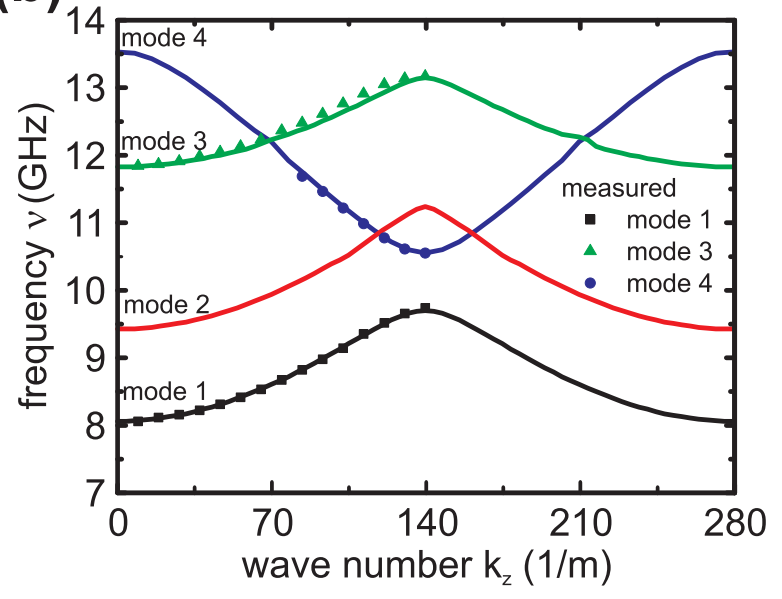

Figure 5.5: (a) Zoom into the transmission and reflection spectrum of the photonic crystal slab depicted in Fig. 5.4. The spectra clearly show the different longitudinal modes for transverse mode 1 . The labeling depicts the number of anti-nodes, $m$, along the propagation direction. (b) Measured band structure of the photonic crystal slab for the mode 1 to mode 4 (symbols) compared to the calculated values from Fig. 5.1b

antenna. Furthermore, the resonances of mode 4 can only be partly observed. Mode 4 overlaps in frequency with mode 3 , but mode 3 couples better to the antenna as can be seen from the higher transmission level of mode 3 . Hence, only mode 3 is detected where both modes overlap.

With these considerations we can assign normalized wave numbers to mode 3 and mode 4, as well. For these modes the agreement with the theoretical dispersion (solid lines) is also excellent. In conclusion, the excellent agreement for mode 1 to mode 4 indicates an appropriate description of the fabricated slab with FDTD calculations.

\subsection{Electric field measurements}

We measure the longitudinal electric field inside the photonic crystal slab by measuring the frequency shift of an individual longitudinal resonance due to a spherical metal scatterer inside the crystal. By scanning the position of the scatterer we can map the electric field distribution. Note that the measured frequency shift $\Delta \nu$ is referenced to the resonance frequency of the resonator loaded by the nylon string alone. The effect of the nylon string is small and results in a shift of only $250 \mathrm{kHz}$.

Figures 5.6a and 5.7a show two examples of the measured frequency shift $\Delta \nu$ for mode 1 due to the scatterer. The frequencies of the two longitudinal resonances are $8.16 \mathrm{GHz}$ 


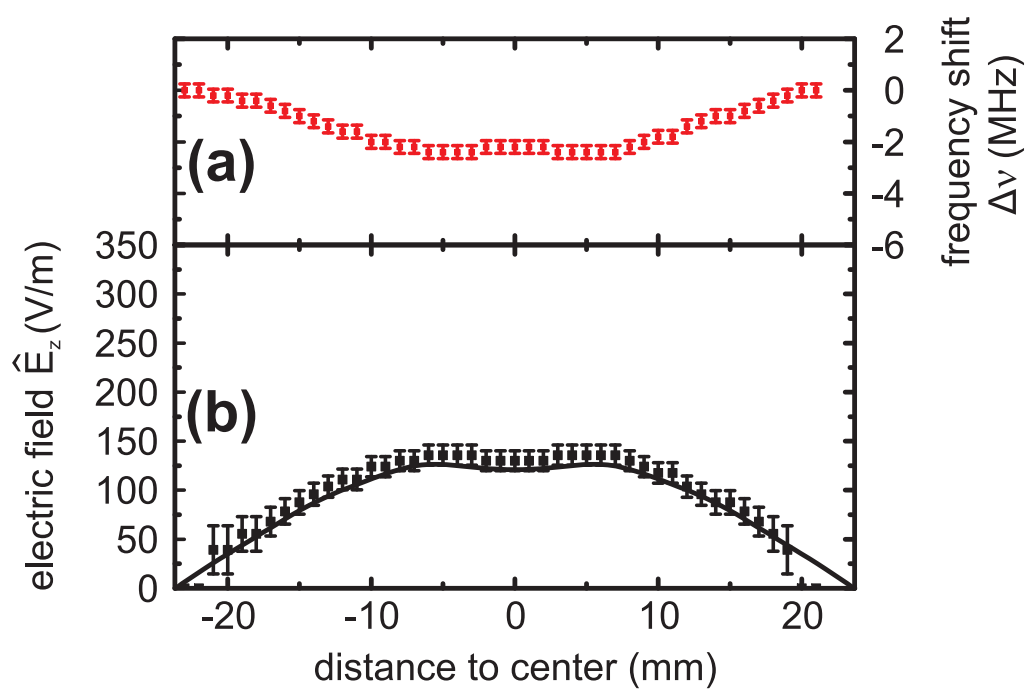

Figure 5.6: (a) Measured frequency shift $\Delta \nu$ induced by placing the spherical scatterer at a certain distance to the center. Shown is the frequency shift $\Delta \nu$ for mode 1 at a longitudinal resonance with $\nu=8.16 \mathrm{GHz}$ and $k_{z, 0} a_{z, \text { eff }}=\frac{1}{15} \pi$. (b) Resulting electric field component $\hat{E}_{z}$ from the measured frequency shift shown in (a) versus position. The measured electric field component $\hat{E}_{z}$ (symbols) is compared to the calculated electric field (lines).

and $8.42 \mathrm{GHz}$, respectively. The uncertainty of the frequency shift is $250 \mathrm{kHz}$ due to the frequency resolution of the network analyzer. In both measurements the frequency shift always remains smaller than or equal to zero, as expected from eq. (5.2) for a mode with a dominating $\hat{E}_{z}$ field component. Towards the edge of the waveguide the frequency shift $\Delta \nu$ approaches zero. As can be seen from Fig. 5.2, the electric field $\hat{E}_{z}$ at these locations is nearly zero. Thus, also the frequency shift by the bead is minimized. In both examples, the strongest frequency shift of about $4.75 \mathrm{MHz}$ and $2.25 \mathrm{MHz}$, respectively, is reached at about $7.0 \mathrm{~mm}$ from the center. In between two rows of rods, the strongest $\hat{E}_{z}$ field component along the $\mathrm{x}$-direction is generally not located at the center of the waveguide but located slightly off the center, see Fig. 5.2.

To calculate the longitudinal electric field $\hat{E}_{z}$ from the measured frequency shift we apply eq. (5.3). However, this requires the determination of the total energy stored inside the cavity $U$. To retrieve $U$ we use the definition of the quality factor [169]:

$$
Q=2 \pi \nu_{0} \frac{U}{P_{\text {diss }}}
$$

Exactly at resonance the dissipated power per cycle $P_{\text {diss }}$ is equal to the input power $P_{\text {in }}$ from the network analyzer. To retrieve the experimental Q-values we determine the full width half maximum of each transmission resonance $\nu_{\text {FWHM }}$ shown in Fig. 5.5a and use 


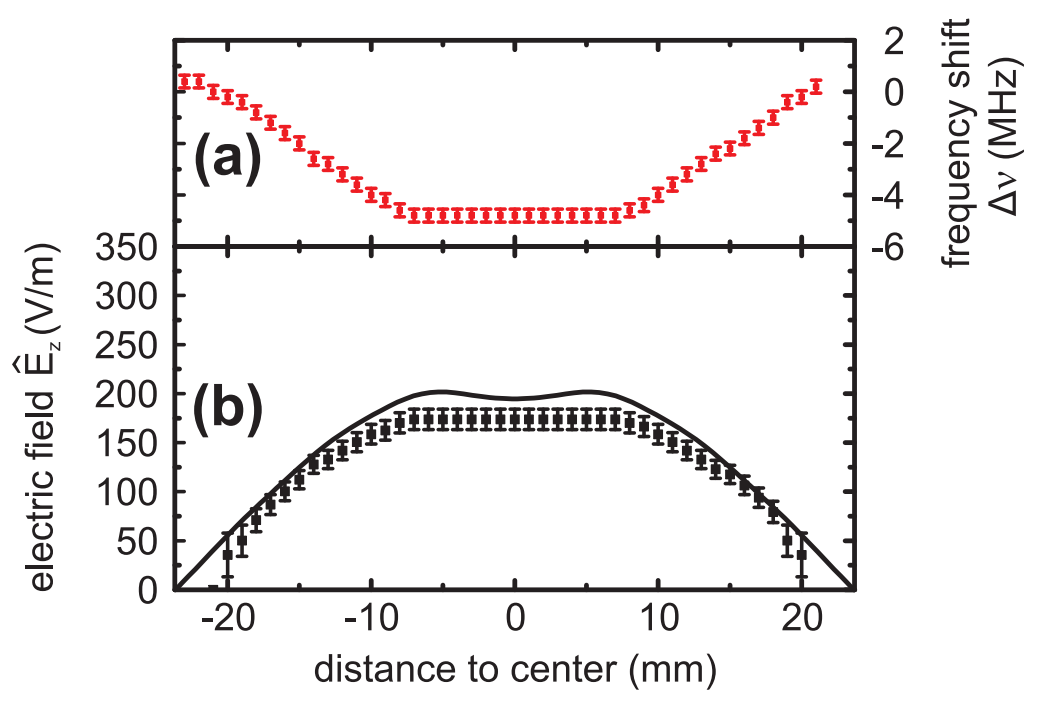

Figure 5.7: (a) Measured frequency shift $\Delta \nu$ induced by placing the spherical scatterer at a certain distance to the center. Shown is the frequency shift $\Delta \nu$ for mode 1 at a longitudinal resonance with $\nu=8.42 \mathrm{GHz}$ and $k_{z, 0} a_{z, \text { eff }}=\frac{6}{15} \pi$ (b) Resulting electric field component $\hat{E}_{z}$ from the measured frequency shift shown in (a) versus position. The measured electric field component $\hat{E}_{z}$ (symbols) is compared to the calculated electric field (lines).

$Q=\nu_{0} / \nu_{\mathrm{FWHM}}$.

Figures $5.6 \mathrm{~b}$ and $5.7 \mathrm{~b}$ shows the resulting electric field strength $\hat{E}_{z}$ (square dots) determined for the two longitudinal resonances of mode 1 at a frequency of $8.16 \mathrm{GHz}$ and $8.42 \mathrm{GHz}$, respectively. As a comparison, the figure also displays the $\hat{E}_{z}$ field strength from the FDTD calculations presented in section 2 (black line). Uncertainties for the measured field values are determined by using the Gaussian error propagation law. We assume that the dominating errors are the uncertainty in input power of about $10 \%$ and the frequency resolution of $250 \mathrm{kHz}$. All other uncertainties are much smaller and do not significantly contribute to the error bars of the measurement.

Figure 5.6 shows the results for a longitudinal resonance with a frequency of $8.16 \mathrm{GHz}$. The overall shape of the electric field shows an excellent agreement to the calculated field strength. Furthermore, the absolute values agree within the range of the measurement accuracy, although no adjustable parameter is used in the calculations. Figure 5.7 shows an example at a higher frequency of $8.42 \mathrm{GHz}$. The agreement between FDTD calculations and experiment is, again, very good. This holds for both the shape of the profile and the absolute values. Minor deviations are visible at the waveguide center. The small difference between the electric field maximum and the central electric field value at the center is calculated to be about $7 \mathrm{~V} / \mathrm{mm}$. This is not resolved in the measurements. In addition, 
the measured absolute field value is slightly lower. We might tentatively attribute both effects to the fact that influences due to non-periodic variations in the photonic crystal become more important at a higher frequency (shorter wavelength). Nevertheless, in both examples a good agreement between experiment and theory is found.

The two discussed examples for mapping the electric field component $\hat{E}_{z}$ effectively illustrate the capability of the method to map the absolute value of a specific electric field component inside a photonic crystal.

\subsection{Conlcusion and outlook}

We have demonstrated for the first time a method for mapping the absolute strength of an electromagnetic field component inside a photonic crystal. The method relies on measuring the change in resonance frequency when the photonic crystal is placed inside a resonator and the field inside the photonic crystal is perturbed by a sub-wavelength scatterer. In our experiments spherical scatterer is applied to measure the dominating longitudinal electric field $\hat{E}_{z}$ in a specific photonic crystal slab. We observe good agreement between measured and calculated electric field strength $\hat{E}_{z}$ without using any adjustable parameters in the calculations.

Note that if all six field components are of comparable strength, such as in an arbitrary photonic crystal or at specific locations, this measurement method can still measure each individual field component. For example, the strength of the electric field components can be individually measured by using a metallic needle instead of a metallic bead. The metallic needle short circuits and thus probes the electric field along the orientation of the needle while leaving all other field components unaffected. For a thin metallic needle with a thickness $d$ and a length $l$ aligned along the $z$-direction one obtains [240, 241]:

$$
\begin{array}{r}
\hat{E}_{z}(\mathbf{r})=\left(-12 \frac{\Delta \nu(\mathbf{r})}{\nu_{0}} \frac{1}{\pi l^{3}} \frac{\ln \left(1+\sqrt{1-(d / l)^{2}}\right)}{\left(1-(d / l)^{2}\right)^{3 / 2}} \ln \left(1-\sqrt{1-(d / l)^{2}}\right)\right. \\
\left.-\frac{2 \sqrt{1-(d / l)^{2}}}{\left(1-(d / l)^{2}\right)^{3 / 2}}\right)^{1 / 2}
\end{array}
$$

In the near future, we plan to apply this method to map and compare the field distribution of various photonic crystals for use in our photonic crystal lasers. Further we envision that this method could be even applied in the near infrared or optical domain to characterize general photonic crystals by scaling down the setup. Instead of a bead on a string we suggest mounting a metallic or dielectric scatterer on one end of a stiff 
carbon nanotube whose other end is mounted on the tip of an atomic force microscope. The scatterer is then moved by moving the atomic force microscope tip. Mounting a carbon nanotube on an atomic force microscope has been demonstrated [244]. Further, atomic force microscopy allows a sufficiently high spatial resolution, as demonstrated by recent measurements where an emitter directly mounted to an atomic force microscope has been used to map the emitter lifetime around a single nanorod [245]. Combining these techniques, it should be possible to measure the shift in resonance frequency in the transmission spectrum to determine the absolute field strength inside accessible air voids of an photonic crystal at optical frequencies. 



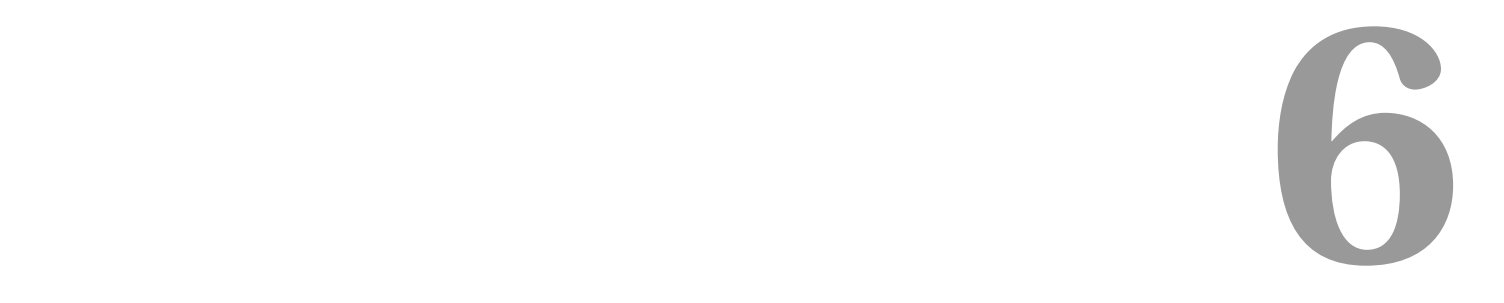

Design of a photonic free-electron

laser

\subsection{Introduction}

In chapter 3 and chapter 4 we have given a thorough numerical investigation of a pFEL, showing that such a laser is promising for the microwave regime and, possibly, also beyond microwave frequencies towards the THz range. So far, the only experimental study of such a laser does not seem to have employed the significant slowing of phase and group velocity possible in a photonic crystal. Instead pumping has been performed with high electron energies of $E_{e l}>200 \mathrm{keV}$, which is more than an order of magnitude higher than what we propose in the numerical calculations. Further, those experiments have been using huge beam currents beyond $I>1000$ A [246, 247], a choice that excludes continuous steady-state laser operation with a well defined output quality of the radiation. The described megawatt pumping could only be applied in a pulsed manner, i.e., with a strongly time dependent beam energy and current, causing that the laser never reaches steady-state in these experiments [246, 247]. This makes a comparison between theory and experiment effectively impossible. Also, because the beam current and voltage varies continuously, very strong magnetic fields, in excess of $1.5 \mathrm{~T}$, are required for beam guiding 
[246, 247]. However, such high magnetic field strength also enables Gyrotron emission which is conceptually different from pFELs [170]. Having two different gain mechanism simultaneously present in a laser makes it difficult to interpret the experimental results and compare these with theory. In conclusion, to study the physics of a pFEL it would be desirable to design a laser in which the experimental parameters can be controlled and only a single gain mechanism is present, such that the laser output can be well analyzed. This would enable an accurate comparison between theory and experiment.

Properly designed pFELs allow for pumping with low-energy and low-current beams which allows to operate the laser in steady-state and to characterize it thoroughly. This allows the use of different technologies than those discussed above. Thereby, a much better control of the electron beam is possible. For example, in the traveling-wave tube, one of the most widely used low-energy, slow-wave FELs, it became even possible to measure the phase space distribution of the electrons after interaction [164]. While the know-how from traveling-wave tubes provides a solid technological foundation for pFEL experiments traveling-wave tubes typically only employ single electron beams. Thus, multi-beam electron sources are not easily available and would have to be individually designed [248-250]. Further, as we have seen in chapter 4 , the operation of multi-beam pFELs is more difficult to understand and, thus, to predict due to the complexity introduced by mode competition.

In this chapter we outline the design of a microwave pFEL pumped by a single, lowenergy, electron beam $\left(E_{e l}<15 \mathrm{keV}\right)$ and that, we believe, can be realized and brought to operation in the near future. The design of the pFEL is based on the calculations presented in chapter 3. The first section describes the overall setup, while the remaining sections describe the development, modeling and characterization of the individual components of the setup. We start with describing the electron beam generation by the electron source. Next, we describe the beam transport to be achieved via a solenoid magnet. Finally, we present the design and fabrication of the microwave components which encloses the pFEL structure, i.e., the resonator including the photonic crystal slab and the vacuum window.

\subsection{Setup overview}

The goal of the experiments is to realize, for the first time, a pFEL based on low-energy electrons suitable for a complete investigation of the underlying physics. A systematical study of this kind has so far only been done theoretically via numerical modeling which has been described in this thesis. The operation of this pFEL has to be controllable and measurable to such an extent that an accurate and comprehensive comparison between 
experiments and modeling becomes possible. A precondition for this is a carefully designed laser. The most critical components of the laser are a well-controllable electron beam and a suitable photonic crystal, as the interplay between these provide the amplification. In chapter 3, which describes the modeling, the beam current has been chosen to be $I_{0}=1 \mathrm{~A}$, and beam energies have been selected to range from $E_{e l}=10 \mathrm{keV}$ to $15 \mathrm{keV}$. Assuming a strong magnetic field of $0.5 \mathrm{~T}$, the beam would be guided throughout the structure in a beam radius of $r_{b}=1 \mathrm{~mm}$. The modeling then predicts the generation of electromagnetic waves with a frequency of about $16 \mathrm{GHz}$. The dimensions of this metallic photonic crystal slab are in the mm-range and given in detail in Fig. 3.2. The complete crystal has been considered to be enclosed by a resonator. All metallic parts have been assumed to be perfectly conducting throughout the modeling. Finally, the numerical results have predicted the power, frequency and modal content that would be measurable behind the photonic crystal slab in a rectangular waveguide under vacuum.

In practice none of the conditions can be exactly realized in an experiment. However, with a proper design it should be possible to approximate all of the desired parameters sufficiently well. A first issue is the launching of the electron beam. In the modeling, a certain area of the perfect metal mirror emits the electron beam for numerical convenience, while the beam radius remains approximately constant throughout the rest of the crystal volume via the application of a strong, homogeneous magnetic field. Real electron sources combined with a proper guiding technique can provide a nearly constant electron beam radius throughout the volume of the crystal. However, such sources can not be integrated into the photonic crystal. Thus, for the real design we decided to inject the electrons via an injection hole in the upstream mirror (plane A) of the resonator which alters the resonator design somewhat. Further, real electron sources apply high-voltages and require a vacuum. In addition, providing high magnetic fields for guiding the beam with a constant beam radius is difficult and an alternative method using smaller magnetic fields for the guiding is desirable. A second issue is the choice of materials for the photonic crystal slab and the resonator. Specifically, the assumption that all metallic parts are perfectly conducting can only be met approximately. While metals have, indeed, a high conductivity, residual losses are always present [165]. These additional material losses increase the pump current threshold. In order to make sure that the threshold current can be reached, it is desirable to minimize these losses by selecting appropriate materials. In addition, the selected type of electron source should still be easily capable to provide more than the correspondingly increased threshold current. A third issue is how to perform measurements of the input and output quantities. Measuring inside a vacuum vessel is generally quite complex, because one can not easily exchange or freely 


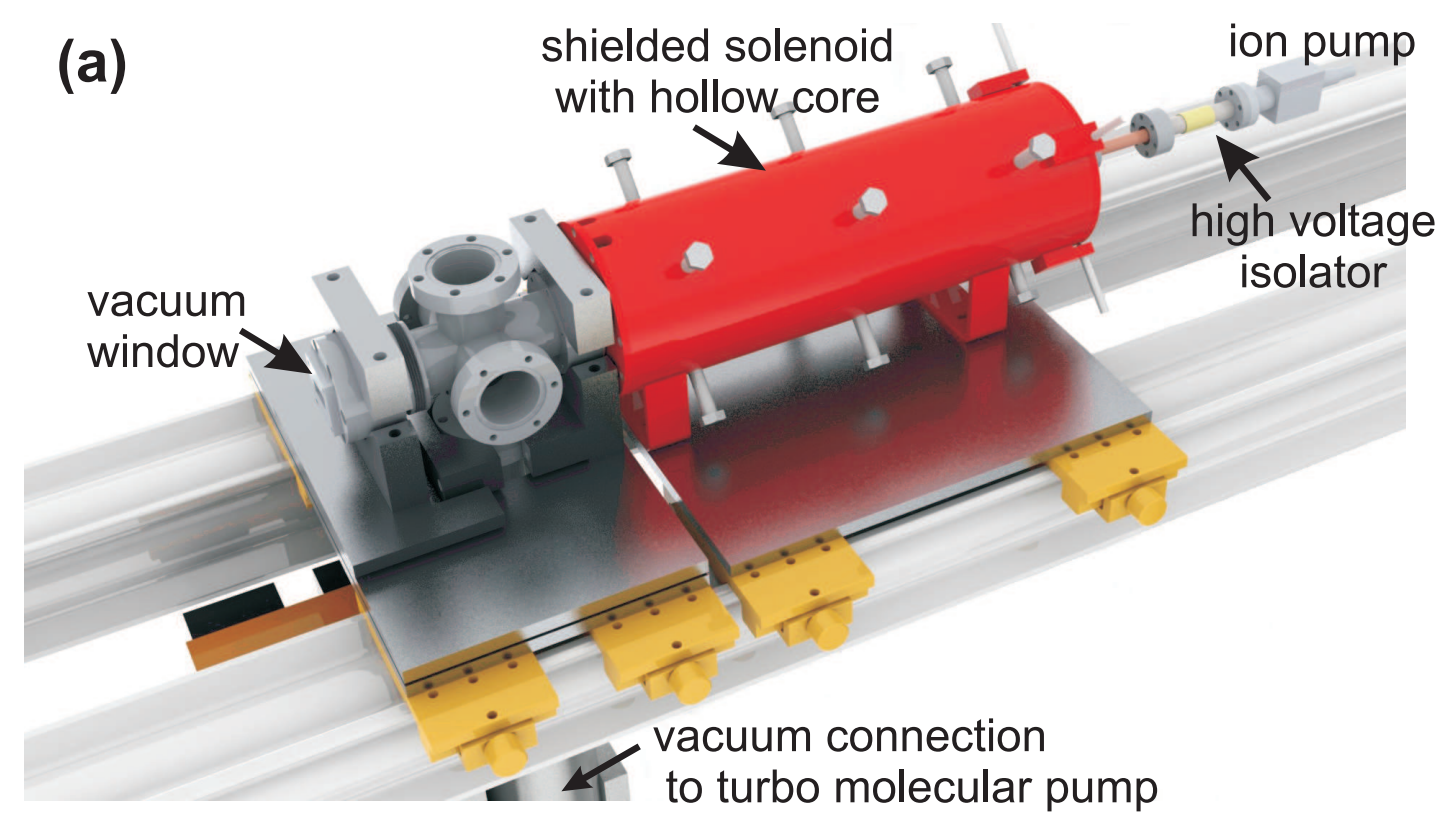

(b)

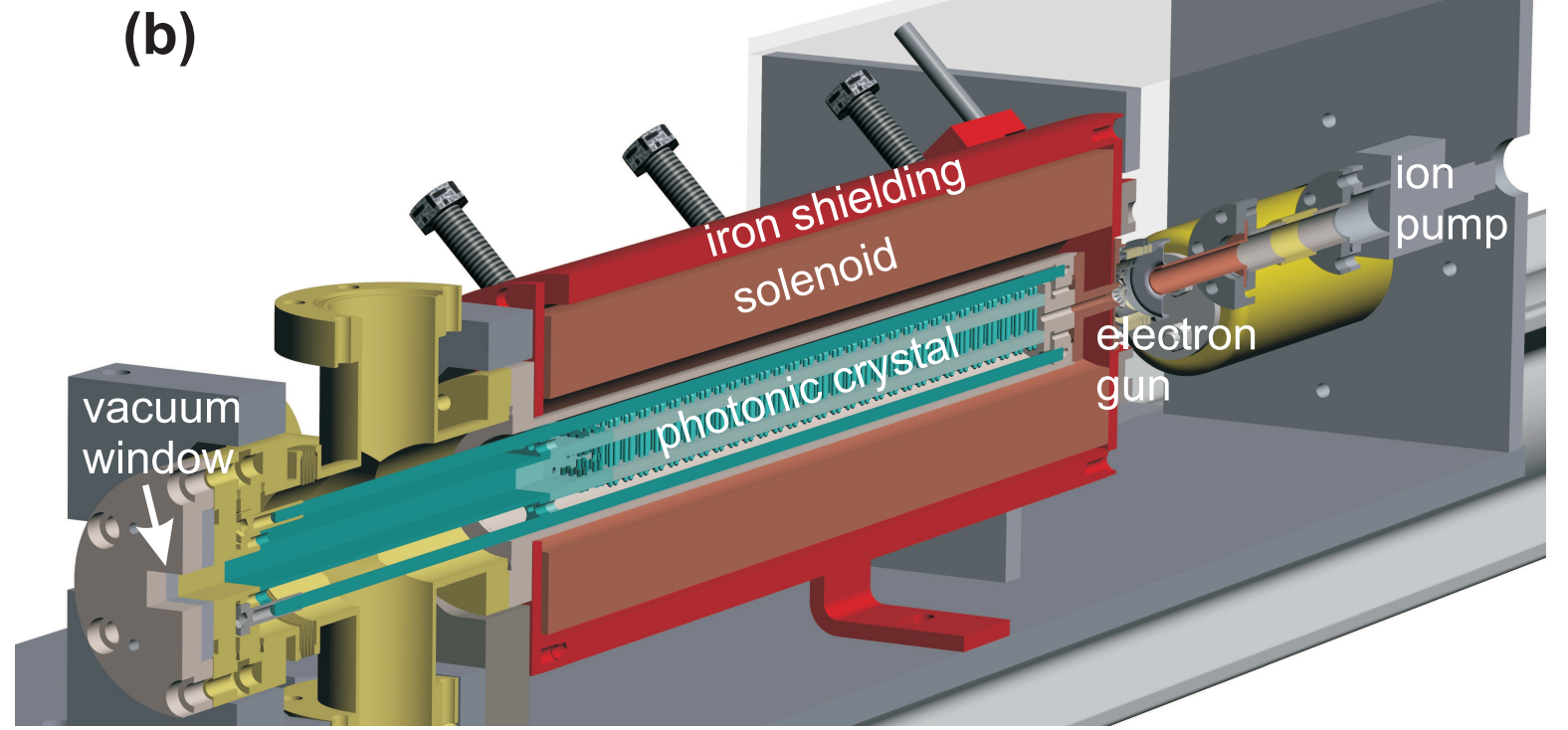

Figure 6.1: (a) A rendered three-dimensional view of the pFEL construction model. Electrons are injected by an electron gun into a solenoid magnet with a hollow core (in red). Note that the electron gun itself is hidden in this view, only the high-voltage isolator and the ion pump are visible, which are connected to the backside of the electron gun. For establishing an ultra-high vacuum inside the vessel, next to the ion pump, a turbo molecular pump is connected to the setup. (b) Cross section of the pFEL design. In this view the electron gun and the photonic crystal slab inside the magnet are visible as well. After radiation is emitted inside the photonic crystal slab it propagates down an empty rectangular waveguide towards a vacuum window. 
position measurement equipment. Thus, measuring the spatial and spectral content of the generated radiation should preferably be done outside the vacuum. This is possible but requires to include a suitable vacuum window in the design to couple the radiation out into a waveguide at atmospheric pressure. In summary, the design work requires to combine a wealth of vacuum requirements, high-voltage requirements, microwave engineering, and electron beam transport in the construction.

Over the course of this thesis a design has been developed which is optimized to match all these conditions. An overview of this design is shown in Fig. 6.1. While Fig. 6.1a shows a three-dimensional, rendered image of the complete design, Fig. 6.1b gives a crosssectional view. The electrons are emitted by a commercial, thermionic electron gun which provides a nominal electron current of about $2 \mathrm{~A}$ at a low energy of about $14.2 \mathrm{keV}$. The beam guiding with a magnetic field through the photonic crystal slab will be achieved via the so-called Brillouin flow technique, which minimizes the required magnetic field strength [89]. For this guiding technique a shielded solenoid is required - the shielding is indicated in red in Fig. 6.1a. Behind the photonic crystal slab, the solenoid magnet ends and due to the shielding a nearly field-free region is found a few centimeters away from either side of the magnet. Consequently, the electron beam diverges and the electrons hit the inner walls of the output waveguide. For matching the size and impedance of the pFEL's output waveguide to a standard waveguide, the pFEL's output waveguide is internally tapered to connect to a standard waveguide size (WR90) [165]. At the end of this standard waveguide, a thin window made from Kapton foil transmits the generated output into a waveguide at atmospheric pressure and characterization of the expected microwave output can be performed outside the vacuum vessel.

In the following sections we describe all integral parts of this design in more detail, starting with the electron gun. Thereafter, we describe the electron beam transport and, finally, we describe the realization of the photonic crystal slab and the output window.

\subsection{Electron generation}

While many different types of cathodes are available, standard electron guns used for slow-wave FELs employ thermionic dispenser cathodes, because these are well-developed and can sustain a high current [63]. For the pFEL we have also selected such an electron gun type, which is normally used in certain traveling-wave tubes [251]. While the high beam current of dispenser guns is highly desirable for generating stimulated emission, this comes at the expense of high demands on the vacuum system [235-238]. To prevent destruction of the dispenser cathode by so-called poisoning, the vacuum pressure should 


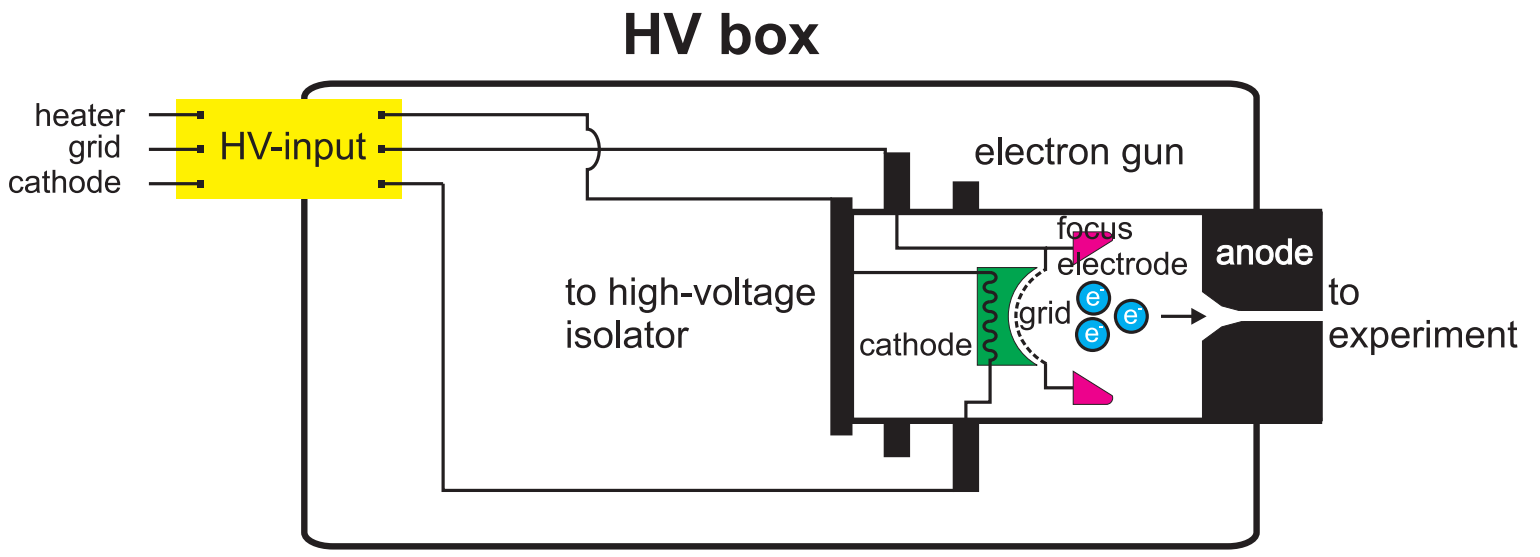

Figure 6.2: Cross-section of the electron gun and the electrical connections for operating it.

preferably remain below $1 \cdot 10^{-7}$ mbar. Further, after first operation one should limit the number of times the cathode is exposed to air. According to the manufacturer, our specific gun should not be exposed to air more than a few times [239].

The cathode emits a high current of free electrons. In order to apply these electrons for driving the pFEL, a concise, well-defined beam must be formed and accelerated to an energy of about $15 \mathrm{keV}$. As is typical for thermionic electron guns, the beam in our gun is formed by an electrostatic focusing electrode and accelerated by a high-voltage gradient $U_{0}$ between anode and cathode, see Fig. 6.2. The accelerated electron beam leaves the gun through a hole in the anode. The specifications of our electron gun are the following. The beam energy ranges from $7 \mathrm{keV}$ to $15 \mathrm{keV}$, which can be easily adjusted by changing the acceleration voltage between anode and cathode. The nominal beam energy of the gun is about $14.2 \mathrm{keV}$, where the gun emits a high beam current of $2 \mathrm{~A}$. These parameters define the so-called gun perveance,

$$
P=I_{0}\left(\frac{e}{E_{e l}}\right)^{3 / 2}
$$

The perveance is, in a good approximation, only dependent on the gun geometry [89, 252, 253]. Thus, the beam current at other beam energies can be calculated by assuming that the gun perveance of $P=1.16 \mu \mathrm{AV}^{-3 / 2}$ remains constant. Finally, the beam radius at the focus point of the gun is $r_{b}=1 \mathrm{~mm}$. From these specifications the nominal beam power amounts to about $30 \mathrm{~kW}$. Such high power is difficult to handle continuously, as the power supply load is high and the thermal design, especially of the beam dump, becomes very challenging at such excessive average powers. Therefore, we plan to operate the gun in a 
pulsed mode, with pulse durations of about $10 \mu \mathrm{s}$ and a repetition rate of $10 \mathrm{~Hz}$, meaning that the duty cycle is $0.1 \%$. This allows using the waveguide wall as beam dump as it lowers the average beam power to about $3 \mathrm{~W}$. We note that pulse durations of a few microseconds are certainly long enough to allow a study of all the dynamical aspects of the laser, because steady-state operation is already expected after a few hundred nanoseconds (see chapter 3). For pulsing, the electron beam the gun is equipped with a grid electrode in front of the cathode. As the grid is placed in close proximity to the cathode, a potential change of only a few hundred volts is sufficient to start or to suppress electron emission. In addition, to avoid excessive heating of the grid during the electron pulse, the cathode is equipped with a shadow mask which restricts the electron emission to cathode areas that are directly below the open areas of the grid.

To operate the electron gun, several different voltages have to be supplied to the electron gun as shown in Fig. 6.2. A high-voltage potential is used for acceleration, while low voltages operate the heater $(6.3 \mathrm{~V})$, the grid (about $\pm 300 \mathrm{~V}$ ) and the focus electrode (connected to the grid). For safety reasons, it is convenient and common practice to ground the anode. The cathode voltage is then biased to a negative high-voltage potential. In this layout, none of the high-voltage electrodes is accessible to the user - and could endanger him or her. However, the lower voltages then have to float on a high potential and a floating power supply must be designed.

Figure 6.3 shows the scheme of our home-built floating high-voltage power supply. To make those power supplies which float on a high-voltage physically inaccessible to the user, the whole structure is placed in a grounded metal tank. The tank containing the floating supplies has electrical feed-throughs, both for the high-voltage and for the low-voltage input and output signals. As we use a pulsed electron beam we base the power supply on a capacitor charging scheme. The capacitor $(C=1 \mu \mathrm{F})$ is charged with a high-voltage of negative polarity using a commercial power supply, placed outside the tank. The power supply delivers a maximum current of $30 \mathrm{~mA}$. When the capacitor is fully charged, an electron beam current of $2 \mathrm{~A}$ during a $10 \mu$ s pulse only changes the potential between the two capacitor terminals by $20 \mathrm{~V}(0.1 \%)$. Thus, a nearly constant voltage remains present between anode and cathode during the capacitor discharge pulse. The small charge drawn from the capicator during a single pulse can be easily replaced between two consecutive electron pulses. To monitor the voltage between the capacitor terminals a voltage divider is connected to one of the terminals. The divider consists of a $40 \mathrm{M} \Omega$ connected in series to a $1 \mathrm{k} \Omega$ resistor. By measuring the low voltage present over the $1 \mathrm{k} \Omega$ resistor we can compute the high-voltage present between the capacitor terminals. Heater and grid supply float on this high-voltage potential. To fine-tune the 
operation of the electron gun, the heater and grid power supply must be adjustable during operation. To adjust both supplies while floating on high voltage they are controlled via a micro-controller, which also floats on the high-voltage potential. The micro-controller is remote-controlled from a PC via three optical fibers, which provide a communication link to the PC and at the same time provide the high-voltage isolation between the input and output. One fiber input is used for triggering the electron beam pulse, while the other two fibers are used for changing the settings of the power supplies. All the floating power supplies inside the tank are powered by a $220 \mathrm{AC}$ voltage which is feed onto the high-voltage via an isolation transformer that provides $100 \mathrm{kV}$ DC isolation between its input and output. Finally, in order to measure the current emitted from the cathode, the supply wire towards the cathode is surrounded by a Rogowski coil. The described power supply has been built and successfully tested.

\subsection{Electron beam transport}

To achieve a high gain in the pFEL, the electron beam has to be guided through the photonic crystal with a high transmission efficiency. In the numerical modeling the total length of the gain section has been selected to be $50 \mathrm{~mm}$, see chapter 3. Further, to prevent a degradation of the gain the velocity spread of the electron beam should be minimized [254]. However, due to their charge, electrons repel each other and therefore the electron beam would quickly diverge without an external force being present. Inside the electron gun, the external force is generated by the focus electrode, which compresses the electron beam to a small diameter in a given distance. This electrostatic focus point lies a few millimeters behind the exit hole of the anode. Beyond this distance the electron beam would diverge again, if no additional external force is applied. The diverging beam would inevitably hit the photonic crystal and no electromagnetic field would be generated. To provide a suitable guiding force that prevents this, a strong magnetic field of $B_{z}=$ $0.5 \mathrm{~T}$ was assumed to be present in the modeling. However, the required magnetic field strength of $B_{z}=0.5 \mathrm{~T}$ is impractical to generate. In commercial applications of high current electron guns an alternating magnetic field from permanent magnets is often applied to guide the electrons [253]. Indeed, such technique has also been developed for the selected electron gun for operation in a traveling-wave tube. While guiding with permanent magnets is desirable for commercial applications due to its low mass and volume, it only works for a specific, fixed acceleration voltage which would prevent any systematic investigation of frequency tuning.

To minimize the required magnetic field strength, it is well-known that so-called Bril- 


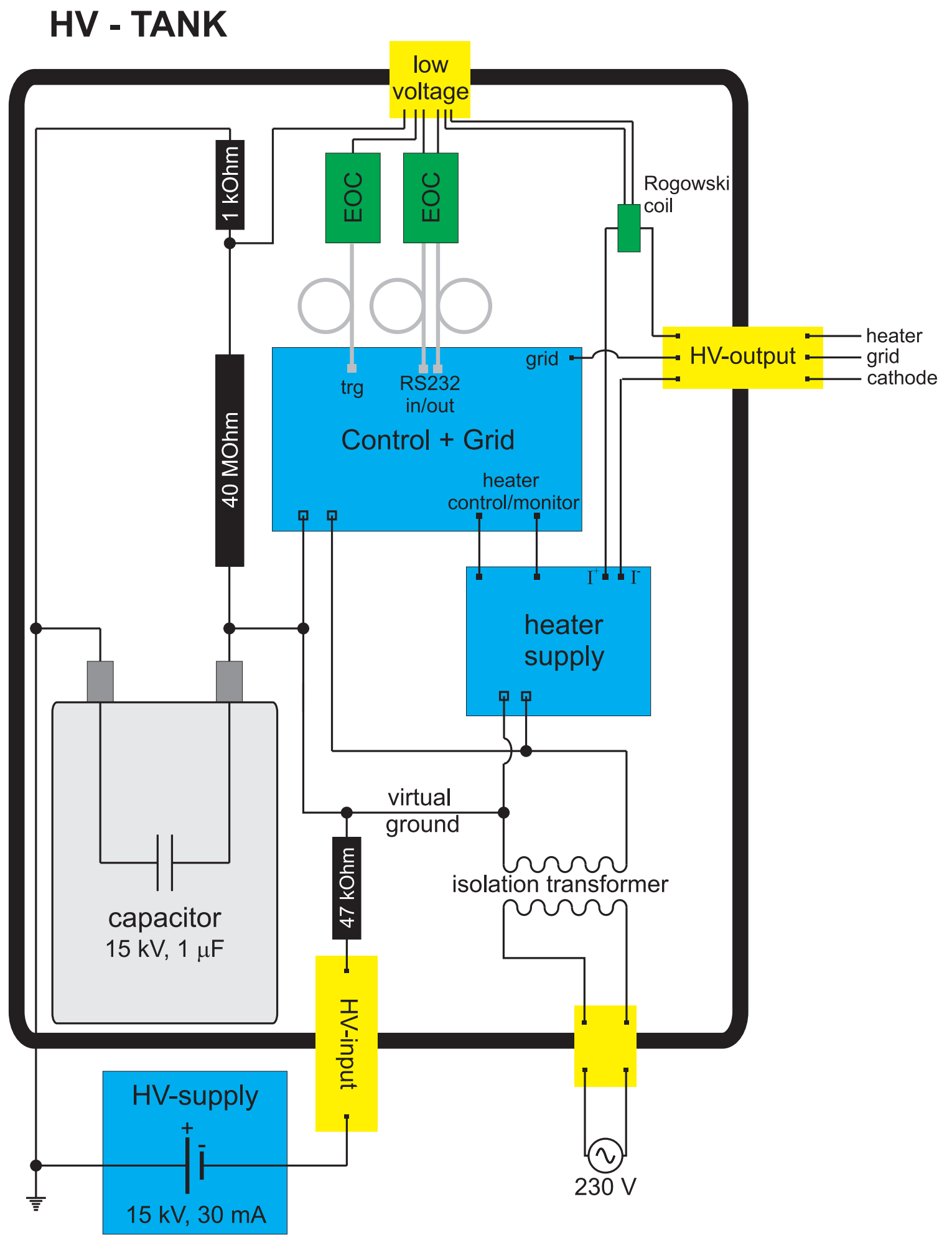

Figure 6.3: Scheme of the electrical circuit for the floating high-voltage power supply that is used to provide the required voltages for the electron gun. 
louin guiding based on an adjustable magnetic field provides suitable guiding of electron beams $[66,89,252]$. In this guiding technique, the field is assumed to be zero at the cathode region and needs to abruptly increase at the focal point of the electron gun. Beyond the focal point a constant magnetic flux density, $B_{z}$, needs to be abruptly established in the guide the electron beam. The required value of the flux density in the guiding region is given by [89]:

$$
B_{z}=0.83 \cdot 10^{-3}\left[\frac{\mathrm{TmV}^{1 / 4}}{\mathrm{~A}^{1 / 2}}\right] \frac{I_{0}^{1 / 2}}{U_{0}^{1 / 4} r_{b}} .
$$

where $r_{b}$ is the beam radius at the focal point of the gun. The evaluation of this expression for the selected gun with $r_{b} \approx 1 \mathrm{~mm}$ shows that Brillouin guiding requires a flux density of approximately $B_{z}=108 \mathrm{mT}$ at the nominal electron beam energy. Generating a field with such flux density can be easily done with a hollow core solenoid magnet. However, without further precautions the flux density only reduces very slowly towards zero magnetic field outside the solenoid magnet. Providing the required abrupt increase of the magnetic field at the entrance of the magnet can be achieved by completely enclosing the magnet with an iron shield. As iron is ferromagnetic, it short-cuts the external magnetic field lines such that these field lines do not reach the cathode region. Further, the magnetic field on the inside of the solenoid becomes more homogeneous. While such a shield, when fully closed, provides the ideal magnetic field shape, this is not very useful for a real design. In order to inject the electron beam into the magnetic field at the upstream end a vacuum tube from the gun has to be fitted through the shield. Also, at the downstream side another vacuum tube should fit through the shield to transport the electron beam to the beam dump and the generated radiation to the vacuum window of the vessel. Both constraints lead to magnetic field leakage into the surrounding region, which reduces the homogeneity of the field inside the magnet. In addition, having a non-zero magnetic field at the cathode location can potentially deteriorate the guiding of the electron beam. Next, it must be ensured that for any shield design the focal point from the electron gun can be brought to an overlap with the sharp increase of the magnetic field. Finally, in practice it is often found that the magnetic field for Brillouin flow should be about two times stronger than is predicted by eq. (6.2), [255]. This is because Brillouin guiding assumes a laminar flow of the electrons, which is not the case in a realistic electron gun. First, due to the elevated temperatures at the cathode, emitted electrons always possess a certain transverse velocity. Second, the grid introduces further transverse velocities, due to a slight potential variation.

In order to find a shielding that takes non-laminar electron flow and the geometrical 


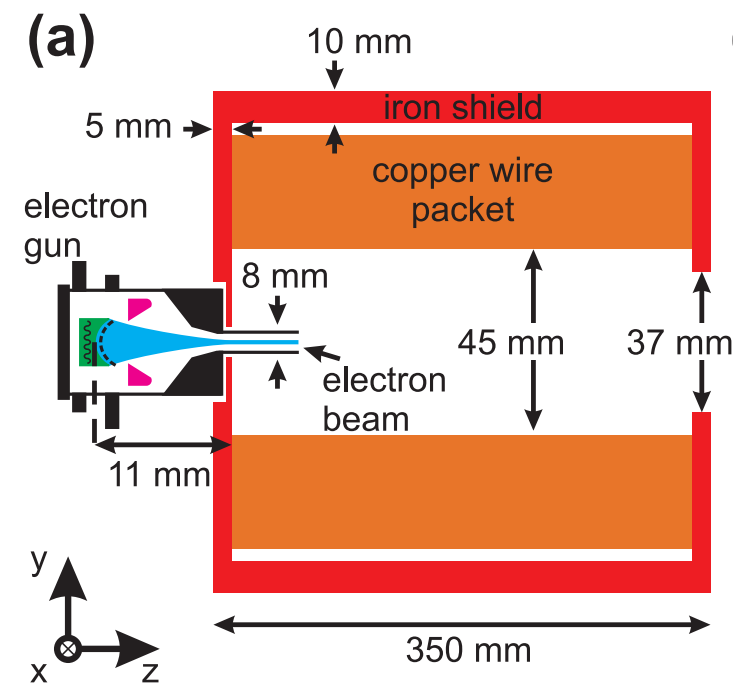

(b)

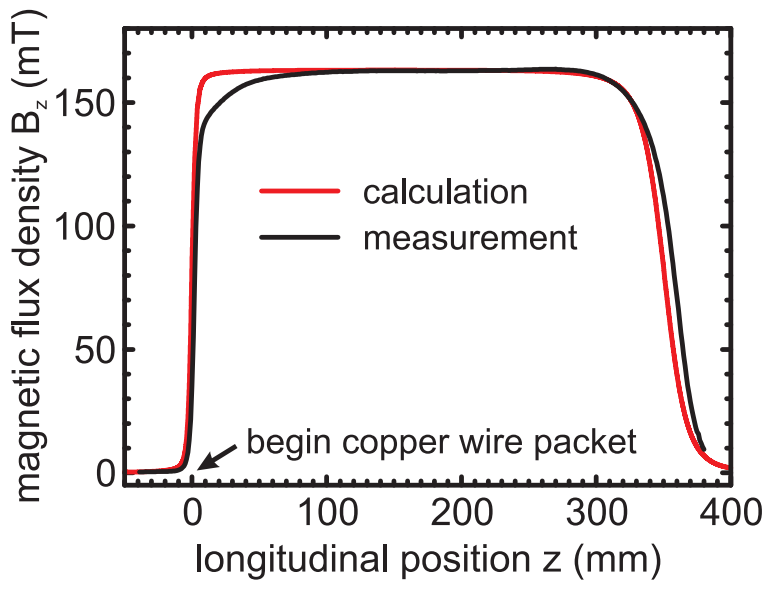

Figure 6.4: (a) Cross-section through the design of the solenoid magnet and the iron shield (b) Calculated and measured magnetic flux density along the axis of the solenoid magnet.

limitations into account, but still enables Brillouin guiding, we take the following three steps. First, we calculate the solenoid field for a certain shield with a three-dimensional magneto-static solver [256]. Second, this field distribution is combined with the threedimensional model of the electron gun as provided by the gun manufacturer [251]. Third and finally, we investigate the electron propagation of this combined model with a threedimensional particle tracer program [256]. Note that this model also includes realistic thermionic cathode emission and the actual grid electrodes and potentials [251]. Based on the calculated electron beam trajectories, we then decide whether sufficient guiding is achieved, i.e., a constant beam diameter is observed over several centimeters. If this is not accomplished we re-design the shield until sufficient guiding is observed.

Figure $6.4 \mathrm{a}$ shows the final design for the shielded solenoid from this procedure. It turns out that the required magnetic flux density for guiding is about $B_{z}=163 \mathrm{mT}$ which is about 1.5 times more than that predicted by eq. (6.2). The solenoid providing this magnetic field has a length of $350 \mathrm{~mm}$ and a hollow core with a diameter of $45 \mathrm{~mm}$. To minimize field leakage, the central hole in the shield neatly fits to the vacuum tube diameter with a $0.5 \mathrm{~mm}$ margin for alignment purposes. Taking into account the housing of the electron gun, the maximum thickness of the upstream shield is $1 \mathrm{~mm}$. However, it was found that such a thin sheet of iron becomes saturated in magnetization by the required magnetic flux density for guiding. The consequence is additional leakage of magnetic field. To prevent this additional field leakage, the outer part of the upstream shield is chosen to be much thicker and its thickness is $5 \mathrm{~mm}$. The starting point of this 
thicker shield is chosen such that the gun housing fits into the created cavity. The lateral area of the solenoid is completely covered with a $10 \mathrm{~mm}$ thick shielding. The downstream shielding has a thickness of $5 \mathrm{~mm}$. Further, for fitting the rectangular output waveguide through the solenoid the downstream shield has a larger central opening with a diameter of about $37 \mathrm{~mm}$.

The calculated variation of the magnetic flux density along the axis of the shielded solenoid is shown in Fig. 6.4b. As expected, some field leaks out through the upstream part of the shield into the gun region. As the distance between cathode and focal point of the gun is about $11 \mathrm{~mm}$ the cathode is placed at $z=-11 \mathrm{~mm}$. Note, we verified that slight changes around this value do not significantly influence guiding. The magnetic field at the cathode position is below $3 \mathrm{mT}$, which is less than two percent of the field inside the solenoid. Inside the magnet the field quickly reaches its value of $B_{z}=163 \mathrm{mT}$ and remains very homogeneous. The magnetic flux density varies by less than $2 \%$ inside a range from $10 \mathrm{~mm}$ to $300 \mathrm{~mm}$ and in this range we should achieve guiding.

The calculated electron beam trajectories associated to this final design from the particle tracer code are shown in Fig. 6.5. The figure shows the electron beam trajectories at the nominal beam energy of $14.2 \mathrm{keV}$ projected onto the $\rho$ - $z$ plane, with $\rho$ being the distance to the longitudinal axis. The curved cathode is located at $z=-11 \mathrm{~mm}$. Close to the cathode, at negative $z$-values, the trajectories are seen to be focused by the focus electrode. When the electrons enter the magnetic field at $z=0$ it can be observed that the electrons beam radius is about $1 \mathrm{~mm}$. While the beam should diverge when an improper magnetic field is used, the electron beam radius $r_{b}$ remains approximately $1 \mathrm{~mm}$ throughout the entire calculated distance as desired. As the magnetic field hardly changes beyond $z=65 \mathrm{~mm}$, we expect that the beam will remain guided, until the magnetic field starts to significantly reduce at the downstream end of the solenoid. With this realistic modeling the realization of such magnetic field configuration should allow a high transmission efficiency of the electron beam throughout the whole photonic crystal. Further, we found a small energy spread of less than $2 \%$ for this configuration. We verified that this small energy spread alters the steady-state output and small-signal gain by less than $2 \%$, via running further PIC calculations of the numerical model shown in Fig. 3.1 - We note that transport without any interception was still achieved if we lowerd the magnetic guiding field by $10 \%$. Thus, even when assuming the well-known effect that bunching detertoriates the guiding, it is reasonable to expect that a variation of less than $2 \%$ of the magentic field strength should still allow guiding. Therefore, the interaction length of the photonic crystal might be increased to up to $290 \mathrm{~mm}$. This is about five times longer than the gain section in the numerical calculations. Recalling that in the 

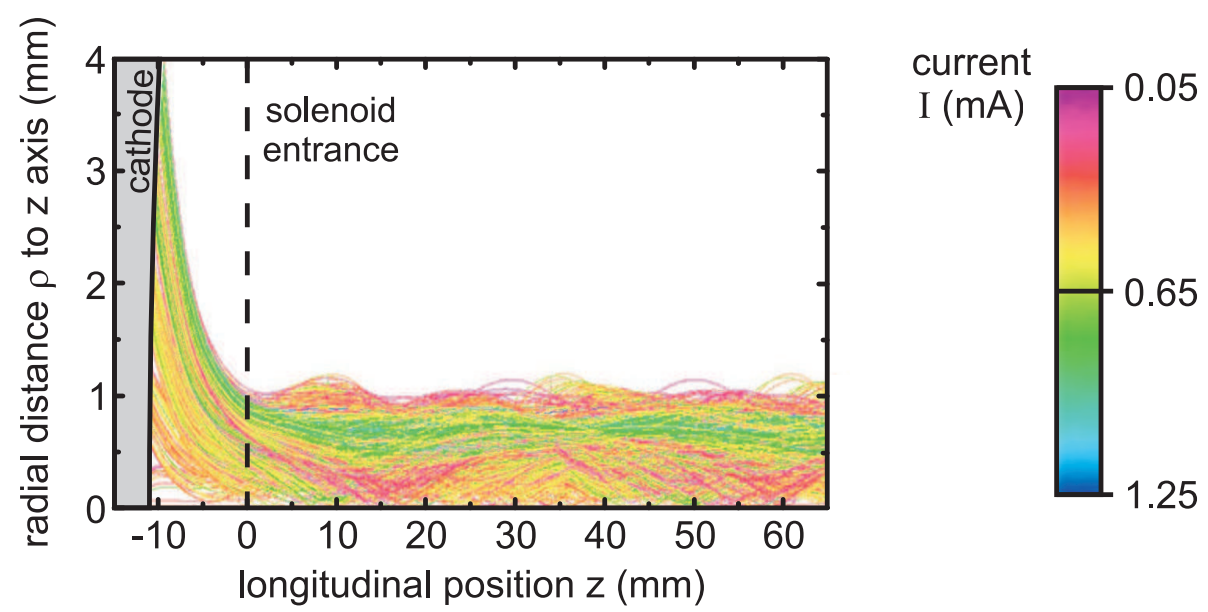

Figure 6.5: Electron beam trajectories from the cathode to the inside of the solenoid magnet, shown in the $\rho-z$ plane. The figure shows the result for the nominal beam energy of $14.2 \mathrm{keV}$, a beam current of $2 \mathrm{~A}$ and a magnetic flux density of $B_{z}=160 \mathrm{mT}$

small-signal regime the amplification per pass grows exponentially with $z$, this should provide ample gain to reach the threshold.

To realize the magnetic configuration we chose an electromagnet. Such a magnet configuration has the advantage that the magnetic field strength is easily adjustable via the current. This is very desirable for performing systematical studies of the tuning of the laser frequency. For experimental simplicity, we chose a normal conductive magnet whose weight we limited to below $25 \mathrm{~kg}$. This implies that the copper winding package can have a maximum thickness of $50 \mathrm{~mm}$. The minimum current density for generating the required magnetic field is then retrieved when assuming a single wire with a cross-section of $50 \mathrm{~mm}$ times $350 \mathrm{~mm}$. This leads to a current density of $2.6 \mathrm{~A} / \mathrm{mm}^{2}$ for a flux density of $160 \mathrm{mT}$. Due to the ohmic loss in the copper wires, such solenoids need adequate cooling [78]. For efficient cooling, we chose the solenoid to be wound from hollow square metal wires with an external cross section of $0.22 \mathrm{~mm}^{2}$. While the current is flowing through the copper edge of the wire, the dissipated heat can be very efficiently transported away by flowing cooling water through the hollow core of the wire. We have installed the magnet and characterized it. To generate a flux density of $B_{z}=160 \mathrm{mT}$, the required current is $176 \mathrm{~A}$. Note that, for an additional margin in magnetic field strength, the solenoid can provide up to $B_{z}=200 \mathrm{mT}$ when driving it with a current of $212 \mathrm{~A}$. The resistance of the wire package is about $0.07 \Omega$, thus the maximum power dissipation at $212 \mathrm{~A}$ is about $3.2 \mathrm{~kW}$. Using a water pressure of about 5 bar, which results in a water flow of about $1 \mathrm{l} / \mathrm{min}$ through the wire, this power can be easily cooled away. The magnetic field has been characterized using a magnetic field meter (F.W. Bell 615). The results are compared 
to the design values in Fig. 6.4b and a good agreement is found. As we have discussed above, the magnetic guiding is extremely robust against slight changes of the magnetic field. Thus, we can assume that, if the gun is properly aligned to the field lines, the electron beam should be transported with a high transmission efficiency and a low energy spread through the photonic crystal slab. For such alignment the standard procedure of mapping the electron beam position by a phosphor screen can be applied before placing the photonic crystal slab inside the vacuum vessel.

\subsection{Microwave design}

To provide amplification, a photonic crystal slab enclosed in a resonator is placed inside the solenoid, such that the electron beam travels through its center. While the design of the photonic crystal slab and the resonator are based on the numerical analysis given in chapter 3, we had to adopt three important modifications in the experiment (see Fig. 6.1b). At the upstream end, the electron beam enters through a hole with a diameter of $3 \mathrm{~mm}$ into the gain section. It is required to analyze the effect of this hole on the mirror function at the upstream end. Second, material losses will occur inside the photonic crystal slab which increases the laser threshold. Finally, back-reflections into the laser resonator might occur from the vacuum window which might change the threshold and tuning properties of the laser.

To allow the electron beam to enter the resonator, a hole is present in the upstream mirror. One might expect that a hole would considerably reduce the reflectivity of the mirror, but this is not the case. The hole in the mirror and the vacuum tube connecting the gun to the resonator are circular metallic waveguides. Electromagnetic waves can only propagate in such waveguides above a certain cut-off frequency. The inner diameter of the hole and the tube is relatively small, $3 \mathrm{~mm}$, which corresponds to a high cut-off frequency of about $64.9 \mathrm{GHz}[165]$. Below this cut-off frequency the waveguide reflects incoming radiation. As all frequencies that can be obtained with the described pFEL design are below $20 \mathrm{GHz}$, the entrance hole for the electron beam will not notable reduce the mirror reflectivity in the relevant spectral range.

The most important difference between calculations and experiment is certainly that in the experiments material losses are inevitable. Although metals only have weak losses at microwave frequencies, it is still important to quantify the losses. This enables to judge whether the associated increase of the laser threshold can be tolerated. The power loss in the photonic crystal slab, $P_{\text {loss }}$, can be estimated by calculating the current density, $J_{\text {surf }}$, induced by the tangential magnetic field, $\hat{\mathbf{H}}_{\mathbf{t}}$, at the surface of the metallic parts of 

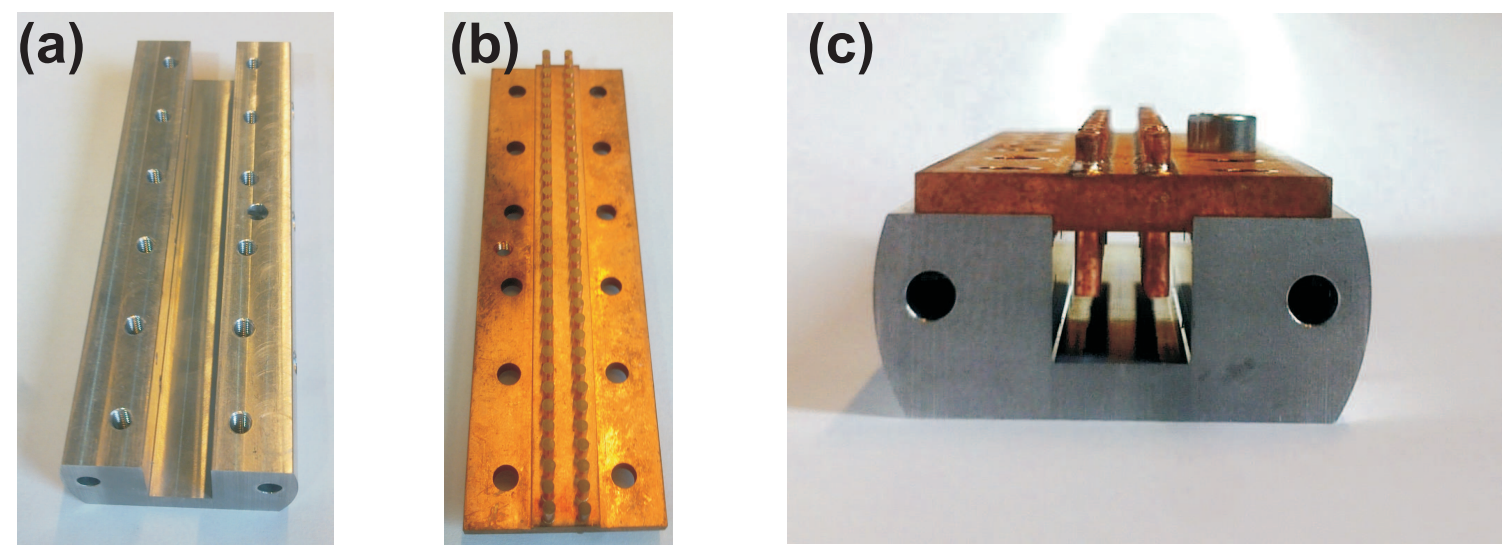

Figure 6.6: (a) Aluminium bottom plate of the manufactured photonic crystal slab, (b) Top plate of the photonic crystal slab, showing the copper rods soldered into the copper top plate, (c) Assembly of top and bottom plate to form the photonic crystal slab.

the photonic crystal slab [257].

$$
P_{\text {loss }}=\frac{R_{s}}{2}\left|J_{\text {surf }}\right|^{2}=\frac{R_{s}}{2} \int_{S}\left|\hat{\mathbf{H}}_{\mathbf{t}}\right|^{2} d \mathbf{S}
$$

Here the magnetic field $\hat{\mathbf{H}}_{\mathbf{t}}$ is taken from the eigenmode calculations for a lossless structure, and $R_{s}$ is the material dependent surface resistivity.

Choosing copper provides a very low surface resistivity, thereby reducing the losses. However, copper is a mechanically soft material. This imposes difficulties for manufacturing a photonic crystal. In addition, a high risk exists that certain parts of the photonic crystal slab become bent or distorted in the course of the experiment. Aluminum is in contrast to copper a much more rigid material, but its surface resistivity is about twice as high.

In view of the named material properties, combining both materials might give a mechanically rigid structure with still rather low losses. To find such combination, we compute the contribution by the different parts of the crystal to the surface integral in eq. (6.3). We find that the majority of surface currents is induced at the rod surface (about 80\%). To reduce the loss we have fabricated the crystal from two parts. The first piece (Fig. 6.6a) is a solid aluminum block that forms the bottom and the side of the waveguide for the crystal. The second piece (Fig. 6.6b) is a copper top plate holding the rods. The estimated loss per unit cell is about $0.12 \mathrm{~dB}$ for the complete assembly (Fig. 6.6c) which is found from eq. (6.3).

In order to estimate whether such loss still allows to overcome the threshold current with the given electron gun, we should compare it to the available gain. We estimate the 
laser threshold including material losses at the nominal beam energy of the electron gun of $14.2 \mathrm{keV}$. For this, we use the same procedure as described in section 3.4. However, now the losses are increased by the additional material losses. For a crystal length of 20 unit cells we find the threshold to be about $I_{t h}=70 \mathrm{~mA}$. Note that, we also reported in section 3.4 that the used linear model slightly underestimates the current threshold.

In the experiment we expect that we can easily compensate the effect of the losses with an increased length of the gain section compared to the limit of 20 unit cells in the numerical calculations. This number has only been chosen for the sake of reasonable short computation times. While increasing the length of the photonic crystal slab (and the number of unit cells) is straightforward in the experiment, we expect the main limitation to be the length over which the electron can be properly guided. When the selected gun is applied in a traveling-wave tube it can typically be guided over a length of $300 \mathrm{~mm}$ [251]. However, to have a variable length available in the experiments, we decided to have four separate crystal sections manufactured. The maximum total number of photonic crystal slab unit cells is 109, when inserting all four sections behind each other. For this case we calculate a strongly reduced threshold current of $I_{t h}=7 \mathrm{~mA}$, which is about three orders of magnitude below the maximum available beam current. In conclusion, even if we still have underestimated the laser threshold by an order of magnitude the laser should still easily turn on.

To characterize the generated emission it is transported through a vacuum window into a WR90 waveguide at atmospheric pressure. The task of the window is to prevent atmospheric gas to enter the vacuum vessel, while letting microwave radiation pass with minimum reflections. This is achieved by using the thin window concept. If the window is much thinner than the wavelength the phase advance during propagation through the window is nearly zero. As the reflection at the backside is phase shifted by $\pi$ and the amplitude is almost the same as the front reflection, the two reflections nearly cancel out. We use a vacuum window consisting of a circular sheet of kapton with a diameter of $40 \mathrm{~mm}$ and a thickness of $0.2 \mathrm{~mm}$ which is a $1 / 100$-fraction of the wavelength of the $\mathrm{TE}_{10}$ mode inside the waveguide at $16.0 \mathrm{GHz}$. To compute the reflection of such window we use a three-dimensional FDTD solver. Figure 6.7a shows the results for a frequency range of $14 \mathrm{GHz}$ to $16.5 \mathrm{GHz}$. Indeed, a very low reflection of about $1 \%$ is observed throughout the entire frequency range. The remaining power of about $99 \%$ is transmitted. At steadystate $1 \%$ of the output power amounts to about $15 \mathrm{~W}$, while the intracavity power is about $30 \mathrm{~kW}$. Such a small fraction of $0.5 \%$ can be expected to have a negligible effect on the laser threshold and frequency tuning [94, 258].

The components to realize such window are shown in Figure 6.7b. To seal the window, 
(a)

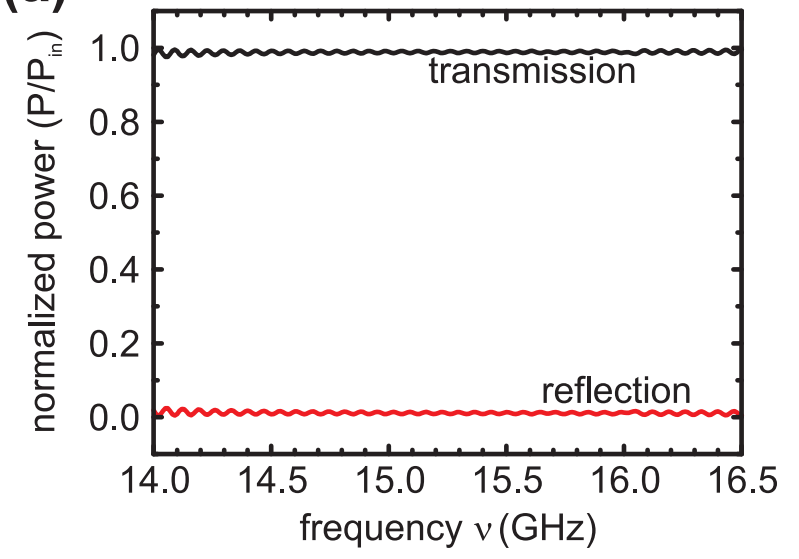

(b)

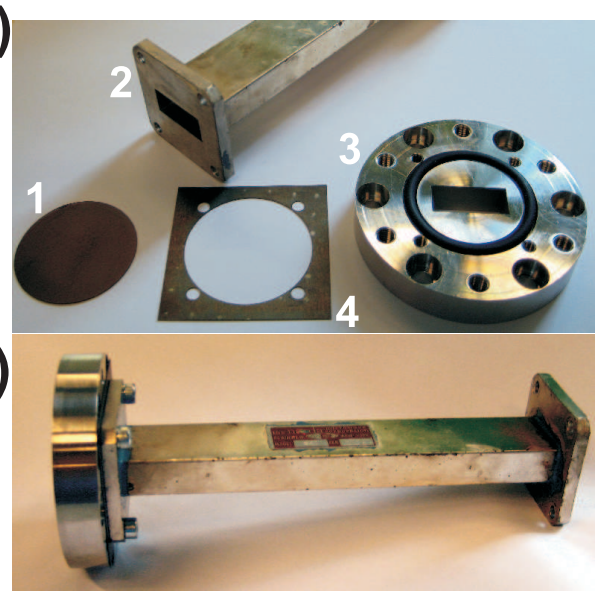

Figure 6.7: (a) Calculated reflection and transmission versus frequency for a kapton vacuum window with a thickness of about $0.2 \mathrm{~mm}$, (b) Components for realizing the thin waveguide window 1: kapton sheet, 2: atmospheric waveguide section, 3: final part of invacuum waveguide, 4: molybdenum sheet to hold kapton in place, (c) Complete vacuum window assembly.

the kapton sheet - labeled 1 in the Fig. - is sandwiched between the atmospheric waveguide and the final part of the in-vacuum waveguide section that holds an O-ring - labeled 2 and 3, respectively. To hold the kapton sheet in place when pressing it onto the O-ring it is surrounded by a molybdenum sheet - labeled 4 - with a thickness equal to the kapton sheet and a central hole in which the kapton sheets fits. The complete assembly is shown in Fig. 6.7c. This assembly has been successfully tested for its mechanical stability when one side is held at a vacuum pressure of about $1 \cdot 10^{-7}$ mbar. Finally, we note that it is extremely unlikely for electrons to hit and thereby destroy the functioning of the windows. Due to Coulomb repulsions the electron beam strongly diverges and all electrons should have hit the vacuum section waveguide shortly after the magnet (within about $40 \mathrm{~mm}$ ), while the distance to the window is more than $100 \mathrm{~mm}$.

\subsection{Conclusion and outlook}

This chapter described the most important components required to realize a pFEL. While an appropriate photonic crystal slab design and sufficient electron beam parameters have been identified by performing numerical calculations (see chapter 3.4), a number of challenges may occur when we actually want to realize these conditions in a laser. An electron beam has to be guided over many centimeters and be injected into a photonic crystal slab enclosed by a resonator to provide gain. All this has to occur in a ultra-high vacuum of 
less than $1 \cdot 10^{-7}$ mbar for operating the electron gun. This electron gun finally needs to be operated using high-voltages. Overall, we need to combine four different technologies, i.e., electron beam generation and transport, high-voltage technology, microwave engineering and vacuum technology.

A design which, we expect, can simultaneously meet those requirements has been presented. The electron gun is a commercial gun usually in use for traveling-wave tubes. A gridded electron gun requires a number of floating voltages, and the realization and design of the power supply have been discussed. The electrons are streamed through the structure via Brillouin guiding using a custom-designed electromagnet to facilitate the application of different beam voltages for frequency tuning of the device. An experimental characterization of the magnetic field shows good agreement with the calculated field strength. The calculated electron flow for such guiding field shows that we should be able to transport the beam through the photonic crystal slab as planned.

The microwave design is based on chapter 3. We discussed three modifications (with respect to the numerical modeling) which are required to perform the experiment and which might have an influence on the laser properties. First, a hole is present in the upstream mirror, which was shown not to affect the mirror function. Second, material losses increase the threshold current. However, as the laser design allows to increase the number of unit cells to more than one hundred, for providing an increased gain, the threshold current is estimated to be as low as $I_{t h}=7 \mathrm{~mA}$. This estimate is based on the linear model from section 3.4 which slightly underestimates the threshold. However, the expected threshold current is almost three orders of magnitude smaller than the maximum current provided by the selected gun. This should ensure that the laser reaches threshold, easily. The generated radiation will then be coupled out by a vacuum window, which we designed such that back-reflections into the laser remain limited to only $1 \%$. This value should be sufficiently small to exclude a significant influence on the properties of the laser, e.g., the laser threshold or the tuning characteristics.

In the near future, the described setup will be fully assembled to systematically investigate the operation of a pFEL for the first time. 


\section{7}

\section{Conclusions and outlook}

In this thesis, the first comprehensive study of photonic free-electron lasers (pFEL) has been given, based on a theoretical modeling via a systematic variation of its essential parameters. pFELs are based on the Čerenkov emission of many electron beams inside photonic crystals. The Čerenkov emission process can become stimulated if there exist certain modes for electromagnetic waves inside the photonic crystal having a longitudinal electric field component and a phase velocity that matches to the electron velocity. In this case the electric field generated from spontaneous Čerenkov emission can act back on the electrons to form electron bunches from the initially continuous stream of electrons. On average more electrons are decelerated than accelerated during the formation of bunches. The associated net reduction of kinetic energy in the electron beam is converted into electromagnetic field energy. Thereby, the velocity-matched amplitude of the electromagnetic wave grows and coherent radiation is generated by stimulated Čerenkov emission yielding laser oscillation. When tuning the electron velocity, the matching phase velocity is changed as well. Due to the dispersion of the photonic crystal mode this tunes the output frequency of the laser. As a pre-condition we limit the scope of this thesis to pFELs that use low-energy electron beams (about $10 \mathrm{keV}$ ). These electron energies can be produced by compact electron sources to achieve compact devices [63, 64].

The first central issue of this thesis is to achieve a comprehensive understanding of the 
amplification mechanism in pFELs, including the dynamic start-up of oscillation and the frequency tuning of the laser. The second central issue is to explore the power up-scaling properties of pFELs via pumping with multiple electron beams, as the latter is what enables also a frequency up-scaling of the laser. The third central issue is to prepare the realization of a pFEL in the microwave range to allow for a first thorough experimental investigation. Two chapters of this thesis are devoted to study the first two issues by numerically modeling pFELs either pumped with a single electron beam or with multiple beams. The first steps to realize pFELs for enabling also an experimental investigation of their properties is presented in two chapter as well. The results show that pFELs are promising for providing tunable high power lasers for the microwave and potentially for the $\mathrm{THz}$ regime.

The first set of numerical investigations in this thesis is aimed at comprehensively studying the laser properties of pFELs (chapter 3). For this purpose we investigate a simplified, prototype model of a pFEL for the microwave range, which is pumped by a single electron beam, with a beam energy of about $12.5 \mathrm{keV}$ and a beam current of $1 \mathrm{~A}$. The photonic crystal slab is $50 \mathrm{~mm}$ long and is formed by a periodic array of metallic rods placed inside a rectangular metallic waveguide.

We explain that stimulated emission and laser oscillation can be well described via the following working principle. As a response to electrons passing the metallic rods multiple wavelets are emitted from the photonic crystal. Constructive interference of these wavelets can be achieved by designing the photonic crystal to offer a suitably low phase velocity that roughly matches to the velocity of the electrons. Feedback from this emission upon the electron beam leads to an electron bunching with a spatial period matching the spatial period of the field that caused it. This yields stimulated emission, as also found in other types of FELs. In order to investigate these described dynamics in more detail, and to allow also quantitative predictions, we have modeled the linear and nonlinear properties of pFELs with a rigorous numerical method, the particle-in-cell (PIC) method. Although such detailed modeling can become rather tedious, it has allowed us to understand and analyze the physical dynamics of electromagnetic field amplification and the dynamics of the electron beam leading to steady-state oscillation. Further, for the specific pFEL under investigation we have studied the main laser characteristics. These are the dynamical growth of the electromagnetic field, the spatio-temporal electron beam dynamics, the laser current threshold, the growth of the steady-state power with the pump current and the tuning of the operation frequency with the electron velocity.

We find that, after turning on the electron beam, initially the continuous stream of electrons along the metallic rods generates spontaneous emission. The photonic crystal 
slab we have chosen offers a particular mode which is velocity-matched at a low spatial harmonic and also provides a strong longitudinal electric field component. This allows the initial spontaneous emission in this particular mode to induce the onset of electron bunching. Although the initially induced electron bunching is weak, it nevertheless subsequently enhances phase-matched emission into that photonic crystal mode. This means that the initial electromagnetic field is amplified in phase, which is a clear signature of stimulated emission. In turn the growing amplitude of that mode enhances the bunching of the electrons. If the electron beam is sufficiently strong the gain provided by this feedback mechanism exceeds the losses and exponential growth over many orders of magnitudes sets in. Eventually, the gain saturates with the following dynamics. When the electrons amplify the electromagnetic wave their average kinetic energy reduces, which brings the electrons out of velocity-matching with the electromagnetic wave. This reduces the growth of the field amplitude until a steady-state output is reached, where the saturated gain balances the losses. At steady-state, the output power is $1.5 \mathrm{~kW}$ and the output spectrum typically comprises of a single frequency. For our specific example this frequency is around $16 \mathrm{GHz}$ and the laser output shows a resolution limited bandwidth of about $2 \mathrm{MHz}$. By varying the beam current we found that the threshold current is about $I_{t h}=140 \mathrm{~mA}$ and that the output power scales linearly with the applied pump current. We observe that, by changing the electron beam energy from $10 \mathrm{keV}$ to $15 \mathrm{keV}$, the laser frequency tunes according to the expected velocity-matching between photonic crystal slab and electron beam.

While the described PIC calculations are very valuable for investigating the properties of a specific pFEL to great detail, such calculations are also very time consuming and tedious. To address this inherent problem we also compared the PIC calculations to the simple linearized gain model of Pierce, summarized in section 2.6. A satisfying agreement between both descriptions was found. For the chosen example of a pFEL, the small-signal gain per wavelength was found to be as close as $0.2 \mathrm{~dB}$ to the result of the extensive PIC calculations. Also the predicted laser threshold of $I_{t h}=37 \mathrm{~mA}$ is reasonably close to the laser threshold found in the PIC calculations $\left(I_{t h}=140 \mathrm{~mA}\right)$. In the future, the good agreement for the small-signal gain and the laser threshold from the two approaches will allow a quick assessment of the suitability of a larger variety of different photonic crystals geometries for the application in pFELs.

In the following chapter 4 , we discuss the options for power and frequency scaling in pFELs. As has been recalled in section 2.4, photonic crystals are freely scalable to any desired frequency. This means that, when the geometrical size of the photonic crystal is scaled down with a certain factor, $s$, the frequency of its modes is increased by the same 
factor $s$, while the relative field distribution of the modes remains unchanged. Based on this simple scaling relation, one might think frequency up-scaling of the laser frequency in a pFEL is straightforward. However, it turns out that the additionally required scalability of the electron beam is fundamentally limited, such that the pump power provided by the electron beam reduces. When downscaling the characteristic length scale of the photonic crystal with the factor $s$, the cross sectional area of the vacuum channel in the crystal through which the single electron beam streams inevitably reduces. The area approximately reduces with the square of $s$. Thus, attempting to maintain a constant pump current - for achieving a constant output power - would cause the current density to increase quadratically with the scaling factor $s$. Eventually, this is not possible anymore, due to the mutual Coulomb repulsion of the electrons in the pump beam. The consequence is that decreasing the size of the photonic crystal reduces the pump power. Beyond a certain maximum output frequency the possible pump current drops below the threshold current. Thus, the laser will not start-up.

Photonic crystals offer a viable approach for achieving frequency scaling using the following power scaling principle. Photonic crystals naturally provide many vacuum channels in parallel. Instead of increasing the current density in a single electron beam for power scaling, it appears possible to drive the laser by multiple, spatially separated electron beams. This avoids stronger Coulomb repulsion when increasing the total beam current and thereby the pump power. In other words, we suggest for the purpose of frequency scaling to compensate the pump current loss per beam by increasing the total number of beams. Accordingly, it should be possible to maintain a constant pump and output power.

At the basis of frequency scaling in pFELs are thus the properties of power scaling via multiple pump beams. To investigate these properties at an example, we have studied how the quantity and quality of the output from a specific pFEL is affected by increasing the number of electron beams, in our case in steps from one to seven beams. For this investigation we increased the number of transverse periods of the photonic crystal slab. This creates the required, large, pump volume for streaming many electron beams. Note that, in other lasers such increase of the transverse size of the laser mode volume typically leads to oscillation in multiple transverse modes, which is equivalent to a loss of brightness of the laser output. However, when calculating the output in our pFEL with a wide photonic crystal slab pumped with seven beams, we find that the lowest (fundamental) transverse mode dominates and contains more than $95 \%$ of the total output power, which amounts to about $8 \mathrm{~kW}$.

In the studied case, this observation is most likely caused by the fact that the pFEL is 
pumped by mono-energetic electron pump beams, which can be considered as providing mode competition for the gain in analogy to homogeneous gain broadening. We verified this explanation by comparing the small-signal gain for the various, potentially oscillating modes using the linear model. We find that the small-signal gain is highest for the dominant fundamental mode. The reason for this is that all higher-order photonic crystal modes have field nodes which lower the spatial overlap with the pump beams. While it might eventually not be the fundamental photonic crystal mode that dominates the small-signal gain, it will be always dominated by a certain single mode. This single mode is than expected to dominate the stimulated emission at steady-state as well. Using a suitable diffractive optical element a single mode in the output waveguide can always be converted into a single frequency $\mathrm{TEM}_{00}$ mode in free-space to obtain the highest possible brightness for a given power and frequency.

In order to find an approximate power scaling law for more than the limited number of seven beams we could investigate with PIC calculations, we compare the numerical results with scaling based on qualitative arguments. The numerical calculations show, that each pump power increment via adding pairs of adjacent beams results in a further increase of the output power but with a slightly lower power increment. Hence, the output power scales somewhat lesser than linear with the number of beams. We investigated whether this deviation from a purely linear scaling is due to the decreasing electric field strength along the transverse coordinate, which would reduce the interaction with the electron beams. The power scaling law we retrieved using an effective dielectric model for the photonic crystal slab shows a similar tendency as that found in the PIC calculations.

The observed power scaling in pFELs achieved with multi-beam pumping could be the key for increasing the operating frequency of the pFEL, which is subject of future investigations. We envision that, eventually, the current might be distributed over hundreds or thousands of beams, for example provided by field-emitter arrays [63, 187]. Although the losses of metallic photonic crystals are certainly higher at a higher frequency we expect that it will be possible to pump a single-mode of a pFEL at $\mathrm{THz}$ frequencies and generate several watts of output power with a high spectral and spatial brightness.

The remainder of the thesis is aimed to prepare the realization of a microwave pFEL to allow for the first time a systematical comparison and verification of the expected properties of a pFEL by experiments. As we have discussed throughout the previous paragraphs, the key element for providing amplification is the photonic crystal. More specifically, it is the longitudinal electric field component of the photonic crystal that is present at the position of the electron beams. This field component determines the absolute strength of amplification. Thus, it is important to experimentally characterize this field compo- 
nent for photonic crystals that are considered for pFELs. However, measurements of field components well inside a photonic crystal have never been undertaken. In chapter 5 we present and demonstrate a method how to map the absolute value of individual electromagnetic field components inside photonic crystals. The method relies on measuring the change in resonance frequency when the photonic crystal is placed inside a resonator and the field inside the photonic crystal is perturbed by a sub-wavelength scatterer. In our experiments a spherical scatterer is applied to measure the longitudinal electric field component in a specific photonic crystal slab, in which this field component dominates. We observe good agreement between measured and calculated electric field strengths without using any adjustable parameters in the calculations.

Notably, this method might not only be interesting for the particular mapping of field distributions inside photonic crystals for the use in pFELs, which are designed for operation in the microwave and $\mathrm{THz}$ range. We envision that, this method could even be applied in the near infrared or optical domain by scaling down the setup. Instead of a bead on a string we propose mounting a metallic or dielectric scatterer on one end of a stiff carbon nanotube whose other end is mounted on the tip of an atomic force microscope. The scatterer is then moved by moving the atomic force microscope tip. Mounting a carbon nanotube on an atomic force microscope has been demonstrated [244]. Further, atomic force microscopy allows a sufficiently high spatial resolution, as demonstrated by recent measurements where an emitter directly mounted to an atomic force microscope has been used to map the emitter lifetime around a single nanorod [245]. Combining these techniques, it should be possible to measure the shift in resonance frequency in the transmission spectrum in order to determine the absolute field strength inside accessible air voids of a photonic crystal at optical frequencies.

Chapter 6 of this thesis presents a specific technical design for realizing a pFEL in steady-state operation at microwave frequencies. Previous attempts to realize pFELs were based on high electron energies $E_{e l}>200 \mathrm{keV}$, which are at least an order of magnitude beyond what we base our approach on. Those experiments also used a huge beam current beyond $I>1000$ A [246, 247], which is at least three orders beyond what we expect as threshold current. These experiments have failed to achieve steady-state operation, because the applied megawatt pumping could not be provided with pump parameters that are constant over times characteristic for the laser dynamics. Consequently, the output and its properties are hard to control and difficult to characterize thoroughly. This makes a systematic comparison between theory and experiment effectively impossible.

Unlike previous attempts, our pFEL design is based on well-known technological knowhow taken from microwave tubes. This means that a standard electron gun operated at 
a beam energy of about $10 \mathrm{keV}$ and a beam current of about one ampere is applied. This should allow an excellent control of the experimental conditions and parameters to enable an accurate comparison to theory and a systematical experimental study. While we base the fundamentals of the design on the numerical calculation of chapter 3 , that model is of course an idealization. A number of modifications are required to enable the experiment due to technical limitations. First, in the modeling, a certain area of the perfect reflecting mirror emits the electron beam for numerical convenience, and the beam radius is held approximately constant throughout the rest of the crystal volume via the application of a strong, homogeneous magnetic field. While standard electron guns provide such beam properties, a proper guiding scheme is to be designed for transporting the beam with a constant diameter through the crystal. In addition, standard guns can also not be integrated into the photonic crystal, but the electron beam must enter the gain section through an injection hole in one of the resonator mirrors. Second, for proper operation of an electron, gun an ultra-high vacuum of better than $1 \cdot 10^{-7}$ mbar is required. Third, to operate the electron gun high voltages are required. The generation and handling of high voltages must be properly designed, for example, to avoid breakthroughs. Fourth, inside a real photonic crystal losses will inevitably occur and raise the laser threshold. Fifth and finally, for a characterization of the output the generated radiation is to be separated from the electron beam and to be coupled out from the vacuum vessel via a low-reflective vacuum window. Overall the proper design of a pFEL requires to combine four different technologies, i.e., electron beam generation and transport, high-voltage power supplies, microwave engineering and vacuum technology.

Our design to fulfill these requirements is based on a commercial dispenser electron gun that is usually applied in traveling-wave tubes. This particular electron gun provides up to $2 \mathrm{~A}$ of beam current at its nominal beam energy of $14.2 \mathrm{keV}$. For studying the frequency tuning of a pFEL the beam energy can be varied in a range of $10 \mathrm{keV}$ to $15 \mathrm{keV}$. Proper operation of the electron gun requires a vacuum of better than $1 \cdot 10^{-7}$ mbar to avoid the destruction of the cathode via so-called poisoning. To avoid excessive demands on the power supplies and the thermal laser design, the electron gun is equipped with a grid electrode for pulsing the electron beam. The gridded gun needs to be supplied with several floating voltages, and the realization and design of the power supply has been presented. The Coulomb repulsion among the electrons inside the beam require an external guiding force to prevent degradation of the gain through electrons being intercepted by the crystal and to avoid an increased energy spread of the electron beam. In addition, for studying frequency tuning, the application of various beam energies is required. A guiding technique that allows these conditions to be implemented most easily is Brillouin 
flow guiding with a solenoid electromagnet. We presented the careful design of the electromagnet and the associated electron flow, as well as the realization of the magnet. A good agreement between the calculated magnetic field strength and the measured field strength was found. In numerical calculations the electron flow through the magnet has been shown to be rather robust versus variations of the magnetic field strength. The magnet should therefore enable the transport of the electron beam with a high transmission efficiency and low energy spread through the photonic crystal slab as planned.

For the microwave design of the laser we discussed three key modifications, with respect to the numerical modeling in chapter 3. These modifications are crucial for enabling the experiment, but could potentially influence the laser properties. First, a hole is present in the upstream mirror for injecting the electron beam. Our estimates show that this should have no impact on the mirror function. Second, material losses are present in a real structure which inevitably increases the threshold current. However, based on the procedure described in section 3.4 we estimate that the threshold current can be as low as $7 \mathrm{~mA}$ when using the maximum photonic crystal slab length of over one hundred unit cells. While we found that the applied procedure slightly underestimates the threshold current, the expected threshold is almost three orders of magnitude smaller than the maximum current provided by the selected gun. Thus, we are confident that the laser easily reaches threshold and laser oscillation will be observed. Third and finally, the radiation is coupled out via a vacuum window in order to characterize the generated radiation outside of the vacuum vessel. Such a window typically gives rise to reflections back into the laser resonator, which might alter the laser threshold and the frequency tuning characteristics. Using a thin window, the calculated reflections into the laser resonator are limited to about $1 \%$. This value should be small enough to be of negligible influence for the laser properties.

Taking into account all the differences between the model presented in chapter 3 and the experimental design in chapter 6, we expect that, after fully assembling the setup, the first systematic experimental analysis of pFELs becomes possible.

In conclusion, the theoretical modeling presented in this thesis and the subsequent experimental demonstration and laser operation based on this thesis may become the key for the realization of a new family of compact and high-power laser sources for the microwave to the $\mathrm{THz}$ spectral range. 


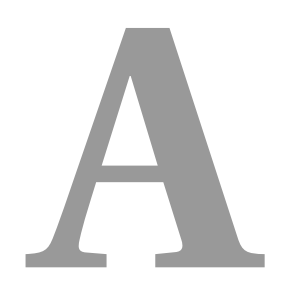

\section{Laser resonators for pFELs}

In any laser oscillator, the presence of a feedback resonator and its properties strongly impacts on the laser operation. For example, considering a most commonly used solidstate laser medium, Nd:YAG [196], at least eight entirely different frequency ranges are emitted, depending on the spectral properties of the resonator mirrors [169]. However, the resonator design also determines the spatial properties of the generated output, as can be seen when the laser beams obtained from stable resonators are compared with those from unstable resonators $[169,259]$. An experimental investigation of the spatial and spectral emission internal to a laser resonator is typically only possible via a characterization of the light outside the resonator. Due to limitations in the software used to perform the PIC calculations in chapter 3 and chapter 4 , we encounter the same limitation in analyzing the results of the numerical calculations. Thus, for drawing appropriate conclusions from the PIC calculations, it is obvious that the influence of the out-coupling on the spatial and spectral composition of the light must be known.

The properties of the resonator element used for out-coupling are generally dictated by the electromagnetic boundary conditions imposed by the interfaces of that element. For instance, when using the interface to a material with an increased refractive index as the out-coupling element, the partial reflection and output coupling from that interface can be calculated via the well-known Fresnel formula [260]. While the light frequency remains 
unchanged upon reflection and transmission, the Fresnel formulas show that, generally, not only the amplitude, but also the orientation of the electromagnetic field vector might be affected by the interface. For example, in case of Brewster reflection, only a certain polarization is reflected. Thus, even in a simple case of a plane wave impinging on a plane interface, the orientation of the reflected and transmitted electromagnetic field vectors are different. At the transition between a photonic crystal slab and the subsequent output waveguide, such as that considered in our examples of pFELs, the situation becomes even more complex. Unlike in a plane wave, the field strengths also vary transversely to the propagation directions.

Thus, we can expect that at an interface between a photonic crystal and a waveguide, such as that found in our pFELs, the reflection and the transmission for an impinging electromagnetic wave are rather complex to predict. For example, assume a single photonic crystal mode, i.e., a certain complex transverse field shape, propagates inside a crystal and reaches the interface to a waveguide. At the interface part of the mode is reflected and a part of the eigenmode is transmitted. The exact spatio-temporal form of the field that is transmitted is determined by the precise boundary conditions. Because the spatial shape of the eigenmodes in the photonic crystal differs fully from the shape of the eigenmodes in an empty waveguide, the prediction of the transmission into the waveguide is far from obvious without performing detailed calculations. Therefore, even if only a single photonic crystal mode impinges on the interface, the light transmitted into the waveguide will generally excite several different waveguide modes. Due to the software used for the PIC calculations, the pFEL output field is already decomposed into the modes of the output waveguide when sampling them at the output port. Thus, if we are unable to relate the observed mode content at that plane to the mode content inside the photonic crystal, we are also unable to clarify any effects such as transverse mode competition inside the pFEL.

At first sight the intricate transmission seems only to complicate the numerical investigations of a pFEL. However, the amount of transmission also impacts directly on the operation of the laser. Assume that the interface between a photonic crystal and a waveguide forms one mirror of a resonator, as in our pFELs. The losses of the resonator become then dependent on the spatial and spectral properties of the transmission at the interface (out-coupling), which directly affects the laser threshold. Next to these so-called active losses from the out-coupling at the resonator mirrors, passive losses, such as material losses, add to the total loss of a resonator. However, for the pFEL we have neglected material losses throughout the numerical calculations. Further, in both lasers we study in this thesis, one of the pFEL mirrors is a perfect mirror. This means the out-coupling 
from this mirror is $T=0 \%$ and, accordingly, its reflectivity is $R=100 \%$. Thus, the resonator losses are entirely determined by the transition between the photonic crystal slab and the output waveguide.

In this appendix we present a study of the effects at this transition between the photonic crystal slab and the output waveguide in a pFEL. In the first section of this appendix, we study whether the output waveguide modes are correlated to single modes inside the photonic crystal. In the second section we show how we can deduce the effective reflectivity and thus out-coupling at the named interface.

\section{A.1 Transmission at the interface}

As is clear from the introduction, we need to relate the mode content at the output port to the modes in the photonic crystal. Only then the observed behavior at the output port can be used to describe the dynamics of the modes inside the photonic crystal.

It is possible to realize that, at the same frequency, only a single mode is allowed to propagate in both the empty waveguide and the photonic crystal slab. The coupling between the two can then be expressed by a simple transmission and reflection coefficient. Such is the case for the pFEL studied in chapter 3. However, for the multi-beam pFEL described in chapter 4, several transverse modes can propagate in the crystal and in the waveguide. Figure A.1 shows the dispersion of the photonic crystal modes for the pFEL from chapter 4 together with the cut-off frequencies of the lowest order waveguide modes in the output waveguide. It is clearly seen that for the operating frequency of this specific pFEL, about $16 \mathrm{GHz}$, multiple modes can propagate inside the photonic crystal slab and inside the waveguide.

A priori, it is impossible to know whether a certain photonic crystal mode exclusively couples to a certain waveguide mode. Coupling depends on the specific boundary conditions at the interface [261-263]. However, certain conclusions can still be drawn if there is a matching spatial symmetry between the geometry of the waveguide and the crystal. Specifically, in our case both systems are symmetric to the same $y z$-plane that goes through the center of both the waveguide and photonic crystal slab. Thus, the eigenmodes of both systems can be classified with respect to their symmetry to this plane. If the field distribution of a crystal mode is mirror symmetric to the symmetry plane, it can couple only to waveguide modes that are also mirror symmetric. A similar argument applies to modes that are anti-symmetric to the named plane. However, any further conclusions require computation of the precise coupling between photonic crystal and waveguide modes at the interface. 


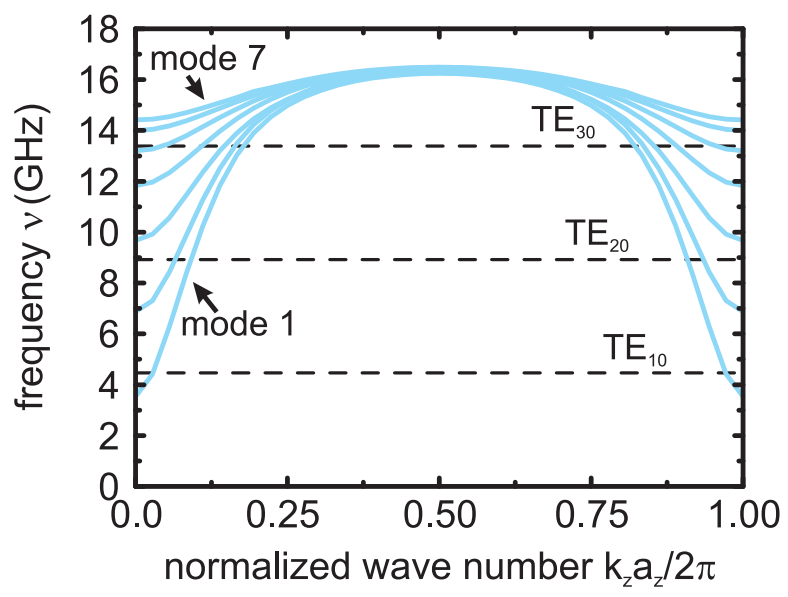

Figure A.1: Band diagram of the photonic crystal slab and cut-off frequencies of the lowest order modes in the output waveguide for the pFEL shown in Fig. 4.1 indicating that multiple modes exist in both systems.

Computing the transmission of photonic crystal modes into the waveguide first requires the selective excitation of photonic crystal modes in the resonator. This requires the excitation of an infinite summation of plane waves with appropriate relative amplitudes and proper phasing, see eq. (2.22). While this is not impossible, typical three-dimensional electromagnetic wave solvers do not allow such excitation [141, 208]. Typical software packages usually allow only the excitation and detection of single waveguide modes in a certain reference plane (input, output). To avoid a time consuming development for a direct excitation of photonic crystal modes, we first performed a basic check whether specific single waveguide modes would already enable the excitation of a single photonic crystal mode. If this check turns out to be positive we could subsequently study the outcoupling of this single photonic crystal mode using this simple excitation. Luckily, this check did indeed turn out to be positive. The model we used to compute the described case is shown in Fig. A.2. A rectangular waveguide is attached to both sides of the photonic crystal slab for the multi-beam pFEL. At one side, a certain waveguide mode is injected, and we study the power at the input and output decomposed into waveguide modes. For performing these calculations, a frequency domain method is used [141].

In the PIC calculations we have observed only radiation below $16 \mathrm{GHz}$, accordingly we limit the frequency domain computations to waveguide modes which can propagate below that frequency. From Fig. A.1 we observe that the waveguide allows three eigenmodes: $\mathrm{TE}_{10}, \mathrm{TE}_{20}$ and $\mathrm{TE}_{30}$ [165]. From our previous discussion of reflection and transmission at interfaces we can expect, that when injecting a single mode, the other propagating modes are excited via mode conversion at the interface. For example, when exciting the $\mathrm{TE}_{10}$ 


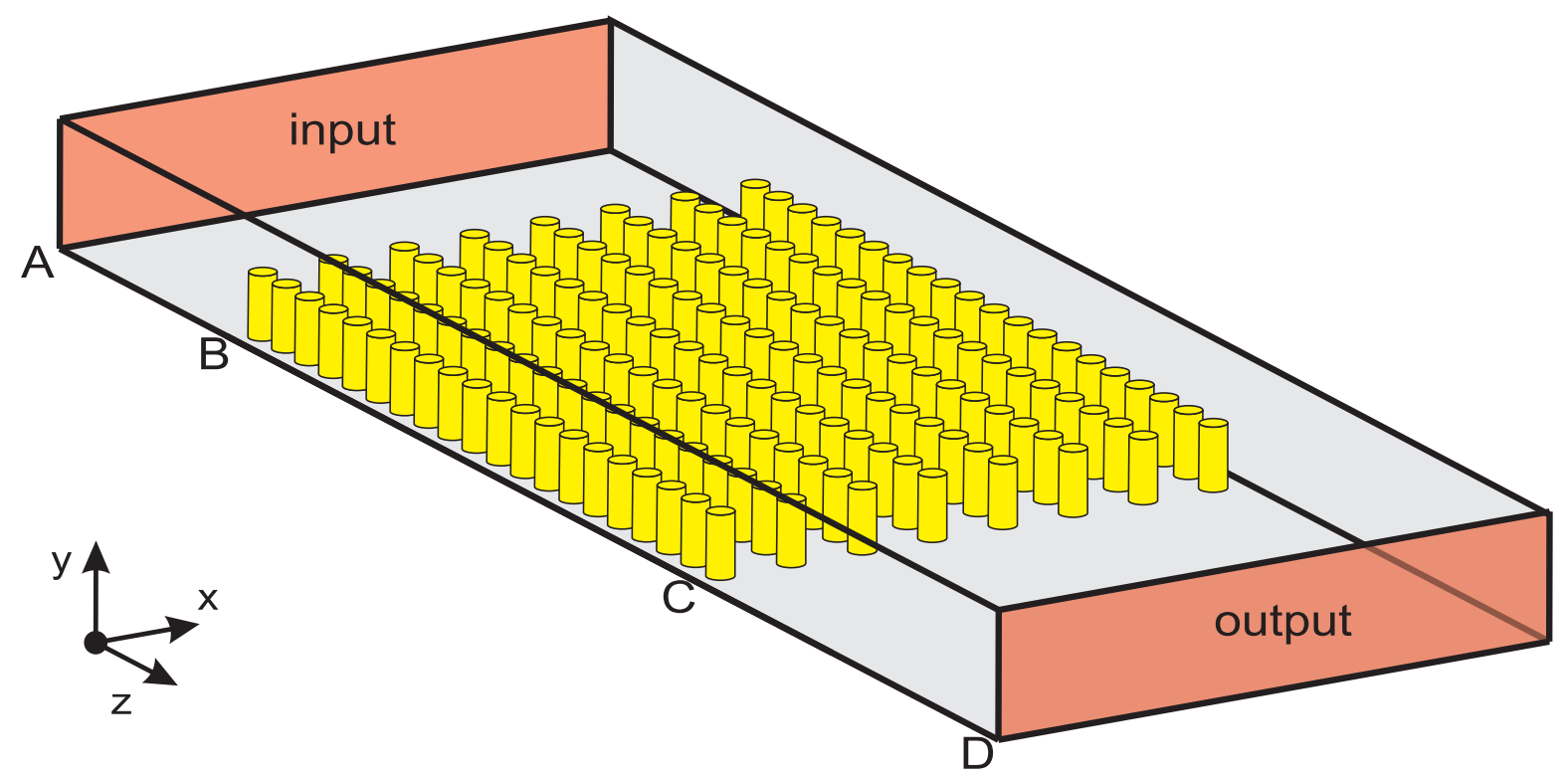

Figure A.2: Numerical model for computing the transmission and reflection of the photonic crystal slab modes into the rectangular waveguide modes and vice versa. At the input, a certain waveguide mode shape is excited and the reflection and transmission into the excited mode and also into the other two propagating modes are computed.

mode at the input, we can expect power in all three propagating waveguide modes at the input and output, i.e., $\mathrm{TE}_{10}, \mathrm{TE}_{20}$ and $\mathrm{TE}_{30}$. The same argument holds for excitation with the $\mathrm{TE}_{20}$ and $\mathrm{TE}_{30}$ modes. In the following, detected reflection and transmission in the excitation mode is named self-reflection and self-transmission. Detected reflection and transmission in other than the excited mode are named cross-reflection and crosstransmission. In order to find the spectrum of both types of transmission and reflection, we sweep the frequency from $15.0 \mathrm{GHz}$ to $16.0 \mathrm{GHz}$ in steps of $1.3 \mathrm{MHz}$.

When analyzing the reflection and transmission for an excitation with $\mathrm{TE}_{10}, \mathrm{TE}_{20}$, and $\mathrm{TE}_{30}$ input, we find that the input mode is not converted into other waveguide modes via in-coupling into or out-coupling from the photonic crystal slab. Quantitatively, the corresponding coefficients for cross-reflection and cross-transmission are ten orders of magnitude lower than the three coefficients for self-reflection and self-transmission. Therefore, mode conversion at the interface can be neglected. The self-transmission for the three input modes are shown in Fig. A.3. We observe that, for each input mode, the transmission shows a distinct set of single-peaked resonances. This indicates that reflection at the interface between the slab and the waveguide spectrally restricts the build-up of the electromagnetic field inside the photonic crystal. Only electromagnetic fields with wave numbers $k_{z}$ that correspond to a phase advance of a multiple of $2 \pi$ per resonator round-trip can build up, see also section 5.5. These sets of wave numbers 


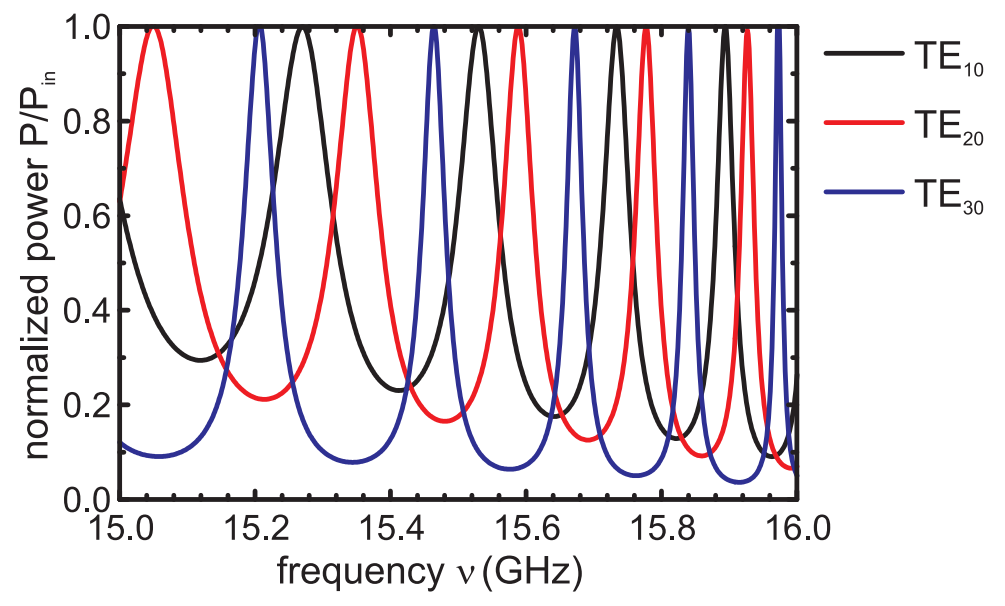

Figure A.3: Self-transmission spectrum for the input waveguide mode $\mathrm{TE}_{10}, \mathrm{TE}_{20}$, and $\mathrm{TE}_{30}$.

are determined by the resonator length and are, therefore, the same for any transverse photonic crystal mode, see eq. (5.4). However, the associated frequencies for these set of wave numbers depend on the dispersion of the photonic crystal mode. In our case the dispersion for mode 1 to mode 3 is different. The fact that each input mode leads to a distinct set of resonances, where not a single resonant frequency is identical to any other input mode is a strong indication that each input mode excites a single photonic crystal mode.

In order to identify this photonic crystal mode, we calculate the field distribution inside the crystal when injecting a certain input mode with one of its resonant frequencies. Figures A.4 a-c show the electric field component $\hat{E}_{y}$ inside the photonic crystal for a $\mathrm{TE}_{10}, \mathrm{TE}_{20}$, and $\mathrm{TE}_{30}$ input. Figures A.4 d-f show the $\hat{E}_{y}$ field distributions found when analyzing the unit cell of the photonic crystal with an eigenmode solver. It is obvious that figures in the same row show nearly the same field distributions. Thus, we find that the photonic crystal mode 1 is almost exclusively excited by a $\mathrm{TE}_{10}$ input, mode 2 by a $\mathrm{TE}_{20}$ input and mode 3 by a $\mathrm{TE}_{30}$ input.

In order to investigate how the out-coupling of these modes into the waveguide occurs, we recall that no cross-transmission is found in the calculations. Hence, each photonic crystal mode also couples out into the same, single waveguide mode that excited it. In conclusion, this result means for the analysis of the results from the PIC calculations, that the observed power growth of each waveguide mode can be unambiguously associated with the power growth of a single photonic crystal mode. Further, this means that by studying the relative power changes of different waveguide modes at the output port we can investigate the competition of photonic crystal modes inside the laser. 
(a) $\mathrm{TE}_{10}$ mode input, $v=15.89 \mathrm{GHz}$

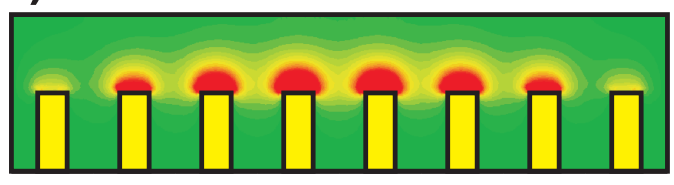

(b) $\mathrm{TE}_{20}$ mode input, $v=15.84 \mathrm{GHz}$

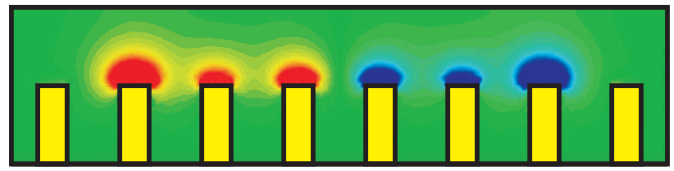

(c) $\mathrm{TE}_{30}$ mode input, $v=15.92 \mathrm{GHz}$

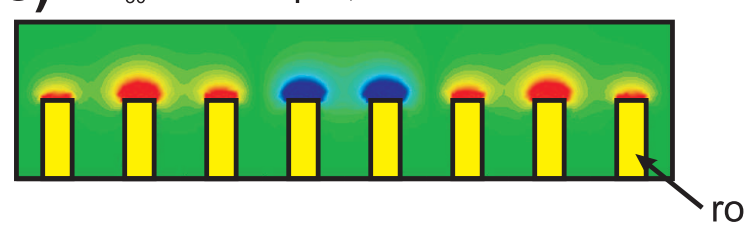

(d) slab mode $1, v=15.89 \mathrm{GHz}$

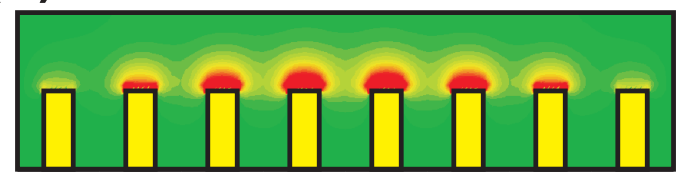

(e) slab mode $2, v=15.84 \mathrm{GHz}$

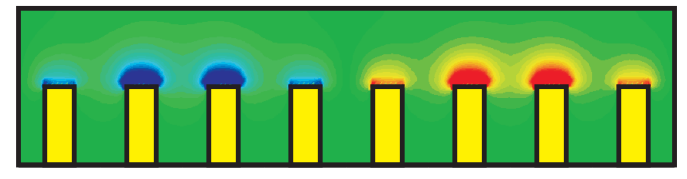

(f) slab mode $3, v=15.92 \mathrm{GHz}$

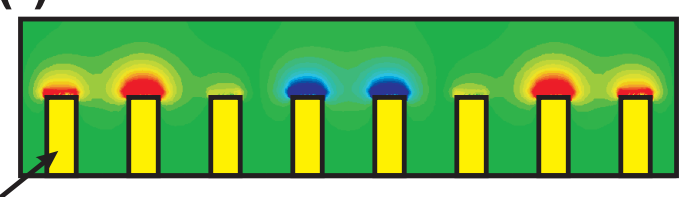

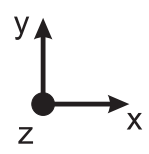

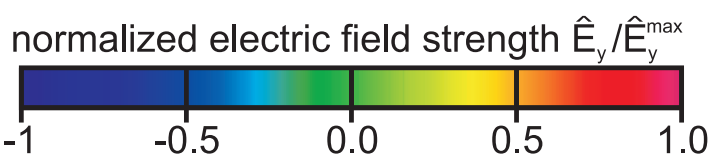

Figure A.4: Electric field component $\hat{E}_{y}$ inside the photonic crystal slab at a cross section ( $x y$-plane) through the center of the rods. In the left column $(\mathrm{a}-\mathrm{c})$, the electric field component $\hat{E}_{y}$ computed for an waveguide input by a $\mathrm{TE}_{10}, \mathrm{TE}_{20}$, and $\mathrm{TE}_{30}$ mode, respectively. In the right column $(\mathrm{d}-\mathrm{f})$, the electric field component $\hat{E}_{y}$ for the photonic crystal mode 1 , mode 2 and mode 3 computed at the same frequency as used in a to c, but obtained by an eigenmode analysis of the unit cell of the photonic crystal. 

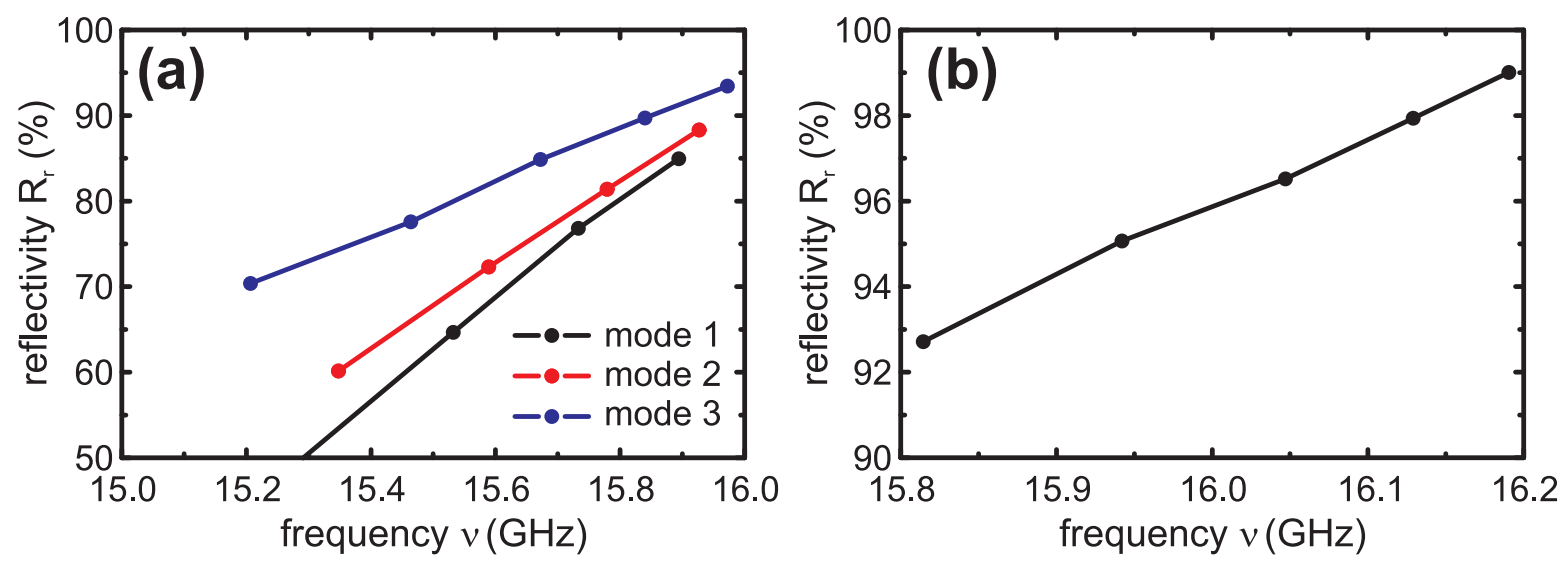

Figure A.5: (a) Reflectivity versus frequency of the interface for the multi-beam pFEL, (b) Reflectivity versus frequency for the single-beam pFEL.

\section{A.2 Reflection at the interface}

In the previous section we have shown that, at the boundary between our specific photonic crystals and a rectangular waveguide, a single mode of the crystal couples to a single mode of the waveguide. However, we did not quantify the out-coupling of a single photonic crystal mode at the interface, or in other words, the reflectivity at the interface. This quantification is important for the calculation of the threshold pump power of a pFEL as presented in section 3.4. In addition, such quantification is important as it simplifies the discussion of mode competition because it allows to compare the gain per wavelength of the modes instead of the gain per round-trip (see 4.4). In this section we present how to compute the reflection coefficient from the quality factor $Q$ of a longitudinal resonance.

As we neglect material losses and because both the resonator mirrors in the calculations of the last section have the same reflectivity $R_{r}$ for a resonance $r$ we find that

$$
R_{r}=\exp \left(-\pi \nu_{r} \frac{\tau_{r e s}}{Q_{r}}\right)
$$

with the round-trip time equals $\tau_{\text {res }}$

$$
\tau_{\text {res }}=\frac{2 L_{r e s}}{v_{p h}} .
$$

Here, we used that $Q=\nu_{r} / \nu_{r, \text { FWHM }}$.

We determine the quality factor $Q$ of each transmission resonance from Fig. A.3 and find the phase velocity from the dispersion in Fig. A.1. Using eq. (A.2) we calculate the reflectivity. Figure A.5a shows the results for the three lowest transverse modes of 
the multi-beam photonic crystal versus frequency. We observe that the the reflectivity increases towards $16 \mathrm{GHz}$. This is expected, as it is well-known that the reflectivity at an interface generally increases with the group velocity-mismatch between the modes at an interface [62]. While the group velocity of the waveguide modes increases towards $16 \mathrm{GHz}$, the group velocity of the crystal modes decreases resulting in a bigger group velocitymismatch. Most importantly, the reflectivity at the operating frequency of $15.87 \mathrm{GHz}$, which we observed in section 3.4, for the multi-beam pFEL differs for the three modes by only $5 \%$. Thus, we can simplify the discussion of mode competition to analyzing the gain per wavelength instead of the gain per round-trip.

For computing the reflectivity of the single beam pFEL we have applied the same method and the results are shown in Fig. A.5b. Once more we find the same trend: an increasing reflectivity when the frequency approaches $16 \mathrm{GHz}$, as the group velocity mismatch increases between waveguide and photonic crystal. We find that the reflectivity is about $95 \%$ around $15.9 \mathrm{GHz}$, which is used for determining the laser threshold in section 3.4 . 



\section{Bibliography}

[1] M. Weidhorn, The person of the millennium: the unique impact of Galileo on world history (iUniverse Inc., 2005).

[2] G. Galilei, Siderius nuncius (Venetiis: Apud Thomam Baglionum, 1610). http://www.rarebookroom.org/Control/galsid/index.html

[3] C. K. Kao, Les Prix Nobel. The Nobel prizes 2009 (The Nobel Foundation, 2010), chap. Sand from centuries past: send future voices fast, pp. 71-81.

[4] M. Castells and G. Cardoso, The network society: from knowledge to policy (Center for transatlantic relations, 2005).

[5] W. C. Röntgen, "Über eine neue Art von Strahlen," in Aus den Sitzungsberichten der Würzburger Physik.-medic. Gesellschaft Würzburg pp. 137-147 (1895).

[6] G. Marconi, Nobel lectures physics 1901-1921 (World Scientific Publishing Co. Pte. Ltd., 1998), chap. Wireless telegraphic communication, pp. 196-222.

[7] W. L. Bragg, Nobel lectures physics 1901-1921 (World Scientific Publishing Co. Pte. Ltd., 1998), chap. The diffraction of X-rays by crystals, pp. 370-382.

[8] R. Kompfner, "The traveling-wave tube as amplifer at microwaves," Proc. IRE 35, 124-124 (1947).

[9] N. Bloembergen, Nobel lectures physics 1981-1990 (World Scientific Publishing Co. Pte. Ltd., 1993), chap. Nonlinear optics and spectroscopy, pp. 12-31.

[10] T. Udem, R. Holzwarth, and T. W. Hänsch, "Optical frequency metrology," Nature 416, 233-237 (2002).

[11] T. Hänsch, Nobel lectures physics 2000-2005 (World Scientific Publishing Co. Ltd., 2008), chap. Passion for precision, pp. 149-173. 
[12] K. Jain, C. G. Willson, and B. J. Lin, "Ultrafast high-resolution contact lithography with excimer lasers," IBM J. Res. Dev. 26, 151-159 (1982).

[13] L. Li, "The advances and characteristics of high-power diode laser materials processing," Opt. Laser. Eng. 34, 231-253 (2000).

[14] C. Bagger and F. O. Olsen, "Review of laser hybrid welding," J. Laser Appl. 17, 2-14 (2005).

[15] M. Tonouchi, "Cutting edge terahertz technology," Nature Photonics 1, 97-105 (2007).

[16] E. Yablonovitch, "Inhibited spontaneous emission in solid-state physics and electronics," Phys. Rev. Lett. 58, 2059-2062 (1987).

[17] S. John, "Strong localization of photons in certain disordered dielectric superlattices," Phys. Rev. Lett. 58, 2486-2489 (1987).

[18] R. Sprik, B. A. van Tiggelen, and A. Lagendijk, "Optical emission in periodic dielectrics," Eur. Phys. Lett. 35, 265-270 (1996).

[19] P. Lodahl, A. F. van Driel, I. S. Nikolaev, A. Irman, K. Overgang, D. Vanmaekelbergh, and W. L. Vos, "Controlling the dynamics of spontaneous emission from quantum dots by photonic crystals," Nature 430, 654-657 (2004).

[20] M. Fujita, S. Takahashi, Y. Tanaka, T. Asano, and S. Noda, "Simultaneous inhibition and redistribution of spontaneous light emission in photonic crystals," Science 308, 1296-1298 (2005).

[21] L. Bechger, P. Lodahl, and W. L. Vos, "Directional fluorescence spectra of laser dye in opal and inverse opal photonic crystals," J. Phys. Chem. B 109, 9980-9988 (2005).

[22] M. Aloshyna, S. Sivakumar, M. Venkataramanan, A. G. Brolo, and F. C. J. M. van Veggel, "Significant suppression of spontaneous emission in $\mathrm{SiO}_{2}$ photonic crystals made with $\mathrm{Tb}^{3+}$-doped $\mathrm{LaF}_{3}$ nanoparticles," J. Phys. Chem. C 111, 4047-4051 (2007).

[23] A. V. Akimov, A. Mukherjee, C. L. Yu, D. E. Chang, A. S. Zibrov, P. R. Hemmer, H. Park, and M. D. Lukin, "Generation of single optical plasmons in metallic nanowires coupled to quantum dots," Nature 450, 402-406 (2007). 
[24] A. Rodenas, G. Zhou, D. Jaque, and M. Gu, "Rare-earth spontaneous emission control in three-dimensional lithium niobate photonic crystals," Adv. Mater. 21, 3526-3530 (2009).

[25] L. Sapienza, H. Thyrrestrup, S. Stobbe, P. D. Garcia, S. Smolka, and P. Lodahl, "Cavity quantum electrodynamics with Anderson-localized modes," Science 327, $1352-1356$ (2010).

[26] W. Cai, A. P. Vasudev, and M. L. Brongersma, "Electrically controlled nonlinear generation of light with plasmonics," Science 333, 1720-1723 (2011).

[27] T. Tumkur, G. Zhu, P. Black, Y. A. Barnakov, and C. E. Bonner, "Control of spontaneous emission in a volume of functionalized hyperbolic metamaterial," Appl. Phys. Lett. 99, 151115 (2011).

[28] M. R. Jorgensen, J. W. Galusha, and M. H. Bart, "Strongly modified spontaneous emission rates in Diamond-structured photonic crystals," Phys. Rev. Lett. 107, 143902 (2011).

[29] M. D. Leistikow, A. P. Mosk, E. Yeganegi, S. R. Huisman, A. Lagendijk, and W. L. Vos, "Inhibited spontaneous emission of quantum dots observed in a 3D photonic band gap," Phys. Rev. Lett. 107, 193903 (2011).

[30] T. Yoshie, O. B. Shchekin, H. Chen, D. G. Deppe, and A. Scherer, "Quantum dot photonic crystal lasers," Electron. Let. 38, 967-968 (2002).

[31] H. Altug, D. Englund, and J. Vučković, "Ultrafast photonic crystal nanocavity laser," Nature Physics 2, 484-488 (2006).

[32] S. Noda, "Photonic crystal lasers - ultimate nanolasers and broad-area coherent lasers," J. Opt. Soc. Am. B 27, B1-B8 (2010).

[33] Y. Gong, B. Ellis, G. Shambat, T. Sarmiento, J. S. Harris, and J. Vučković, "Nanobeam photonic crystal cavity quantum dot laser," Opt. Express 18, 87818789 (2010).

[34] B. Ellis, M. A. Mayer, G. Shambat, T. Sarmiento, J. Harris, E. E. Haller, and J. Vučković, "Ultralow-threshold electrically pumped quantumdot photonic-crystal nanocavity laser," Nature Photonics 5, 297-300 (2011). 
[35] R. Colombelli, K. Srinivasan, M. Troccoli, O. Painter, C. F. Gmachl, D. M. Tennant, A. M. Sergent, D. L. Sivco, A. Y. Cho, and F. Capasso, "Quantum cascade surfaceemitting photonic crystal laser," Science 302, 1374-1377 (2003).

[36] H.-G. Park, S.-H. Kim, S.-H. Kwon, Y.-G. Ju, J.-K. Yang, J.-H. Baek, S.-B. Kim, and Y.-H. Lee, "Electrically driven single-cell photonic crystal laser," Science 305, $1444-1447$ (2004).

[37] K. Nozaki, S. Kita, and T. Baba, "Room temperature continuous wave operation and controlled spontaneous emission in ultrasmall photonic crystal nanolaser," Opt. Express 15, 7506-7504 (2007).

[38] S.-H. Kim, J.-H. Choi, S.-K. Lee, S.-H. Kim, S.-M. Yang, Y.-H. Lee, C. Seassal, P. Regrency, and P. Viktorovitch, "Optofluidic integration of a photonic crystal nanolaser," Opt. Express 16, 6515-6527 (2008).

[39] B. H. Weigl, R. L. Bardell, and C. R. Cabrera, "Lab-on-a-chip for drug development," Adv. Drug Delivery Reviews 55, 349-377 (2003).

[40] M. Loncar, A. Scherer, and Y. Qiu, "Photonic crystal laser sources for chemical detection," Appl. Phys. Lett. 82, 4648-4650 (2003).

[41] C. P. Bacon, Y. Mattley, and R. DeFrece, "Miniature spectroscopic instrumentation: applications to biology and chemistry," Rev. Sci. Instrum. 75, 1-16 (2004).

[42] H. Craighead, "Future lab-on-a-chip technologies for interrogating individual molecules," Nature 442, 387-393 (2006).

[43] P. S. Dittrich and A. Manz, "Lab-on-a-chip: microfluidics in drug discovery," Nat. Rev. Drug. Disc. 5, 210-218 (2006).

[44] J. D. Joannopoulos, S. G. Johnson, J. N. Winn, and R. D. Meade, Photonic crystals: molding the flow of light (Princeton University Press, 2008).

[45] H. Motz, "Is the free-electron laser a laser?" Phys. Lett. 71A, 41-43 (1979).

[46] P. G. O'Shea and H. P. Freund, "Free-electron lasers: status and applications," Science 292, 1853-1858 (2001).

[47] B. W. J. McNeil and N. R. Thompson, "X-ray free-electron lasers," Nature Photonics 4, 814-821 (2010). 
[48] A. Gover and P. Sprangle, "A unified theory of magnetic Bremsstrahlung, electrostatic Bremsstrahlung, Compton-Raman scattering, and Cerenkov-Smith Purcell free-electron lasers," IEEE J. Quantum Electron. QE-17, 1196-1215 (1981).

[49] J. M. J. Madey, "Stimulated emission of Bremsstrahlung in a periodic magnetic field," J. Appl. Phys. 42, 1906-1913 (1971).

[50] R. J. Bakker, C. A. J. van der Geer, D. A. Jaroszynski, A. F. G. van der Meer, D. Oepts, and P. W. van Amersfoort, "Broadband tunability of a far infrared free electron laser," J. Appl. Phys. 74, 1501-1509 (1993).

[51] R. T. Jongma, W. J. van der Zande, A. F. G. van der Meer, U. Lehnert, P. Michel, R. Wünsch, C. A. J. van der Geer, K. Dunkel, C. Piel, and P. J. M. van der Slot, "Design of the Nijmegen high-resolution THz-FEL," in Proc. of FEL 2008 pp. 200203 (2008).

[52] W. Ackermann, G. Asova, V. Ayvazyan, A. Azima, N. Baboi, J. Baehr, V. Balandin, B. Beutner, A. Brandt, A. Bolzmann, R. Brinkmann, O. I. Brovko, M. Castellano, P. Castro, L. Catani, E. Chiadroni, S. Choroba, A. Cianchi, J. T. Costello, D. Cubaynes, J. Dardis, W. Decking, H. Delsim-Hashemi, A. Delserieys, G. Di Pirro, M. Dohlus, S. Duesterer, A. Eckhardt, H. T. Edwards, B. Faatz, J. Feldhaus, K. Floettmann, J. Frisch, L. Froehlich, T. Garvey, U. Gensch, C. Gerth, M. Goerler, N. Golubeva, H.-J. Grabosch, M. Grecki, O. Grimm, K. Hacker, U. Hahn, J. H. Han, K. Honkavaara, T. Hott, M. Huening, Y. Ivanisenko, E. Jaeschke, W. Jalmuzna, T. Jezynski, R. Kammering, V. Katalev, K. Kavanagh, E. T. Kennedy, S. Khodyachykh, K. Klose, V. Kocharyan, M. Koerfer, M. Kollewe, W. Koprek, S. Korepanov, D. Kostin, M. Krassilnikov, G. Kube, M. Kuhlmann, C. L. S. Lewis, L. Lilje, T. Limberg, D. Lipka, F. Loehl, H. Luna, M. Luong, M. Martins, M. Meyer, P. Michelato, V. Miltchev, W. D. Moeller, L. Monaco, W. F. O. Mueller, O. Napieralski, O. Napoly, P. Nicolosi, D. Noelle, T. Nunez, A. Oppelt, C. Pagani, R. Paparella, N. Pchalek, J. Pedregosa-Gutierrez, B. Petersen, B. Petrosyan, G. Petrosyan, L. Petrosyan, J. Pflueger, E. Ploenjes, L. Poletto, K. Pozniak, E. Prat, D. Proch, P. Pucyk, P. Radcliffe, H. Redlin, K. Rehlich, M. Richter, M. Roehrs, J. Roensch, R. Romaniuk, M. Ross, J. Rossbach, V. Rybnikov, M. Sachwitz, E. L. Saldin, W. Sandner, H. Schlarb, B. Schmidt, M. Schmitz, P. Schmueser, J. R. Schneider, E. A. Schneidmiller, S. Schnepp, S. Schreiber, M. Seidel, D. Sertore, A. V. Shabunov, C. Simon, S. Simrock, E. Sombrowski, A. A. Sorokin, P. Spanknebel, R. Spesyvtsev, L. Staykov, B. Steffen, F. Stephan, F. Stulle, H. Thom, K. Tiedtke, 
M. Tischer, S. Toleikis, R. Treusch, D. Trines, I. Tsakov, E. Vogel, T. Weiland, H. Weise, M. Wellhoeffer, M. Wendt, I. Will, A. Winter, K. Wittenburg, W. Wurth, P. Yeates, M. V. Yurkov, I. Zagorodnov, and K. Zapfe, "Operation of a free-electron laser from the extreme ultraviolet to the water window," Nature Photonics 1, 336$342(2007)$.

[53] P. Emma, R. Akre, J. Arthur, R. Bionta, C. Bostedt, J. Bozek, A. Brachmann, P. Bucksbaum, R. Coffee, F.-J. Decker, Y. Ding, D. Dowell, S. Edstrom, A. Fisher, J. Frisch, S. Gilevich, J. Hastings, G. Hays, P. Hering, Z. Huang, R. Iverson, H. Loos, M. Messerschmidt, A. Miahnahri, S. Moeller, H.-D. Nuhn, G. Pile, D. Ratner, J. Rzepiela, D. Schultz, T. Smith, P. Stefan, H. Tompkins, J. Turner, J.Welch, W. White, J. Wu, G. Yocky, and J. Galayda, "First lasing and operation of an Ångstrom-wavelength free-electron laser," Nature Photonics 4, 641-647 (2010).

[54] J. R. Pierce, "Theory of the beam-type traveling-wave tube," Proc. IRE 35, 111-123 (1947).

[55] R. Kompfner and N. T. Williams, "Backward-wave tubes," Proc. IRE 41, 1602-1611 (1953).

[56] H. R. Johnson, "Backward-wave oscillators," Proc. IRE 43, 684-697 (1955).

[57] J. M. Wachtel, "Free-electron lasers using the Smith-Purcell effect," J. Appl. Phys. 50, 49-56 (1979).

[58] K. L. Felch, K. O. Busby, R. W. Layman, D. Kapilow, and J. E. Walsh, "Cerenkov radiation in dielectric-lined waveguides," Appl. Phys. Lett. 38, 601-603 (1981).

[59] E. P. Garate and J. E. Walsh, "The Cerenkov maser at millimeter wavelengths," IEEE Trans. Plasma Sci. PS-13, 524-530 (1985).

[60] E. Jerby, "Traveling-wave free-electron laser," Phys. Rev. A 44, 703-715 (1991).

[61] A. S. Kesar, R. A. Marsh, and R. J. Temkin, "Power measurement of frequencylocked Smith-Purcell radiation," Phys. Rev. ST Accel. Beams 9, 022801 (2006).

[62] T. F. Krauss, "Slow light in photonic crystal waveguides," J. Phys. D: Appl. Phys. 40, 2666-2670 (2007).

[63] J. A. Nation, L. Schächter, F. M. Mako, L. K. Len, W. Peter, C.-M. Tang, and T. Srinivasan-Rao, "Advances in cold cathode physics and technology," Proc. IEEE 87, 865-889 (1999). 
[64] J. H. Booske, "Plasma physics and related challenges of millimeter-wave-to-terahertz and high power microwave generation," Phys. Plasmas 15, 055502 (2008).

[65] J. H. Booske, R. J. Dobbs, C. D. Joye, C. L. Kory, G. R. Neil, G.-S. Park, J. Park, and R. J. Temkin, "Vacuum electronic high power terahertz sources," IEEE Trans. THz Sci. Technol. 1, 54-75 (2011).

[66] L. Brillouin, "A theorem of Larmor and its importance for electrons in magnetic fields," Phys. Rev. 67, 260-266 (1945).

[67] C. Paoloni, M. Mineo, and A. di Carlo, "Vacuum electron tubes for THz applications," in XXXth General Assembly and Scientific Symposium of the International Union of Radio Science pp. 1-4 (2011).

[68] P. J. M. van der Slot, T. Denis, and K.-J. Boller, "The photonic FEL: toward a handheld THz FEL," in Proc. of FEL 2008 pp. 231-234 (2008).

[69] P. J. M. van der Slot, T. Denis, M. W. van Dijk, J. H. H. Lee, and K.-J. Boller, "Photonic free-electron lasers," IEEE Photon. J. 4, 570-573 (2012).

[70] R. Bonifacio, F. Casagrande, and C. Pellegrini, "Hamiltonian model of a freeelectron laser," Opt. Commun. 61, 55-60 (1987).

[71] T. C. Marshall, Free-electron lasers (Macmillan, 1985).

[72] Q. Wang, S. Yu, P. Xun, S. Liu, K. Hu, Y. Chen, and P. Wang, "First operation of a multi-electron-beam Cherenkov free-electron laser oscillator," Appl. Phys. Lett. 59, 2378-2380 (1991).

[73] P. J. M. van der Slot, J. Couperus, W. J. Witteman, A. N. Lebedevc, E. G. Krastelevc, A. V. Agafonov, V. S. Voronin, and V. A. Krasnopolsky, "A Cherenkov free electron laser with high peak power," Nucl. Instrum. Methods Phys. Res. A 358, 100-103 (1995).

[74] J. Wieland, J. Couperus, P. J. M. van der Slot, and W. J. Witteman, "First lasing of a Cherenkov free-electron laser with annular electron beam," Nucl. Instrum. Methods Phys. Res. A 429, 17-20 (1999).

[75] C. Petichakis, R. A. Stuart, C. C. Wright, A. I. Al-ShammaŠa, and J. Lucas, "The dispersion relation for a cylindrical Cherenkov free electron maser," Nucl. Instrum. Methods Phys. Res. A 507, 256-260 (2003). 
[76] V. B. Asgekar and G. Dattoli, "Theory of Cerenkov free electron lasers: An analytical treatment of saturation," Opt. Commun. 255, 309-313 (2005).

[77] H. L. Andrews and C. A. Brau, "Three-dimensional theory of Cerenkov free-electron laser," J. Appl. Phys. 101, 104904 (2007).

[78] I. de la Fuente-Valentin, Theory, design and operation of a compact Cerenkov freeelectron laser, Ph.D. thesis, University of Twente (2007).

http://purl.utwente.nl/publications/57876

[79] E. I. Smirnova, B. E. Carlsten, and L. M. Earley, "Design, fabrication, and lowpower tests of a W-band omniguide traveling-wave tube structure," IEEE Trans. Plasma Sci. 36, 763-767 (2008).

[80] B. D. McVey, M. A. Basten, J. H. Booske, J. Joe, and J. E. Scharer, "Analysis of rectangular waveguide-gratings for amplifier applications," IEEE Trans. Microwave Theory Techn. 42, 995-1003 (1994).

[81] J. Joe, J. Scharer, J. Booske, and B. D. McVey, "Wave dispersion and growth analysis of low-voltage grating Cerenkov amplifiers," Phys. Plasmas 1, 176-188 (1994).

[82] H. L. Andrews and C. A. Brau, "Gain of a Smith-Purcell free-electron laser," Phys. Rev. ST Accel. Beams 7, 070701 (2004).

[83] J. Gardelle and J. T. Donohue, "Three-dimensional simulations of coherent SmithPurcell radiation using a particle-in-cell code," IEEE Trans. Electron Devices 56, 769-774 (2009).

[84] D. Li, M. Hangyo, Y. Tsunawaki, Z. Yang, Y. Wei, S. Miyamoto, M. R. Asakawa, and K. Imasaki, "Growth rate and start current in Smith-Purcell free-electron lasers," Appl. Phys. Lett. 100, 191101 (2012).

[85] J. Gardelle, P. Modin, and J. T. Donohue, "Observation of copious emission at the fundamental frequency by a Smith-Purcell free-electron laser with sidewalls," Appl. Phys. Lett. 100, 131103 (2012).

[86] H. Heffner, "Analysis of the backward-wave traveling-wave tube," Proc. IRE 42, 930-937 (1954).

[87] R. G. E. Hutter, Beam and wave electronics in microwave tubes (Boston Technical Pub., 1965). 
[88] A. H. W. Beck, Space-charge waves (Pergamon Press Ltd., 1958).

[89] A. S. Gilmour, Jr., Principles of traveling wave tubes (Artech, 1994).

[90] C. L. Kory, "Investigation of fully three-dimensional helical RF field effects on TWT beam/circuit interaction," IEEE Trans. Electron Devices 48, 1718-1726 (2001).

[91] D. Chernin, T. M. Antonsen, B. Levush, and D. Whaley, "A three-dimensional multifrequency large signal model for helix traveling wave tubes," IEEE Trans. Electron Devices 48, 3-11 (2001).

[92] S.-S. Jung, A. V. Soukhov, and G.-S. Park, "Large-signal simulations of the interaction between an electron beam and a traveling electromagnetic wave in a helical structure," J. Korean Phys. Soc. 39, 1087-1094 (2001).

[93] S. Bhattacharjee, J. H. Booske, C. L. Kory, D. W. van der Weide, S. Limbach, S. Gallagher, J. D. Welter, M. R. Lopez, R. M. Gilgenbach, R. L. Ives, M. E. Read, R. Divan, and D. C. Mancini, "Folded waveguide traveling-wave tube sources for terahertz radiation," IEEE Trans. Plasma Sci. 32, 1032 (2004).

[94] P. Palluel and A. K. Goldberger, "The O-type carcinotron tube," Proc. IRE 44, 333-345 (1956).

[95] Y. Carmel, J. Ivers, R. E. Kribel, and J. Nation, "Intense coherent Cherenkov radiation due to the interaction of a relativistic electron beam with a slow-wave structure," Phys. Rev. Lett. 33, 1278-1282 (1974).

[96] J. A. Dayton, V. O. Heinen, N. Stankiewicz, and T. M. Wallett, "Submillimeter backward wave oscillator," Int. J. Infrared. Milli. Waves 8, 1257-1268 (1987).

[97] D. K. Abe, Y. Carmel, S. M. Miller, A. Bromborsky, B. Levush, T. M. Antonsen, and W. W. Destler, "Experimental studies of overmoded relativistic backward-wave oscillators," IEEE Trans. Plasma Sci. 26, 591-604 (1998).

[98] J. Zhang, H.-H. Zhong, and L. Luo, "A novel overmoded slow-wave high-power microwave (HPM) generator," IEEE Trans. Plasma Sci. 32, 2236-2242 (2004).

[99] D. H. Kim, H. C. Jung, Y. B. Kang, and G. S. Park, "Numerical study on a rippledwall slow-wave structure for a backward wave oscillator," J. Korean Phys. Soc. 44, 1298-1302 (2004). 
[100] M. Mineo and C. Paoloni, "Corrugated rectangular waveguide tunable backwardwave oscillator for terahertz applications," IEEE Trans. Electron Devices 57, 1481$1484(2010)$.

[101] X. Ge, H. Zhong, B. Qian, J. Zhang, L. Liu, L. Gao, C. Yuan, and J. He, "Asymmetric-mode competition in a relativistic backward wave oscillator with a coaxial slow-wave structure," Appl. Phys. Lett. 97, 241501 (2010).

[102] F. S. Rusin and G. D. Bogomolov, "The orotron, an electronic device with an open resonator and a reflecting grating," Radiophys. and Quantum Electronics 11, 430433 (1968).

[103] A. Gover and Z. Livni, "Operation regimes of Cerenkov-Smith-Purcell free-electron lasers and T.W. amplifiers," Opt. Commun. 26, 375-380 (1978).

[104] G. Toraldo di Francia, "On the theory of some Cerenkovian effects," Il Nuovo Cimento 16, 61-77 (1960).

[105] S. H. Strogatz, Nonlinear dynamics and chaos (Perseus Books Group, 1994).

[106] H. P. Freund, "A re-examination of the traveling-wave interaction," IEEE Trans. Plasma Sci. 22, 499-503 (1994).

[107] V. Kumar and K.-J. Kim, "Analysis of Smith-Purcell free-electron lasers," Phys. Rev. E 73, 026501 (2006).

[108] J. M. Dawson, "Particle simulation of plasmas," Rev. Mod. Phys. 55, 403-447 (1983).

[109] C. K. Birdsall and A. B. Langdon, Plasma physics via computer simulation (Adam Hilger Book Company, 1991).

[110] D. Tskhakaya, K. Matyash, R. Schneider, and F. Taccogna, "The particle-in-cell method," Contrib. Plasma Phys. 47, 563-594 (2007).

[111] P. A. Cerenkov, "Visible radiation produced by electrons moving in a medium with velocities exceeding that of light," Phys. Rev. 52, 378-379 (1937).

[112] I. Frank and I. Tamm, "Coherent visible radiation of fast electrons passing through matter," Dokl. Aka. Nauk. SSSR 14, 109-114 (1937).

[113] J. V. Jelly, Cerenkov radiation and its applications (Pergammon, 1958). 
[114] A. Ascoli-Balzanelli and R. Ascoli, "The Cerenkov effect produced by single particles in gases," Il Nuovo Cimento 6, 1392-1408 (1957).

[115] J. G. Linhardt, "Cerenkov radiation of electrons moving parallel to a dielectric boundary," J. Appl. Phys. 26, 527-533 (1955).

[116] M. Danos, "Cerenkov radiation from extended electron beams," J. Appl. Phys. 26, 62-68 (1955).

[117] S. J. Smith and E. M. Purcell, "Visible light from localized surface charges moving across a grating," Phys. Rev. 92, 1069-1069 (1953).

[118] G. Adamo, K. F. MacDonald, Y. H. Fu, C.-M. Wang, D. P. Tsai, F. J. García de Abajo, and N. I. Zheludev, "Light well: A tunable free-electron light source on a chip," Phys. Rev. Lett. 103, 113901 (2009).

[119] C. Luo, M. Ibanescu, S. G. Johnson, and J. D. Joannopoulos, "Cerenkov radiation in photonic crystals," Science 299, 368-371 (2003).

[120] V. L. Ginzburg, "Transition radiation and transition scattering," Phys. Scripta T2/1, 182-191 (1982).

[121] S. G. Johnson and J. D. Joannopoulos, "Block-iterative frequency-domain methods for Maxwell's equations in a planewave basis," Opt. Express 8, 173-190 (2001).

[122] C. Kittel, Introduction to solid state physics (John Wiley \& Sons, 2004).

[123] N. W. Ashcroft and N. D. Mermin, Solid state physics (Holt, Rinehard \& Winston, 1976).

[124] R. E. Collin, Field theory of guided waves (Wiley-IEEE-Interscience, 1991).

[125] C. Kremers, D. N. Chigrin, and J. Kroha, "Theory of Cherenkov radiation in periodic dielectric media: emission spectrum," Phys. Rev. A 79, 013829 (2009).

[126] S. Ramo, "Space charge and field waves in an electron beam," Phys. Rev. 56, 276283 (1939).

[127] C. C. Johnson, Field and wave electrodynamics (McGraw-Hill, 1965).

[128] L. Schächter, Beam-wave interaction in periodic and quasie-periodic structures (Springer, 2011). 
[129] M. V. Kuzelev, "Quantum theory of stimulated Cerenkov radiation of transverse electromagnetic waves by a low-density electron beam in a medium," Quantum Electron. 40, 83-87 (2010).

[130] B. M. Cowan, "Two-dimensional photonic crystal accelerator structures," Phys. Rev. ST Accel. Beams 6, 101301 (2003).

[131] B. M. Cowan, "Three-dimensional dielectric photonic crystal structures for laserdriven acceleration," Phys. Rev. ST Accel. Beams 11, 011301 (2008).

[132] C. McGuinness, E. Colby, and R. L. Byer, "Accelerating electrons with lasers and photonic crystals," J. Mod. Optic. 56, 2142-2147 (2009).

[133] R. A. Marsh, M. A. Shapiro, R. J. Temkin, V. A. Dolgashev, L. L. Laurent, J. R. Lewandowski, A. D. Yeremian, and S. G. Tantawi, "X-band photonic band-gap accelerator structure breakdown experiment," Phys. Rev. ST Accel. Beams 14, 021301 (2011).

[134] J. A. Swegle, J. W. Poukey, and G. T. Leifeste, "Backward-wave oscillators with rippled wall resonators: analyticaltheory and numerical simulations," Phys. Fluids 28, $2882(1985)$.

[135] Y. Zhang, Y. L. Mo, R. M. Xu, B. Yan, and X. Q. Xie, "An investigation of periodic waveguides with axial and azimuthal corrugations," IEEE Trans. Plasma Sci. 33, 2017-2026 (2005).

[136] A. N. Vlasov, G. S. Nusinovich, and B. Levush, "Effect of the zero spatial harmonic in a slow electromagnetic wave on operation of relativistic backward-wave oscillators," Phys. Plasmas 4, 1402-1412 (1997).

[137] O. Buneman, "Dissipation of currents in ionized media," Phys. Rev. 115, 503-517 (1959).

[138] B. Goplen, L. Ludeking, D. Smithe, and G. Warren, "User-configurable MAGIC for electromagnetic PIC calculations," Comput. Phys. Commun. 87, 54-86 (1995).

[139] M. Botton, T. M. Antonsen, B. Levush, K. T. Nguyen, and A. N. Vlasov, "MAGY: a time-dependent code for simulation of slow and fast microwave sources," IEEE Trans. Plasma Sci. 26, 882-892 (1998). 
[140] J. Forest, A. Hilgers, B. Thiebault, L. Eliasson, J.-J. Berthelier, and H. de Feraudy, "An open-source spacecraft plasma interaction simulation code PicUp3D: tests and validations," IEEE Trans. Plasma Sci. 34, 2103-2113 (2006).

[141] CST AG, CST Microwave Studio 2011, Bad Nauheimer Str. 19, 64289 Darmstadt, Germany.

www.cst.de

[142] G. Lapenta, "Particle simulations of space weather," J. Comput. Phys. 231, 795-821 (2012).

[143] X. Davoine, E. Lefebvre, J. Faure, C. Rechatin, and A. Lifschitz, "Simulation of quasimonoenergetic electron beams produced by colliding pulse wakefield acceleration," Phys. Plasmas 15, 113102 (2008).

[144] T. J. T. Kwan, "Application of particle-in-cell simulation in free-electron lasers," IEEE J. Quantum Electron. QE-17, 1394-1408 (1981).

[145] A. Hirata and T. Shiozawa, "Three-dimensional analysis of a Cherenkov laser via particle simulation," IEEE J. Quantum Electron. 34, 1802-1806 (1998).

[146] X. Ge, H. Zhong, B. Qian, L. Liu, Y. Liu, L. Li, T. Shu, and J. Zhang, "Transversal and longitudinal mode selections in double-corrugation coaxial slow-wave devices," Phys. Plasmas 16, 063107 (2009).

[147] H. Okuda, "Nonphysical noises and instabilities in plasma simulation due to a spatial grid," J. Comput. Phys. 10, 475-486 (1972).

[148] M. J. de Loos, S. B. van der Geer, C. A. J. van der Geer, A. G. A. Verhoeven, and W. H. Urbanus, "The general particle tracer code applied to the fusion free-electron maser," Nucl. Instrum. Methods Phys. Res. B 139, 481-486 (1998).

[149] R. L. Burden, J. D. Faires, and A. C. Reynolds, Numerical analysis (Weber \& Schmidt, 1981).

[150] M. Amini, J. W. Eastwood, and R. W. Hockney, "Time integration in particle models," Comput. Phys. Commun. 44, 83-93 (1987).

[151] J. Kästel and M. Fleischhauer, "Suppression of spontaneous emission and superradiance over macroscopic distances in media with negative refraction," Phys. Rev. A 71, 011804 (2005). 
[152] V. Yannopapas, E. Paspalakis, and N. V. Vitanov, "Plasmon-induced enhancement of quantum interference near metallic nanostructures," Phys. Rev. Lett. 103, $063602(2009)$.

[153] D. A. B. Miller, "Device requirements for optical interconnects to silicon chips," Proc. IEEE 97, 1166-1185 (2009).

[154] C. Cohen-Tannoudji, B. Diu, and F. Laloe, Quantum mechanics (2 vol. set) (Wiley, 1992).

[155] K. Yamamoto, R. Sakakibara, S. Yano, Y. Segawa, Y. Shibata, K. Ishi, T. Ohsaka, T. Hara, Y. Kondo, H. Miyazaki, F. Hinode, T. Matsuyama, S. Yamaguti, and K. Ohtaka, "Observation of millimeter-wave radiation generated by the interaction between an electron beam and a photonic crystal," Phys. Rev. E 69, 045601 (2004).

[156] C. Kremers and D. N. Chigrin, "Spatial distribution of Cherenkov radiation in periodic dielectric media,” J. Opt. A: Pure Appl. Opt. 11, 114008 (2009).

[157] G. Adamo, K. F. MacDonald, Y. H. Fu, D. P. Tsai, F. J. García de Abajo, and N. I. Zheludev, "Tuneable electron-beam-driven nanoscale light source," J. Opt. 12, $024012(2010)$.

[158] F. J. García de Abajo, "Optical excitations in electron microscopy," Rev. Mod. Phys. 82, 209-275 (2010).

[159] K. Batrakov and S. Sytova, "Mathematical modelling of multiwave volume freeelectron laser: basic principles and numerical experiments," Math. Model. Anal. 11, 13-22 (2006).

[160] S. M. Miller, T. M. Antonsen, B. Levush, A. Bromborsky, and D. K. Abe, "Theory of relativistic backward wave oscillators operating near cutoff," Phys. Plasmas 1, 730-740 (1994).

[161] M. Mineo and C. Paoloni, "Double-corrugated rectangular waveguide slow-wave structure for terahertz vacuum devices," IEEE Trans. Electron Devices 57, 31693175 (2010).

[162] W. Schottky, "Über spontane Stromschwankungen in verschiedenen Elektrizitätsleitern," Annalen der Physik 57, 541-567 (1918).

[163] A. J. Rack, "Effect of space charge and transit time on the shot noise in diodes," Bell System Tech. J. 17, 592-619 (1938). 
[164] C. C. Cutler, "The nature of power saturation in traveling wave tubes," Bell Sys. Tech. J. 35, 841-867 (1956).

[165] T. K. Ishii, Handbook of microwave technology: components and devices (Academic Press, 1995).

[166] M. W. van Dijk, The photonic free-electron laser: a numerical study of its fundamental physics, Master's thesis, University of Twente (2012).

http://purl.utwente.nl/essays/62124

[167] J. D. Jackson, Classical electrodynamics (John Wiley \& Sons, Inc., 1998).

[168] S. Humphries, Charged particle beams (Wiley-Interscience, 1990).

[169] A. E. Siegman, Lasers (University Science Books, 1986).

[170] E. Jerby, "Linear analysis of periodic-waveguide cyclotron maser interaction," Phys. Rev. E 49, 4487-4496 (1994).

[171] B. Levush, T. M. Antonsen, A. B. W.-R. Lou, and Y. Carmel, "Theory of relativistic backward-wave oscillators with end reflections," IEEE Trans. Plasma Sci. 20, 263280 (1992).

[172] S. H. Gold and G. S. Nusinovich, "Review of high-power microwave source research," Rev. Sci. Instrum. 68, 3945-3974 (1997).

[173] E. Naesset, "Predicting forest stand characteristics with airborne scanning laser using a practical two-stage procedure and field data," Remote Sens. Environ. 80, 88-99 (2002).

[174] J. B. Campbell, Introduction to remote sensing (The Guilford Press, 2006).

[175] M. Govender, K. Chetty, and H. Bulcock, "A review of hyperspectral remote sensing and its application in vegetation and water resource studies," Water SA 33, 145-152 (2007).

[176] P. D. D. V. Yarlagadda and T. C. Chai, "An investigation into welding of engineering thermoplastics using focused microwave energy," J. Mater. Process. Tech. 74, 199212 (1998).

[177] K. E. Haque, "Microwave energy for mineral treatment processes-a brief review," Int. J. Miner. Process. 57, 1-24 (1999). 
[178] R. J. Wise and I. D. Froment, "Microwave welding of thermoplastics," J. Mater. Sci. 36, 5935-5954 (2001).

[179] D. A. Jones, T. P. Lelyveld, S. D. Mavrofidis, S. W. Kingman, and N. J. Miles, "Microwave heating applications in environmental engineering-a review," Resources, Conservation and Recycling 34, 75-90 (2002).

[180] A. Suzuki and M. Ishihara, "Application of $\mathrm{CO}_{2}$ laser heating zone drawing and zone annealing to nylon 6 fibers," J. Appl. Polym. Sci. 83, 1711-1716 (2002).

[181] E. J. Minay, A. R. Boccaccini, P. Veronesi, V. Cannillo, and C. Leonelli, "Processing of novel glass matrix composites by microwave heating," J. Mater. Process. Tech. 155-156, 1749-1755 (2004).

[182] R. Schaeffer, Fundamentals of laser micromachining (Taylor \& Francis, 2012).

[183] A. W. Guy, "History of biological effects and medical applications of microwave energy," IEEE Trans. Microwave Theory Techn. 32, 1182-1200 (1984).

[184] C. Sturesson, Medical laser-induced thermotherapy-models and applications, Ph.D. thesis, Lund Institute of Technology (1998). http://www.lu.se/lup/publication/38965

[185] D. I. Thwaites and J. B. Tuohy, "Back to the future: the history and development of the clinical linear accelerator," Phys. Med. Biol. 51, R343-62 (2006).

[186] S. M. Hanna, "Role of microwave accelerators in cancer treatment," in IEEE/MTTS International Microwave Symposium pp. 1337-1340 (2007).

[187] N. S. Xu and S. E. Huq, "Novel cold cathode materials and applications," Mater. Sci. Eng. R Rep. 48, 47-189 (2005).

[188] R. L. Ives, G. Collins, M. Read, G. Miram, and D. Marsden, "Electron guns for terahertz vacuum electron sources," IEEE Trans. THz Sci. Technol. 4, 230-239 (2011).

[189] S. Sytova, "Numerical analysis of lasing dynamics in volume free electron laser," Math. Model. Anal. 13, 263-274 (2008).

[190] R. M. Phillips and D. W. Sprehn, "High-power klystrons for the next linear collider," Proc. IEEE 87, 738-751 (1999). 
[191] M. Thumm, "High power gyro-devices for plasma heating and other applications," Int. J. Infrared. Milli. Waves 26, 483-503 (2005).

[192] Z.-H. Li and Y. Qi, "Mode control in an oversized backward wave oscillator," Phys. Plasmas 15, 093104 (2008).

[193] P. W. Smith, "Mode selection in lasers," Proc. IEEE 60, 422-440 (1972).

[194] D. G. Hall, R. J. Smith, and R. R. Rice, "Pump-size effects in Nd:YAG lasers," Appl. Opt. 19, 3041-3043 (1980).

[195] T. Denis, S. Hahn, S. Mebben, R. Wilhelm, C. Kolleck, J. Neumann, and D. Kracht, "Compact diode stack end pumped Nd:YAG amplifier using core doped ceramics," Appl. Opt. 49, 811-816 (2010).

[196] W. Koechner, Solid-state laser engineering (Springer, 2006).

[197] G. Dattoli, T. Letardi, A. Renieri, and J. M. J. Madey, "Lawson-Penner limit and single passage free electron lasers performances," IEEE J. Quantum Electron. 20, 637-646 (1984).

[198] M. I. Petelin, "Mode selection in high power microwave sources," IEEE Trans. Electron Devices 48, 129-133 (2001).

[199] V. M. Baev, K.-J. Boller, A. Weiler, and P. E. Toschek, "Detection of spectrally narrow light emission by laser intra-cavity spectroscopy," Opt. Commun. 62, 380384 (1987).

[200] G. S. Nusinovich, S. J. Cooke, M. Botton, and B. Levush, "Wave coupling in sheetand multiple-beam traveling-wave tubes," Phys. Plasmas 16, 063102 (2009).

[201] C. Paoloni, Di Carlo, Aldo, F. Brunetti, M. Mineo, G. Ulisse, A. Durand, V. Krozer, M. Kotiranta, A. M. Fiorello, M. Dispenza, A. Secchi, V. Zhurbenko, F. Bouamrane, T. Bouvet, S. Megtert, and E. Tamburri, "Design and fabrication of a $1 \mathrm{THz}$ backward wave amplifier," IEEE Trans. THz Sci. Technol. 4, 149-163 (2011).

[202] A. S. Pobedonostsev, E. A. Gelvich, M. I. Lopin, A. M. Alexeyenko, A. A. Negirev, and B. V. Sazonov, "Multiple-beam microwave tubes," in IEEE/MTT-S International Microwave Symposium pp. 1131-1134 (1993).

[203] J. C. Tucek, M. A. Basten, D. A. Gallagher, K. E. Kreischer, R. Lai, V. Radisic, K. Leong, and R. Mihailovich, "A $100 \mathrm{~mW}, 0.670$ THz Power Module," in IEEE international vacuum electronics conference (IVEC) (2012). 
[204] H. K. Park, J. R. Oh, and Y. R. Do, "2D SiNx photonic crystal coated $\mathrm{Y}_{3} \mathrm{Al}_{5} \mathrm{O}_{12}: \mathrm{Ce}^{3+}$ ceramic plate phosphor for high-power white light-emitting diodes," Opt. Express 19, 25 593-25601 (2011).

[205] M. Florescu, H. Lee, I. Puscasu, M. Pralle, L. Florescu, D. Z. Ting, and J. P. Dowling, "Improving solar cell efficiency using photonic band-gap materials," Sol. energ. mat. sol. c. 91, 1599-1610 (2007).

[206] D.-H. Ko, J. R. Tumbleston, L. Zhang, S. Williams, J. M. DeSimone, R. Lopez, and E. T. Samulski, "Photonic crystal geometry for organic solar cells," Nano Letters 9, 2742-2746 (2009).

[207] A. F. Oskooi, D. Roundy, M. Ibanescu, P. Bermel, J. D. Joannopoulos, and S. G. Johnson, "MEEP: A flexible free-software package for electromagnetic simulations by the FDTD method," Comput. Phys. Commun. 181, 687-702 (2010).

[208] Vector Fields Software, Concerto V7.5, Cobham CTS Ltd., Brook Road, BH21 2BJ, Wimborne, Dorset, United Kingdom. http://www.cobham.com

[209] N. W. Ashcroft and N. D. Mermin, Solid state physics (Holt, Rinehard \& Winston, 1976), chap. 30, pp. 616-Ü620.

[210] A. F. Koenderink and W. L. Vos, "Optical properties of real photonic crystals: anomalous diffuse transmission," J. Opt. Soc. Am. B 22, 1075-1084 (2005).

[211] D. Englund and J. Vučković, "A direct analysis of photonic nanostructures," Opt. Express 14, 3472-3483 (2006).

[212] U. K. Khankhoje, S.-H. Kim, B. C. Richards, J. Hendrickson, J. Sweet, J. D. Olitzky, G. Khitrova, H. M. Gibbs, and A. Scherer, "Modelling and fabrication of GaAs photonic-crystal cavities for cavity quantum electrodynamics," Nanotechnology 21, $065202(2010)$.

[213] B. Hecht, H. Bielefeldt, Y. Inouye, D. W. Pohl, and L. Novotny, "Facts and artifacts in near-field optical microscopy," J. Appl. Phys. 81, 2492-2498 (1997).

[214] R. Carminati, A. Madrazo, M. Nieto-Vesperinas, and J.-J. Greffet, "Optical content and resolution of near-field optical images: influence of the operating mode," J. Appl. Phys. 82, 501-509 (1997). 
[215] K. D. Weston and S. K. Buratto, "A reflection near-field scanning optical microscope technique for subwavelength resolution imaging of thin organic films," J. Phys. Chem. B 101, 5684-5691 (1997).

[216] P. J. Valle, J.-J. Greffet, and R. Carminati, "Optical contrast, topographic contrast and artifacts in illumination-mode scanning near-field optical microscopy," J. Appl. Phys. 86, 648-656 (1999).

[217] M. Labardi, S. Patane, and M. Allegrini, "Artifact-free near-field optical imaging by apertureless microscopy," Appl. Phys. Lett. 77, 621-623 (2000).

[218] M. L. M. Balistreri, H. Gersen, J. P. Korterik, L. Kuipers, and N. F. van Hulst, "Tracking femtosecond laser pulses in space and time," Science 294, 1080-1082 (2001).

[219] S. I. Bozhevolnyi, V. S. Volkov, J. Arentoft, A. Boltasseva, T. Sondergaard, and M. Kristensen, "Direct mapping of light propagation in photonic crystal waveguides," Opt. Commun. 212, 51-55 (2002).

[220] L. Okamoto, M. Loncar, T. Yoshie, A. Scherer, Y. Qiu, and P. Gogna, "Near-field scanning optical microscopy of photonic crystal nanocavities," Appl. Phys. Lett. 82, 1676-1678 (2003).

[221] E. Flück, N. F. van Hulst, W. L. Vos, and L. Kuipers, "Near-field optical investigation of three-dimensional photonic crystals," Phys. Rev. E 68, 15601(R) (2003).

[222] P. Kramper, M. Agio, C. M. Soukoulis, A. Birner, F. Müller, R. B. Wehrspohn, U. Gösele, and V. Sandoghdar, "Highly directional emission from photonic crystal waveguides of subwavelength width," Phys. Rev. Lett. 92, 113903 (2004).

[223] H.-H. Tao, R.-J. Liu, Z.-Y. Li, S. Feng, Y.-Z. Liu, C. Ren, B.-Y. Cheng, D.-Z. Zhang, H.-Q. Ma, L.-A. Wu, and Z.-B. Zhang, "Mapping of complex optical field patterns in multimode photonic crystal waveguides by near-field scanning optical microscopy," Phys. Rev. B 74, 205111 (2006).

[224] M. Abashin, P. Tortora, I. Märki, U. Levy, W. Nakagawa, L. Vaccaro, H. Herzig, and Y. Fainman, "Near-field characterization of propagating optical modes in photonic crystal waveguides," Opt. Express 14, 1643-1657 (2006).

[225] K. G. Lee, H. W. Kihm, J. E. Kihm, W. J. Choi, H. Kim, C. Ropers, D. J. Park, Y. C. Yoon, S. B. Choi, D. H. Woo, J. Kim, B. Lee, Q. H. Park, C. Lienau, and 
D. S. Kim, "Vector field microscopic imaging of light," Nature Photonics 1, 53-56 (2007).

[226] M. A. Seo, A. J. L. Adam, J. H. Kang, J. W. Lee, S. C. Jeoung, Q. H. Park, P. C. M. Planken, and D. S. Kim, "Fourier-transform terahertz near-field imaging of one-dimensional slit arrays: mapping of electric-field-, magnetic-field-, and Poynting vectors," Opt. Express 15, 11 781-11789 (2007).

[227] S. Vignolini, F. Intonti, F. Riboli, D. S. Wiersma, L. Balet, L. H. Li, M. Francardi, A. Gerardino, A. Fiore, and M. Gurioli, "Polarization-sensitive near-field investigation of photonic crystal microcavities," Appl. Phys. Lett. 94, 163102 (2009).

[228] M. Schnell, A. Garcia-Etxarri, J. Alkorta, J. Aizpurua, and R. Hillenbrand, "Phaseresolved mapping of the near-field vector and polarization state in nanoscale antenna gaps," Nano Letters 10, 3524-3528 (2010).

[229] J. Dahdah, M. Pilar-Bernal, N. Courjal, G. Ulliac, and F. Baida, "Near-field observations of light confinement in a two dimensional lithium niobate photonic crystal cavity," J. Appl. Phys 110, 074318 (2011).

[230] D. C. Kohlgraf-Owens, S. Sukhov, and A. Dogariu, "Optical-force-induced artifacts in scanning probe microscopy," Opt. Lett. 36, 4758-4760 (2011).

[231] M. Esslinger, J. Dorfmüller, W. Khunsin, R. Vogelgesang, and K. Kern, "Background-free imaging of plasmonic structures with cross-polarized apertureless scanning near-field optical microscopy," Rev. Sci. Instrum. 83, 033704 (2012).

[232] Y. Lee, X. Lu, Y. Hao, S. Yang, R. Ubic, J. R. G. Evans, and C. G. Parini, "Rapid prototyping of ceramic millimeterwave metamaterials: simulations and experiments," Microwave and optical technologies letters 49, 2090-2093 (2007).

[233] P. Pengvanich, D. Chernin, Y. Y. Lau, J. W. Luginsland, and R. M. Gilgenbach, "Effect of random circuit fabrication errors on small-signal gain and phase in travelingwave tubes," IEEE Trans. Electron Devices 55, 916-924 (2008).

[234] D. Chernin, I. Rittersdorf, Y. Y. Lau, T. M. Antonsen, and B. Levush, "Effects of multiple internal reflections on the small-signal gain and phase of a TWT," IEEE Trans. Electron Devices 59, 1542-1550 (2012).

[235] R. O. Jenkins, "A review of thermionic cathodes," Vacuum 19, 353-359 (1969).

[236] J. L. Cronin, "Modern dispenser cathodes," Proc. IEEE 128, 19-32 (1981). 
[237] L. Falce, "Dispenser cathodes: the current state of the technology," IEDM Techn. Digest 29, 448 (1983).

[238] L. R. Falce, "High current density dispenser cathode surface activation phenomena in long life vacuum electron devices," in 29th IEEE International Conference on Plasma Science (ICOPS) p. 128 (2002).

[239] M. Clark and C. Gilmour, personal communication, TMD Technologies Limited, Swallowfield Way, UB3 1DQ, Hayes, Middlesex, United Kingdom.

[240] L. C. Maier, Field strength measurements in resonant cavities, Ph.D. thesis, Massachusetts Institute of Technology (1949).

[241] L. C. Maier and J. C. Slater, "Field strength measurements in resonant cavities," J. Appl. Phys. 23, 68-78 (1952).

[242] B. Guru and H. Hiziroglu, Electromagnetc field theory and fundamentals (Pws Pub Co, 1997).

[243] H. Guo, Y. Carmel, W. R. Lou, L. Chen, J. Rodgers, D. K. Abe, A. Bromborsky, W. Destler, and V. Granatstein, "A novel highly accurate synthetic technique for determination of the dispersive characteristics in periodic slow wave circuits," IEEE Trans. Microwave Theory Techn. 40, 2086-2094 (1992).

[244] M. Kageshima, H. Jensenius, M. Dienwiebel, Y. Nakayama, H. Tokumoto, S. P. Jarvis, , and T. H. Oosterkamp, "Noncontact atomic force microscopy in liquid environment with quartz tuning fork and carbon nanotube probe," Appl. Surf. Sci. 188, 440-444 (2002).

[245] M. Frimmer, Y. Chen, and A. F. Koenderink, "Scanning emitter lifetime imaging microscopy for spontaneous emission control," Phys. Rev. Lett. 107, 123602 (2011).

[246] V. G. Baryshevsky, N. A. Belous, A. A. Gurinovich, V. A. Evdokimov, P. V. Molchanov, A. V. Oskin, and P. F. Safronov, "Experimental study of volume free electron laser using a "grid" photonic crystal with variable period," in Proc. of FEL 2007 (2007).

[247] V. G. Baryshevsky, N. A. Belous, A. A. Gurinovich, E. A. Gurnevich, V. A. Evdokimov, and P. V. Molchanov, "Experimental studies of volume FELs with a photonic crystal made of foils," in Proc. of FEL 2010 (2010). 
[248] K. T. Nguyen, D. E. Pershing, D. K. Abe, B. Levush, F. N. Wood, J. P. Calame, J. A. Pasour, J. J. Petillo, M. Cusick, M. J. Cattelino, and E. L. Wright, "Electron gun design for fundamental mode S-band multiple-beam amplifiers," IEEE Trans. Plasma Sci. 32, 1212-1222 (2004).

[249] K. T. Nguyen, J. A. Pasour, T. M. Antonsen, P. B. Larsen, J. J. Petillo, and B. Levush, "Intense sheet electron beam transport in a uniform solenoidal magnetic field," IEEE Trans. Electron Devices 56, 744-752 (2009).

[250] J. H. H. Lee, T. Denis, P. J. M. van der Slot, and K.-J. Boller, "The design of a multi-beam electron gun for a photonic free-electron laser," in Proc. of FEL 2011 pp. $427-430$ (2011).

[251] TMD Technologies Limited, Electron gun specifications, Swallowfield Way, UB3 1DQ, Hayes, Middlesex, United Kingdom.

http://www.tmd.co.uk

[252] J. R. Pierce, Theory and design of electron beams (Van Nostrand, 1954).

[253] R. True, "A theory for coupling gridded gun design with PPM focussing," IEEE Trans. Electron Devices 31, 353-362 (1984).

[254] D. A. Watkins and N. Rynn, "Effect of velocity distribution on traveling-wave tube gain," J. Appl. Phys. 25, 1375-1379 (1954).

[255] J. R. Hechtel, "Magnetic focusing of electron beams in the presence of transverse velocity components," IEEE Trans. Electron Devices ED-28, 473-482 (1981).

[256] Vector Fields Software, Opera V13.0, Cobham CTS Ltd., Brook Road, BH21 2BJ, Wimborne, Dorset, United Kingdom.

http://www.cobham.com

[257] D. M. Pozar, Microwave engineering (Wiley, 2012).

[258] J. H. Osmundsen and N. Gade, "Influence of optical feedback on laser frequency spectrum and threshold conditions," IEEE J. Quantum Electron. QE-19, 465-469 (1983).

[259] H. Kogelnik and T. Li, "Laser beams and resonators," Appl. Opt. 5, 1550-1567 (1966).

[260] E. Hecht, Optics (Addison Wesley, 2002). 
[261] S. Olivier, H. Benisty, C. Weisbuch, C. Smith, T. Krauss, and R. Houdre, "Coupledmode theory and propagation losses in photonic crystal waveguides," Opt. Express 11, 1490-1496 (2003).

[262] E. Istrate, A. A. Green, and E. H. Sargent, "Behavior of light at photonic crystal interfaces," Phys. Rev. B 71, 195122 (2005).

[263] B. Momeni, M. Badieirostami, and A. Adibi, "Accurate and efficient techniques for the analysis of reflection at the interfaces of three-dimensional photonic crystals," J. Opt. Soc. Am. B 24, 2957-2963 (2007). 



\section{Samenvatting}

Coherente elektromagnetische golven worden veel gebruikt in verschillende onderzoeksvelden en hebben vele toepassingen. Vrijwel elk deel van het elektromagnetische spectrum, variërend van radiogolven tot harde Röntgenstraling, wordt succesvol gebruikt ten behoeve van de mensheid. Daarom is het ook niet verassend dat, ondanks de grote verscheidenheid aan bestaande bronnen waarmee elektromagnetische golven opgewekt worden, er een aanhoudende vraag is naar nieuwe bronnen met verbeterde eigenschappen aangepast aan specifieke behoeftes.

Een belangrijke recente ontwikkeling in het continue streven naar nieuwe bronnen, is het gebruik van materialen met een periodieke structuur op de schaal van de elektromagnetische golflengte. Deze zogenoemde fotonische kristallen beïnvloeden op een fundamentele manier de generatie van licht. Deze vorm van controle stelt wetenschappers in staat lasers met unieke eigenschappen te ontwikkelen. Voorbeelden zijn ultrasnel gemoduleerde lasers of lasers met een extreem lage drempel. Doordat de karakteristieke lengte van fotonische kristallen van de orde van de golflengte is, zijn deze lasers zeer compact te maken. Het is bijvoorbeeld goed mogelijk dat op fotonische kristallen gebaseerde lasers diodelasers aanvullen in lab-on-a-chip toepassingen voor eenvoudige en snelle analyse van biologische en chemische stoffen.

Hoewel de controle die een periodieke structuur kan uitoefenen niet varieert als de structuur en de frequentie beide geschaald worden, is de emissie van op fotonische kristallen gebaseerde lasers tot op heden beperkt tot een klein aantal frequentiegebieden. Dit wordt veroorzaakt doordat de emissie gebaseerd is op discrete overgangen van gebonden elektronen in typische emitters als quantum dots en andere halfgeleider materialen. Dit in tegenstelling tot emissie van vrije elektronen die in principe elke frequentie kunnen uitzenden bij een overgang tussen twee niveaus binnen de continue verdeling van kinetische energietoestanden. Een zeer interessante coherente lichtbron met unieke eigenschappen zou dus gerealiseerd kunnen worden door het combineren van vrije elektronen als emitter met een groot frequentiebereik met de schalering van fotonische kristallen. 
In dit proefschrift bestuderen wij een dergelijke lichtbron die gebaseerd is op coherente emissie van vrije elektronen in een fotonische kristal, welke door ons de fotonische vrijeelektronenlaser (pFEL) genoemd wordt. We presenteren voor het eerst een uitgebreide en diepgaande studie naar de eigenschappen van een pFEL. Wij beperken ons hierbij tot pFELs die elektronenbundels gebruiken met een lage energie (ongeveer $10 \mathrm{keV}$ ). Hierdoor is het mogelijk om compacte elektronbronnen te gebruiken en een compacte laser te realiseren. De benodigde stappen om tot een experimentele realisatie van een microgolf pFEL te komen worden eveneens beschreven in dit proefschrift.

Om het werkingsprincipe en de eigenschappen van een pFEL te bestuderen gebruiken we de zogenaamde "particle-in-cell" (PIC) methode om een numeriek analyse uit te voeren van een laser die door een enkele elektronenbundel wordt aangedreven. De elektronenbundel heeft een kinetische energie van ongeveer $12.5 \mathrm{keV}$ en een stroom van ongeveer $1 \mathrm{~A}$. Het fotonische kristal bestaat uit een rechthoekig patroon van ronde metalen paaltjes die geplaatst zijn in een rechthoekige metalen golfgeleider. De onderlinge afstand tussen de paaltjes is van de orde van enkele millimeters en heeft als gevolgd dat de verwachte emissie van de laser in het microgolfgebied zal liggen. Voor de onderzochte pFEL hebben we een drempelstroom van ongeveer $140 \mathrm{~mA}$ gevonden. Door de pompstroom te verhogen boven de drempel neemt het uitgangsvermogen lineair toe met de stroom, tot ongeveer $1.5 \mathrm{~kW}$ bij een stroom van $1 \mathrm{~A}$. Hierbij is frequentie van de coherente straling ongeveer $16 \mathrm{GHz}$.

Uit de resultaten die voor deze specifieke pFEL verkregen zijn met behulp van de PIC simulaties komt een simpele beschrijving van de werking van de pFEL naar voren. De werking van de laser is gebaseerd op het principe van constructieve interferentie van een groot aantal korte golfpakketjes die uitgezonden worden door het fotonisch kristal als reactie op de passerende elektronen. De emissie van elk van deze golfpakketjes kan beschouwd worden als spontane emissie van Čerenkov straling door elk elektron in het fotonisch kristal. De Čerenkov straling kan veranderen in gestimuleerde emissie door terugkoppeling van de spontane emissie op de elektronen. Hiervoor moet gelden dat het fotonisch kristal een elektromagnetisch veld ondersteunt met een longitudinale elektrische veldcomponent en dat deze een fasesnelheid bevat die gelijk is aan de snelheid van de elektronen. De terugkoppeling resulteert in het opeenhopen van de elektronen vanuit de oorspronkelijke continue verdeling in de bundel. Tijdens dit proces worden gemiddeld mee elektronen vertraagd dan versneld. De kinetische energie die hierdoor beschikbaar komt wordt op een coherente wijze toegevoegd aan een groeiende elektromagnetische golf. Door de snelheid van de elektronen te variëren is ook een andere fasesnelheid van het elektromagnetisch veld nodig wat tot een verandering van de frequentie van de laser leidt.

Door gebruik te maken van PIC simulaties is het mogelijk gedetailleerde informatie 
over het functioneren van een pFEL te verkrijgen. Echter, zo'n gedetailleerde beschrijving is niet altijd noodzakelijk en handig, vooral omdat de PIC berekeningen tijdsintensief zijn. Het is daarom wenselijk om een alternatieve methode te vinden die gebruikt kan worden om te testen of een bepaald fotonisch kristal geschikt is voor gebruik in een pFEL. Een snelle methode heeft als belangrijk voordeel dat het mogelijk wordt een grote verscheidenheid aan fotonische kristallen met elkaar te vergelijken, of een groot aantal verschillende pompparameters te onderzoeken, bijvoorbeeld als voorbereiding op een experimentele demonstratie. Een dergelijke methode is gevonden in de vorm van een lineair model dat vervolgens gevalideerd wordt met behulp van PIC simulaties. Dit lineair model kan onder meer gebruikt worden om de drempel van de laser en de kleinsignaalversterking te voorspellen.

Het hoge vermogen dat een pFEL gepompt door een enkele elektronenbundel kan produceren is veelbelovend voor verschillende toepassingen. Echter, de grootste potentie van de laser ligt in het schalen van de frequentie naar verschillende spectrale gebieden. Als de spatiële periode van het kristal verkleind wordt met een bepaalde factor, dan zal de laser frequentie met eenzelfde factor verhoogt worden. Echter het vermogen van de laser zal alleen dan constant blijven als ook alle andere parameters gelijk blijven, in het bijzonder geldt dit voor de energie en de stroom van de elektronenbundel die de laser pompt. $\mathrm{Nu}$ zal door het schalen het oppervlak dat beschikbaar is voor de elektronenbundel afnemen, zodat de stroom alleen gelijk kan blijven door de stroomdichtheid toe te laten nemen. De stroomdichtheid is echter fundamenteel gelimiteerd door de Coulomb repulsie tussen de elektronen. Bij het schalen naar hogere frequenties zal de stroom in de enkele bundel onvermijdelijk afnemen en daarmee ook het vermogen van de laser.

Gelukkig biedt een fotonisch kristal een alternatieve aanpak voor het schalen van de frequentie waarbij het vermogen van de laser gelijk kan blijven. Fotonische kristallen bieden van nature vele parallelle vacuümkanalen. De afname van de stroom in een enkele bundel kan dus gecompenseerd worden door meer bundels door het kristal te sturen zodat de totale bundelstroom gelijk blijft. Het uitgangsvermogen van de laser kan zo in stand gehouden worden. Een bijkomend risico is echter dat het grotere pompvolume kan leiden tot het oscillatie in meerdere transversale modi. Hierdoor kan de helderheid van de bron afnemen.

Om deze methode van schalen te onderzoeken, vergroten we de transversale afmeting van het kristal, bij gelijkblijvende periodiciteit, zodat de laser gepompt kan worden met maximaal zeven elektronenbundels. Het uitgangsvermogen neemt toe met het aantal pompbundels en bedraagt ongeveer $8 \mathrm{~kW}$ bij zeven elektronenbundels. Maar belangrijker is dat de output van de laser bijna volledig in een enkele transversale veldverdeling wordt 
gegenereerd, meer dan 95\% van het totale vermogen bevindt zich in de laagste orde transversale veldverdeling. De verklaring dat slechts één van de vele veldverdelingen dominant is wordt gezocht in het feit dat de elektronen mono-energetisch zijn. Hierdoor gedragen de elektronen zich als een homogeen verbreed versterkingsmedium en zullen de verschillende transversale veldverdelingen in competitie strijden om de versterking.

Deze resultaten maken het volgens mogelijk dat een toekomstige pFEL gepompt kan worden door vele, d.w.z. honderden tot zelfs duizenden, pompbundels, bijvoorbeeld geproduceerd door zogenaamde veld-emissie array elektronenbron. Het grote aantal bundels staat een voldoende lage stroom per bundel toe terwijl de totale stroom voldoende hoog is om in een pFEL een vermogen van enkele Watts te produceren in een enkele transversale veldverdeling en op een frequentie in het $\mathrm{THz}$ gebied.

In het tweede deel van dit proefschrift wordt een experimentele demonstratie van het pFEL concept voorbereid. De versterking in een pFEL draait om het fotonisch kristal. Het kristal dient een elektromagnetisch veld met een longitudinaal elektrische veldcomponent te ondersteunen, immers het is deze veldcomponent die verantwoordelijk is voor de versterking. Een experimentele verificatie van deze veldcomponent is belangrijk voor het karakteriseren van de fotonische kristallen die in een pFEL geplaatst kunnen worden. Een groot probleem is dat tot op heden geen methode bekend was waarmee individuele componenten van het elektromagnetische veld in het inwendige van een fotonisch kristal gemeten kunnen worden.

We laten een methode zien die geschikt is voor het in kaart brengen van de elektromagnetische velden in het inwendige van een fotonisch kristal. De methode berust op het meten van de verschuiving in de resonantiefrequenties wanneer het fotonische kristal in een resonator wordt geplaatst en het veld in het fotonische kristal verstoord wordt door een verstrooier die kleiner is dan de golflengte. In onze experimenten is een bolvormige verstrooier gebruikt om het dominante longitudinale elektrische veld in een specifiek fotonisch kristal te meten. We vinden een goede overeenkomst tussen gemeten en berekende longitudinale elektrische veldsterktes zonder gebruik te maken van fit parameters in het model.

Uit onze analyse is duidelijk gebleken dat de pFEL een veelbelovend concept is voor het maken van verstembare, coherente Čerenkov straling met een aanzienlijk vermogen. Voor het demonstreren en onderzoeken van dergelijke lasers, wordt in dit proefschrift ook een experimenteel ontwerp gepresenteerd dat straling kan generen in het microgolfbereik. Een ontwerp vereist de combinatie van een viertal verschillende technologiën, namelijk elektronenbundelgeneratie en -transport, hoogspannings-, microgolf- en vacuümtechnologie. Als elektronenbron is gekozen voor een standaard commerciële elektronenbron welke 
gebruikt wordt in lopende-golfbuizen. Deze bron levert een bundelstroom van ongeveer $2 \mathrm{~A}$ bij een nominale bundelenergie van $14.2 \mathrm{keV}$. Om de verstemming van de pFEL te onderzoeken, kan de bundelenergie gevarieerd worden van $10 \mathrm{keV}$ tot $15 \mathrm{keV}$. De benodigde hoogspanningsvoedingen zijn ontworpen en gerealiseerd. Daarnaast presenteren we het ontwerp en de realisatie van de elektromagneet die nodig is om de elektronenbundel door het fotonisch kristal te leiden. Het ontwerp is gebaseerd op de numerieke optimalisatie van de elektronen banen uitgaande van een realistisch model voor zowel de elektronbron als magneet. Tenslotte hebben we een ontwerp en prototype gemaakt voor het fotonische kristal. In het ontwerp van de pFEL is ruimte voor meer dan honderd eenheidscellen van het fotonisch kristal. Bij het maximale aantal eenheidscellen is de verwachte drempel van de laser voor de pompstroom ongeveer $7 \mathrm{~mA}$. We verwachten, nadat de complete opstelling is samengebouwd, dat de eerste systematische experimentele analyse van een pFEL mogelijk wordt.

Het theoretische model dat in dit proefschrift wordt gepresenteerd, en het bijbehorende voorstel voor een demonstratie van de pFEL, kan een sleutelrol spelen in het ontstaan van een nieuwe groep van compacte, hoogvermogen laserbronnen in het microgolf- en $\mathrm{THz}$ frequentiebereik. 



\section{List of publications}

\section{Journal publications}

- T. Denis, M. W. van Dijk, J. H. H. Lee, P. J. M. van der Slot and K.-J. Boller, High brightness power up-scaling in photonic free-electron lasers by using multiple electron beams, in preparation

- T. Denis, M. W. van Dijk, J. H. H. Lee, P. J. M. van der Slot and K.-J. Boller, Photonic free-electron lasers: frequency tunable photonic crystal laser, in preparation

- T. Denis, B. Reijnders, J. H. H. Lee, P. J. M. van der Slot, W. L. Vos and K.-J. Boller, Method to map individual electromagnetic field components inside a photonic crystal Opt. Express 20, pp. 22902-22913 (2012).

- P. J. M. van der Slot, T. Denis, J. H. H. Lee, M. W. van Dijk and K.-J. Boller, Photonic free-electron lasers, IEEE Photon. J. 4, pp. 570-573 (2012).

\section{Conference Proceedings}

- J. H. H. Lee, M. W. van Dijk, T. Denis, P. J. M. van der Slot and K.-J. Boller, Dynamics of a multi-beam photonic free-electron laser, in: Proceedings of FEL2012, to be published (2012).

- T. Denis, M. W. van Dijk, J. H. H. Lee, P. J. M. van der Slot and K.-J. Boller, A photonic free-electron laser, in CLEO: QELS-Fundamental Science, paper QM2E.8 (2012).

- J. H. H. Lee, M. W. van Dijk, T. Denis, P. J. M. van der Slot and K.-J. Boller, Power scalability of a low-gain photonic free-electron laser, in: Thirteenth International IEEE Vacuum Electronics Conference, pp. 219-220 (2012). 
- J. H. H. Lee, M. W. van Dijk, T. Denis, P. J. M. van der Slot and K.-J. Boller, Study of beam focusing techniques for a power- and frequency scalable photonic freeelectron laser, in: Thirteenth International IEEE Vacuum Electronics Conference, pp. 221-222 (2012).

- T. Denis, M. W. van Dijk, J. H. H. Lee, P. J. M. van der Slot and K.-J. Boller, Singlemode power scaling in a multi-beam photonic free-electron laser, in: Thirteenth International IEEE Vacuum Electronics Conference, pp. 223-224 (2012).

- J. H. H. Lee, T. Denis, P. J. M. van der Slot, K.-J. Boller, The design of a multibeam electron gun for a photonic free-electron laser, in: Proceedings of FEL2011, pp. $427-430(2011)$

- T. Denis, B. Reijnders, J. H. H. Lee, P. J. M. van der Slot and K.-J. Boller, Proof of principle: the single beam photonic free-electron laser, in: Proceedings of FEL2010, pp. 644-647 (2010).

- T. Denis, P. J. M. van der Slot and K.-J. Boller, Slow-wave structure for a photonic free-electron laser, in: Eleventh International IEEE Vacuum Electronics Conference, pp. 389-390 (2010).

- T. Denis, P. J. M. van der Slot and K.-J. Boller, A single beam photonic freeelectron laser, in: Proceedings of the 2009 Annual Symposium of the IEEE Photonics Benelux Chapter, pp. 93-96 (2009).

- T. Denis, P. J. M. van der Slot and K.-J. Boller, Experimental design of a single beam photonic free-electron laser, in: Proceedings of FEL2009, pp. 431-434 (2009).

- P. J. M. van der Slot, T. Denis, K.-J. Boller, The photonic FEL: Toward a handheld THz FEL, in: Proceedings of FEL2008 pp. 231-234 (2008).

\section{Oral presentations}

- T. Denis, M. W. van Dijk, J. H. H. Lee, P. J. M. van der Slot and K.-J. Boller, Working principle of photonic free-electron lasers, MESA+ Annual Meeting, Enschede, NL (2012).

- K.-J. Boller, J. H. H. Lee, T. Denis and P. J. M. van der Slot, Photonic free-electron lasers - coherent Cerenkov radiation from photonic crystals, Physical Colloquium Universitee de Rennes (invited), Rennes, FR (2012). 
- T. Denis, M. W. van Dijk, J. H. H. Lee, P. J. M. van der Slot and K.-J. Boller, Fundamental physics of a photonic free-electron laser, 7th CST European User Conference, Mannheim, DE (2012).

- J. H. H. Lee, M. W. van Dijk, T. Denis, P. J. M. van der Slot and K.-J. Boller, Power scalability of a multi-beam photonic free-electron laser, 7th European User Conference, Mannheim, DE (2012).

- T. Denis, M. W. van Dijk, J. H. H. Lee, P. J. M. van der Slot and K.-J. Boller, A photonic free-electron laser, CLEO:QELS, San Jose, USA (2012).

- T. Denis, M. W. van Dijk, J. H. H. Lee, P. J. M. van der Slot and K.-J. Boller, Tunable coherent Cerenkov radiation from photonic crystals, Group colloquium Optical Solid State Physics (invited), Groningen, NL (2012).

- M. W. van Dijk, T. Denis, J. H. H. Lee, P. J. M. van der Slot and K.-J. Boller, The photonic free-electron laser: coherent Cerenkov radiation from photonic crystals, 24st NNV-symposium on Plasma Physics and Radiation Technology, Lunteren, NL (2012).

- T. Denis, B. Reijnders, J. H. H. Lee, P. J. M. van der Slot and K.-J. Boller, Mapping electromagnetic fields inside photonic crystals, 35th Annual Meeting NNV AMO Lunteren, Lunteren, NL (2011).

\section{Poster presentations}

- T. Denis, M. W. van Dijk, J. H. H. Lee, P. J. M. van der Slot and K.-J. Boller, Relaxation oscillation of the wave front tilt in a photonic free-electron laser, 36th Annual Meeting NNV AMO Lunteren, Lunteren, NL (2012).

- T. Denis, M. W. van Dijk, J. H. H. Lee, P. J. M. van der Slot and K.-J. Boller, Linear gain and gain saturation in a photonic free-electron laser, 34th International Free-Electron Laser Conference, Nara, Japan (2012).

- J. H. H. Lee, M. W. van Dijk, T. Denis, P. J. M. van der Slot and K.-J. Boller, Dynamics of a multi-beam photonic free-electron laser, 34th International Free-Electron Laser Conference, Nara, Japan (2012).

- T. Denis, M. W. van Dijk, J. H. H. Lee, P. J. M. van der Slot and K.-J. Boller, Single-mode power scaling in a multi-beam photonic free-electron laser, Thirteenth International IEEE Vacuum Electronics Conference, Monterey, USA (2012). 
- J. H. H. Lee, M. W. van Dijk, T. Denis, P. J. M. van der Slot and K.-J. Boller, Power scalability of a low-gain photonic free-electron laser, Thirteenth International IEEE Vacuum Electronics Conference, Monterey, USA (2012).

- J. H. H. Lee, M. W. van Dijk, T. Denis, P. J. M. van der Slot and K.-J. Boller, Study of beam focusing techniques for a power- and frequency scalable photonic free-electron laser, Thirteenth International IEEE Vacuum Electronics Conference, Monterey, USA (2012).

- T. Denis, M. W. van Dijk, J. H. H. Lee, P. J. M. van der Slot and K.-J. Boller, Small signal gain calculations for a photonic free-electron laser, Physics@FOM, Veldhoven, NL (2012).

- T. Denis, M. W. van Dijk, J. H. H. Lee, P. J. M. van der Slot and K.-J. Boller, Pierce gain calculations for a photonic free-electron laser, MESA+ Annual Meeting, Enschede, NL (2011).

- J. H. H. Lee, T. Denis, P. J. M. van der Slot, K.-J. Boller, The design of a multibeam electron gun for a photonic free-electron laser, 33rd International Free-Electron Laser Conference, Shanghai, China (2011).

- T. Denis, B. Reijnders, J. H. H. Lee, P. J. M. van der Slot and K.-J. Boller, High power microwave photonic free-electron laser, Physics@FOM, Veldhoven, NL (2011).

- T. Denis, B. Reijnders, J. H. H. Lee, P. J. M. van der Slot and K.-J. Boller, Proof of principle: the single beam photonic free-electron laser, 32nd International FreeElectron Laser Conference, Malmö, SE (2010).

- T. Denis, P. J. M. van der Slot and K.-J. Boller, The compact photonic FEL - a proof of principle, 66th Scottish Universities Summer School in Physics, Edinburgh, UK (2010).

- T. Denis, P. J. M. van der Slot and K.-J. Boller, Slow-wave structure for a photonic free-electron laser, Eleventh International IEEE Vacuum Electronics Conference, Monterey, USA (2010).

- T. Denis, P. J. M. van der Slot and K.-J. Boller, A single beam photonic free-electron laser, Annual Symposium of the IEEE Photonics Benelux Chapter, Brussels, BE (2009). 
- T. Denis, P. J. M. van der Slot and K.-J. Boller, Interaction structures for a photonic free-electron laser, 31st International Free-Electron Laser Conference, Liverpool, UK (2009).

- T. Denis, P. J. M. van der Slot and K.-J. Boller, Experimental design of a single beam photonic free-electron laser, 31st International-Free Electron Laser Conference, Liverpool, UK (2009).

- T. Denis, P.J.M. van der Slot and K.-J. Boller, The photonic free-electron laser, Physics@FOM, Veldhoven, NL (2009).

- T. Denis, P.J.M. van der Slot and K.-J. Boller, Photonic Crystals for a handheld THz Source: the photonic free-electron laser, Nanometa Conference, Seefeld in Tirol, AT (2009).

- P. J. M. van der Slot, T. Denis, K.-J. Boller, The photonic FEL: toward a handheld THz FEL, 30th International Free-Electron Laser Conference, Gyeongju, KR (2008). 



\section{Acknowledgments}

It is a fine tradition that a $\mathrm{PhD}$ thesis ends with the acknowledgments. Malicious tongues might say this is to make sure that something of the thesis is actually read. However, in my opinion that is absolute nonsense because such statement undermines the value of all the support and help I experienced during the time of my PhD. As many of you know, I had a number of personal and work-related throwbacks, therefore I certainly owe many kind and helpful people a big thank you.

First of all I want to thank Klaus Boller, who gave me the opportunity to work in the Laser Physics and Nonlinear Optics (LPNO) group. I have experienced Klaus as a broadly interested researcher, with an open mind to any idea, which is what I appreciate most of his research approach. Further, in countless hours I could enjoy discussions with him about physics in general and laser physics in particular. In these discussions Peter van der Slot was also often involved, especially, when a well-educated advice on the specific physics of an FEL was needed. What I am most thankful for is Peter's and Klaus' support during the writing process of my thesis. My tight writing-schedule put a high-work load on both of them during the last weeks. Thank you for tolerating this and working that hard for me!

The next people I want to thank are all of my colleagues during the years in the Laser Physics and Nonlinear Optics group. Over the years a lot of people come and go, all of them had an impact to this thesis. Thank you: Ab, Arie, Arsen, Bert, Bob, Bas-Jan, Carin, Chris, Fred B., Fred G., Gerard, Jean, Joan, Johan, Jonathon, Jörn, Jelle, Jurjen, Kasper, Klaus, Lantian, Leon, Matthijs, Marc, Mark, Martijn, Muharrem, Peter, Piet, Rob, Robert, Rolf, Ruud, Simone, Wiebke, Willem, Yin. However, I should not miss the opportunity to highlight the work of Bob, Marc and Gerard. Bob and Marc, it was a great pleasure to work with you, seeing both of you picking up the advanced topic of a pFEL in a short time and contributing to so many publications. Meanwhile both of you did never get annoyed with my straightforward approach of supervision at all, which is an achievement on its own ;-). Gerard, I owe you a big thank you for the CAD design of 
the laser and helping me to assemble it.

No group ever works isolated from its surrounding environment. Thus, I also want to thank our old and new neighbors. In the old building we shared the office floor with the company NCLR. Thanks for the great conversations during coffee breaks and social activities: Arco, Gerald, Hein, Leon, Liviu, Ramon, but most importantly Jacob, who helped me to get the hang of low and high-voltage electronics while he worked at NCLR and even afterwards! After the movement our neighbor is the group Complex Photonic Systems and we share the coffee table with them. In addition, I also share a similar research topic with them. So thank you to all of the COPS members, for the advice, the magnificent coffee (Oh YEAH), and the occasional but essential cake. Further, I want especially thank Willem Vos, the chair of COPS, for encouraging me to write my first paper and also showing me how to sell it as a Rolls Royce and push it forward in the community, such that it is noticed. It took me some time to get there, but I am proud on the paper we published together and hope there are more to come in the future.

While not every optics group in the university is neighbor in space, but all of them are a neighbor in science. Since recently, the SRO Applied Nanophotonics led by Pepijn Pinske makes sure to bring all these scientists on a regular basis together. This allowed me to have countless useful discussion with my fellow optics colleagues at the University, which I really appreciate. I also want to acknowledge the help from outside the University. Thank you Monika Balk, Mike Clark and Christopher Gilmour. Mike and Chris both work at the company TMD Technologies in the UK, which provided essential components and invaluable technological know-how to the project. I met Monika first at a conference where she introduced met to the particle-in-cell code of CST, which became very important to my work. But certainly more important was the fact, that for urgent requests I could always approach her in person. Despite her busy schedule I would always receive a precise and good answer to my specific problem in a very short time. I my opinion that is more than usual customer service!

Next to work, also PhD students have a live and that is very much needed to make sure that you keep having fresh and new ideas. Sometimes it is also just necessary in order to forget all the things that go wrong at work. I forgot such bad experiences by doing sports. I went climbing with Laura, hit the gym with Silja, and most importantly played Ultimate Frisbee. While I was for some time rather unlucky with my fitness, I nevertheless enjoyed a lot of happy hours with the members of the Disc Devils Twente, either at a tournament, at training, on a competition day or at the countless social activities. Thank you to all of you. Ultimate rules!

Neben Frisbee hab ich den Doktorstress mit Blasmusik vertrieben. Es ist immer wieder 
erfrischend nach Nienborg zu kommen, sich das Horn zu packen und Musik zu machen. Dabei vergisst man spielend all die Dinge die mal wieder schief gegangen sind im Labor. Danke für all die schönen Stunden an alle Mitglieder der Comboband und alle Mitglieder des Musikverein Nienborg.

I also want to thank all of my friends I met at school, university or elsewhere. Danke an die "Jungs": Amjürn, Michi, Möthe, Öt, Onkel U., Pflipse, Sebi und Robert. Julia danke für ein offenes Ohr und das gesündestes Essen aller Zeiten. Silja, we did many nice things together and I will remember these and no else. Jan thank you for introducing me to the second use of a Dutch work permit and the great fun that results from that. Rudi and Jürgen there is no such thing I could not discuss with you, that is invaluable. Gunnar I will not forget our meeting at the CLEO, it was great fun. Ijsbrandt, Judith and Hinderik I most likely will never know all the famous Dutch stars, but Pub Quiz was always fun and I got to know the high-quality Dutch songs, such as: "Hey Marlous". Ruud and Thomas thanks for the graphical design advices. Linda, it was really nice that you let me move in when I needed a place to live! Donna, I am sorry for "such as", comma massacre, chaotic word order, and the ability of Germans for placing the verb at the very, very, very end of a sentence. Thanks for not saying "bugger off", but offering me a tea and proof-reading my thesis.

Der letzte Abschnitt dieser Danksagung gebührt meiner Familie. Obwohl wahrscheinlich weder mein Bruder, meine Mama oder mein Papa, viel von dem verstehen was in dieser Arbeit untersucht wurde, sind Sie trotz allem der Schlüssel dazu das ich einen Doktor machen konnte. Ohne Euch wäre ich heute nicht hier. Danke für die Unterstützung während meiner Schulzeit und meines Studiums. Danke das Ihr immer für mich da seid wenn es nötig ist und das trotzdem ich manchmal so richtig stuppig sein kann! 



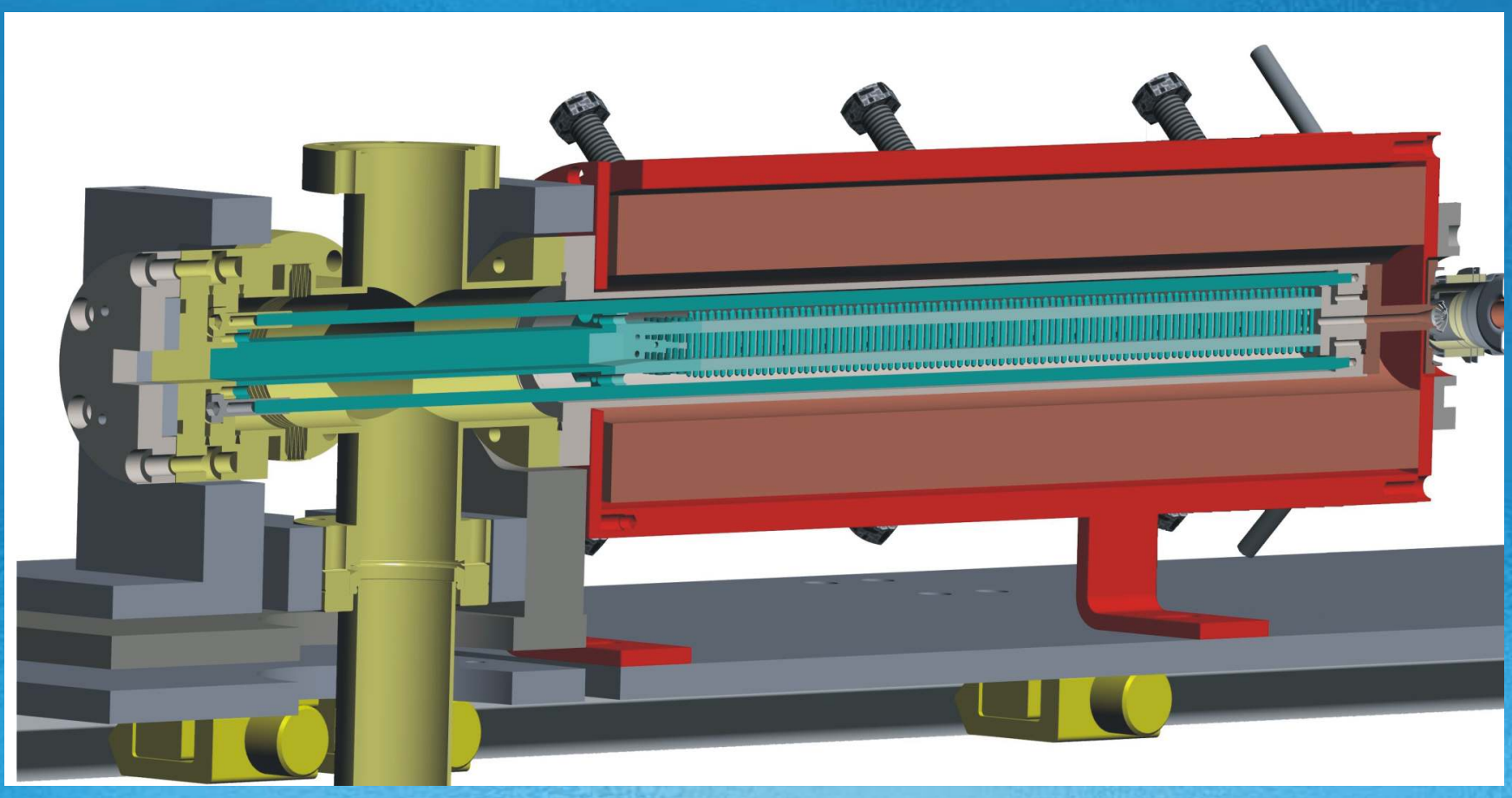

Photonic free-electron lasers (PFDL) transform part of the kinetic energy from electrons into a coherent electromagnetic wave. In a pFDL one or multiple electron beams travel through a photonic crystal. The photonic crystal reduces the phase velocity of the electromagnetic wave such that, for a certain frequency, the electromagnetic wave and the electrons are velocity matched. In this case, electromagnetic waves with a non-zero electric field along the propagation direction of the electrons can induce a bunching in the beam. This leads to stimulated emission. If the stimulated emission is sufficiently strong, the electromagnetic field is amplified exponentially until saturation. In this thesis the theory and design of pFDLs at microwave frequencies are described. 